Universidade de SÃo Paulo

Instituto de Física de SÃo CARLos

\title{
FUNÇÕES DE ONDA PARA O ÁTOMO DE LÍTIO NO FORMALISMO HIPER- ESFÉRICO.
}

José Paulo D'Incao

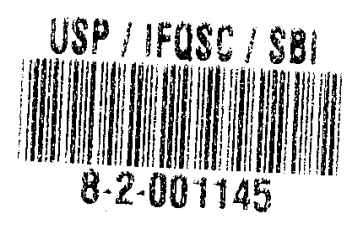

Dissertação apresentada ao Instituto de Física de São Carlos, da Universidade de São Paulo, para obtenção do título de Mestre em Ciñmni ฉc: Física Básica.

Orientador: Prof. Dr. José Eduardo Martinho Hornos

Departamento de Física e Ciência dos Materiais

SÃo CARLOS - 1997 
Funções de onda para o átomo de lítio em coordenadas hiperesféricas / José Paulo D'Incao.-São Carlos, 1997.

$<139>\mathrm{p}$.

Dissertação (Mestrado)-Instituto de Física de São Carlos, 1997.

Orientador: Prof. Dr. José Eduardo Martinho Hornos 1. Formalismo Hiperesférico. 2. Representação mista. I. Título. 
MEMBROS DA COMISSÃO JULGADORA DA DISSERTAC̣̃̃O DE MESTRADO DE JOSÉ PAULO D'INCAO APRESENTADA AO INSTITUTO DE FÍSICA DE SÃO CARLOS, DA UNIVERSIDADE DE SÃO PAULO, EM 25 DE MARÇO DE 1997.

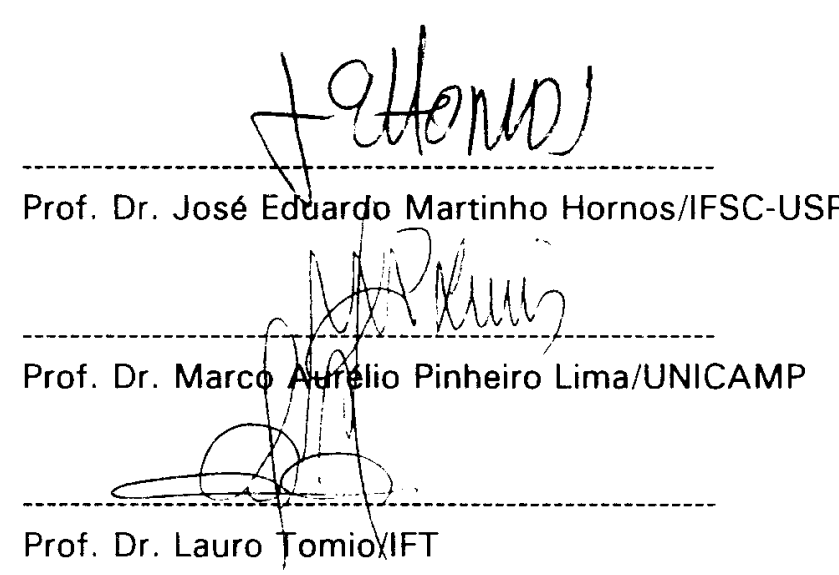


Aos meus pais

Denise e José Arthur. 
Este trabalho teve o apoio financeiro da FAPESP. 


\section{Agradecimentos}

- Gostaria de agradecer primeiramente ao prof. Hornos, pela dedicação e seriedade no decorrer do desenvolvimento deste projeto, e pela contribuição na minha formação científica e profissional

- Agradeço pessoal do grupo e a todos os colegas do departamento por toda a ajuda. em especial ao Mauru. Ghandi e Jean pelo auxílio pessoal na caçada às curvas de potencial

- ans amigos Marconi, Cláudia e Nescau, pelo companheirismo, sinceridade e confiança

- aos colegas de sala Marconi e Gabriel, e os vizinhos Cintia, Ana Carolina, Ligia, Marletta, Fernandinho e Marcelo, pela paciência

- aos amigos de sempre Cláudia(Pio), Jabáh. Esponja e Fernandão

Enfim. à Sandra, pela a amizade, pela presença e tudo o que a faz especial. 


\section{Índice}

1 Introdução

2 Formalismo Hiperesférico para o Átomo de Lítio

2.1 Termo Cinético. Hiperesféricos Harmônicos _. . . . . . . . . . 25

2.2 As interações Coulombianas . . . . . . . . . . . . . . . . . 30

2.3 Curvas de Potencial . . . . . . . . . . . . . . . . . 33

3 Grupos Finitos

3.1 Definições. Exemplos . . . . . . . . . . . . . . . . . 37

3.1.1 Sub-grupos. Homomorfismo e Isomorfismo . . . . . . . . . . . 43

3.1 .2 Teorema de Lagrange . . . . . . . . . . . . . . . . . 45

3.1.3 Classes Conjugadas $\ldots \ldots \ldots \ldots$. . . . . . . . . 46

3.1.4 Sub-grupos Invariantes. Grupo Quociente . . . . . . . . . . . 49

3.2 Representações . . . . . . . . . . . . . . . . . . . . 50 50

3.2.1 Representações Equivalentes e Caracter . . . . . . . . . . . . . . 51

3.2.2 Redutibilidade e Irredutibilidade $\ldots \ldots \ldots$. . . . . . . . 52

3.2.3 Representações Unitárias . . . . . . . . . . . . . . . . . . . 54

4 Grupos de Permutação

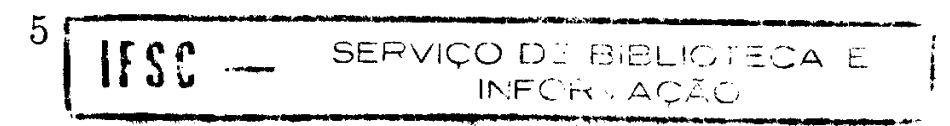


4.1 Diagramas de Young. Young Tableaux. Irreps . . . . . . . . . . . 55

4.2 Young Tableau. Dimensão das Irreps _ . . . . . . . . . . . . . 56

4.3 Lei de Ramificação . . . . . . . . . . . . . . . . . . . . . . . . . . 58

4.4 Atuação do $\mathbf{S}_{n}$ em Funções de $n$ Variáveis . . . . . . . . . . . . . . 59

4.5 Álgebra do Grupo. Operadores de Young . . . . . . . . . . . . . . . 61

4.5.1 Representação Regular . . . . . . . . . . . . . . . . . 62

4.5 .2 Álgebra do Grupo . . . . . . . . . . . . . . . . . . . 63

4.5 .3 Operadores de Young . . . . . . . . . . . . . . . 65

4.6 Operadores de Projeção $\quad \ldots \ldots$. . . . . . . . . . . . . . . . 70

4.6 .1 Definição . . . . . . . . . . . . . . . 70

$4.6 .2 \quad$ Bases Irredutíveis para o $\mathbf{S}_{3} \ldots \ldots \ldots \ldots \ldots$

4.6.3 Operadores de Projeção e Álgebra do Grupo . . . . . . . . . . . 75

4.7 Produto de Kronecker de Irreps _. . . . . . . . . . . . . . . . 78

4.7.1 Séries de Clebsch-Gordan . . . . . . . . . . . . 78

4.7 .2 Coeficientes de Clebsch-Gordan . . . . . . . . . . . 81

5 Simetrização das Funções de Canal $\quad 85$

5.1 Produto de Kronecker : Spin $\otimes$ Angular . . . . . . . . . . . . . . 85

5.2 Simetria Mista da Função de Spin . . . . . . . . . . . . . . . . 87

5.3 Simetria Mista da Função Angular . . . . . . . . . . . . . . . 88

6 Anti-Simetria da Função de Onda Total 93

7 Equação Radial

7.1 Cálculo das Curvas de Potencial . . . . . . . . . . . . . 96

7.2 Equação Radial. Acoplamentos $P_{\mu \nu}$ e $Q_{\mu \nu} \ldots \ldots \ldots . . \ldots . . . . . .98$ 
8 Resultados e Conclusões

8.1 Funções Simetrizadas para $L=0 \ldots \ldots \ldots$. . . . . . . . . 102

8.2 Curvas de Potencial . . . . . . . . . . . . . . . . . . . 103

8.3 Energia Adiabática Extrema, $E_{E A A} \ldots \ldots \ldots . \ldots . \ldots$

8.4 Conclusões e Perspectivas . . . . . . . . . . . . . . . . . . . 112

$\begin{array}{ll}\text { A Curvas de Potencial } \lambda_{9}=2 & 115\end{array}$

$\begin{array}{lll}\text { B Atuação de } \rho_{11}^{(m)} \text { em } \lambda_{9}=2,4,6 \text { e } 8 & 118\end{array}$

C Bases para as Funções de Canal, Resultantes do Projetor $\rho_{11}^{(m)} \quad 133$ 


\section{Lista de Tabelas}

2.1 Degenerescéncia das Curvas de Potencial $L=0$, em $R=0 . \quad \ldots . . \quad 35$

3.1 Tabela de multiplicação do grupo $\mathbf{C}_{\mathbf{3 v}} \ldots \ldots \ldots$. . . . . . . . . . . . 38

3.2 Tabela de multiplicação do grupo cíclico de ordem 4. . . . . . . . . . . 39

3.3 Tabela de multiplicação do grupo four. . . . . . . . . . . . . . . . . . . 39

3.4 Tabela de multiplicação do grupo de Permutação $\mathbf{S}_{3} \ldots \ldots \ldots$. . . . . . . . 41

3.5 Tabela de multiplicação do grupo $\mathcal{A}_{3} \ldots \ldots \ldots$. . . . . . . . . . . . 42

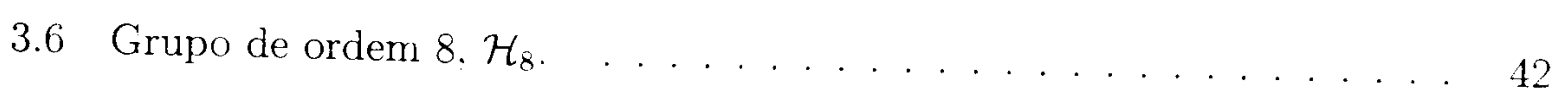

4.1 Caracters para os grupos $\mathbf{S}_{\mathbf{3}}, \mathbf{S}_{\mathbf{4}}$ e $\mathbf{S}_{5} \ldots \ldots \ldots \ldots$

4.2 Séries de Clebsch-Gordan para o grupo $\mathbf{S}_{\mathbf{3}} \ldots \ldots \ldots$. . . . . . . . 80

4.3 Séries de Clebsch-Gordan para o grupo $\mathbf{S}_{\mathbf{4}} \ldots \ldots \ldots \ldots$. . . . . . . 80

4.4 Séries de Clebsch-Gordan para o grupo $\mathbf{S}_{\mathbf{5}} \ldots \ldots \ldots \ldots$

4.5 Coeficientes de Clebsch-Gordan : [21] $\times[21]=[3]+[21]+\left[1^{3}\right] . \ldots 2$

4.6 Coeficientes de Clebsch-Gordan : $[31] \times[31]=[4]+[31]+\left[2^{2}\right]+\left[21^{2}\right] . \quad 82$

4.7 Coeficientes de Clebsch-Gordan : $[31] \times\left[2^{2}\right]=[31]+\left[21^{2}\right] \ldots . . . . \quad 82$

4.8 Coeficientes de Clebsch-Gordan : $[41] \times[41]=[5]+[41]+[32]+\left[31^{2}\right] . \quad 83$

8.1 Limensäo dos setores $\lambda_{4}=2.4 .6 \mathrm{co}$. para $L=0$. 
8.2 Número de funções de canal para o átomo de lítio para $L=0$ e $S=1 / 2$, até $\lambda_{9}=28$.

8.3 Energia do estado fundamental para o átomo de lítio na aproximação adiabática extrema 


\section{Lista de Figuras}

4.1 O espaço do Grupo. . . . . . . . . . . . . . . . . . . 63

4.2 Álgebra dos Operadores de Projeção do Grupo $\mathbf{S}_{3} \ldots \ldots \ldots \ldots$

8.1 Curvas de Potencial $-U_{\mu}(R)$ e $-U_{\mu}(R) / 2 R^{2}$ para o átomo de lítio, na configuração $(0.0 .0)$ com $\lambda_{9}$ até $28 \ldots \ldots \ldots \ldots \ldots$

8.2 Curvas de Potencial $-U_{\mu}(R)$ e $-U_{\mu}(R) / 2 R^{2}$ para o átomo de lítio com $\lambda_{9}$ até 28 , adicionando as funções de momento angular orbital arbitrário. 106

8.3 Curvas de potencial na região dos anti-crossings, figura 8.2.(1) . . . 107

8.4 Curvas de potencial na região dos anti-crossings, figura 8.2.(2) . . . 108

8.5 Convergência das curvas de potencial de acordo com o número de canais.

De cima para baixo temos curvas com 2, 7, 18, 42, 44, 46, 49, 52, 55, 59,

$63,67,72$ e 77 canais.. . . . . . . . . . . . . 110

8.6 Função hiperesférica radial para o estado fundamental do átomo de lítio,

a partir da curva de potencial mais baixa com 77 canais. . . . . . . 112

8.7 A curva de potencial mais baixa. . . . . . . . . . . . . . 114

A.1 Curvas de Potencial para a aproximação $\lambda_{9}=2 \ldots \ldots \ldots \ldots$. . . . 117 


\section{Resumo}

O problema quântico não relativístico de quatro corpos tem recebido nos últimos anos uma atenção muito especial. O desenvolvimento de técnicas matemáticas e computacionais tornou viável a obtenção de resultados para a equação de Schrödinger. Essa classe de problemas inclui o átomo de lítio, que é desenvolvido neste projeto sob o formalismo hiperesférico adiabático (HAA), por ser um sistema altamente correlacionado. O ground state e suas excitações tem spin total $S=1 / 2$, portando a representação mista [21], do grupo de permutação $S_{3}$. Nós usamos como base para as funções de canal os hiperesféricos harmonicos do grupo de rotação $S O(9)$ onde nós impusemos a representação mista [21] do $S_{3}$, através do uso dos operadores de projeção para esta simetria, de modo que a composição spin®espacial seja uma representação totalmente anti-simétrica. As curvas de potencial são calculadas. nesta base, para uma configuração de momento angular $\left(l_{1}, l_{2}, l_{3}\right)$, e posteriormente adicionamos outras funções, na configuração $(0,0,0)$, para melhorarmos a convergência da curva na região de $R$ pequeno. Neste trabalho nós tratamos a equação hiperesférica radial dentro da aprximação adiabática extrema (EAA), sem as correções não adiabáticas $P_{\mu \nu}(R)$ e $Q_{\mu \nu}(R)$, obtendo um primeiro resultado para a energia do estado fundamental $E_{E A A}^{(0)}$, preparando procedimento para futuras melhoras na precisão e no cálculo de um novo conjunto de funções de base. 


\begin{abstract}
The quantum non-relativistic four-body problem have received in the last years a very special attention. The development of new mathematic and computation techniques, make viable the acquisition of $a b$ initio solutions for the Schrödinger equation. This class of problem include the lithium atom, who is treat here within hyperspherical adiabatic approach (HAA). due the high eletronic correlation energy. The ground-state and their excited states have total spin $S=1 / 2$, carrying the mixed representation [21], of the permutation group $S_{3}$. We used, to construct a base for the channel function. the hyperspherical harmonics of the rotation group $S O(9)$, obtaining the the required mixed symmetry [21] of $S_{3}$. by means of the projection operator for this symmetry, to give for the spin $\otimes$ spatial composition a totally anti-symmetric representation. The potential curves are calculated, in this basis, for arbitrary angular momenta $\left(l_{1}, l_{2}, l_{3}\right)$ configuration, and later we add others functions in a $(0.0,0)$, or $s^{3}$, configuration, to improve the convergence for small $R$ region. In this work we treat the hyperspherical radial equation within extreme adiabatic approximation (EAA), without non-adiabatic correction, $\left(P_{\mu \nu}(R)\right.$ and $\left.Q_{\mu \nu}(R)\right)$, obtaining a first result for the ground-state energy $E_{E A A}^{(0)}$, setting a framework for future improvement of precision and calculalion of a new set of basis functions.
\end{abstract}




\section{Capítulo 1}

\section{Introdução}

A descrição quântica de sistemas moleculares e atômicos é um problema complexo. A obtenção de soluções suficientemente precisas para a equação de Schrödinger faria com que muitas das propriedades dos sistemas físicos e químicos pudessem ser reproduzidas. Porém tais sistemas não constituem uma classe de problemas solúveis, gerando um dos maiores obstáculos para o avanço da física contemporânea.

Uma inspiração para o tratamento de tais problemas foi advinda do trabalho de Born e Fock [1], 1928, introduzindo o chamado teorema adiabático, o qual foi posteriormente reanalisado por Tosio Kato [2] em 1950, demonstrando-o de forma mais geral. Em essência o teorema adiabático assegura que deve haver um invariante mecânico sob lentas variações, exercidas externamente ao sistema em questão.

Por exemplo, numa molécula diatômica a massa dos elétrons é muito menor do que a massa dos núcleos, de modo que os núcleos se movam lentamente e os elétrons rapidamente. Daí segue, em primeira hipótese, que podemos tratar os dois movimentos, dos elétrons e dos núcleos, separadamente. Para um tal sistema teremos o seguinte hamiltoniano

$$
H=H(\mathbf{R})+H_{\text {ele }}\left(r_{1}, r_{2}, \ldots, r_{N}\right)+V\left(\mathbf{R}, r_{1}, r_{2}, \ldots, r_{N}\right),
$$

onde os $r_{i} \mathrm{~s}$ são as coordenadas eletrônicas e $\mathbf{R}$ a coordenada relativa à posição entre os núcleos. No hamiltoniano acima o termo $H(\mathbf{R})$ corresponde à energia cinética dos núcleos. o termo $H_{e l e}$ corresponde à energia eletrônica do sistema e $V$ a energia de interação entre os núcleos e a energia de interação entre os elétrons e os núcieos. 
Tratamos então este problema para um valor fixo da distância $\mathbf{R}$ entre os núcleos, e determinamos o auto-estado dos elétrons $|n(\mathbf{R})\rangle, n=1,2, \ldots$, correspondente à configuração dos núcleos

$$
\left\{H_{\text {ele }}\left(r_{1}, r_{2}, \ldots, r_{N}\right)+V\left(\mathbf{R}, r_{1}, \ldots, r_{N}\right)\right\}|n(\mathbf{R})\rangle=U_{n}(\mathbf{R})|n(\mathbf{R})\rangle,
$$

onde $U_{n}(\mathbf{R})$ é a auto-energia do $n$-ésimo auto-estado. Então se considerarmos um estado $n$, com energia $U_{n}(\mathbf{R})$, quando $\mathbf{R}$ varia devido ao movimento do núcleo o sistema sempre permanece no mesmo auto-estado, formando para cada valor de $\mathbf{R}$ um sistema completo de vetores de base. Isto significa que a função de onda do sistema adapta-se instantaneamente a qualquer mudança de $\mathbf{R}$, de modo que os elétrons moveis são ditos seguir adiabáticamente o movimento do núcleo. A energia $U_{n}(\mathbf{R})$ exerce o papel do potencial de interação entre os núcleos

$$
H=H(\mathbf{R})+U_{n}(\mathbf{R})
$$

sendo $U_{n}(\mathbf{R})$ chamado de potencial adiabático. Este é um exemplo típico da aplicação do teorema adiabático, onde a variável adiabática é a distância entre os núcleos $\mathbf{R}$, variando lentamente com relação às coordenadas eletrônicas, conforme é discutido em [3].

Pode-se ainda realizar para o sistema diatômico uma aproximação conveniente. Sendo a função de onda total deste sistema dada por

$$
\Psi \equiv|\xi(\mathbf{R})\rangle|n(\mathbf{R})\rangle
$$

onde $|\xi(\mathbf{R})\rangle$ é o estado referente ao movimento do núcleos, teremos a seguinte equação de Schrödinger

$$
\left\{H(\mathbf{R})+U_{n}(\mathbf{R})\right\}|\xi(\mathbf{R})\rangle|n(\mathbf{R})\rangle=E|\xi(\mathbf{R})\rangle|n(\mathbf{R})\rangle .
$$

Desde que o operador $H(\mathbf{R})$ envolve derivadas na coordenada $\mathbf{R}$, este irá operar tanto em $|\xi\rangle$ como em $|n\rangle$. No entanto, podemos ignorar os termos da derivada em $\mathbf{R}$ nos estados eletrônicos, contanto que a amplitude de movimento dos dois núcleos seja pequena se comparada á distância de equilíbrio entre eles. Esta aproximação é conhecida como a aproximação de Born-Oppenheimer, [4].

A idéia da separação adiabática das variáveis lentas e rápidas na equação de Schrödinger para sistemas com diversos graus de liberdade tem sido aceita pela física atómica 
e molecular, gerando a base de muitos dos metodos teóricos existentes. Na formulação original de Born e Oppenheimer tal separação foi justificada pela presença de um pequeno parâmetro, a razão das massas elétron-núcleo, sendo estas idéias tomadas posteriormente de uma maneira mais geral, o teorema adiabático. Os modelos convencionais para a estrutura atômica são formulados em termos da aproximação de partículas independentes, Hartree-Fock. As deficiências deste método são usualmente corriguidas pelo cálculo de multiplas configurações, [5], para assim melhor adequar a descrição da correlação eletrônica (interações elétron-elétron).

No tratamento de sistemas multi-eletrônicos, sistemas moleculares e sólidos, está implicita a questão de como melhor se representar os efeitos da interação elétronelétron, exercendo fundamental importância para estes sistemas.

No começo dos anos 60 foi reconhecido que para estados multi-excitados, tendo alta energia de correlação, o modelo de partículas independentes se apresentou como um método deficiente. O tratamento de tais sistemas pelo uso de coordenadas individuais, como as coordenadas esféricas $r_{i}, \theta_{i}$ e $\vartheta_{i}$, é pouco eficiente quando o caráter coletivo sobrepõem-se ao aspecto individual do sistema.

Uma descrição alternativa para a estrutura atômica foi desenvolvida introduzindo num sistema de $N$ elétrons um conjunto de coordenadas que encorporam um caráter coletivo do sistema. Tais coordenadas foram apresentadas inicialmente nos trabalhos de Fock [6], em 1930, e denominadas por coordenadas hiperesféricas. Este novo conjunto de coordenadas é justamente a generalização das coordenadas esféricas para um espaço de $3 N$ dimensões. A transformação de coordenadas esféricas para hiperesféricas é feita introduzindo-se $(N-1)$ hiper-ângulos $\alpha_{i}$ e um hiper-raio $R$ nas variáveis radias esféricas, do seguinte modo

$$
\begin{aligned}
& r_{1}=R \sin \alpha_{1} \sin \alpha_{2} \cdots \sin \alpha_{N-2} \sin \alpha_{N-1} . \\
& r_{2}=R \sin \alpha_{1} \sin \alpha_{2} \cdots \sin \alpha_{N-2} \cos \alpha_{N-1} . \\
& r_{3}=R \sin \alpha_{1} \sin \alpha_{2} \cdots \cos \alpha_{N-2}, \\
& \vdots \\
& r_{N-1}=R \sin \alpha_{1} \cos \alpha_{2}, \\
& r_{N}=R \cos \alpha_{1} .
\end{aligned}
$$

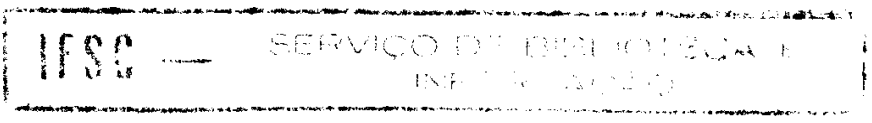


onde

$$
R=\sqrt{\sum_{j=1}^{N} r_{j}^{2}} \quad 0 \leq R \leq \infty,
$$

trocando assim as $N$ coordenadas esféricas radiais não compactas $\left(0 \leq r_{i} \leq \infty\right)$ por $N-1$ variáveis compactas $\left(0 \leq \alpha_{i} \leq \pi / 2\right)$ mais uma não compacta, $R$. O que representa uma grande simplificação do problema.

Em 1968, Macek [7] introduziu primeiramente o chamado método adiabático hiperesférico, onde o hiper-raio $R$, o qual dá a idéia de tamanho do sistema, é considerado ser adiabáticamente separavel do conjunto de coordenadas angulares. Neste metodo resolve-se a parte da equação de Schrödinger referente a energia cinética e as interações coulombianas, para valores fixos do hiper-raio, gerando assim uma dependencia da energia resultante na coordenada $R$, fazendo esta o papel de um potencial efetivo para a parte radial da equação de Schrödinger.

Nas coordenadas hiperesféricas a equação de Schrödinger para o sistema de $N$ partículas, após a exclusão convencional do centro de massa, fica

$$
\left[\frac{\partial^{2}}{\partial R^{2}}+\frac{3 N-1}{R} \frac{d}{d R}+\frac{\hat{U}(R, \Omega, N)+1 / 4}{R^{2}}+2 E\right] \Psi=0
$$

onde $\Omega$ descreve os $N-1$ hiper-ângulos e os $2 N$ ângulos esféricos. O operador $\hat{U}$ depende essêncialmente das interações coulombianas e da parte angular da energia cinética do problema.

No formalismo adiabático hiperesférico resolvemos a equação para o potencial $\hat{U}$ para um valor fixo da coordenada radial

$$
\hat{U}(R, \Omega, N) \Phi_{n}(R, \Omega)=U_{n}(R) \Phi_{n}(R, \Omega)
$$

ao admitir que a coordenada $R$ varia lentamente em comparação às coordenadas angulares. As auto-energias $U_{n}(R)$ do operador $\hat{U}$ formam, ao variarmos a coordenada $R$, as chamadas curvas de potencial, análogas ao potencial da aproximação de BornOppenheimer. As auto-funções $\Phi_{n}(R, \Omega)$ são as denominadas funções de canal, [7], formando a cada valor de $R$ um conjunto ortonormalizado de funções sob a superfície hiperesférica. 
Para a função de onda total é proposto a seguinte solução

$$
\Psi(R, \Omega)=\frac{1}{R^{(3 N-1) / 2}} \sum_{\nu} F_{\nu}(R) \Phi_{\nu}(R, \Omega)
$$

de forma a ficarmos com a seguinte equação de Schrödinger

$$
\left[\frac{\partial^{2}}{\partial R^{2}}+\frac{\hat{U}(R, \Omega, N)+1 / 4}{R^{2}}+2 E\right] \sum_{\nu} F_{\nu}(R) \Phi_{\nu}(R, \Omega)=0,
$$

onde, para o caso de física atômica o operador $\hat{U}$ é dado do seguinte modo

$$
\hat{U}(R, \Omega, N)=-\hat{\Lambda}_{3 N}^{2}(\Omega)-\frac{(3 N-1)(3 N-3)+1}{4}-2 R \hat{\mathbf{C}}(\boldsymbol{\Omega}),
$$

onde $\hat{\Lambda}_{3 N}^{2}$ é o operador de casimir para o grupo $S O(3 N)$, correspondente à parte angular da energia cinética do sistema, cuja as auto-funções são os hiperesféricos harmônicos com auto-valores

$$
\Lambda_{3 N}=\lambda_{3 N}\left(\lambda_{3 N}+3 N-2\right),
$$

e $\hat{\mathbf{C}}$ as interações coulombianas. Fórmulas gerais para sistemas nucleares de $N$ corpos são apresentadas na referência [8].

Assim sendo, pelo fato de as funções de canal dependerem de $R$, as derivadas radiais atuam nestes estados de maneira a ficarmos com a seguinte equação diferencial para as funções $F_{\mu}(R)$

$$
\left[\frac{d^{2}}{d R^{2}}+\frac{U_{\mu}(R)+1 / 4}{R^{2}}+2 E\right] F_{\mu}(R)+\sum_{\nu}\left[2 P_{\mu \nu} \frac{d}{d R}+Q_{\mu \nu}\right] F_{\nu}(R)=0
$$

onde

$$
P_{\mu \nu}(R) \equiv\left\langle\Phi_{\mu}\left|\frac{d}{d R}\right| \Phi_{\nu}\right\rangle \quad \text { e } \quad Q_{\mu \nu}(R) \equiv\left\langle\Phi_{\mu}\left|\frac{d^{2}}{d R^{2}}\right| \Phi_{\nu}\right\rangle,
$$

são os chamados acoplamentos não adiabáticos, acoplando diferentes estados eletrônicos. Podemos, a partir dos acoplamentos $P_{\mu \nu}(R)$ e $Q_{\mu \nu}(R)$, gerar algumas famílias de aproximações, introduzindo-os como correções gradativamente. A primeira delas é á aproximação adiabática extrema (Extreme Adiabatic Approximation - EAA), na qual negligenciamos todos os acoplamentos obtendo assim um caso análogo à aproximação de Born-Oppenheimer. Na aproximação adiabática desacoplada (Uncouple Adiabatic Approximation - UAA) introduzimos apenas os acoplamentos diagonais $P_{\mu \mu}$ e $Q_{\mu \mu}$. Por final temos a aproximação adiabática acoplada (Couple Adiabatic Approximation - CAA) onde consideranmos todos os acoplamentos. Para este ultimo caso, (CAA), 
estamos considerando o problema exato, sendo uma aproximação somente pelo fato de termos que truncar os cálculos do nosso problema.

Para cada uma das aproximações acima obtemos uma energia, cujo as quais se dispõem numa desigualdade denominada 'Basic Inequalities' como se segue

$$
E_{E A A} \leq E \leq E_{C A A} \leq E_{U A A}
$$

com relação à energia variacional, a qual foi demonstrada no trabalho "Proof of Basic Inequalities in the Hyperspherical Formalism for N-Body Problem", [9], nos dizendo que $E_{E A A}$ é um limite inferior e $E_{C A A}$ e $E_{U A A}$ limites superiores, qual também havia sido observada em física nuclear, mas até então sem uma demonstração rigorosa, um teorema.

O método tem a vantagem de proporcionar uma visão conceitual clara e elegante. descrevendo as propriedades desses sistemas em termos de curvas de potencial, similares as curvas de Born-Oppenheimer da física molecular. Outro aspecto interessante do método é a sua capacidade de descrever o conjunto de propriedades do sistema, sem restrições ao tipo de fenômeno a ser analisado (estados ligados, excitados ou no contínuo). As curvas de potencial e os acoplamentos não adiabáticos, contém todas as informações físicas relevantes ao problema.

A beleza do método está no fato de podermos tratar átomos e moléculas da mesma forma. através das propriedades das curvas de potencial, numa tentativa de se criar assim um método unificado.

Os trabalhos mais importantes onde este método foi estabelecido e analisado são os de U. Fano, [10], e J. Macek, [7], e posteriormente os de seus colaboradores [11] e [12]. No entanto, apesar da beleza conceitual do método, a obtenção de curvas de potencial e funções de canal precisas se revelou uma tarefa difícil. Vários outros trabalhos se dedicaram a tornar este método competitivo perante a comunidade científica. No trabalho "Two Electron Wave Function in Hyperspherical Coordinates " de J. E. Hornos , S. W. MacDowell e C. D. Caldwell, [13], procurou-se analisar a estrutura das singularidades das equações diferenciais, para assim investigar a origem das instabilidades numéricas do problema. Desta análise resultou uma metodologia capaz de produzir curvas de potencial arbitrariamente precisas e formulas que evitam derivadas 
numéricas para os coeficientes não adiabáticos, proporcionando no trabalho " Hyperspherical Adiabatic Approach for the Helium Atom " [16] a precisão almejada para sistemas de três corpos (1 ppb), relativa ao método variacional mais preciso. Outros trabalhos também analisaram o sistema de três corpos, tais como "Hyperspherical Formalism of Excitons Bound in Semiconductor Materials", [14] e "Gerenal Theoretical Approach of Coulomic Three-Body Systems by The Hyperspherical Formalism " [15], porém com resultados semi-quantitativos. A aplicação do método para diferentes sistemas de três corpos foi possível devido à visão unificada do método. Ainda outras abordagens do método para diversos sistemas de três corpos foram realizados [17].

Neste presente trabalho pretendemos dar continuidade a esta linha de pesquisa extendendo o método para aplicação em sistemas de quatro corpos, especificamente para o átomo de lítio. As curvas de potencial $U_{n}(R)$ são calculas a partir da diagonalização do operador $\hat{\mathbf{U}}$ representado no base dos hiperesféricos harmônicos com a simetria de permutação adequada para o nosso problema.

Clark e Greene [18], [19], foram os primeiros a tratar sistemas atômicos de três elétrons usando o formalismo hiperesférico. Recentemente diversos pesquisadores tem se dedicado à análise do problema de quatro corpos dentro do formalismo hiperesférico, [20]-[23], porém não há resultados para a energia não adiabática, encontrando-se os resultados ainda em um estágio inicial.

Nosso trabalho tangencia o procedimento adotado por L. Tomio [24], 1974, no que se refere à simetrização dos hiperesféricos harmônicos do $S O(9)$. O resultado obtido em [24] corresponde a um valor da energia do estado fundamental do lítio com um erro de cerca de $36 \%$, sob o valor variacional mais preciso, limitado pelo número baixo de hiperesféricos, apenas dois. O método difere do nosso a partir do tratamento das funções radiais, tratando-as variacionalmente com dois parâmetros.

Esta dissertação está organizada como se segue. No capítulo 2 estabelecemos o formalismo hiperesférico para o átomo de lítio, determinando os hiperesféricos harmônicos do $S O(9)$ advindos da parte angular da energia cinética, os quais serão usados para se determinar a base para as funções de canal. Expandimos o potencial coulombiano nas coordenadas hiperesféricas e nos esféricos harmônicos, de forma a obtermos as fórmulas necessárias para o cálculo das curvas de potencial. 
Em problemas de física atômica queremos trabalhar com funções de onda total ( Spin $\otimes$ Espacial ) anti- simétricas, para assim transcrevermos o Princípio de Exclusão de Pauli, fundamentalmente exigido para funções de onda eletrônicas. Devido o fato de os hiperesféricos harmônicos não terem simetria de permutação, isto é, não portam uma representação irredutível do grupo de permutação de três partículas, o $\mathbf{S}_{3}$, estes não represetariam uma boa escolha de base para a expanção das funções de canal, que de acordo com (1.10) devem ser anti-simétricas. Origina-se então a necessidade de investigar a simetria de permutação do nosso problema. Nos capítulos 3 e 4 desenvolvemos os aspectos conceituais necessários para o desenvolvimento e compreenção dos fundamentos do problema físico em questão, dando à dissertação um caráter autoconsistente.

A função de canal é expandida numa base composta pelo produto angular $\otimes$ spin jogando a dependência radial para os coeficientes da expansão. A simetria da parte angular é desconhecida, a princípio. Já para a parte spinorial. quando determinamos as funções de spin total, estas portam naturalmente representações irredutíveis da simetria de permutação. Logo o problema que temos em mãos é determinar qual deve ser a simetria da função angular para que possamos compor esta com a função de spin de modo a obtermos uma função anti-simétrica, já que os coeficientes da expansão são simétricos.

O átomo de lítio tem estados de spin total $1 / 2$ e 3/2. O ground state, onde será centrada a nossa atenção, e suas excitações tem spin total $1 / 2$ e, como veremos, estas portam uma representação mista irredutível do grupo de permutação $\mathbf{S}_{\mathbf{3}}$, com relação a troca de partículas. Ao compormos esta com as funções angulares iremos impor que esta última carregue a mesma representação mista irredutível do grupo $\mathbf{S}_{\mathbf{3}}$, em relação a troca das posições eletrônicas $r_{1}, r_{2}$ e $r_{3}$. Pois pode-se demonstrar (capítulo 4) que se fizermos o produto de Kronecker de duas representações irredutíveis do $\mathbf{S}_{\mathbf{3}}$. onde uma delas é uma representação mista, só obteremos uma representação anti-simétrica se a outra também carregar a mesma representação mista. E assim é mostrado no capitulo 5, onde determinamos a base angular que porta a representação mista via os operadores de projeção, previamente estabelecidos.

No capítulo 6 construimos a nossa função de onda total a partir dos coeficientes de 
Clebsh-Gordan para o produto de Kronecker das representações spinoriais e angulares.

No capítulo 7 obtemos a forma para os elementos de matriz do operador $\hat{\mathbf{U}}(R, \Omega)$ na base anti-simétrica, de forma a podemos efetuar os cálculos corretamente para as curvas de potencial. Os acoplamentos $P_{\mu \nu}$ e $Q_{\mu \nu}$ são mostrados através das expressões deduzidas em [25], encaminhando futuros cálculos para as energias não adiabáticas.

Os resultados obtidos para as bases angulares mistas do nosso problema, as curvas de potencial e os cálculos da energia adiabática extrema, $E_{E A A}^{(0)}$. são mostrados no capítulo 8 , expondo o método e deduzindo os fatores limitantes princípais.

No apêndice A fazemos a nível de ilustração um cálculo analítico para as curvas de potencial utilizando o programa algébrico Maple. 


$$
\begin{array}{ll}
\beta=\arctan \left(\frac{\sqrt{r_{1}^{2}+r_{2}^{2}}}{r_{3}}\right) & 0 \leq \beta \leq \pi / 2 \\
R=\sqrt{r_{1}^{2}+r_{2}^{2}+r_{3}^{2}} & 0 \leq R \leq \infty
\end{array}
$$

explicitando o caráter coletivo das cooerdenadas hiperesféricas. Neste novo sistema de coordenadas a coordenada $R$ dá a idéia de tamanho do sistema atômico. Assim, a equação de Schrödinger para o sistema de três elétrons pode ser expressa da seguinte forma

$$
\begin{aligned}
& \left\{\frac{\partial^{2}}{\partial R^{2}}+\frac{8}{R} \frac{\partial}{\partial R}+\frac{1}{R^{2}}\left[+\frac{1}{\sin ^{2} \beta}\left(\frac{\partial^{2}}{\partial \alpha^{2}}+(2 \cot \alpha-2 \tan \alpha) \frac{\partial}{\partial \alpha}-\frac{\mathbf{L}_{1}^{2}}{\sin ^{2} \alpha}-\right.\right.\right. \\
& \left.\left.\left.-\frac{\mathbf{L}_{2}^{2}}{\cos ^{2} \alpha}\right)+\frac{\partial^{2}}{\partial \beta^{2}}+(5 \cot \beta-2 \tan \beta) \frac{\partial}{\partial \beta}-\frac{\mathbf{L}_{3}^{2}}{\cos ^{2} \beta}-2 R \hat{\mathbf{C}}\right]+\mathbf{2 E}\right\} \mathbf{\Psi}=\mathbf{0}
\end{aligned}
$$

sendo $\Psi \equiv \Psi(R, \Omega), \Omega \equiv\left\{\alpha, \beta, \theta_{1}, \vartheta_{1}, \theta_{2}, \vartheta_{2}, \theta_{3}, \vartheta_{3}\right\}$, a função de onda total, e $\hat{\mathbf{C}}$ as interações coulombianas

$$
\begin{aligned}
\hat{\mathbf{C}} & =-\frac{Z}{\sin \alpha \sin \beta}-\frac{Z}{\cos \alpha \sin \beta}-\frac{Z}{\cos \beta}+\frac{1}{\sin \beta \sqrt{1-\sin 2 \alpha \cos \theta_{12}}} \\
& +\frac{1}{\sqrt{\sin ^{2} \beta \sin ^{2} \alpha+\cos ^{2} \beta-\sin \alpha \sin 2 \beta \cos \theta_{13}}} \\
& +\frac{1}{\sqrt{\sin ^{2} \beta \cos ^{2} \alpha+\cos ^{2} \beta-\sin 2 \beta \cos \alpha \cos \theta_{23}}},
\end{aligned}
$$

onde $\cos \theta_{i j}=\cos \theta_{i} \cos \theta_{j}+\sin \theta_{i} \sin \theta_{j} \cos \left(\vartheta_{i}-\vartheta_{j}\right)$.

Faremos uma renormalização na parte radial de nossa função de onda, de modo a eliminarmos a derivada primeira em $R$ e deixarmos a equação de Schrödinger na forma standard do formalismo hiperesférico. Fazendo-se então a transformação

$$
\Psi \longrightarrow \frac{\Psi}{R^{4}}
$$

a qual não altera as propriedades de simetria da nossa função de onda total, já que $R$ é invariante sobre rotações e permutações, teremos :

$$
\left(\frac{\partial^{2}}{\partial R^{2}}+\frac{U+1 / 4}{R^{2}}+2 E\right) \Psi=0
$$

onde para o nosso caso temos, de acordo com (1.12) :

$$
\hat{\mathbf{U}}=\hat{\mathbf{U}}(0)-2 R \hat{\mathbf{C}}
$$


sendo :

$$
\hat{\mathbf{U}}(0)=-\hat{\Lambda}_{9}^{2}-\frac{49}{4},
$$

e $\hat{\Lambda}_{\mathbf{9}}^{\mathbf{2}}$ o operador de Casimir do grupo de rotação $S O(9)$, dado por :

$$
\begin{aligned}
\hat{\mathbf{\Lambda}}_{\mathbf{9}}^{2}= & \frac{-1}{\sin ^{2} \beta}\left(\frac{\partial^{2}}{\partial \alpha^{2}}+(2 \cot \alpha-2 \tan \alpha) \frac{\partial}{\partial \alpha}-\frac{\mathbf{L}_{1}^{2}}{\sin ^{2} \alpha}-\frac{\mathbf{L}_{2}^{2}}{\cos ^{2} \alpha}\right) \\
& -\left(\frac{\partial^{2}}{\partial \beta^{2}}+(5 \cot \beta-2 \tan \beta) \frac{\partial}{\partial \beta}-\frac{\mathbf{L}_{3}^{2}}{\cos ^{2} \beta}\right) .
\end{aligned}
$$

No formalismo hiperesférico, grande parte do interesse está centrado na resolução da equação para o potencial :

$$
\hat{\mathbf{U}}(R ; \Omega) \Phi_{\nu}(R ; \Omega)=U_{\nu}(R) \Phi_{\nu}(R ; \Omega)
$$

sendo esta tratada para valores fixos da coordenada radial $R$, de modo que os autovalores e auto-funçōes do potencial venham também depender de $R$ "paramétricamente" gerando assim as "curvas de potencial", $U_{\nu}(R)$, e as "funções de canal", $\Phi_{\nu}(R ; \Omega)$, as quais a cada valor de $R$ formam um conjunto ortonormalizado de funções sob a superfície hiperesférica.

Assim sendo, a forma da função de onda total é feita levando-se em conta a semiseparabilidade entre a parte radial e a angular, expandindo esta em termos das funções de canal, da seguinte maneira

$$
\Psi(R ; \Omega)=\sum_{\nu} F_{\nu}(R) \Phi_{\nu}(R ; \Omega)
$$

Ao substituirmos esta em (2.8), obteremos a equação radial hiperesférica

$$
\left[\frac{d^{2}}{d R^{2}}+\frac{U_{\mu}(R)+1 / 4}{R^{2}}+2 E\right] F_{\mu}(R)+\sum_{\nu}\left[2 P_{\mu \nu} \frac{d}{d R}+Q_{\mu \nu}\right] F_{\nu}(R)=0
$$

onde :

$$
P_{\mu \nu}(R) \equiv\left\langle\Phi_{\mu}\left|\frac{d}{d R}\right| \Phi_{\nu}\right\rangle \quad e \quad Q_{\mu \nu}(R) \equiv\left\langle\Phi_{\mu}\left|\frac{d^{2}}{d R^{2}}\right| \Phi_{\nu}\right\rangle
$$

são os acoplamentos não adiabáticos, de acordo com o discutido na introdução.

Se olharmos agora para a forma da nossa função de onda total (2.13), veremos que pelo fato do hiper-raio ser invariante sob qualquer permutação dos elétrons, as propriedades de simetria da nossa função de onda devem estar contidas na função de canal $\Phi_{\nu}(R ; \Omega)$. Sabemos, pelo princípio de exclusão de pauli, que a nossa funçâo 
de onda deve ser anti-simétrica na troca de qualquer dois elétrons. Portanto a antisimetria assim requerida deve encontrar-se como propriedade das funções de canal. Este estudo deve ser feito sob a luz dos Grupos Finitos, mais especificamente o grupo de permutação de três partículas, $\mathbf{S}_{\mathbf{3}}$. Nasce aqui então a necessidade do aprofundamento e investigação das propriedades de simetria envolvidas na natureza do nosso problema, o que será feito nos capítulos seguintes. Prosseguiremos por enquanto apenas mencionando as propriedades da nossa função de canal, por ainda não se fazer necessário ao estabelecimento dos conceitos do formalismo hiperesférico.

Nas próximas seções discutiremos os passos em direção à determinação de uma base para as funções de canal, de modo podermos então partir para o cálculo das curvas de potencial.

\subsection{Termo Cinético. Hiperesféricos Harmônicos}

Os Hiperesféricos Harmônicos são as auto-funções do operador de casimir, os quais portam as representações irredutíveis do grupo de rotações. Para o grupo $S O(3 N)$ o operador de casimir atua nos seus respectivos hiperesféricos de modo a termos

$$
\hat{\Lambda}_{3 N}^{2} \phi^{\left[\lambda_{3 N}\right]}=\lambda_{3 N}\left(\lambda_{3 N}+3 N-2\right) \phi^{\left[\lambda_{3 N}\right]}
$$

sendo os hiperesféricos $\phi^{\left[\lambda_{3 N}\right]}$ polinômios homogêneos e harmônicos de grau $\lambda_{3 N}=$ $0,1,2,3, \ldots$

Nesta seção iremos contruir os hipersféricos harmônicos para o grupo $S O(9)$, cujo o casimir $\hat{\Lambda}_{9}^{2}$ corresponde a parte angular da energia cinético do nosso problema. Os números quânticos para o nosso problema são determinados a partir da cadeia de subgrupo do $S O(9)$, ditada pelos operadores de casimir em questão.

Para o nosso caso se escrevermos :

$$
\hat{\mathbf{\Lambda}}_{\mathbf{9}}^{2}=\frac{\hat{\mathbf{\Lambda}}_{\mathbf{6}}^{2}}{\sin ^{2} \beta}-\left(\frac{\partial^{2}}{\partial \beta^{2}}+(5 \cot \beta-2 \tan \beta) \frac{\partial}{\partial \beta}-\frac{\mathbf{L}_{3}^{2}}{\cos ^{2} \beta}\right),
$$

com :

$$
\hat{\Lambda}_{\mathbf{6}}^{2}=-\left(\frac{\partial^{2}}{\partial \alpha^{2}}+(2 \cot \alpha-2 \tan \alpha) \frac{\partial}{\partial \alpha}-\frac{\mathbf{L}_{1}^{2}}{\sin ^{2} \alpha}-\frac{\mathbf{L}_{2}^{2}}{\cos ^{2} \alpha}\right)
$$


veremos em nossa cadeia os seguintes operadores de Casimir :

$$
\begin{aligned}
& \mathbf{L}_{1}^{2} \longrightarrow S O_{1}(3) \\
& \mathbf{L}_{\mathbf{2}}^{2} \longrightarrow S O_{2}(3) \\
& \mathbf{L}_{\mathbf{3}}^{2} \longrightarrow S O_{3}(3) \\
& \mathbf{L}_{\mathbf{1 2}}^{2}=\left(\mathbf{L}_{\mathbf{1}}+\mathbf{L}_{\mathbf{2}}\right)^{2} \longrightarrow S O_{12}(3) \\
& \mathbf{L}^{2}=\left(\mathbf{L}_{\mathbf{1}}+\mathbf{L}_{\mathbf{2}}+\mathbf{L}_{\mathbf{3}}\right)^{2} \longrightarrow S O(3) \\
& \mathbf{L}_{\mathbf{z}}=\left(\mathbf{L}_{1 \mathbf{z}}+\mathbf{L}_{\mathbf{2}}+\mathbf{L}_{\mathbf{3 z}}\right) \longrightarrow U(1) \\
& \hat{\mathbf{\Lambda}}_{\mathbf{6}}^{2} \longrightarrow S O(6) \\
& \hat{\mathbf{\Lambda}}_{\mathbf{9}}^{2} \longrightarrow S O(9),
\end{aligned}
$$

de modo então a termos escolhido a seguinte cadeia e os respectivos números quânticos para seus casimires :

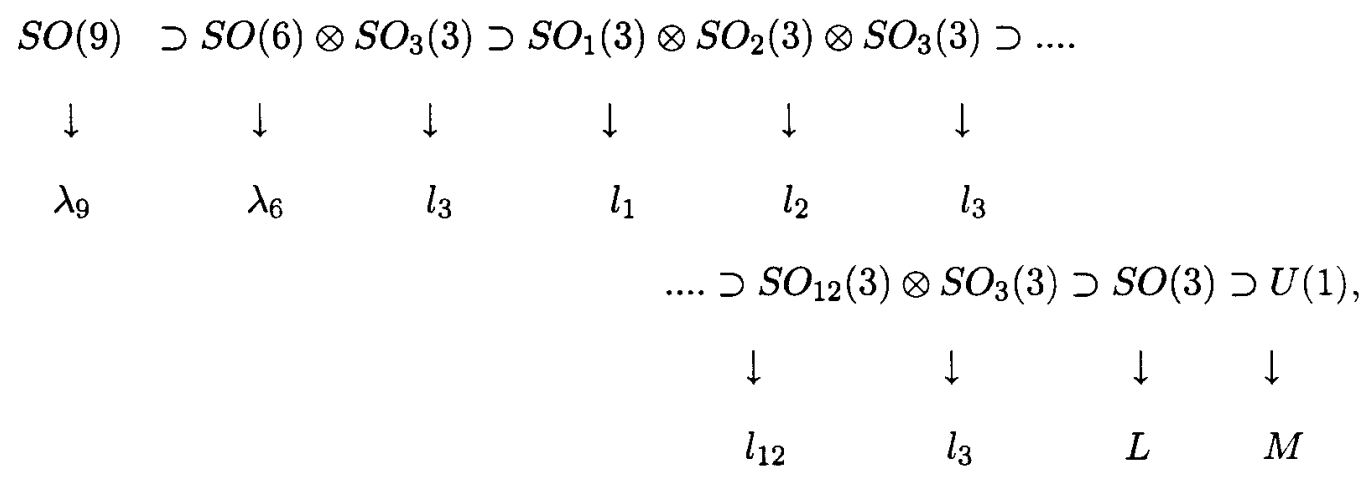

Assim sendo, denotaremos por $\phi_{\lambda_{6}\left\{l_{1} l_{2} l_{12} l_{3}\right\} L M}^{\left[\lambda_{1}\right]}$ os hiperesféricos harmônicos, os quais são polinômios homogêneos de grau $\lambda_{9} \mathrm{em} \mathbf{r}_{1}, \mathbf{r}_{2}$ e $\mathbf{r}_{3}$. O número quântico $\lambda_{9}$ está associado a um auto-valor de $\hat{\Lambda}_{\mathbf{9}}^{2}$, nos dando para cada um de seus valores uma representação irredutível para o grupo $S O(9)$, isto é, para cada auto-valor $\lambda_{9}\left(\lambda_{9}+7\right)$ teremos associado um sub-espaço formado pelos hiperesféricos com o mesmo auto-valor do casimir do $S O(9)$.

A determinação dos hiperesféricos harmônicos será feita resolvendo as equações diferenciais de auto-valor para o operador de casimir $\hat{\Lambda}_{9}^{2}$. Para o nosso problema o auto-valor que estamos interessados é o do operador $\hat{\mathbf{U}}(0)$, o qual é trivialmente relacionado com o auto-valor do operador de Casimir, de acordo com (2.10).

Assim sendo, os hiperesféricos devem ser auto funções dos operadores de Casimir da nossa 
cadeia, onde denotamos compactamente $\phi \equiv \phi_{\lambda_{6}\left\{l_{1} l_{2} l_{12} l_{3}\right\} L M}^{\left[\lambda_{9}\right]}$ :

$$
\begin{gathered}
\mathbf{L}_{i}^{2} \phi=l_{i}\left(l_{i}+1\right) \phi \quad i=1,2,3 \\
\mathbf{L}_{12}^{2} \phi=l_{12}\left(l_{12}+1\right) \phi \\
\mathbf{L}^{\mathbf{2} \phi}=L(L+1) \phi \\
\mathbf{L}_{\mathbf{z}} \phi=M \phi \\
\hat{\mathbf{\Lambda}}_{\mathbf{6}}^{\mathbf{2}} \phi=\Lambda_{6} \phi, \Lambda_{6}=\lambda_{6}\left(\lambda_{6}+4\right) \\
\hat{\mathbf{\Lambda}}_{\mathbf{9}}^{\mathbf{2}} \phi=\Lambda_{9} \phi, \Lambda_{9}=\lambda_{9}\left(\lambda_{9}+7\right)
\end{gathered}
$$

A separabilidade dos hiperesféricos é feita nos hiper-ângulos $\alpha$ e $\beta$ e os seus ângulos esféricos $\left(\Lambda_{1}, \Lambda_{2}, \Lambda_{3}\right), \Lambda_{i} \equiv\left(\theta_{i}, \vartheta_{i}\right)$, de modo a escrevermos $\phi$ da seguinte forma :

$$
\phi=g(\alpha) f(\beta) \mathcal{Y}\left(\Lambda_{1}, \Lambda_{2}, \Lambda_{3}\right)
$$

De acordo com as equações (2.19), (2.20), (2.21) e (2.22), o termo em coordenadas esféricas, $\mathcal{Y}\left(\Lambda_{1}, \Lambda_{2}, \Lambda_{3}\right)$, são obviamente os esféricos harmônicos para três partículas, $S O(3) \otimes$ $S O(3) \otimes S O(3)$, determinados partir da expansão nos Clebsch-Gordan. A parte em $\alpha$ e $\beta$ nos dá, de acordo com (2.23) e (2.24), as seguintes equações :

$$
\begin{gathered}
{\left[\frac{\partial^{2}}{\partial \alpha^{2}}+2(\cot \alpha-\tan \alpha) \frac{\partial}{\partial \alpha}-\frac{l_{1}\left(l_{1}+1\right)}{\sin ^{2} \alpha}-\frac{l_{2}\left(l_{2}+1\right)}{\cos ^{2} \alpha}\right] g(\alpha)=-\Lambda_{6} g(\alpha),} \\
{\left[\frac{\partial^{2}}{\partial \beta^{2}}+(5 \cot \beta-2 \tan \beta) \frac{\partial}{\partial \beta}-\frac{l_{3}\left(l_{3}+1\right)}{\cos ^{2} \beta}-\frac{\Lambda_{6}}{\sin ^{2} \beta}\right] f(\beta)=-\Lambda_{9} f(\beta) .}
\end{gathered}
$$

Para assim determinarmos os hiperesféricos iremos primeiramente impor uma renormalização nos hiper-ângulos, de modo que venhamos a eliminar as derivadas primeiras nas equações para $\alpha$ e $\beta$, deixando-as na forma padrão. O processo de renormalização consiste em se calcular o Jacobiano da transformação das coordenadas esféricas ( radiais )'para as coordenadas hiperesféricas, deixando assim a norma na forma canônica

$$
\int \Psi^{*} \Psi r_{1}^{2} r_{2}^{2} r_{3}^{2} d r_{1} d r_{2} d r_{3} d \Lambda_{1} d \Lambda_{2} d \Lambda_{3} \equiv \int \Psi_{R N}^{*} \Psi_{R N} d R d \alpha d \beta d \Lambda_{1} d \Lambda_{2} d \Lambda_{3}
$$

Se assim fizermos a renormalização em $\alpha$ e $\beta$ será dada por :

$$
\Psi \longrightarrow \frac{\Psi}{\sin \alpha \cos \alpha \sin ^{5 / 2} \beta \cos \beta},
$$


ou equivalentemente :

$$
f(\beta)=\frac{F(\beta)}{\sin ^{5 / 2} \beta \cos \beta},
$$

$\mathrm{e}$

$$
g(\alpha)=\frac{G(\alpha)}{\sin \alpha \cos \alpha}
$$

de modo a obtermos as novas equações :

$$
\left[\frac{\partial^{2}}{\partial \alpha^{2}}-\frac{l_{1}\left(l_{1}+1\right)}{\sin ^{2} \alpha}-\frac{l_{2}\left(l_{2}+1\right)}{\cos ^{2} \alpha}+4\right] G(\alpha)=-\Lambda_{6} G(\alpha)
$$

e

$$
\left[\frac{\partial^{2}}{\partial \beta^{2}}+\frac{\left(-\Lambda_{6}-15 / 4\right)}{\sin ^{2} \beta}-\frac{l_{3}\left(l_{3}+1\right)}{\cos ^{2} \beta}+\frac{49}{4}\right] F(\beta)=-\Lambda_{9} F(\beta),
$$

as quais são duas equações conhecidas, cuja as soluções são funções de Jacobi, dadas em [26] (pag.781, tab.22.6, item 22.6.4). De acordo com [26] temos :

$$
\left[\frac{d^{2}}{d x^{2}}+\frac{1-4 a^{2}}{16 \sin ^{2}(x / 2)}+\frac{1-4 b^{2}}{16 \cos ^{2}(x / 2)}+\left(\frac{2 n+a+b+1}{2}\right)^{2}\right] Q_{n a b}(x)=0,
$$

fazendo a seguinte transformação $x \longrightarrow 2 x$ teremos:

$$
\left[\frac{d^{2}}{d x^{2}}+\frac{1-4 a^{2}}{4 \sin ^{2} x}+\frac{1-4 b^{2}}{4 \cos ^{2} x}+(2 n+a+b+1)^{2}\right] Q_{n a b}(2 x)=0,
$$

de modo que se fizermos analogia podemos obter as soluções desejadas.

Para os auto-valores dos operadores de Casimir obtivemos :

$$
\Lambda_{6}=\left(2 \mu+l_{1}+l_{2}+2\right)^{2}-4=\lambda_{6}\left(\lambda_{6}+4\right),
$$

para o Casimir $\hat{\Lambda}_{6}^{2}$, e :

$$
\Lambda_{9}=\left(2 \nu+2 \mu+l_{1}+l_{2}+l_{3}+7 / 2\right)^{2}-\frac{49}{4}=\lambda_{9}\left(\lambda_{9}+7\right),
$$

para $\hat{\Lambda}_{\mathbf{9}}^{\mathbf{2}}$, com $\mu, \nu=0,1,2, \ldots$. Os índices $\mu$ e $\nu$ advêm das equações diferênciais, fazendo $\lambda_{6}=2 \mu+l_{1}+l_{2}$ e $\lambda_{9}=2 \nu+2 \mu+l_{1}+l_{2}+l_{3}$ deixando os auto-valores dos operadores de Casimir como de praxe na literatura dos grupos contínuos.

Podemos trocar os números quânticos $\lambda_{9}$ e $\lambda_{6}$ pelos números $\mu$ e $\nu$, de modo que ao invés de denotarmos os hiperesféricos harmônicos por $\phi \equiv \phi_{\lambda_{6}\left\{l_{1} l_{2} l_{12} l_{3}\right\} L M}^{\left[\lambda_{9}\right]}$ escreveremos $\phi_{\mu \nu l_{1} l_{2} l_{12} l_{3}}^{L M}$, de forma equivalente. Não perdemos nada ao se fazer isto, já que $\mu$ e $\nu$ são tão bons números quânticos quanto $\lambda_{9}$ e $\lambda_{6}$. Portanto, para os hiperesféricos harmônicos obtivemos :

$$
\phi_{\mu \nu l_{1} l_{2} l_{3} l_{12}}^{L M}(\alpha, \beta, \Lambda)=g_{\mu l_{1} l_{2}}(\alpha) f_{\mu \nu l_{1} l_{2} l_{3}}(\beta) \mathcal{Y}_{l_{1} l_{2} l_{3} l_{12}}^{L M}(\Lambda)
$$


com :

$$
\begin{gathered}
g_{\mu l_{1} l_{2}}(\alpha)=N_{\mu}^{l_{1} l_{2}} \sin \alpha^{l_{1}} \cos \alpha^{l_{2}} P_{\mu}^{\left(l_{1}+1 / 2, l_{2}+1 / 2\right)}(\cos 2 \alpha) \\
f_{\mu \nu l_{1} l_{2} l_{3}}(\beta)=N_{\mu \nu}^{l_{1} l_{2} l_{3}} \sin \beta^{2 \mu+l_{1}+l_{2}} \cos \beta^{l_{3}} P_{\nu}^{\left(2 \mu+l_{1}+l_{2}+2, l_{3}+1 / 2\right)}(\cos 2 \beta), \\
\mathcal{Y}_{l_{1} l_{2} l_{3} l_{12}}^{L M)=} \sum_{\substack{m_{1} m_{2} m_{3} m_{12} \\
Y_{l_{1} m_{1}}\left(\Lambda_{1}\right) Y_{l_{2} m_{2}}\left(\Lambda_{2}\right) Y_{l_{3} m_{3}}\left(\Lambda_{3}\right),}}\left\langle l_{1} m_{1} l_{2} m_{2} \mid l_{12} m_{12}\right\rangle\left\langle l_{12} m_{12} l_{3} m_{3} \mid L M\right\rangle
\end{gathered}
$$

ortonormais segundo o produto escalar :

$$
\int \phi_{\mu \nu l_{1} l_{2} l_{3} l_{12}}^{L M *} \phi_{\mu^{\prime} \nu^{\prime} l_{1}^{\prime} l_{2}^{\prime} l_{3}^{\prime} l_{12}^{\prime}}^{L M} d \Omega=\delta_{\mu \mu^{\prime}} \delta_{\nu \nu^{\prime}} \delta_{l_{1} l_{1}^{\prime}} \delta_{l_{2} l_{2}^{\prime}} \delta_{l_{3} l_{3}^{\prime}} \delta_{l_{12} l_{12}^{\prime}}
$$

sendo o elemento de volume dado por

$$
d \Omega \equiv \sin ^{2} \alpha \cos ^{2} \alpha \sin ^{5} \beta \cos ^{2} \beta d \alpha d \beta d \Lambda
$$

$\operatorname{com} d \Lambda \equiv\left\{d \Lambda_{1} d \Lambda_{2} d \Lambda_{3},\left(d \Lambda_{i}=\sin ^{2} \theta_{i} d \theta_{i} d \vartheta_{i}\right)\right\}$

Nas equações acima as grandezas $N_{\mu}^{l_{\mu} l_{2}}$ e $N_{\mu \nu}^{l_{1} l_{2} l_{3}}$ são constantes de normalização, $P_{n}^{(a, b)}$ os Polinômio de Jacobi usuais e $\mathcal{Y}_{l_{1} l_{2} l_{3} l_{12}}^{L M}(\Lambda)$ os Esféricos Harmônicos para três partículas $[S O(3) \otimes S O(3) \otimes S O(3)]$ e os coeficientes da expansão (2.40) são os coeficientes de ClebschGordan, os quais estabelecem assim as seguintes regras de seleção :

$$
\begin{aligned}
& m_{1}+m_{2}=m_{12}, \\
& m_{4}+m_{3}=M \\
& \left|l_{1}-l_{2}\right| \leq l_{12} \leq l_{1}+l_{2}, \\
& \left|l_{3}-l_{12}\right| \leq L \leq l_{3}+l_{12}
\end{aligned}
$$

As constantes de normalização $N_{\mu}^{l_{1} l_{2}}$ e $N_{\mu \nu}^{l_{1} l_{2} l_{3}}$ são determinadas a partir das propriedades dos polinômios de Jacobi :

$$
\int_{-1}^{1} \omega(x)\left[P_{n}^{(a, b)}\right]^{2} d x=\frac{2^{a+b+1}}{2 n+a+b+1} \frac{\Gamma(n+a+1) \Gamma(n+b+1)}{n ! \Gamma(n+a+b+1)},
$$

com a função peso $\omega(x)=(1-x)^{a}(1+x)^{b}$. E desta maneira obtemos :

$$
N_{\mu}^{l_{1} l_{2}}=\left[\frac{2\left(2 \mu+l_{1}+l_{2}+2\right) \mu ! \Gamma\left(\mu+l_{1}+l_{2}+2\right)}{\Gamma\left(\mu+l_{1}+3 / 2\right) \Gamma\left(\mu+l_{2}+3 / 2\right)}\right]^{1 / 2}
$$




$$
N_{\mu \nu}^{l_{1} l_{2} l_{3}}=\left[\frac{2\left(2 \nu+2 \mu+l_{1}+l_{2}+l_{3}+7 / 2\right) \nu ! \Gamma\left(2 \mu+\nu+l_{1}+l_{2}+l_{3}+7 / 2\right)}{\Gamma\left(2 \mu+\nu+l_{1}+l_{2}+3\right) \Gamma\left(\nu+l_{3}+3 / 2\right)}\right]^{1 / 2}
$$

Assim, sendo o operador $\hat{\mathrm{U}}(0)$ dado da seguinte maneira :

$$
\hat{\mathbf{U}}(0) \phi_{\mu \nu l_{1} l_{2} l_{3} l_{12}}^{L M}=\left(-\hat{\Lambda}_{\mathbf{9}}^{2}-\frac{49}{4}\right) \phi_{\mu \nu l_{1} l_{2} l_{3} l_{12}}^{L M}=U(0) \phi_{\mu \nu l_{1} l_{2} l_{3} l_{12}}^{L M}
$$

o auto-valor $U(0)$ será dado por :

$$
U(0)=-\left(2 \mu+2 \nu+l_{1}+l_{2}+l_{3}+7 / 2\right)^{2}=-\left(\lambda_{9}+7 / 2\right)^{2},
$$

de modo então a termos resolvido a parte angular da energia cinética do nosso problema. Na próxima seção iremos calcular os elementos de matriz para a interações coulombianas na base dos hiperesféricos harmônicos do $S O(9)$.

\subsection{As interações Coulombianas}

Definimos para as interações Coulombinas o operador $\hat{\mathbf{C}}$, da seguinte forma :

$$
\hat{\mathbf{C}}=\hat{\mathbf{V}}_{1}+\hat{\mathbf{V}}_{2}+\hat{\mathbf{V}}_{3}+\hat{\mathbf{V}}_{12}+\hat{\mathbf{V}}_{13}+\hat{\mathbf{V}}_{23}
$$

com :

$$
\begin{aligned}
& \hat{V}_{1}=-\frac{Z}{\sin \alpha \sin \beta}, \\
& \hat{V}_{2}=-\frac{Z}{\sin \beta \cos \alpha}, \\
& \hat{V}_{3}=-\frac{Z}{\cos \beta}, \\
& \hat{V}_{12}= \begin{cases}\sum_{l=0}^{\infty} \sum_{m=-l}^{l}\left(\frac{4 \pi}{2 l+1}\right) \frac{(\tan \alpha)^{l}}{\sin \beta \cos \alpha} Y_{l m}\left(\Lambda_{1}\right) Y_{l m}^{*}\left(\Lambda_{2}\right) & \left(r_{2}>r_{1}\right) \\
\sum_{l=0}^{\infty} \sum_{m=-l}^{l}\left(\frac{4 \pi}{2 l+1}\right) \frac{(\cot \alpha)^{l}}{\sin \beta \sin \alpha} Y_{l m}\left(\Lambda_{1}\right) Y_{l m}^{*}\left(\Lambda_{2}\right) & \left(r_{1}>r_{2}\right),\end{cases} \\
& \hat{V}_{13}= \begin{cases}\sum_{l=0}^{\infty} \sum_{m=-l}^{l}\left(\frac{4 \pi}{2 l+1}\right) \frac{(\sin \alpha \tan \beta)^{l}}{\cos \beta} Y_{l m}\left(\Lambda_{1}\right) Y_{l m}^{*}\left(\Lambda_{3}\right) & \left(r_{3}>r_{1}\right) \\
\sum_{l=0}^{\infty} \sum_{m=-l}^{l}\left(\frac{4 \pi}{2 l+1}\right) \frac{(\cot \beta)^{l}}{(\sin \alpha)^{l+1} \sin \beta} Y_{l m}\left(\Lambda_{1}\right) Y_{l m}^{*}\left(\Lambda_{3}\right) & \left(r_{1}>r_{3}\right),\end{cases}
\end{aligned}
$$




$$
\hat{V}_{23}= \begin{cases}\sum_{l=0}^{\infty} \sum_{m=-l}^{l}\left(\frac{4 \pi}{2 l+1}\right) \frac{(\cos \alpha \tan \beta)^{l}}{\cos \beta} Y_{l m}\left(\Lambda_{2}\right) Y_{l m}^{*}\left(\Lambda_{3}\right) & \left(r_{3}>r_{2}\right) \\ \sum_{l=0}^{\infty} \sum_{m=-l}^{l}\left(\frac{4 \pi}{2 l+1}\right) \frac{(\cot \beta)^{l}}{(\cos \alpha)^{l+1} \sin \beta} Y_{l m}\left(\Lambda_{2}\right) Y_{l m}^{*}\left(\Lambda_{3}\right) & \left(r_{2}>r_{3}\right),\end{cases}
$$

sendo $\hat{V}_{i}$ as interações elétron-núcleo e $\hat{V}_{i j}$ as interações elétron-elétron, deduzidas a partir da seguinte expansão :

$$
\frac{1}{\left|\mathbf{r}-\mathbf{r}^{\prime}\right|}=\sum_{l=0}^{\infty} \sum_{m=-l}^{l}\left(\frac{4 \pi}{2 l+1}\right) \frac{r_{<}^{l}}{r_{>}^{l+1}} Y_{l m}(\hat{r}) Y_{l m}^{*}\left(\hat{r}^{\prime}\right)
$$

O elemento de matriz que queremos calcular é :

$$
\begin{aligned}
& C_{\mu \nu l_{1} l_{2} l_{3} l_{12} \mu^{\prime} \nu^{\prime} l_{1}^{\prime} l_{2}^{\prime} l_{3}^{\prime} l_{12}^{\prime} l_{12}^{\prime}}^{L M}=\left\langle\phi_{\mu \nu l_{i}}^{L M}|\hat{\mathbf{C}}| \phi_{\mu^{\prime} \nu^{\prime} l_{i}^{\prime}}^{L M}\right\rangle \\
& =\int \phi_{\mu \nu l_{1} l_{2} l_{3} l_{2}}^{L M *} \hat{\mathbf{C}} \phi_{\mu^{\prime} \nu^{\prime} 1_{1}^{\prime} 1_{2}^{\prime} I_{3} 1_{12}^{\prime} 1_{12}^{\prime}}^{L} \mathrm{~d} \Omega,
\end{aligned}
$$

e devido a forma de $\hat{\mathbf{C}}$, teremos :

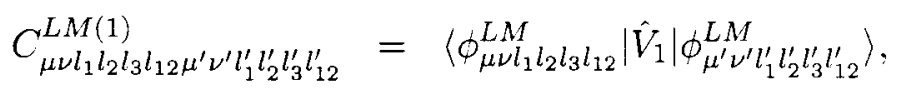

$$
\begin{aligned}
& C_{\mu \nu l_{1} l_{2} l_{3} l_{12} \mu^{\prime} \nu^{\prime} l_{1}^{\prime} l_{2}^{\prime} l_{3}^{\prime} l_{12}^{\prime}}^{L M\left(2_{12}^{\prime}\right.}=\left\langle\phi_{\mu \nu l_{1} l_{2} l_{3} l_{12}}^{L M}\left|\hat{V}_{2}\right| \phi_{\mu^{\prime} \nu^{\prime} l_{1}^{\prime} l_{2}^{\prime} l_{3}^{\prime} l_{12} l_{12}}^{L M}\right\rangle
\end{aligned}
$$

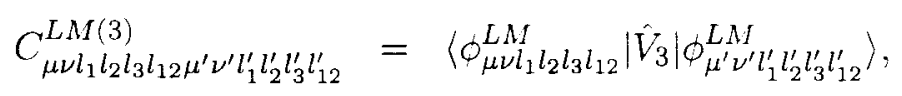

$$
\begin{aligned}
& C_{\mu \nu l_{1} l_{2} l_{3} l_{12} \mu^{\prime} \nu^{\prime} l_{1}^{\prime} l_{2}^{\prime} l_{3}^{\prime} l_{12}^{\prime}}^{L M(1)_{12}}=\left\langle\phi_{\mu \nu l_{1} l_{2} l_{3} l_{12}}^{L M}\left|\hat{V}_{12}\right| \phi_{\mu^{\prime} \nu^{\prime} l_{1}^{\prime} l_{2}^{\prime} l_{3}^{\prime} l_{12}^{\prime}}^{L M}\right\rangle, \\
& C_{\mu \nu l_{1} l_{2} l_{3} l_{2} \mu^{\prime} \nu^{\prime} l_{1}^{\prime} l_{2}^{\prime} l_{3} l_{12}^{\prime}}^{L M(13)}=\left\langle\phi_{\mu \nu l_{1} l_{2} l_{3} l_{12}}^{L M}\left|\hat{V}_{13}\right| \phi_{\left.\mu^{\prime} \nu^{\prime} l_{1}^{\prime} l_{2} l_{3} l_{32}^{\prime} l_{12}^{\prime}\right\rangle}^{L M}\right\rangle \\
& C_{\mu \nu l_{1} l_{2} l_{3} l_{12} \mu^{\prime} \nu^{\prime} l_{1}^{\prime} l_{2}^{\prime} l_{3}^{\prime} l_{12}^{\prime}}^{L M(23)}=\left\langle\phi_{\mu \nu l_{1} l_{2} l_{3} l_{12}}^{L M}\left|\hat{V}_{23}\right| \phi_{\left.\mu^{\prime} \nu^{\prime} l_{1}^{\prime} l_{2} l_{3} l_{32}^{\prime} l_{12}^{\prime}\right\rangle,}^{L M}\right\rangle
\end{aligned}
$$

implicando nas seguintes integrais :

$$
C_{\mu \nu i_{1} \mu^{\prime} \nu^{\prime} l_{2}^{\prime}}^{L M M(1)}=-Z \delta_{l_{1} l_{1}^{\prime}} \delta_{l_{2} l_{2}} \delta_{l_{3} l_{3}} \delta_{l_{12} l_{12}^{\prime}} \int_{0}^{\pi / 2}\left[\frac{g_{\mu^{\prime} l_{1} l_{2}}^{*}(\alpha) g_{\mu l_{1} l_{2}}(\alpha)}{\sin \alpha}\right] \sin ^{2} \alpha \cos ^{2} \alpha d \alpha
$$




$$
\begin{aligned}
& \int_{0}^{\pi / 2}\left[\frac{f_{\mu^{\prime} \nu^{\prime} l_{1} l_{2} l_{3}}^{*}(\beta) f_{\mu \nu l_{1} l_{2} l_{3}}(\beta)}{\sin \beta}\right] \sin ^{5} \beta \cos ^{2} \beta d \beta, \\
& C_{\mu \nu l_{i} \mu^{\prime} \nu^{\prime} l_{i}^{\prime}}^{L M(2)}=-Z \delta_{l_{1} l_{1}^{\prime}} \delta_{l_{2} l_{2}^{\prime}} \delta_{l_{3} l_{3}} \delta_{l_{12} l_{12}^{\prime}} \int_{0}^{\pi / 2}\left[\frac{g_{\mu^{\prime} l_{1} l_{2}}^{*}(\alpha) g_{\mu l_{1} l_{2}}(\alpha)}{\cos \alpha}\right] \sin ^{2} \alpha \cos ^{2} \alpha d \alpha \\
& \int_{0}^{\pi / 2}\left[\frac{f_{\mu^{\prime} \nu^{\prime} l_{1} l_{2} l_{3}}^{*}(\beta) f_{\mu \nu l_{1} l_{2} l_{3}}(\beta)}{\sin \beta}\right] \sin ^{5} \beta \cos ^{2} \beta d \beta, \\
& C_{\mu \nu l_{i} \mu^{\prime} \nu^{\prime} l_{i}^{\prime}}^{L M(3)}=-Z \delta_{l_{1} l_{1}^{\prime}} \delta_{l_{2} l_{2}} \delta_{l_{3} l_{3}^{\prime}} \delta_{l_{12} l_{12}^{\prime}} \delta_{\mu \mu^{\prime}} \int_{0}^{\pi / 2}\left[\frac{f_{\mu \nu^{\prime} l_{1} l_{2} l_{3}}^{*}(\beta) f_{\mu \nu l_{1} l_{2} l_{3}}(\beta)}{\cos \beta}\right] \\
& \sin ^{5} \beta \cos ^{2} \beta d \beta \\
& C_{\mu \nu l_{i} \mu^{\prime} \nu^{\prime} l_{i}^{\prime}}^{L M(12)}=\sum_{l}\left(\frac{4 \pi}{2 l+1}\right) \chi_{l l_{1} l_{i}^{\prime}}^{12} \int_{0}^{\pi / 2} \int_{0}^{\pi / 2} g_{\mu^{\prime} l_{1}^{\prime} l_{2}^{\prime}}^{*}(\alpha) f_{\mu^{\prime} \nu^{\prime} l_{1}^{\prime} l_{2}^{\prime} l_{3}^{\prime}}^{*}(\beta) \\
& {\left[\frac{(\sin \alpha)^{l}}{\sin \beta(\cos \alpha)^{l+1}} \Theta\left(r_{2}-r_{1}\right)+\frac{(\cos \alpha)^{l}}{\sin \beta(\sin \alpha)^{l+1}} \Theta\left(r_{1}-r_{2}\right)\right]} \\
& g_{\mu l_{1} l_{2}}(\alpha) f_{\mu \nu l_{1} l_{2} l_{3}}(\beta) \sin ^{2} \alpha \cos ^{2} \alpha \sin ^{5} \beta \cos ^{2} \beta d \alpha d \beta, \\
& \begin{aligned}
C_{\mu \nu l_{i} \mu^{\prime} \nu^{\prime} l_{i}^{\prime}}^{L M(1)}= & \sum_{l}\left(\frac{4 \pi}{2 l+1}\right) \chi_{l l_{2} l_{i}^{\prime}}^{13} \int_{0}^{\pi / 2} \int_{0}^{\pi / 2} g_{\mu^{\prime} l_{1}^{\prime} l_{2}^{\prime}}^{*}(\alpha) f_{\mu^{\prime} \nu^{\prime} l_{1}^{\prime} l_{2}^{\prime} l_{3}^{\prime}}^{*}(\beta) \\
& {\left[\frac{(\sin \alpha \sin \beta)^{l}}{(\cos \beta)^{l+1}} \Theta\left(r_{3}-r_{1}\right)+\frac{(\cos \beta)^{l}}{(\sin \alpha \sin \beta)^{l+1}} \Theta\left(r_{1}-r_{3}\right)\right] }
\end{aligned} \\
& g_{\mu l_{1} l_{2}}(\alpha) f_{\mu \nu l_{1} l_{2} l_{3}}(\beta) \sin ^{2} \alpha \cos ^{2} \alpha \sin ^{5} \beta \cos ^{2} \beta d \alpha d \beta, \\
& \begin{aligned}
C_{\mu \nu l_{i} \mu^{\prime} \nu^{\prime} l_{2}^{\prime}}^{L M(2)=} & \sum_{l}\left(\frac{4 \pi}{2 l+1}\right) \chi_{l l_{2} l_{i}^{\prime}}^{23} \int_{0}^{\pi / 2} \int_{0}^{\pi / 2} g_{\mu^{\prime} l_{1}^{\prime} l_{2}^{\prime}}^{*}(\alpha) f_{\mu^{\prime} \nu^{\prime} l_{1}^{\prime} l_{2}^{\prime} l_{3}^{l^{\prime}}}^{*}(\beta) \\
& {\left[\frac{(\cos \alpha \sin \beta)^{l}}{(\cos \beta)^{l+1}} \Theta\left(r_{3}-r_{2}\right)+\frac{(\cos \beta)^{l}}{(\cos \alpha \sin \beta)^{l+1}} \Theta\left(r_{2}-r_{3}\right)\right] }
\end{aligned} \\
& g_{\mu l_{1} l_{2}}(\alpha) f_{\mu \nu l_{1} l_{2} l_{3}}(\beta) \sin ^{2} \alpha \cos ^{2} \alpha \sin ^{5} \beta \cos ^{2} \beta d \alpha d \beta,
\end{aligned}
$$

onde fizemos :

$$
\begin{aligned}
& \chi_{l l_{2} l_{i}^{\prime}}^{12}=\int \mathcal{Y}_{l_{1}^{\prime} l_{2}^{\prime} l_{3}^{\prime} l_{12}^{\prime}}^{L^{\prime} M^{\prime}}\left(\sum_{m=-l}^{l} Y_{l m}\left(\Lambda_{1}\right) Y_{l m}^{*}\left(\Lambda_{2}\right)\right) \mathcal{Y}_{l_{1} l_{2} l_{3} l_{12}}^{L M} d \Lambda= \\
& =\delta_{L L^{\prime}} \delta_{M M^{\prime}} \delta_{l_{3} l_{3}^{\prime}} \delta_{l_{12} l_{12}^{\prime}}(-1)^{l+l_{12}}\left(\frac{2 l+1}{4 \pi}\right) \times
\end{aligned}
$$

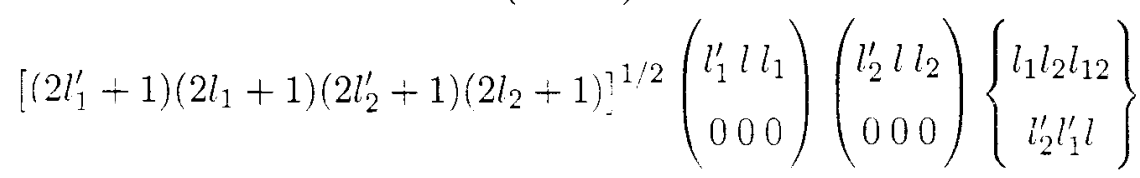




$$
\begin{aligned}
& \chi_{l l_{1} l_{i}^{\prime}}^{13}=\int \mathcal{Y}_{l_{1}^{\prime} l_{2}^{\prime} l_{3}^{\prime} l_{12}^{\prime} L_{12}^{\prime}}^{L^{\prime} x^{\prime}}\left(\sum_{m=-l}^{l} Y_{l m}\left(\Lambda_{1}\right) Y_{l m}^{*}\left(\Lambda_{3}\right)\right) \mathcal{Y}_{l_{1} l_{2} l_{3} l_{12}}^{L M} d \Lambda= \\
& =\delta_{L L^{\prime}} \delta_{M M^{\prime}} \delta_{l_{2} l_{2}}(-1)^{l_{2}+l_{12}+l_{12}^{\prime}+L}\left(\frac{2 l+1}{4 \pi}\right) \times \\
& {\left[\left(2 l_{3}^{\prime}+1\right)\left(2 l_{3}+1\right)\left(2 l_{12}^{\prime}+1\right)\left(2 l_{12}+1\right)\left(2 l_{1}^{\prime}+1\right)\left(2 l_{1}+1\right)\right]^{1 / 2} \times}
\end{aligned}
$$

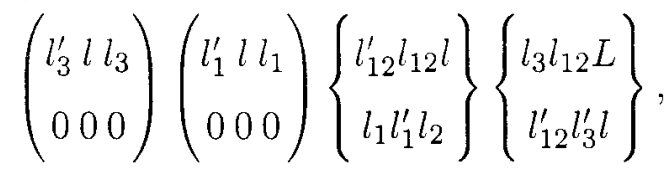

$$
\begin{aligned}
& \chi_{l l_{1} l_{i}^{\prime}}^{23}=\int \mathcal{Y}_{l_{1}^{\prime} l_{2}^{\prime} l_{3}^{\prime} l_{12}^{\prime}}^{L^{\prime} M^{\prime}}\left(\sum_{m=-l}^{l} Y_{l m}\left(\Lambda_{2}\right) Y_{l m}^{*}\left(\Lambda_{3}\right)\right) \mathcal{Y}_{l_{1} l_{2} l_{3} l_{12}}^{L M} d \Lambda= \\
& =\delta_{L L^{\prime}} \delta_{M M^{\prime}} \delta_{l_{1} l_{1}^{\prime}}(-1)^{l_{1}+l_{12}+l_{12}^{\prime}+L}\left(\frac{2 l+1}{4 \pi}\right) \times \\
& {\left[\left(2 l_{3}^{\prime}+1\right)\left(2 l_{3}+1\right)\left(2 l_{12}^{\prime}+1\right)\left(2 l_{12}+1\right)\left(2 l_{2}^{\prime}+1\right)\left(2 l_{2}+1\right)\right]^{1 / 2} \times}
\end{aligned}
$$

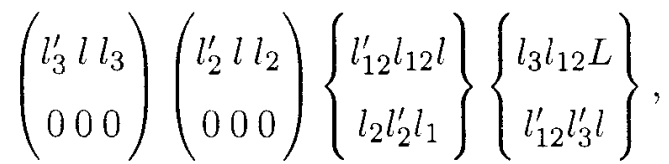

que são integrais do tipo $\int \mathcal{Y}^{*} Y Y^{*} \mathcal{Y} d \Lambda$, cujo os valores são expressos em função dos 3-j e 6-j symbols, deduzidas utilizando-se de algumas relações de [3]. Das expressões (2.53)(2.56) podemos constatar que funções com configurações de momento angular $\left(l_{1}, l_{2}, l_{3}, l_{12}\right)$ diferêntes, isto é, $l_{1} \neq l_{1}^{\prime}, l_{2} \neq l_{2}^{\prime}, \ldots$, não são acopladas pela interação coulombiana, sendo o elemento de matriz $\left\langle\phi_{\mu \nu l_{1} l_{2} l_{3} l_{12}}^{L M}|\hat{\mathbf{C}}| \phi_{\mu^{\prime} \nu^{\prime} l_{1}^{\prime} l_{2}^{\prime} l_{3} l_{12}^{\prime}}^{L M}\right\rangle$ nulo.

\subsection{Curvas de Potencial}

As curvas de potencial, $U_{n}(R)$, são os auto-valores do operador $\hat{\mathbf{U}}$, abaixo, geradas a partir da dependencia paramétrica na coordenada $R$

$$
\begin{aligned}
\hat{\mathbf{U}}(R ; \Omega) & =\frac{1}{\sin ^{2} \beta}\left(\frac{\partial^{2}}{\partial \alpha^{2}}+(2 \cot \alpha-2 \tan \alpha) \frac{\partial}{\partial \alpha}-\frac{\mathbf{L}_{1}^{2}}{\sin ^{2} \alpha}-\frac{\mathbf{L}_{2}^{2}}{\cos ^{2} \alpha}\right) \\
& +\frac{\partial^{2}}{\partial \beta^{2}}+(5 \cot \beta-2 \tan \beta) \frac{\partial}{\partial \beta}-\frac{\mathbf{L}_{3}^{2}}{\cos ^{2} \beta}+\frac{2 Z R}{\sin \alpha \sin \beta}+\frac{2 Z R}{\cos \alpha \sin \beta}-\frac{2 Z R}{\cos \beta} \\
& -\frac{2 R}{\sin \beta \sqrt{1-\sin 2 \alpha \cos \theta_{12}}}-\frac{2 R}{\sqrt{\sin ^{2} \beta \sin ^{2} \alpha+\cos ^{2} \beta-\sin \alpha \sin 2 \beta \cos \theta_{13}}} \\
& -\frac{2 R}{\sqrt{\sin ^{2} \beta \cos ^{2} \alpha+\cos ^{2} \beta-\sin 2 \beta \cos \alpha \cos \theta_{23}}}-\frac{49}{4} .
\end{aligned}
$$




\subsection{CURVAS DE POTENCIAL}

Esta é determinada resolvendo-se a equação de auto-valor para o potencial :

$$
\hat{\mathbf{U}}(R ; \Omega) \Phi_{n}(R ; \Omega)=U_{n}(R) \Phi_{n}(R ; \Omega)
$$

para um valor fixo para a coordenada radial. A função de canal será expandida numa base que deve ser auto-função de $\hat{\mathbf{U}}(0)$ onde a dependência em $R$ é jogada nos coeficientes da expansão, da seguinte forma :

$$
\Phi_{n}(R ; \Omega)=\sum_{\tau^{\prime}} \Delta_{n}^{\tau^{\prime}}(R) \varphi_{\tau^{\prime}}(\Omega)
$$

sendo que a base $\left\{\varphi_{\tau}\right\}$ deve ser obviamente anti-simétrica, equação (2.13). Aqui $\tau$ é o conjunto de números quânticos que rotula a nossa base.

Podemos a princípio resolver este problema utilizando-se como base os hiperesféricos harmônicos do grupo $S O(9)$. Porém estes não tem uma simetria de permutação definida, isto é não portam uma representação irredutivel do grupo de permutação $\mathbf{S}_{\mathbf{3}}$, não podendo nos dizer a respeito da anti-simetria com relação à troca de partículas.

No entanto, podemos construir uma base com simetria de permutação definida a partir de uma sem simetria, no caso os hiperesféricos harmônicos. E isto é feito, aqui, através do uso dos operadores de projeção, retirados da teoria dos grupos finitos. Processo este que consiste, essencialmente, em se projetar uma base sem simetria de permutação numa outra com a simetria desejada. Os operadores de projeção são uma combinação linear das permutações do grupo em questão.

As funções harmônicas com simetria de permutação podem ser expressas como combinação linear dos hiperesféricos harmônicos. Isto pode ser visto pelo fato de que a energia cinética do nosso problema é invariante sob a atuação do grupo de permutação $\mathbf{S}_{3}$ e portanto a atuação de uma permutação numa auto-função da energia cinética tem que ser também uma auto-função, com o mesmo auto-valor que a função não permutada, como pode ser visto

$$
\hat{\mathbf{U}}(0) P \phi_{\mu \nu l_{1} l_{2} l_{3} l_{12}}^{L M}=P \hat{\mathbf{U}}(0) \phi_{\mu \nu l_{1} l_{2} l_{3} l_{12}}^{L M}=-\left(\lambda_{9}+7 / 2\right)^{2} P \phi_{\mu \nu l_{1} l_{2} l_{3} l_{12}}^{L M}
$$

sendo $P$ uma permutação qualquer, de modo então que a combinação linear deve ser feita nas auto-funções com o mesmo valor de $\lambda_{9}$, isto é, nas funções do mesmo sub-espaço invariante. Assim sendo podemos utilizar os resultados para os elementos de matriz, calculados na base dos hiperesféricos, anteriormente obtidos.

As curvas de potencial apresentam um padrão de degenerescência em $R=0$, devido à forma do auto-valor $U(0)$, dado por :

$$
U(0)=-\left(2 \mu+2 \nu+l_{1}+l_{2}+l_{3}+7 / 2\right)^{2}=-\left(\lambda_{9}+7 / 2\right)^{2} .
$$


sendo $\lambda_{9}=2 \mu+2 \nu+l_{1}+l_{2}+l_{3}$. Como dissemos anteriormente a cada valor de $\lambda_{9}$, ou $U(0)$, está associada uma representação irredutível do grupo de rotação $S O(9)$. Cada representação irredutivel, forma um sub-espaço invariante com relação à atuação do operador de Casimir $\hat{\mathbf{\Lambda}}_{\mathbf{9}}^{\mathbf{2}}$, ou $\hat{\mathbf{U}}(0)$, já que estes são iguais a menos de uma constante. Este padrão de degenerescência das curvas de potencial é quebrado gradualmente para valores de $R \neq 0$, ao se incluir as interações Coulombianas, $\hat{\mathbf{U}}(R ; \Omega)=\hat{\mathbf{U}}(0)-2 R \hat{\mathbf{C}}$.

Esquematizamos abaixo um exemplo da degenerescência do operador $\hat{\mathbf{U}}$ em $R=0$ para o estado fundamental. o qual é de nosso interesse aqui

\begin{tabular}{|c|c|c|c|c|c|c|}
\hline$\lambda_{9}$ & $U(0)$ & \multicolumn{4}{|c|}{ 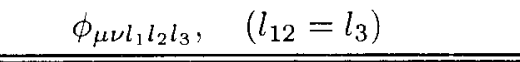 } & deg. \\
\hline 1 & -20.25 & \multicolumn{4}{|c|}{$\sim$} & 0 \\
\hline 2 & -30.25 & $\begin{array}{l}\phi_{01000} \\
\phi_{10000} \\
\phi_{00011} \\
\phi_{00101} \\
\phi_{00110} \\
\end{array}$ & & & & 5 \\
\hline 3 & -42.25 & & $\sim$ & & & 0 \\
\hline 4 & -56.25 & $\begin{array}{l}\phi_{02000} \\
\phi_{11000} \\
\phi_{20000} \\
\phi_{01011} \\
\phi_{10011} \\
\end{array}$ & $\begin{array}{l}\phi_{00022} \\
\phi_{01101} \\
\phi_{10101} \\
\phi_{01110} \\
\phi_{10110} \\
\end{array}$ & $\begin{array}{l}\phi_{00112} \\
\phi_{00121} \\
\phi_{00202} \\
\phi_{00211} \\
\phi_{00220}\end{array}$ & & 15 \\
\hline 5 & -72.25 & & $\sim$ & & & 0 \\
\hline 6 & -90.25 & $\begin{array}{c}\phi_{03000} \\
\phi_{12000} \\
\phi_{21000} \\
\phi_{30000} \\
\phi_{02011} \\
\phi_{11011} \\
\phi_{20011} \\
\phi_{01022} \\
\phi_{10022} \\
\phi_{00033}\end{array}$ & $\begin{array}{l}\phi_{02101} \\
\phi_{11101} \\
\phi_{20101} \\
\phi_{02110} \\
\phi_{11110} \\
\phi_{20110} \\
\phi_{01112} \\
\phi_{10112} \\
\phi_{01121} \\
\phi_{10121}\end{array}$ & $\begin{array}{c}\phi_{00123} \\
\phi_{00132} \\
\phi_{01202} \\
\phi_{10202} \\
\phi_{01211} \\
\phi_{10211} \\
\phi_{00213} \\
\phi_{01220} \\
\phi_{10220} \\
\phi_{00222}\end{array}$ & $\begin{array}{l}\varphi_{00231} \\
\phi_{00303} \\
\phi_{00312} \\
\varphi_{00321} \\
\varphi_{00330}\end{array}$ & 35 \\
\hline 7 & -110.25 & & $\sim$ & & & 0 \\
\hline
\end{tabular}

Tabela 2.1: Degenerescéncia das Curvas de Potencial $L=0$, em $R=0$. 
Os valores ímpares de $\lambda_{9}$ não dão funções devido à escolha de paridade $(-1)^{l_{1}+l_{2}+l_{3}}=1$, para os esféricos harmônicos $\mathcal{Y}_{l_{1} l_{2} l_{3} l_{12}}^{L M}$. O resultado acima nos diz então que para $\lambda_{9}=2$ teremos cinco curvas de potencial saindo do mesmo ponto em $R=0$, para $\lambda_{9}=4$ teremos quinze, etc.

Um labeling adequado para as curvas de potencial seria os números quânticos do operador na origem,

$$
U_{n}(R) \equiv U_{\mu \nu l_{1} l_{2} l_{3} l_{12}}^{L M}(R)
$$

mas no entanto ao projetarmos os hiperesféricos harmônicos este labeling deverá ser adaptado para o novo conjunto de funções de base na origem e que são portadores da simetria de permutação requerida ao problema. Este problema se origina essencialmente pelo fato dos operadores de projeção "misturarem" funções com diferentes números quânticos, apesar de todas terem o mesmo valor de $\lambda_{9}$. 


\section{Capítulo 3}

\section{Grupos Finitos}

Neste capítulo iremos apenas esboçar os conceitos necessários à compreenção da estrutura dos grupos finitos. Os resultados apresentados posteriormente a este capítulo estão todos ligados a estes conceitos de forma crucial e que serão utilizados para a compreenção da natureza do nosso problema. Desta maneira queremos dar um aspecto auto consistente para o nosso trabalho

\subsection{Definições. Exemplos}

Definimos por um grupo abstrato $G$ o conjunto de elementos $a, b$, $c$, etc, dotados de um operação de "multiplicação" (abstrata), de modo que o "produto" de seus elementos seja bem definido, satisfazendo as seguintes propriedades :

- Fechamento. Sendo $a$ e $b \in G$, então $a \cdot b \in G$.

- Associativa. Isto é $a \cdot(b \cdot c)=(a \cdot b) \cdot c$.

- Identidade. Existe $e \in G$ tal que, $e \cdot a=a \cdot e=a$, denominado de elemento identidade.

- Inverso. Se $a \in G$, então existe $a^{-1} \in G$ tal que, $a^{-1} \cdot a=a \cdot a^{-1}=e, a^{-1}$ é dito ser o inverso de $a$.

Do ponto de vista matemático, a multiplicação de dois elementos do grupo, $a \cdot b$, pode ser definida arbitráriamente. Porém em física estamos interessados normalmente em grupos 
onde os elementos são transformações ou operadores, de modo que o produto $R_{a} \cdot R_{b}=R_{c}$ é definido como a operação de se atuar primeiramente com $R_{b}$ e posteriormente com $R_{a}$.

Um grupo finito tem um número finito de elementos, diferentemente dos grupos contínuos , os quais seus elementos dependem de parâmetros que variam continuamente. Um grupo contínuo é um grupo infinito. A estrutura de um grupo finito é caracterizada pelo resultado da multiplicação de seus elementos, tabela do grupo, sem levar-se em conta a natureza destes.

Um grupo é dito ser Abeliano quando seus elementos comutam entre si, e portanto, $[a, b]=a \cdot b-b \cdot a=0, \forall a, b$.

Definimos como ordem do grupo $G$ o número total de elementos, denotando por $g$. Definimos como ordem do elemento $a$, o número $n$ tal que, $a^{n}=e$.

Os geradores do grupo são os elementos tais que todo elemento do grupo pode ser expresso como produto destes.

\section{EXEMPLOS :}

(1)- O conjunto de números inteiros, positivos e negativos, forma um grupo infinito, sob a operação de adição. Tendo como 0 a identidade e $-n$ o inverso de $n$.

(2)- $\mathbf{C}_{3 v}$. Formado pelas seis operações de simetria sob o triângulo :

$$
{ }_{1} \nabla_{2}^{3}
$$

sendo os elementos $\left\{e, \sigma_{1}, \sigma_{2}, \sigma_{3}, C_{3}, C_{3}^{2}\right\}$, onde os $\sigma_{i}$ são reflexões, $C_{3}$ um rotação de $120^{\circ}$ e $C_{3}^{2}$ uma rotação de $240^{\circ}$. Assim podemos contruir uma tabela de multiplicação para os elementos do grupo, a partir da lei de multiplicação para os seus elementos, dada por :

\begin{tabular}{|l||l|l|l|l|l|l|}
\hline & $\mathrm{e}$ & $\sigma_{\mathbf{3}}$ & $\sigma_{\mathbf{2}}$ & $\sigma_{\mathbf{1}}$ & $\mathbf{C}_{\mathbf{3}}$ & $\mathbf{C}_{\mathbf{3}}^{\mathbf{2}}$ \\
\hline \hline $\mathrm{e}$ & $\mathrm{e}$ & $\sigma_{3}$ & $\sigma_{2}$ & $\sigma_{1}$ & $C_{3}$ & $C_{3}^{2}$ \\
\hline$\sigma_{\mathbf{3}}$ & $\sigma_{3}$ & $\mathrm{e}$ & $C_{3}^{2}$ & $C_{3}$ & $\sigma_{1}$ & $\sigma_{2}$ \\
\hline$\sigma_{\mathbf{2}}$ & $\sigma_{2}$ & $C_{3}$ & $\mathrm{e}$ & $C_{3}^{2}$ & $\sigma 3$ & $\sigma_{1}$ \\
\hline$\sigma_{\mathbf{1}}$ & $\sigma_{1}$ & $C_{3}^{2}$ & $C_{3}$ & $\mathrm{e}$ & $\sigma_{2}$ & $\sigma_{3}$ \\
\hline $\mathbf{C}_{\mathbf{3}}$ & $C_{3}$ & $\sigma_{2}$ & $\sigma_{1}$ & $\sigma_{3}$ & $C_{3}^{2}$ & $\mathrm{e}$ \\
\hline $\mathbf{C}_{\mathbf{3}}^{2}$ & $C_{3}^{2}$ & $\sigma_{1}$ & $\sigma_{3}$ & $\sigma_{2}$ & $\mathrm{e}$ & $C_{3}$ \\
\hline
\end{tabular}

Tabela 3.1: Tabela de multiplicação do grupo $\mathbf{C}_{\mathbf{3 v}}$. 
onde temos:

$$
\begin{array}{rlll}
e_{1} \nabla_{2}^{3} & ={ }_{1} \nabla_{2}^{3}, & \sigma_{2}{ }_{1} \nabla_{2}^{3}={ }_{3} \nabla_{2}^{1}, & C_{3}{ }_{1} \nabla_{2}^{3}={ }_{3} \nabla_{1}^{2}, \\
\sigma_{1}{ }_{1} \nabla_{2}^{3}={ }_{1} \nabla_{3}^{2}, & \sigma_{3}{ }_{1} \nabla_{2}^{3}={ }_{2} \nabla_{1}^{3}, & C_{3}^{2}{ }_{1} \nabla_{2}^{3}={ }_{2} \nabla_{3}^{1},
\end{array}
$$

(3) - $\mathbf{C}_{4 v}$. Formado pelas oito operações de simetria no quadrado :

$$
{ }_{1}^{4} \square_{2}^{3}
$$

sendo os elementos do grupo $\left\{e, \sigma_{1}, \sigma_{2}, \sigma_{3}, \sigma_{4}, C_{4}\left(90^{\circ}\right), C_{4}^{2}\left(180^{\circ}\right), C_{4}^{3}\left(270^{\circ}\right)\right\}$.

(4)- Grupo cíclico, $\mathcal{C}_{n}$. Formado pelas potências de $a,\left\{e, a, a^{2}, a^{3}, \ldots, a^{n-1}\left(a^{n_{h}}=e\right)\right\}$. Por exemplo, para $n=4$, grupo cíclico de ordem quatro, temos a seguinte tabela :

\begin{tabular}{l|lll}
$\mathrm{e}$ & $\mathrm{a}$ & $\mathrm{a}^{2}$ & $\mathrm{a}^{3}$ \\
\hline $\mathrm{a}$ & $\mathrm{a}^{2}$ & $\mathrm{a}^{3}$ & $\mathrm{e}$ \\
$\mathrm{a}^{2}$ & $\mathrm{a}^{3}$ & $\mathrm{e}$ & $\mathrm{a}$ \\
$\mathrm{a}^{3}$ & $\mathrm{e}$ & $\mathrm{a}$ & $\mathrm{a}^{2}$
\end{tabular}

Tabela 3.2: Tabela de multiplicação do grupo cíclico de ordem 4.

onde $a$ é o gerador do grupo e o inverso de $a^{s}$ é $a^{r}$ tal que $r+s=n$, pois $a^{n}=e$.

(5)- Grupo Four, $\mathcal{F}$. Grupo de quatro elementos $\{e, a, b, c\}$, tal que $a^{2}=b^{2}=c^{2}=e e$ $a b=c, a c=b, b c=a$, formando assim a seguinte tabela :

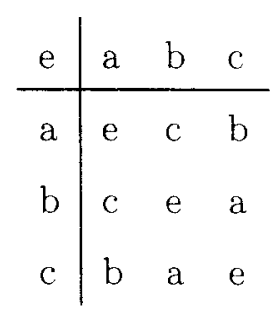

Tabela 3.3: Tabela de multiplicação do grupo four.

(6)- Grupo de Permutação, $\mathbf{S}_{n}$. Grupo formado pelas $n$ ! permutações de grau $n$ :

$$
\left(\begin{array}{cccc}
1 & 2 & \ldots & n \\
p_{1} & p_{2} & \ldots & p_{n}
\end{array}\right)
$$


O produto de duas permutações $R_{1} R_{2}$ é definido como primeiramente atuando-se com $R_{2}$ e depois com $R_{1}$. Por exemplo:

$$
R_{1}=\left(\begin{array}{ccc}
1 & 2 & 3 \\
2 & 3 & 1
\end{array}\right), R_{2}=\left(\begin{array}{ccc}
1 & 2 & 3 \\
3 & 2 & 1
\end{array}\right)
$$

de modo que :

$$
R_{1} R_{2}=\left(\begin{array}{lll}
1 & 2 & 3 \\
2 & 3 & 1
\end{array}\right)\left(\begin{array}{lll}
3 & 2 & 1 \\
1 & 2 & 3
\end{array}\right)=\left(\begin{array}{lll}
3 & 2 & 1 \\
2 & 3 & 1
\end{array}\right)=\left(\begin{array}{lll}
1 & 2 & 3 \\
1 & 3 & 2
\end{array}\right)
$$

ou genericamente, para uma permutação do $\mathbf{S}_{n}$ :

$$
R_{p}=\left(\begin{array}{cccc}
1 & 2 & \ldots & n \\
p_{1} & p_{2} & \ldots & p_{n}
\end{array}\right), R_{q}=\left(\begin{array}{cccc}
q_{1} & q_{2} & \ldots & q_{n} \\
1 & 2 & \ldots & n
\end{array}\right)
$$

de modo que :

$$
R_{p} R_{q}=\left(\begin{array}{cccc}
q_{1} & q_{2} & \ldots & q_{n} \\
p_{1} & p_{2} & \ldots & p_{n}
\end{array}\right)
$$

Podemos expressar uma permutação na forma de ciclos. Sendo uma permutação :

$$
\left(\begin{array}{llllllll}
1 & 2 & 3 & 4 & 5 & 6 & 7 & 8 \\
2 & 3 & 1 & 5 & 4 & 7 & 6 & 8
\end{array}\right)
$$

do $\mathbf{S}_{8}$, vemos que na primeira coluna troca-se 1 por 2 , na segunda 2 vai em 3 , e na terceira 3 vai em 1 fechando assim um ciclo, de modo a escrevermos (123). Se assim prosseguirmos a permutação poderá ser escrita na forma de ciclos :

$$
\left(\begin{array}{llllllll}
1 & 2 & 3 & 4 & 5 & 6 & 7 & 8 \\
2 & 3 & 1 & 5 & 4 & 7 & 6 & 8
\end{array}\right)=(123)(45)(67)
$$

onde omitimos os ciclos de apenas um símbolo, no caso (8).

Uma transposição é um ciclo de dois símbolos, $(i j)$.

Ao escrevermos uma permutação na forma de ciclos, a ordem destes é irrelevante desde que não tenham índices em comum :

$$
(123)(78)=(78)(123) \text {. }
$$


Um mesmo símbolo pode ser escrito de diversas formas :

$$
\left(a_{1} a_{2} a_{3} a_{4}\right)=\left(a_{2} a_{3} a_{4} a_{1}\right)=\left(a_{3} a_{4} a_{1} a_{2}\right)=\left(a_{4} a_{1} a_{2} a_{3}\right)
$$

A inversa de uma permutação é determinada da seguinte maneira :

$$
\left(a_{1} a_{2} a_{3} a_{4}\right)^{-1}=\left(a_{4} a_{3} a_{2} a_{1}\right)
$$

Os geradores do grupo de permutação $\mathbf{S}_{n}$ são as $n-1$ transposições adjacentes $(i, i+1)$, $i=1,2, \ldots, n-1$.

Uma permutação pode ser expressa através de transposições, por mais de uma maneira, mas o número $N$ de transposições é sempre o mesmo. Podemos então definir a paridade $\delta_{p}$ de uma permutação, por :

$$
\delta_{p}=(-1)^{N}
$$

de modo que se $\delta_{p}=1$ dizemos que a permutação é par, e se $\delta_{p}=-1$ ela é ímpar. O ciclo $\left(a_{1} a_{2} a_{3} \ldots a_{k}\right)$ tem paridade $(-1)^{k-1}$.

Podemos verificar estas propriedades tomando como exemplo o grupo $\mathbf{S}_{3}$, de ordem seis, cujo os elementos são $\{e,(12),(13),(23),(123),(132)\}$, seguindo a tabela de multiplicação do grupo :

\begin{tabular}{|c||c|c|c|c|c|c|}
\hline & e & $(\mathbf{1 2})$ & $\mathbf{( 1 3})$ & $(\mathbf{2 3})$ & $(\mathbf{1 2 3})$ & $(\mathbf{1 3 2})$ \\
\hline \hline $\mathbf{e}$ & $\mathrm{e}$ & $(\mathbf{1 2})$ & $(13)$ & $(23)$ & $(123)$ & $(132)$ \\
\hline$(\mathbf{1 2})$ & $(12)$ & $\mathrm{e}$ & $(132)$ & $(123)$ & $(23)$ & $(13)$ \\
\hline$(\mathbf{1 3})$ & $(13)$ & $(123)$ & $\mathrm{e}$ & $(132)$ & $(12)$ & $(23)$ \\
\hline$(\mathbf{2 3})$ & $(23)$ & $(132)$ & $(123)$ & $\mathrm{e}$ & $(13)$ & $(12)$ \\
\hline$(\mathbf{1 2 3})$ & $(123)$ & $(13)$ & $(23)$ & $(12)$ & $(132)$ & $\mathrm{e}$ \\
\hline$(\mathbf{1 3 2})$ & $(132)$ & $(23)$ & $(12)$ & $(13)$ & $\mathrm{e}$ & $(123)$ \\
\hline
\end{tabular}

Tabela 3.4: Tabela de multiplicação do grupo de Permutação $\mathbf{S}_{\mathbf{3}}$.

(7)- Grupo Alternado, $\mathcal{A}_{n}$. Formado pelas permutações pares de grau $n$, o qual tem $n ! / 2$ elementos. Por exemplo, grupo alternado de ordem três, formado pelas permutações pares do $\mathbf{S}_{3}, \mathcal{A}_{3} \equiv\{e,(123),(132)\}$. 


\begin{tabular}{l|ll}
$\mathrm{e}$ & $(123)$ & $(132)$ \\
\hline$(123)$ & $(132)$ & $\mathrm{e}$ \\
$(132)$ & $\mathrm{e}$ & $(123)$
\end{tabular}

Tabela 3.5: Tabela de multiplicação do grupo $\mathcal{A}_{3}$.

(8)- Grupo de ordem $8, \mathcal{H}_{8}$. Formado pelas seguintes permutações do $\mathbf{S}_{8}$ :

$$
\begin{aligned}
& h_{1}=e \quad h_{6}=(1638)(2547) \\
& h_{2}=(1234)(5678) \quad h_{7}=(1432)(5876) \\
& h_{3}=(1537)(2846) \quad h_{8}=(13)(24)(57)(68) \\
& h_{4}=(1836)(2745) \\
& h_{5}=(1735)(2648)
\end{aligned}
$$

formando a seguinte tabela :

\begin{tabular}{|l||l|l|l|l|l|l|l|l|}
\hline & $h_{1}$ & $h_{2}$ & $h_{3}$ & $h_{4}$ & $h_{5}$ & $h_{6}$ & $h_{7}$ & $h_{8}$ \\
\hline \hline$h_{1}$ & $h_{1}$ & $h_{2}$ & $h_{3}$ & $h_{4}$ & $h_{5}$ & $h_{6}$ & $h_{7}$ & $h_{8}$ \\
\hline$h_{2}$ & $h_{2}$ & $h_{8}$ & $h_{6}$ & $h_{3}$ & $h_{4}$ & $h_{5}$ & $h_{1}$ & $h_{7}$ \\
\hline$h_{3}$ & $h_{3}$ & $h_{4}$ & $h_{8}$ & $h_{7}$ & $h_{1}$ & $h_{2}$ & $h_{6}$ & $h_{5}$ \\
\hline$h_{4}$ & $h_{4}$ & $h_{5}$ & $h_{2}$ & $h_{8}$ & $h_{7}$ & $h_{1}$ & $h_{3}$ & $h_{6}$ \\
\hline$h_{5}$ & $h_{5}$ & $h_{6}$ & $h_{1}$ & $h_{2}$ & $h_{8}$ & $h_{7}$ & $h_{4}$ & $h_{3}$ \\
\hline$h_{6}$ & $h_{6}$ & $h_{3}$ & $h_{7}$ & $h_{1}$ & $h_{2}$ & $h_{8}$ & $h_{5}$ & $h_{4}$ \\
\hline$h_{7}$ & $h_{7}$ & $h_{1}$ & $h_{4}$ & $h_{5}$ & $h_{6}$ & $h_{3}$ & $h_{8}$ & $h_{2}$ \\
\hline$h_{8}$ & $h_{8}$ & $h_{7}$ & $h_{5}$ & $h_{6}$ & $h_{3}$ & $h_{4}$ & $h_{2}$ & $h_{1}$ \\
\hline
\end{tabular}

Tabela 3.6: Grupo de ordem 8, $\mathcal{H}_{8}$. 


\subsubsection{Sub-grupos. Homomorfismo e Isomorfismo}

Se existe um subconjunto $G_{s}$ de um grupo $G$ o qual também forma um grupo, sob a mesma lei de multiplicação de $G$, então $G_{s}$ é um sub-grupo de $G$, denotando-se por $G \supset G_{s}$. O próprio sub-grupo $G_{s}$ pode conter outros sub-grupos, de modo a termos :

$$
G \supset G_{s} \supset G_{s}^{\prime} \supset \ldots
$$

Exemplos : (1) Temos o que chamamos de cadeia canônica :

$$
\mathbf{S}_{n} \supset \mathbf{S}_{n-1} \supset \ldots \supset \mathbf{S}_{2}
$$

(2) O grupo de permutação $\mathbf{S}_{4}$, tem dentro de si o sub-grupo :

$$
\begin{gathered}
\mathcal{A}_{4} \equiv\{e,(123),(132), \\
(124),(142),(134),(143),(234),(243),(12)(34),(13)(24),(14)(23)\},
\end{gathered}
$$

que por sua vez contêm o grupo four, $\mathcal{F} \equiv\{e,(12)(34),(13)(24),(23)(14)\}$, de modo que

$$
\mathbf{S}_{4} \supset \mathcal{A}_{4} \supset \mathcal{F} \supset \mathbf{S}_{2}
$$

sendo o $\mathbf{S}_{2}$ formado pela identidade e uma outra permutação de dois ciclos de $\mathcal{F}$. Podiamos, dentro de $\mathcal{A}_{4}$ tomar o grupo cíclico de ordem três, $\mathcal{C}_{3} \equiv\{e,(123),(132)\}$, de modo a ficarmos com :

$$
\mathbf{S}_{4} \supset \mathcal{A}_{4} \supset \mathcal{C}_{3} \supset \mathbf{S}_{2}
$$

(3) Em $\mathrm{S}_{4}$, podemos formar um grupo cíclico, de ordem quatro, $\mathcal{C}_{4} \equiv\{e,(1234)$, (13)(24), (1432)\}, de modo a termos :

$$
\mathbf{S}_{4} \supset \mathcal{C}_{4} \supset \mathbf{S}_{2}
$$

Todo grupo tem dois sub-grupos triviais, o grupo formado somente pela identidade o próprio grupo. Estes são denominados sub-grupos impróprios. Os outros são chamados de sub-grupos próprios.

O grupo de permutação $\mathbf{S}_{n}$ é especialmente importante pois ele exausta as possíveis estruturas dos grupos finitos. Assim vemos através do teorema de Cayley :

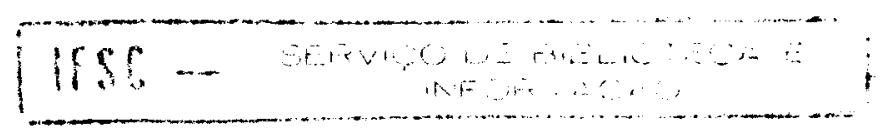


Teorema 1 Todo grupo $G$ de ordem n é isomorfo à um sub-grupo de um grupo de permutaçâo $\mathbf{S}_{n}$.

O qual é demontrado em [28]. Dizemos que dois grupos são homomorfos, $G \rightarrow G^{\prime}$, quando existe uma correspondência de cada elemento de $G$ num elemento de $G^{\prime}$, porém, tendo diversos elementos de $G$ associados o um mesmo elemento de $G^{\prime}$.

Se $G \rightarrow \mathbf{G}^{\prime}$, o conjunto de elementos de $G$ que são mapeados na identidade de $\mathbf{G}^{\prime}$ é chamado de núcleo do homomorfismo.

Exemplo: $\mathbf{C}_{3 v} \rightarrow \mathbf{S}_{2}$. De acordo com o seguinte mapeamento :

$$
\begin{gathered}
\left(e, C_{3}, C_{3}^{2}\right) \rightarrow e, \\
\left(\sigma_{1}, \sigma_{2}, \sigma_{3}\right) \rightarrow(12),
\end{gathered}
$$

sendo $\left(e, C_{3}, C_{3}^{2}\right)$ o núcleo deste homomorfismo.

Dizemos que dois grupos $G$ e $G^{\prime}$ são isomorfos, $G \cong G^{\prime}$, quando estes podem ser colocados numa correpondência $1-1$, preservando a lei de multiplicação, isto é, se em $G$ temos $a b=c$ então em $G^{\prime}$ teremos $a^{\prime} b^{\prime}=c^{\prime}$. Dois grupos isomorfos tem a mesma estrutura, porém podem ter realizações completamente diferentes.

Exemplos : (1) Observando as tabelas (3.1) e(3.4) podemos constatar o isomorfismo entre os grupos $\mathbf{C}_{3 v}$ e $\mathbf{S}_{3}$, através de correspondência

$$
\begin{aligned}
& e, C_{3} \quad, C_{3}^{2} \quad, \sigma_{1}, \sigma_{2}, \sigma_{3} \\
& e \quad,(123),(123),(23),(13),(12)
\end{aligned}
$$

e portanto $\mathbf{S}_{3} \cong \mathbf{C}_{3 v}$, tendo estes realizações diferentes.

(2) Os grupos $\mathbf{S}_{3}(124)$, formado pelos símbolos 1,2 e $4, \mathbf{S}_{3}(134)$ e $\mathbf{S}_{3}(234)$ são isomorfos ao $\mathbf{S}_{3}(123)$.

(3) O grupo $\mathcal{H}_{8}$, tabela (3.6), é conhecido como grupo dos quaternions, pelo fato de ser isomorfo á estrutura quaterniônica, a qual forma um grupo com os seguinte elementos :

$$
\begin{gathered}
1,-1, i,-i, j,-j, k,-k, \\
i^{2}=j^{2}=k^{2}=-1, i j=k, j k=i . k i=j .
\end{gathered}
$$




\subsubsection{Teorema de Lagrange}

Afim de analisar a estrutura dos grupos finitos lançamos mão de um importante teorema devido à Lagrange :

Teorema 2 A ordem de um sub-grupo de um grupo finito é um divisor da ordem do grupo.

O que seleciona apenas uma classe de sub-grupos.

Seja $G$ um grupo de ordem $g$ e $H$ um sub-grupo de ordem $h$. Se $H$ for um subgrupo impróprio então o resultado do teorema é trivial. Caso contrário podemos um elemento $a$ de $G$, que não está contido em $H$, de modo a podermos formar os produtos $e a=a, a H_{1}, a H_{2}, \ldots, a H_{h}$, com $H_{i} \in H$, denotado simbólicamente por $a H$. Os produtos $a H_{i}$ são todos diferentes, pois se $a H_{i}=a H_{j}$ teremos que $H_{i}=H_{j}$. Estes também não estão contidos em $H$, já que se $a H_{i}=H_{j}$ implica em $a=H_{j} H_{i}^{-1}$, contradizendo a nossa afirmação inicial.

Assim sendo, temos agora dois conjuntos distintos de ordem $h, H$ e $a H$, contidos em $G$. Se os elementos destes conjuntos não totalizarem o grupo $G$, podemos continuar o processo escolhendo um outro elemento $b$ de $G$, o qual não está contido em $H$ e nem em $a H$, de modo a formarmos produtos $b H_{i}$. O conjunto $b H$ nos dará $h$ novos elementos, já que $b H_{i} \notin H$, e que $b H_{i} \notin a H$, pois se $b H_{i}=a H_{j}$ então $b H_{j} H_{i}^{-1} \in a H$, contradizendo nossa afirmação a repeito de $b$. Assim fazendo até obtermos todos os elementos do grupo $G$.

Logo, podemos decompor o grupo na soma destes conjuntos :

$$
G=H+a H+b H+\ldots+k H
$$

de modo então que se $g$ é a ordem do grupo, esta é um multiplo de $h$, a ordem do sub-grupo

$$
g=m \cdot h
$$

Os conjuntos $a H$ são denominados cosets à esquerda de $H$ em $G$, os quais certamente não formam grupos. Poderiamos obter o resultado sobre a multiplicidade definindo os cosets $\dot{a}$ direita $H a$.

Como exemplo tomamos $G=\mathbf{S}_{3}$ e $H=\mathbf{S}_{2}$, de modo a termos :

$$
\mathbf{S}_{3}=\mathbf{S}_{2}+(13) \mathbf{S}_{2}=\mathbf{S}_{2}+\mathbf{S}_{2}(13)+\mathbf{S}_{2}(23)
$$

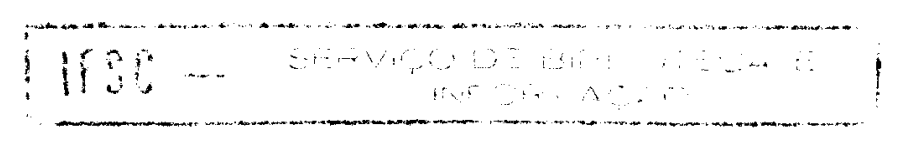


Através do resultado de Lagrange podemos concluir que se a ordem $g$ do grupo é um número primo, então o grupo não tem sub-grupos próprios.

Se escolhermos $a \in G$, podemos formar um grupo cíclico a partir das potências deste. Se $a$ é um elemento de ordem $h$, faremos $\mathcal{C}_{h}=\left\{e, a, a^{2}, \ldots, a^{h-1}\right\}$. Sendo $h$ um divisor de $g$ podemos concluir que a ordem de todos os elementos do grupo é também um divisor da ordem do grupo. E assim pelo fato de que um grupo de ordem prima não ter sub-grupos próprios, podemos concluir :

Teorema 3 Seja $G$ um grupo finito de ordem $p$, onde $p$ é um número primo, então $G$ é necessãriamente um grupo cíclico.

Resolvendo-se o problema de se determinar as possíveis estruturas dos grupos de ordem prima.

\subsubsection{Classes Conjugadas}

Dizemos que um elemento $b$ de $G$ é conjugado a um elemenro $a$ se pudemos achar um elemento $u$ de $\mathrm{G}$, tal que :

$$
b=u a u^{-1}
$$

De (3.5) podemos ver que, se $b$ é conjugado de $a, b=u a u^{-1}$, e $c$ é conjugado de $b, c=v b v^{-1}$, então $c$ é conjugado de $a$, pois :

$$
c=v b v^{-1}=v u a u^{-1} v^{-1}=(v u) a(v u)^{-1} .
$$

O conjunto de elementos conjugados entre sí formam o que denominamos classes conjugadas. Cada elemento de G está contido em uma de suas classes.

\section{Propriedades :}

(1) Em todo grupo, a identidade $e$ forma ela mesmo uma classe

(2) Num grupo Abeliano cada elemento forma uma classe :

$$
a=u a u^{-1}=u u^{-1} a=a .
$$

(3) Todos os elementos de uma mesma classe tem a mesma ordem. Se $a^{h}=e$ e $b=u a u^{-1}$ então :

$$
b^{h}=\left(u a u^{-1}\right)^{h}=u a u^{-1} u a u^{-1} u a u^{-1} \ldots=u a^{h} u^{-1}=u e u^{-1}=\epsilon .
$$


Podemos aplicar a idéia de classes para os grupos de permutação $\mathbf{S}_{n}$. É fácil ver que :

$$
b=p_{a p} p^{-1}=p(i j) p^{-1}=\left(p_{i} p_{j}\right) .
$$

Se escrevermos :

$b=\left(\begin{array}{ccccccc}1 & 2 & \ldots & i & j & \ldots & n \\ p_{1} & p_{2} & \ldots & p_{i} & p_{j} & \ldots & p_{n}\end{array}\right)\left(\begin{array}{cccccc}1 & \ldots & i & j & \ldots & n \\ 1 & \ldots & j & i & \ldots & n\end{array}\right)\left(\begin{array}{ccccccc}p_{1} & p_{2} & \ldots & p_{i} & p_{j} & \ldots & p_{n} \\ 1 & 2 & \ldots & i & j & \ldots & n\end{array}\right)$,

vemos que a permutação $\operatorname{pap}^{-1}$ representa $p_{i} \rightarrow i \rightarrow j \rightarrow p_{j}$ e $p_{j} \rightarrow j \rightarrow i \rightarrow p_{i}$ ou, equivalentemente, $p_{i} \rightarrow p_{j}$.

Para :

$$
b=p(i j k) p^{-1}=\left(p_{i} p_{j} p_{k}\right)
$$

é fácilmente estendido. Portanto :

$$
b=p(i j)(k i m \ldots) p^{-1}=p(i j) p^{-1} p(k l m \ldots) p^{-1}=\left(p_{i} p_{j}\right)(p k p l p m \ldots)
$$

de modo a concluirmos que, para um grupo de permutação, os elementos conjugados, e portanto os elementos de uma mesma classe, tem a mesma estrutura de ciclos. Assim sendo, também teremos que todos os elementos de uma mesma classe tem a mesma paridade.

Para o grupo $\mathbf{S}_{4}$ temos as seguintes classes conjugadas :

1. $e$;

2. (12), (13), (14), (23), (24), (34);

3. (12) (34), (13)(24), (14)(23);

4. (123), (132), (124), (142), (134), (143), (234), (243);

5. (1234), (1243), (1324), (1342), (1423), (1432).

As permutações do $\mathbf{S}_{n}$ operam no total de $n$ símbolos. Podemos sempre escrever as permutações na forma de ciclos independentes, por exemplo $(13)(245)(67)(8)$, de modo que $\nu_{1}$ seja o número de 1-ciclos, $\nu_{2}$ o de 2-ciclos, ..., $\nu_{n}$ o de n-ciclos. Para o exemplo anterior teremos $\nu_{1}=1, \nu_{2}=3, \nu_{3}=1$ e $\nu_{4}=\nu_{5}=\nu_{6}=\nu_{7}=\nu_{8}=0$. Desde que o número total de símbolos deve ser igual à $n$, teremos que :

$$
\nu_{1}+2 \nu_{2}+3 \nu_{3}+\ldots+n \nu_{n}=n
$$

Como sabemos, todas as permutações com mesma estrutura de ciclos forma uma classe. Entâo, para cada conjunto de soluções $\left(\nu_{1} \nu_{2} \ldots \nu_{n}\right)$ de $(3.10)$ esta representada uma classe 
portanto o número de classes é igual ao número de soluções. Se reescrevermos (3.10) da forma

$$
\begin{aligned}
\nu_{1}+\nu_{2}+\nu_{3}+\ldots+\nu_{n} & =\lambda_{1} \\
\nu_{2}+\nu_{3}+\ldots+\nu_{n} & =\lambda_{2} \\
& \vdots \\
\nu_{n} & =\lambda_{n}
\end{aligned}
$$

teremos então :

$$
\lambda_{1}+\lambda_{2}+\ldots+\lambda_{n}=n \text { e } \lambda_{1} \geq \lambda_{2} \geq \ldots \geq \lambda_{n} \geq 0
$$

a partição de $n$. Logo para cada solução de (3.10) teremos uma partição de $n,\left(\lambda_{1} \lambda_{2} \ldots \lambda_{n}\right)$. Portanto para cada partição (3.12) de $n$ teremos associada uma classe de $\mathbf{S}_{n}$, e que o número de classes é dado pelo número de partições de $n$.

Então, dada uma partição $\left(\lambda_{1} \lambda_{2} \ldots \lambda_{n}\right)$ de $n$, teremos uma classe cuja a estrutura de ciclo é determinada por :

$$
\begin{aligned}
\nu_{1} & =\lambda_{1}-\lambda_{2}, \\
\nu_{2} & =\lambda_{2}-\lambda_{3}, \\
& \vdots \\
\nu_{n} & =\lambda_{n} .
\end{aligned}
$$

Por exemplo, para a partição de $5=2+2+1+0+0=\lambda_{1}+\lambda_{2}+\ldots+\lambda_{5}$ teremos $(22100)$, ou na forma abreviada $\left(2^{2} 1\right)$, de modo que :

$$
\begin{aligned}
& \nu_{1}=0, \\
& \nu_{2}=1, \\
& \nu_{3}=1, \\
& \nu_{4}=\nu_{5}=0,
\end{aligned}
$$

portanto a estrutura e ciclos (independentes) é dada por $(i j)(k l m)$. Abaixo listamos as classes de alguns $\mathbf{S}_{n} s$ :

$$
\begin{aligned}
& \mathbf{S}_{1}:(1) ; \\
& \mathbf{S}_{2}:(2),\left(1^{2}\right) ; \\
& \mathbf{S}_{3}:(3),(21),\left(1^{3}\right) ; \\
& \mathbf{S}_{4}:(4),(31),\left(2^{2}\right),\left(21^{2}\right),\left(1^{4}\right) ; \\
& \mathbf{S}_{5}:(5),(41),(32),\left(31^{2}\right),\left(2^{2} 1\right),\left(21^{3}\right),\left(1^{5}\right)
\end{aligned}
$$


Um importante dado sob o grupo $\mathbf{S}_{n}$, no contexto de classes, é o número de classes, o qual como vimos é igual ao número de partições de $n$, e quantos elementos uma determinada classe contém. Para uma classe $\nu$ com a seguinte estrutura de ciclo $\left(\nu_{1} \nu_{2} \nu_{3} \ldots \nu_{n}\right)$, o número de elementos $g_{\nu}$ desta, é dado por :

$$
g_{\nu}=\frac{n !}{\nu_{1} ! \nu_{2} ! \nu_{3 !} ! \ldots \nu_{n} ! 1^{\nu_{1}} 2^{\nu_{2}} 3^{\nu_{3}} \ldots n^{\nu_{n}}}
$$

resultado este devido à Hamermesh, 1962, para os grupos de permutação.

\subsubsection{Sub-grupos Invariantes. Grupo Quociente}

Seja $G_{s}$ um sub-grupo de $G$, tal que os cosets à esquerda e à direita sejam iguais :

$$
a G_{s}=G_{s} a, \quad a \in G
$$

ou

$$
a G_{s} a^{-1}=G_{s}
$$

então $G_{s}$ é dito ser um sub-grupo invariante de $G$.

Através de (3.15) vemos que um sub-grupo invariante $G_{s}$ contém todos ou nenhum de seus elementos numa classe de $G$. O inverso também é verdade, se $G_{s}$ é um sub-grupo de $G$ que contém uma classe inteira de $G$, então $G_{s}$ é um sub-grupo invariante de $G$. Portanto, todo sub-grupo de um grupo Abeliano é um sub-grupo invariante.

Um grupo que não contém sub-grupos invariantes é dito ser simples. Um grupo que não contém sub-grupos invariantes e abelianos é dito ser semi-simples.

Os cosets de um sub-grupo invariante formam um grupo :

$$
\begin{aligned}
\left(a G_{s}\right)\left(b G_{s}\right) & =a\left(G_{s} b\right) G_{s}=(a b) G_{s} G_{s}=(a b) G_{s} \\
G_{s}\left(a G_{s}\right) & =\left(G_{s} a\right) G_{s}=a\left(G_{s} G_{s}\right)=a G_{s} \\
\left(a G_{s}\right)\left(a^{-1} G_{s}\right) & =G_{s}\left(a a^{-1}\right) G_{s}=G_{s},
\end{aligned}
$$

onde podemos ver que $G_{s}$ é a identidade. O grupo assim formado é denominado de grupo quociente, sendo denotado por $G / G_{s}$.

Teorema 4 Se $G$ é homomorfo à $G^{\prime}$, sendo $G_{s}$ o núcleo. então $G_{s}$ é um sub-grupo invariante e o grupo quociente $G / G_{s}$ é isomorfo à $G^{\prime}$. 
Exemplo : $\mathbf{S}_{3} / \mathcal{A}_{3}=\left\{\mathcal{A}_{3}, B\right\}$ com :

$$
\mathcal{A}_{3} \equiv\{e,(123),(132)\}, \text { e } B \equiv\{(12),(13),(23)\}
$$

tendo as relações de multiplicação :

$$
\mathcal{A}_{3} \mathcal{A}_{3}=\mathcal{A}_{3}, B B=\mathcal{A}_{3}, B \mathcal{A}_{3}=\mathcal{A}_{3} B=B
$$

e portanto $\mathbf{S}_{3} / \mathcal{A}_{3} \approx \mathbf{S}_{2} . \mathcal{A}_{3}$ é um sub-grupo invariante de $\mathbf{S}_{3}$.

\subsection{Representações}

Um representação (rep.) de um grupo $G$ é um homomorfismo de $G$ num grupo $D(G)$, cujo os elementos são operadores num espaço vetorial $L$, ou matrizes, se uma base é escolhida para $L$. A dimensão de $L$ determina a dimensão, ou grau, da representação $D(G)$.

Uma representação é definida ser fiel se o homomorfismo de $G$ em $D(G)$ se reduz à um isomorfismo.

Se $R$ e $S$ são elementos do grupo $G$, então requer-se :

$$
\begin{aligned}
D(R S) & =D(R) D(S) \\
D\left(R^{-1}\right) & =D^{-1}(R) \\
D(e) & =1 .
\end{aligned}
$$

Se $L_{n}$ é um sub-espaço invariante de $G$, então $L_{n}$ forma um espaço de representações de $G$. Alêm do mais, se uma base $\left\{\varphi_{i}\right\}$ é escolhida para $L_{n}$, as matrizes representativas de todos os elementos do grupo constituem uma representação de $G$ :

$$
R\left|\varphi_{i}\right\rangle=\sum_{j=1}^{n} D_{j i}(R)\left|\varphi_{j}\right\rangle,
$$

sendo :

$$
D_{j i}(R)=\left\langle\varphi_{j}|R| \varphi_{i}\right\rangle
$$

o elemento de matriz da representação matricial $D(R)$. 


\subsubsection{Representações Equivalentes e Caracter}

Se fizermos uma transformação de base no espaço $L_{n}$ das representações, as matrizes $D(R)$ também irão se transformar do seguinte modo :

$$
D^{\prime}(R)=C D(R) C^{-1}
$$

onde $C$ é a matriz (quadrada) que faz esta transformação. As matrizes $D^{\prime}(R)$ também provem uma representação do grupo, a qual é dita ser equivalente à representação $D(R)$.

Evidentemente, duas representações tem mesma dimensão, e duas representações com dimensões diferentes não podem ser equivalentes.

Queremos então determinar uma quantidade a qual seja intrínseca à representação, isto é, invariante sob transformações de coordenadas. No entanto, sabemos que o traço de uma matriz é invariante sob transformações de similaridade :

$$
\begin{aligned}
\operatorname{Tr}\left(D^{\prime}(R)\right) & =\sum_{i} D_{i i}^{\prime}(R)=\sum_{i k l} C_{i k} D_{k l}(R) C_{l i}^{-1}==\sum_{k l} \delta_{k l} D_{k l}(R)= \\
& =\sum_{k} D_{k k}(R)=\operatorname{Tr}(D(R)) .
\end{aligned}
$$

Em teoria de grupos, o traço de uma representação é chamado de caracter, denotando se por

$$
\chi(R)=\operatorname{Tr}(D(R))
$$

e portanto, podemos dizer que representações equivalentes tem o mesmo caracter.

Teorema 5 A igualdade dos caracters é a condição necessária e suficiente para duas representeções serem equivalentes.

Se considerarmos dois elementos conjugados, $R$ e $S$, isto é, $S=U R U^{-1}$, então $D(S)=$ $D(U) D(R) D^{-1}(U)$, de modo que teremos $\chi(S)=\chi(R)$. Portanto elementos pertencentes a uma mesma classe conjugada tem o mesmo caracter. Rotulando as classes de um grupo $G$ por $K_{1}, K_{2}, \ldots, K_{\nu}$, a representação será descrita pelo conjunto de caracters $\chi_{1}, \chi_{2}, \ldots, \chi_{\nu}$. sendo $\nu$ o número de classes de $G$. Podemos ter, no entanto, diversas representações distintas de modo a denotarmos por $\chi_{n}^{(\mu)}$ o caracter da n-ésima classe da representação $\mu$. Assim cada representação nos dá um conjunto de caracters, os quais podem ser considerados como vetores de componentes $\chi_{1}^{(\mu)}, \chi_{2}^{(\mu)}, \ldots, \chi_{\nu}^{(\mu)}$ no espaço $\nu$-dimensional. 


\subsubsection{Redutibilidade e Irredutibilidade}

Se pudemos encontrar uma transformação de base na qual todas as matrizes $D(R)$ da representação ( $n$-dimensional) fiquem da forma (equivalente) :

$$
D(R)=\left(\begin{array}{cc}
D^{(1)}(R) & 0 \\
0 & D^{(2)}(R)
\end{array}\right)
$$

onde $D^{(1)}(R)$ é uma matriz $m \times m$ e $D^{(2)}(R)$ uma matriz $(n-m) \times(n-m)$, então dizemos que representação $D(R)$ é redutível. O produto de matrizes da forma (3.23) tem sempre a mesma forma, de modo que podemos tratar $D^{(1)}(R)$ e $D^{(2)}(R)$ separadamente :

$$
\begin{aligned}
& D^{(1)}(R S)=D^{(1)}(R) D^{(1)}(S), \\
& D^{(2)}(R S)=D^{(2)}(R) D^{(2)}(S),
\end{aligned}
$$

nos dando assim duas representações, $m$-dimensional e $(n-m)$-dimensional, respectivamente. Podemos dizer então que a representação $D(R)$ foi decomposta na soma direta de $D^{(1)}(R)$ e $D^{(2)}(R)$ :

$$
D(R)=D^{(1)}(R) \oplus D^{(2)}(R)
$$

Sendo $\mathbf{x}$ um vetor no espaço $m$-dimensional da representação $D^{(1)}(R)$, a aplicação da matriz $D(R)$ neste dá :

$$
\left(\begin{array}{cc}
D^{(1)}(R) & 0 \\
0 & D^{(2)}(R)
\end{array}\right)\left(\begin{array}{l}
\mathbf{x} \\
0
\end{array}\right)=\left(\begin{array}{c}
D^{(1)}(R) \mathbf{x} \\
0
\end{array}\right),
$$

ou em outras palavras, o $m$-dimensional sub-espaço é invariante sob todas as transformações do grupo. Similarmente é o que acontece para um vetor $\mathbf{y}(n-m)$-dimensional :

$$
\left(\begin{array}{cc}
D^{(1)}(R) & 0 \\
0 & D^{(2)}(R)
\end{array}\right)\left(\begin{array}{l}
0 \\
\mathbf{y}
\end{array}\right)=\left(\begin{array}{c}
0 \\
D^{(1)}(R) \mathbf{y}
\end{array}\right),
$$

seguindo que o sub-espaço $(n-m)$-dimensional é invariante.

Assim sendo uma definição alternativa para o conceito de redutibilidade é : se no espaço das representações $L_{n}$ podemos encontrar sub-espaços invariantes sobre a ação do grupo, então $L_{n}$ é dito ser redutível, podendo ser escrito na soma direta de seus sub-espaços invariantes. Para o caso anterior, teremos portanto:

$$
L_{n}=L^{(1)} \oplus L^{(2)}
$$

Se pudermos achar outros sub-espaços invariantes dentro de $L^{(1)}\left(\mathrm{e} / \mathrm{ou} L^{(2)}\right)$, dizemos que $D^{(1)}(R)\left(\right.$ e/ou $D^{(2)}(R)$ ) ainda é redutível, se não, dizemos que $D^{(1)}(R)\left(\right.$ e/ou $D^{(2)}(R)$ ) é uma representação irredutivel (irrep), de modo que $L^{(1)}$ (e/ou $\left.L^{(2)}\right)$ é um sub-espaço irredutivel. 
Sendo então a forma geral de uma representação :

$$
D(G)=\left(\begin{array}{cccc}
D^{(1)}(R) & & & \\
& & & \\
& D^{(2)}(R) & & \\
& & \ddots & \\
& & & \\
& & D^{(k)}(G)
\end{array}\right)
$$

a qual é decomposta em suas componentes irredutíveis, de forma que :

$$
D(G)=D^{(1)}(G) \oplus D^{(2)}(G) \oplus \ldots \oplus D^{(k)}(G),
$$

e então dizemos que $D(G)$ está na forma totalmente redutível. Entre as representações irredutíveis $D^{(\nu)}(R)$ podem existir diversas representações equivalentes, de modo a escrevermos então que :

$$
D(G)=a_{1} D^{(1)}(G) \oplus a_{2} D^{(2)}(G) \oplus \ldots \oplus a_{r} D^{(r)}(G)=\sum_{\nu=1}^{r} a_{\nu} D^{(\nu)}(R),
$$

onde os $a_{\nu}$ são inteiros positivos.

Teorema 6 O número de representações irredutiveis não equivalentes de um grupo é igual ao número de classes do grupo.

O qual é demonstrado em [29]. O caracter de uma representação redutível é chamado de caracter composto $\chi(R)$, enquanto que o caracter de uma representação irredutivel é chamado de caracter simples, $\chi^{(\nu)}(R)$. De acordo com $(3.31)$, o caracter composto é expresso em termos dos caracteres simples,

$$
\chi_{i}(R)=\sum_{\nu=1}^{r} a_{\nu} \chi_{i}^{(\nu)}(R) .
$$

Segue como exemplo uma tabela genérica para os caracters simples :

\begin{tabular}{r|cccccr} 
& ${ }^{g_{1}} K_{1}$ & ${ }^{g_{2}} K_{2}$ & $\ldots$ & ${ }^{g_{i}} K_{i}$ & $\ldots$ & ${ }^{g_{k}} K_{k}$ \\
\hline$D^{(1)}$ & $\chi_{1}^{(1)}$ & $\chi_{2}^{(1)}$ & $\ldots$ & $\chi_{i}^{(1)}$ & $\ldots$ & $\chi_{k}^{(1)}$ \\
$D^{(2)}$ & $\chi_{1}^{(2)}$ & $\ldots$ & $\ldots$ & $\ldots$ & $\ldots$ & $\ldots$ \\
$\vdots$ & & & & & & $\vdots$ \\
$D^{(\mu)}$ & $\chi_{1}^{(\mu)}$ & $\ldots$ & $\ldots$ & $\chi_{i}^{(\mu)}$ & & $\chi_{k}^{(\mu)}$ \\
$\vdots$ & & & & & & $\vdots$ \\
$D^{(k)}$ & $\chi_{1}^{(k)}$ & $\chi_{2}^{(k)}$ & $\ldots$ & $\chi_{i}^{(k)}$ & $\ldots$ & $\chi_{k}^{(k)}$
\end{tabular}




\subsubsection{Representações Unitárias}

Tendo definido no espaço vetorial das representações um produto escalar, $\langle\mathbf{x} \mid \mathbf{y}\rangle$, sobre o qual os operadores de uma representação do grupo $G$ são operadores unitários :

$$
\langle U \mathbf{x} \mid U \mathbf{y}\rangle=\left\langle\mathbf{x} \mid U^{\dagger} U \mathbf{y}\right\rangle=\langle\mathbf{x} \mid \mathbf{y}\rangle
$$

isto é :

$$
U^{\dagger}=U^{-1}
$$

ou se as matrizes da representação são matrizes unitárias :

$$
D^{\dagger}(R)=D^{-1}(R)
$$

então a representação é chamada de representação unitária, a qual de acordo com (3.33) preserva a métrica do espaço.

Teorema 7 Para os grupos finitos, toda representação é equivalente a uma representaçâo unitária.

De modo então, que para grupos finitos podemos escolher nossa representação sendo unitária. 


\section{Capítulo 4}

\section{Grupos de Permutação}

Iremos neste capítulo resumir os resultados obtidos a partir da idéia de Frobenius para a determinação dos caracters dos grupos de permutação. A teoria de Frobenius para os carates exerce um papel crucial na teoria dos grupos de permutação, resolvendo completamente o problema de se encontrar as representações irredutíveis do grupo. Foge de nossa pretenção aqui deduzi-la. Um estudo profundo e detelhado é feito em [29], sendo esta a nossa fonte de referência.

\subsection{Diagramas de Young. Young Tableaux. Irreps}

Decompor um número $n$ na soma de $n$ inteiros, da forma :

$$
\begin{aligned}
n= & \lambda_{1}+\lambda_{2}+\ldots+\lambda_{n}, \\
& \lambda_{1} \geq \lambda_{2} \geq \ldots \geq \lambda_{n} \geq 0,
\end{aligned}
$$

é chamado de partição de $n$. Por exemplo, $4=4+0+0+0,4=3+1+0+0,4=2+2+0+0$, $4=2+1+1+0$ e $4=1+1+1+1$, são as partições possíveis do número 4 . Usamos o símbolo $[\lambda] \equiv\left[\lambda_{1} \lambda_{2} \ldots \lambda_{n}\right]$ para uma dada partição. De modo então que para $n=4$ teremos as seguintes partições [4000], [3100], [2200], [2110] e [1111], ou na forma abreviada [4]. [31], $\left[2^{2}\right],\left[21^{2}\right]$ e $\left[1^{4}\right]$.

$\mathrm{Na}$ teoria de grupos de permutação rotulamos as representeções irredutiveis do $\mathbf{S}_{n}$ pelas partições $[\lambda]$ de $n$. A razão pela a qual isto é feito pode ser entendida pelo fato de que uma partição $[\lambda] \equiv\left[\lambda_{1} \lambda_{2} \ldots \lambda_{n}\right]$ corresponde a uma classe $(\lambda) \equiv\left(\lambda_{1} \lambda_{2} \ldots \lambda_{n}\right)$ do $\mathbf{S}_{n}$, para o qual o número de representações irredutíveis não equivalentes é igual ao número de classes. 
Uma partição $\left[\lambda_{1} \lambda_{2} \ldots \lambda_{n}\right]$ pode ser representada graficamente por um diagrama de Young, no qual caixas são arranjadas em linhas e colunas com $\lambda_{i}$ caixas ocupando as primeiras $\lambda_{i}$ posições da $i$-ésima linha. Como por exemplo, temos para o grupo $\mathbf{S}_{4}$ as seguintes representações irredutíveis :

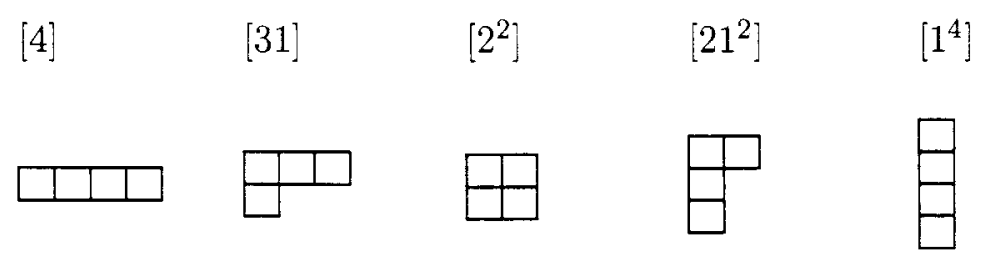

Um Young tableau é um arranjo dos números $1,2, \ldots, n$ num diagrama de Young, no qual os números aumentam quando vamos para a direita e quando vamos para baixo. Por exemplo, no diagrama :

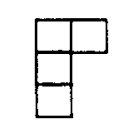

podemos colocar 1, 2, 3, 4 das seguintes maneiras :

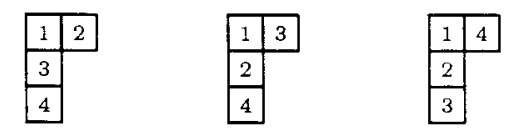

\subsection{Young Tableau. Dimensão das Irreps}

O número de possíveis Young tableaux obtidos a partir de um determinado diagrama, nos dá a dimensão da representação irredutível associada. Por exemplo, para as representações do $\mathbf{S}_{3}$ e $\mathbf{S}_{4}$ temos :

\begin{tabular}{|c|c|c|}
\hline Irrep & Young Tableaux & Dimensão \\
\hline${ }^{[3]} \square \square$ & \begin{tabular}{|l|l|l|}
1 & 2 & 3 \\
\end{tabular} & 1 \\
\hline${ }^{[21]} \square$ & \begin{tabular}{|l|l|}
1 & 2 \\
3 & \\
\end{tabular} & 2 \\
\hline [1 ${ }^{3]} \boxminus$ & \begin{tabular}{|l|}
1 \\
2 \\
3 \\
\end{tabular} & 1 \\
\hline
\end{tabular}




\begin{tabular}{|c|c|c|c|c|}
\hline Irrep & \multicolumn{3}{|c|}{ Young Tableaux } & Dimensão \\
\hline${ }^{[4]} \square \square \square$ & \begin{tabular}{|l|l|l|l|}
1 & 2 & 3 & 4 \\
\end{tabular} & & & 1 \\
\hline${ }^{[31]} \square \square$ & 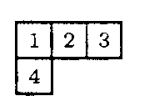 & \begin{tabular}{|l|l|}
1 & 3 \\
2 & 4 \\
\end{tabular} & \begin{tabular}{|l|l|l|}
1 & 2 & 4 \\
3 & \\
\end{tabular} & 3 \\
\hline${ }^{\left[2^{2}\right]} \square$ & \begin{tabular}{|l|l|}
1 & 2 \\
3 & 4 \\
\end{tabular} & \begin{tabular}{|l|l|}
1 & 3 \\
2 & 4 \\
\end{tabular} & & 2 \\
\hline$\left[21^{2}\right] \square$ & \begin{tabular}{|l|l|}
$\frac{1}{3}$ & 2 \\
$\frac{1}{4}$ & \\
\end{tabular} & \begin{tabular}{|l|l|}
1 & 3 \\
2 & \\
4 & \\
\end{tabular} & \begin{tabular}{|l|l|}
$\frac{4}{2}$ & 4 \\
$\frac{3}{3}$ & \\
\end{tabular} & 3 \\
\hline$\left.{ }^{[1}\right] \boxminus$ & \begin{tabular}{|l|}
$\frac{1}{2}$ \\
$\frac{3}{4}$ \\
\\
\end{tabular} & & & 1 \\
\hline
\end{tabular}

Portanto podemos associar cada Young tableau a um vetor da base da representação irredutível. Podemos determinar uma base a partir dos próprios tableaux, a qual é denominada base de Yamanouchi, mas não a trataremos aqui.

A dimensão de uma representação irredutível $[\lambda] \equiv\left[\lambda_{1} \lambda_{2} \ldots \lambda_{k}\right]$ do grupo de permutação $\mathbf{S}_{n}$ também é dada pela fórmula de Frobenius :

$$
g_{[\lambda]}=n ! \prod_{i<j \leq k}\left(\lambda_{i}-\lambda_{j}+j-i\right) / \prod_{i=1}^{k}\left(\lambda_{i}+k-i\right) !
$$

Uma fórmula simples foi dada por Robinson (1961). Na qual cada caixa de um diagrama colocamos o número de caixas à direita, da caixa em questão (incluindo a própria), mais o número de caixas abaixo, denotando este número por $l_{i}$. Assim sendo a dimensão $g_{[\lambda]}$ pode ser expressa como :

$$
g_{[\lambda]}=n ! / \prod_{i=1} l_{i}
$$

por exemplo :

$$
\begin{aligned}
& g_{[21]}=-\frac{3 !}{3 \cdot 2 \cdot 1}=2, \\
& \begin{array}{|l|l|}
\hline 3 & 1 \\
\hline 1 & \\
\hline
\end{array} \\
& g_{[31]}=\frac{4 !}{\begin{array}{|l|l|l}
4 & 2 & 1 \\
\hline 1 &
\end{array}}=\frac{4 \cdot 3 \cdot 2 \cdot 1}{4 \cdot 2 \cdot 1 \cdot 1}=3
\end{aligned}
$$




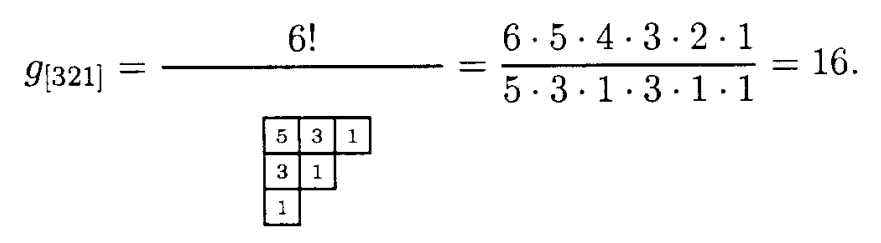

O conjunto de caracters de uma dada representação irredutível $[\lambda]$ pode ser também determinado graficamente através dos diagramas de Young. mas não exibiremos o método aqui, estando bem analisado em [29], mostraremos apenas os resultados para para os grupos $\mathbf{S}_{3}, \mathbf{S}_{4}$ e $\mathbf{S}_{5}:$

\begin{tabular}{r|rrr} 
& ${ }^{1}\left(1^{3}\right)$ & ${ }^{3}(2,1)$ & ${ }^{2}(3)$ \\
\hline$[3]$ & 1 & 1 & 1 \\
{$[2,1]$} & 2 & 0 & -1 \\
{$\left[1^{3}\right]$} & 1 & -1 & 1
\end{tabular}

\begin{tabular}{r|rrrrr} 
& ${ }^{1}\left(1^{4}\right)$ & ${ }^{6}\left(2,1^{2}\right)$ & ${ }^{3}\left(2^{2}\right)$ & ${ }^{8}(3,1)$ & ${ }^{6}(4)$ \\
\hline$[4]$ & 1 & 1 & 1 & 1 & 1 \\
{$[3,1]$} & 3 & 1 & 0 & -1 & -1 \\
{$\left[2^{2}\right]$} & 2 & 0 & -1 & 2 & 0 \\
{$\left[2,1^{2}\right]$} & 3 & -1 & 0 & -1 & 1 \\
{$\left[1^{4}\right]$} & 1 & -1 & 1 & 1 & -1
\end{tabular}

\begin{tabular}{r|rrrrrrr} 
& $1\left(1^{5}\right)$ & ${ }^{10}\left(2,1^{3}\right)$ & ${ }^{20}\left(3,1^{2}\right)$ & ${ }^{15}\left(2^{2}, 1\right)$ & ${ }^{30}(4,1)$ & ${ }^{20}(3,2)$ & ${ }^{24}(5)$ \\
\hline$[5]$ & 1 & 1 & 1 & 1 & 1 & 1 & 1 \\
{$[4,1]$} & 4 & 2 & 1 & 0 & 0 & -1 & -1 \\
{$[3,2]$} & 5 & 1 & -1 & 1 & -1 & 1 & 0 \\
{$\left[3,1^{2}\right]$} & 6 & 0 & 0 & -2 & 0 & 0 & 1 \\
{$\left[2^{2}, 1\right]$} & 5 & -1 & -1 & 1 & 1 & -1 & 0 \\
{$\left[2,1^{3}\right]$} & 4 & -2 & 1 & 0 & 0 & 1 & -1 \\
{$\left[1^{5}\right]$} & 1 & -1 & 1 & 1 & -1 & -1 & 1
\end{tabular}

Tabela 4.1: Caracters para os grupos $\mathbf{S}_{\mathbf{3}}, \mathbf{S}_{\mathbf{4}}$ e $\mathbf{S}_{\mathbf{5}}$.

\subsection{Lei de Ramificação}

Uma representação irredutível $[\lambda]$ de $\mathbf{S}_{n}$ é em geral redutível com respeito ao seu sub-grupo $\mathbf{S}_{n-1}$, do seguinte modo:

$$
D^{[\lambda]}\left(\mathbf{S}_{n}\right)=\sum_{\left[\lambda^{\prime} !\right.} D^{\left[\lambda^{\prime}\right]}\left(\mathbf{S}_{n-1}\right)
$$


onde os diagramas $\left[\lambda^{\prime}\right]$ resultam das possíveis maneiras de se remover uma caixa de $[\lambda]$.

Por exemplo a irrep [431] do $\mathbf{S}_{8}$ contém as irreps [43], [421] e $\left[3^{2} 1\right]$

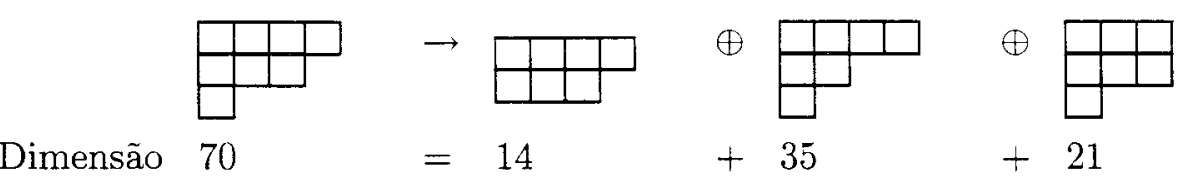

Para a forma geral temos :

$$
\left[\lambda_{1}, \lambda_{2}, \ldots, \lambda_{n}\right]=\sum_{i=n}^{1}\left[\lambda_{1}, \lambda_{2}, \ldots, \lambda_{i}-1, \ldots, \lambda_{n}\right],
$$

ou

$$
D^{\left[\lambda_{1}, \lambda_{2}, \ldots, \lambda_{n}\right]}\left(\mathbf{S}_{n}\right)=\sum_{i=n}^{1} D^{\left[\lambda_{1}, \lambda_{2}, \ldots, \lambda_{i}-1, \ldots, \lambda_{n}\right]}\left(\mathbf{S}_{n-1}\right),
$$

onde a soma é realizada para $\lambda_{i}-1 \geq 0$ e $\lambda_{i}-1>\lambda_{i+1}$. Esta é a Lei de Ramificação de uma representação irredutível do $\mathbf{S}_{n}$ em $\mathbf{S}_{n-1}$. Para os elementos de $\mathbf{S}_{n}$ que também pertencem ao $\mathbf{S}_{n-1}$ as matrizes serão bloco-diagonais. Portanto teremos para o caso anterior :

$$
D^{[431]}(R)=\left(\begin{array}{ccc}
D^{[43]}(R) & & \\
& D^{[421]}(R) & \\
& & D^{\left[3^{2} 1\right]}(R)
\end{array}\right) \quad, R \in S_{7}
$$

\subsection{Atuação do $\mathrm{S}_{n}$ em Funções de $n$ Variáveis}

Nas próximas seções iremos discutir a determinação da base que porta a representação irredutível do grupo. Para isto precisamos analisar a atuação dos operadores isomorfos aos elementos do grupo em funções arbitrárias. Denotaremos os operadores por $\mathcal{O}_{R}$. correspondendo ao elemento $R$ no grupo.

Podemos definir a ação dos elementos do grupo de duas maneiras :

$$
\begin{gathered}
\mathcal{O}_{R} \Phi(\mathbf{x})=\Phi(R \mathbf{x}) \\
\mathcal{O}_{R} \Phi(\mathbf{x})=\Phi\left(R^{-1} \mathbf{x}\right)
\end{gathered}
$$

onde $\mathbf{x}$ denota o conjunto de $n$ variáveis $\left\{x_{1}, x_{2}, \ldots, x_{n}\right\}$. Definimos também, no espaço das representações $L_{n}$, o produto escalar como sendo :

$$
\left\langle\Phi_{i} \mid \Phi_{j}\right\rangle \equiv \int \Phi_{i}^{*}(\mathbf{x}) \Phi_{j}(\mathbf{x}) d \mathbf{x}
$$


$\operatorname{com} d \mathbf{x} \equiv d^{3} x_{1} d^{3} x_{2} \ldots d^{3} x_{n}$. Assim sendo, para a atuação (4.10) teremos :

$$
\begin{aligned}
\left\langle\Phi_{i}\left|\mathcal{O}_{R}\right| \Phi_{j}\right\rangle & =\int \Phi_{i}^{*}(\mathbf{x}) \mathcal{O}_{R} \Phi_{j}(\mathbf{x}) d \mathbf{x}= \\
& =\int \Phi_{i}^{*}(\mathbf{x}) \Phi_{j}(R \mathbf{x}) d \mathbf{x}
\end{aligned}
$$

Fazendo $\mathbf{x}^{\prime}=R \mathbf{x}$ ficaremos com :

$$
\left\langle\Phi_{i}\left|\mathcal{O}_{R}\right| \Phi_{j}\right\rangle=\int \Phi_{i}^{*}\left(R^{-1} \mathbf{x}^{\prime}\right) \Phi_{j}\left(\mathbf{x}^{\prime}\right) d \mathbf{x}^{\prime}
$$

onde foi feito $R^{-1} d \mathbf{x}^{\prime}=d \mathbf{x}^{\prime}$, o que é verdade ao assumir que as variáveis tem todas o mesmo domínio, de modo que uma permutação apenas alteraria a ordem de integração. Logo :

$$
\begin{aligned}
\left\langle\Phi_{i}\left|\mathcal{O}_{R}\right| \Phi_{j}\right\rangle & =\left(\int \Phi_{i}\left(R^{-1} \mathbf{x}^{\prime}\right) \Phi_{j}^{*}\left(\mathbf{x}^{\prime}\right) d \mathbf{x}^{\prime}\right)^{*}= \\
& =\left(\int \Phi_{j}^{*}\left(\mathbf{x}^{\prime}\right) \mathcal{O}_{R^{-1}} \Phi_{i}\left(\mathbf{x}^{\prime}\right) d \mathbf{x}^{\prime}\right)^{*}= \\
& =\left\langle\Phi_{j}\left|\mathcal{O}_{R^{-1}}\right| \Phi_{i}\right\rangle^{*}
\end{aligned}
$$

ou então :

$$
\left\langle\Phi_{j}\left|\mathcal{O}_{R}\right| \Phi_{i}\right\rangle^{*}=\left\langle\Phi_{i}\left|\mathcal{O}_{R^{-1}}\right| \Phi_{j}\right\rangle
$$

o que implica em :

$$
\begin{aligned}
& D_{j i}^{*}\left(\mathcal{O}_{R}\right)=D_{i j}\left(\mathcal{O}_{R^{-1}}\right)=D_{i j}^{-1}\left(\mathcal{O}_{R}\right) \\
& D^{\dagger}\left(\mathcal{O}_{R}\right)=D^{-1}\left(\mathcal{O}_{R}\right)
\end{aligned}
$$

ou

$$
O_{R}^{\dagger}=O_{R}^{-1}
$$

Portanto, tendo definido o produto escalar (4.12) e a ação (4.10) dos elementos do grupo em $\Phi(\mathbf{x})$, a representação daí deduzida será uma representação unitária. Analogamente mostrase que a ação (4.11) também nos gera, juntamente com a definição (4.12) para o produto escalar, uma representação unitária.

A escolha da ação dos operadores do grupo está ligada à forma de multiplicação dos elementos no grupo. Podemos no grupo estabelecer dois tipos de multiplicação : esquerdadireita (E-D) e direita-esquerda (D-E). A multiplicação (E-D) implica em que no produto $R S$ atua-se primeiramente com $R$ e depois com $S$. Na multiplicação (D-E) atua-se primeiro com $S$ e depois com $R$.

A multiplicação (E-D) exige a ação $\mathcal{O}_{R} \Phi(\mathbf{x})=\Phi\left(R^{-1} \mathbf{x}\right)$ para assim estabelecer corretamente o isomorfismo entre o grupo e seus operadores. Pois para a multiplicação (E-D) teremos: 
- $\operatorname{para} \mathcal{O}_{R} \Phi(\mathbf{x})=\Phi(R \mathbf{x})$

$$
\mathcal{O}_{R} \mathcal{O}_{S} \Phi(\mathbf{x})=\mathcal{O}_{S} \Phi(R \mathbf{x})=\Phi(S(R \mathbf{x}))=\Phi(S R \mathbf{x})=\mathcal{O}_{S R} \Phi(\mathbf{x})
$$

- $\operatorname{para} \mathcal{O}_{R} \Phi(\mathbf{x})=\Phi\left(R^{-1} \mathbf{x}\right)$

$$
\mathcal{O}_{R} \mathcal{O}_{S} \Phi(\mathbf{x})=\mathcal{O}_{S} \Phi\left(R^{-1} \mathbf{x}\right)=\Phi\left(S^{-1}\left(R^{-1} \mathbf{x}\right)\right)=\Phi\left((R S)^{-1} \mathbf{x}\right)=\mathcal{O}_{R S} \Phi(\mathbf{x})
$$

onde só obtemos o isomorfismo corretamente na segunda forma.

Por outro lado, a multiplicação (D-E) exige a ação $\mathcal{O}_{R} \Phi(\mathbf{x})=\Phi(R \mathbf{x})$, para obtermos o isomorfismo. Pois para a multiplicação (D-E) teremos :

- $\operatorname{para} \mathcal{O}_{R} \Phi(\mathbf{x})=\Phi(R \mathbf{x})$

$$
\mathcal{O}_{R} \mathcal{O}_{S} \Phi(\mathbf{x})=\mathcal{O}_{R} \Phi(S \mathbf{x})=\Phi(R(S \mathbf{x}))=\Phi(R S \mathbf{x})=\mathcal{O}_{R S} \Phi(\mathbf{x})
$$

- $\operatorname{para} \mathcal{O}_{R} \Phi(\mathbf{x})=\Phi\left(R^{-1} \mathbf{x}\right)$

$$
\mathcal{O}_{R} \mathcal{O}_{S} \Phi(\mathbf{x})=\mathcal{O}_{R} \Phi\left(S^{-1} \mathbf{x}\right)=\Phi\left(R^{-1}\left(S^{-1} \mathbf{x}\right)\right)=\Phi\left((S R)^{-1} \mathbf{x}\right)=\mathcal{O}_{S R} \Phi(\mathbf{x})
$$

No nosso trabalho adotamos a multiplicação (D-E) para o grupo, o que implica na ação $\mathcal{O}_{R} \Phi(\mathbf{x})=\Phi(R \mathbf{x})$, por ser a mais usual e intuitiva. Denotaremos no texto, daqui em diante. os operadores $\mathcal{O}_{R}$ pelo símbolo do grupo $R$, simplificando assim a nossa notação :

$$
R \Phi(\mathbf{x})=\Phi(R \mathbf{x})
$$

\section{5 Álgebra do Grupo. Operadores de Young}

Este método é baseado a partir da análise de uma álgebra finita para achar as representações irredutíveis do grupo.

A partir da análise da estrutura da álgebra do grupo podemos determinar um conjunto de operadores, os quais são os geradores da álgebra. que nos provem uma base para as representações irredutíveis do grupo em questão. 


\subsubsection{Representação Regular}

Construimos a representação regular a partir de uma função $\Phi$ sem simetria nenhuma, de modo que ação dos $g$ elementos do grupo $G$, nos dá $g$ funções linearmente independentes. De modo então :

$$
\varphi_{a}=R_{a} \Phi, \quad a=1,2, \ldots g
$$

com

$$
\left\langle\varphi_{a} \mid \varphi_{b}\right\rangle=\delta_{a b}
$$

A representação que $\left\{\varphi_{R_{a}}\right\}$ porta é chamada de representação regular :

$$
R_{c} \varphi_{b}=\sum_{a=1}^{g} D_{a b}\left(R_{c}\right) \varphi_{a}
$$

e se reescrevermos esta última como:

$$
R_{c} R_{b} \Phi=\sum_{a=1}^{g} D_{a b}\left(R_{c}\right) R_{a} \Phi
$$

veremos que os elementos de matriz são dados por :

$$
D_{a b}\left(R_{c}\right)= \begin{cases}1 & , \text { se } R_{c} R_{b}=R_{a} \\ 0 & , \text { caso contrário }\end{cases}
$$

Portanto para a representação regular, vemos que em todo linha e toda coluna há somente um elemento não nulo, e também que os elementos da diagonal são todos nulos, excluindo a matriz identidade. Por exemplo, com relação ao $\mathbf{S}_{3}$, a função $|\alpha \beta \gamma\rangle$ não tem simetria nenhuma, de modo a termos a seguinte representação, regular, para o elemento (23) do grupo :

$$
D(23)=\left(\begin{array}{llllll}
0 & 0 & 0 & 1 & 0 & 0 \\
0 & 0 & 0 & 0 & 0 & 1 \\
0 & 0 & 0 & 0 & 1 & 0 \\
1 & 0 & 0 & 0 & 0 & 0 \\
0 & 1 & 0 & 0 & 0 & 0 \\
0 & 0 & 1 & 0 & 0 & 0
\end{array}\right)
$$

Teorema 8 A representação regular é totalmente redutivel e contém cada representaçấo irredutivel um número de vezes igual à dimensão da representaçâo.

Por exemplo: 
e o produto de dois vetores, sob a lei de multiplicação do grupo, está contido no espaço :

$$
\begin{aligned}
z=x \cdot y & =\left(\sum_{R} x_{R} R\right)\left(\sum_{S} y_{S} S\right)=\sum_{R, S} x_{R} y_{S} R S= \\
& =\sum_{T}\left(\sum_{S} x_{T S^{-1}} y_{S}\right) T=\sum_{T}\left(\sum_{S} x_{S} y_{S^{-1}} T\right) T= \\
& =\sum_{T} z_{T} T
\end{aligned}
$$

Definição 1 Um espaço vetorial o qual é fechado sobre uma regra de multiplicação define uma álgebra.

Assim o espaço do grupo, munido da lei de multiplicação do grupo, define o que chamamos de álgebra do grupo.

Qualquer representação da álgebra $A$ dá obviamente uma representação do grupo, pois se $s=\sum_{S} \alpha_{S} S$, então

$$
\langle x|s| y\rangle=\sum_{S} \alpha_{S}\langle x|S| y\rangle
$$

de modo que

$$
D(s)=\sum_{S} \alpha_{S} D(S)
$$

e vice-versa. Se uma destas representações é redutível (ou irredutivel) então a outra também será. A equação (4.29) nos dá a representação regular para a álgebra, a qual é totalmente redutível e contém cada representação irredutível um número de vezes igual à sua dimensão. de modo então que a representação do grupo também é totalmente redutível.

Por uma sub-álgebra $B$ de $A$, entendemos por um sub-espaço contido em $A$, o qual $e$ fechado sobre a mesma lei de multiplicação da álgebra $A$.

Definição 2 Se uma sub-álgebra $B$ tem a propriedade, para u em $B, s \cdot$ u está também em $B$ para qualquer elemento $s$ em $A$, tentão $B$ é um ideal à esquerda.

Assim sendo a álgebra $A$ pode ser decomposta na soma direta de seus ideais :

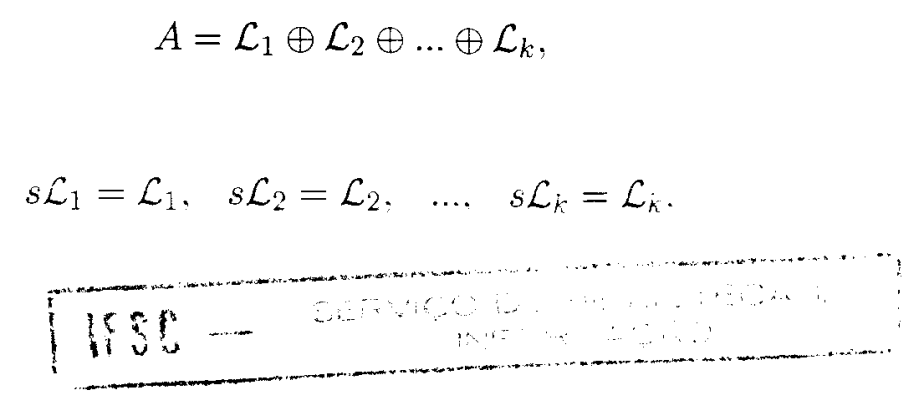


de modo que todo elemento de $A$ é unicamente expresso como a soma dos elementos de seus ideais :

$$
s=s_{1}+s_{2}+\ldots+s_{k}
$$

e portanto

$$
D(s)=D\left(s_{1}\right) \oplus D\left(s_{2}\right) \oplus \ldots \oplus D\left(s_{k}\right),
$$

reduzindo assim a representação da álgebra e consequentemente a do grupo.

A identidade $e$ do grupo $G$, está na álgebra $A$ de modo que :

$$
e \cdot s=s \cdot e
$$

e portanto podemos expressa-la como :

$$
e=e_{1}+e_{2}+. .+e_{k}
$$

onde $e_{i} \in \mathcal{L}_{i}$, e tem as seguintes propriedades :

$$
e_{i}^{2}=e_{i}, \quad e_{i} \cdot e_{j}=e_{j} \cdot e_{i}, \quad(i \neq j)
$$

como foi deduzido em [29].

Portanto, cada elemento $e_{i}$ é idenpotênte, isto é $e_{i}^{2}=e_{i}$, e é um gerador do ideal $\mathcal{L}_{i}$, já que $s \cdot e_{i} \in \mathcal{L}_{i}$.

Teorema $9 \mathrm{Se}$ um ideal $\mathcal{L}_{i}$ não contém ideais próprios (ideais não triviais), então este nos provem uma representação irredutível. Tal ideal é dito ser mínimo.

Portanto a idéia é simples : Tendo resolvido a identidade da forma :

$$
e=e_{1}+e_{2}+. .+e_{k}, \quad e_{i} \in \mathcal{L}_{i}
$$

onde cada $\mathcal{L}_{i}$ é um ideal mínimo, podemos para um dado $e_{i}$ gerar os outros elementos do ideal $\mathcal{L}_{i}$, sendo que cada um destes elementos nos dará um vetor da base que porta a representação da álgebra, e consequentemente do grupo.

\subsubsection{Operadores de Young}

Os operadores de Young são essencialmente os geradores idempotêntes dos ideais mínimos da álgebra, construida a partir dos grupos de permutação, e que resolvem a identidade da 
seguinte forma :

$$
e=y_{1}+y_{2}+. .+y_{k}
$$

nos dando as bases para as representações irredutíveis do grupo.

Contruimos os operadores de Young utilizando os Young tableaux, discutidos anteriormente, da maneira que se segue :

Para uma partição $[\lambda]$ do $\mathbf{S}_{n}$ desenhe o diagrama de Young. Coloque os números $1,2, \ldots, n$ de modo a formarmos um Young tableau. Uma vez fixado o tableau, consideremos dois tipos de permutações : as permutações horizontais $p$, as quais trocam os símbolos de uma uma mesma linha e as permutações verticais $q$, trocando somente os símbolos de uma mesma coluna. Construimos contruimos assim as seguintes quantidades:

$$
\begin{gathered}
P=\sum_{p} p \quad \text { "simetrizador". } \\
Q=\sum_{q} \delta_{q} q \quad \text { "anti-simetrizador", }
\end{gathered}
$$

onde $\delta_{q}$ é a paridade da permutação $q$.

Assim o operador de Young, definido por :

$$
Y=Q P
$$

o qual é essencialmente idempotênte e gera um ideal à esquerda mínimo, o qual nos provem uma representação irredutível do $\mathbf{S}_{n}$.

Teorema 10 As representações obtidas para diferentes diagramas são não equivalentes. Diferentes Young tableaux, com o mesmo diagrama, dão representações equivalentes.

Podemos ilustrar o problema para alguns casos simples:

- $n=2$

O operador de Young $Y_{1}=e+(12)$ corresponde ao tableau :

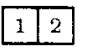

e o operador $Y_{2}=\epsilon-(12)$ corresponde à : 
\begin{tabular}{l|}
\hline \\
\hline 2
\end{tabular}

os quais são idempotêntes e resolvem a identidade :

$$
e=\frac{e+(12)}{2}+\frac{e+(12)}{2}
$$

de modo que o operador $Y_{1}$ nos gera :

$$
\begin{aligned}
e Y_{1} & =Y_{1} \\
(12) Y_{1} & =Y_{1}
\end{aligned}
$$

um ideal unidimensional que porta a representação simétrica. E o operador $Y_{2}$ nos dá :

$$
\begin{aligned}
e Y_{2} & =Y_{2} \\
(12) Y_{2} & =-Y_{2}
\end{aligned}
$$

a representação anti-simétrica (unidimensional).

- $\mathrm{n}=3$

O tableau :

\section{\begin{tabular}{|l|l|l|}
\hline 1 & 2 & 3 \\
\hline
\end{tabular}}

está associado ao seguinte operador :

$$
S=\sum_{R} R
$$

para a representação simétrica, e o tableau :

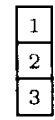

nos dá :

$$
A=\sum_{R} \delta_{R} R
$$

para a representaçāo anti-simétrica.

Agora para a partição [21] existem dois tableaux possiveis. Para o primeiro tableau teremos : 


$$
\sqrt{\frac{12}{3}} \rightarrow\left\{\begin{array}{l}
P=e+(12) \\
Q=e-(13)
\end{array}\right.
$$

e portanto:

$$
Y=Q P=e+(12)-(13)-(123)
$$

Para o segundo tableau :

$$
\begin{aligned}
& \frac{1}{2} 3^{3} \\
& Q^{\prime}=e-(12)
\end{aligned}
$$

e portanto :

$$
Y^{\prime}=Q^{\prime} P^{\prime}=e-(12)+(13)-(132)
$$

de modo a obtermos a seguinte resolução para a identidade :

$$
e=S+A+\frac{Y}{3}+\frac{Y^{\prime}}{3}
$$

Os geradores $S$ e $A$ nos dão dois ideais unidimensionais, que portam, respectivamente a representação simétrica e anti-simétrica. Já para $Y=Y_{1}$ temos :

$$
\begin{array}{rlrl}
e Y_{1} & =Y_{1}, & & (23) Y_{1}=Y_{1}-Y_{2}, \\
(12) Y_{1} & =Y_{2}, & & (123) Y_{1}=Y_{2}-Y_{1}, \\
(13) Y_{1} & =-Y_{1}, & (132) Y_{1}=-Y_{2},
\end{array}
$$

sendo :

$$
\begin{aligned}
& Y_{1}=e+(12)+(13)-(123), \\
& Y_{2}=e+(12)-(23)-(132),
\end{aligned}
$$

os dois elementos de base do ideal $\mathcal{L}_{Y}$, bidimensional, portando a representação mista do $\mathbf{S}_{3}$. tendo :

$$
\begin{aligned}
& \varphi_{1}=Y_{1} \Phi \\
& \varphi_{2}=Y_{2} \Phi
\end{aligned}
$$

como base. 
Equivalentemente, para $Y^{\prime}=Y_{1}^{\prime}$

$$
\begin{array}{rlrl}
e Y_{1}^{\prime} & =Y_{1}^{\prime}, & (23) Y_{1}^{\prime}=Y_{1}^{\prime}-Y_{2}^{\prime}, \\
(12) Y_{1}^{\prime} & =-Y_{1}^{\prime}, & (123) Y_{1}^{\prime}=-Y_{2}^{\prime}, \\
(13) Y_{1}^{\prime}=Y_{2}^{\prime}, & (132) Y_{1}^{\prime}=Y_{2}^{\prime}-Y_{1}^{\prime},
\end{array}
$$

com :

$$
\begin{aligned}
& Y_{1}^{\prime}=e-(12)+(13)-(132) \\
& Y_{2}^{\prime}=e+(13)-(23)-(123)
\end{aligned}
$$

sendo os dois elementos de base para o ideal $\mathcal{L}_{Y^{\prime}}$, portado uma representação bidimensional do $\mathbf{S}_{3}$, equivalente à aquela gerada por $\mathcal{L}_{Y}$, e com a base sendo :

$$
\begin{aligned}
& \varphi_{1}^{\prime}=Y_{1}^{\prime} \Phi \\
& \varphi_{2}^{\prime}=Y_{2}^{\prime} \Phi .
\end{aligned}
$$

- $\mathrm{n}=4$

As partições [4] e [1 $\left.{ }^{4}\right]$ dão, respectivamente, as representações simétrica e anti-simétrica. Para o diagrama :

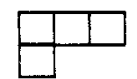

teremos :

$$
\begin{array}{|l|l}
\hline 1 & 2 \\
\hline 4 & 3
\end{array} \rightarrow\left\{\begin{array}{c}
P_{1}=e+(12)+(23)+(13)+(123)+(132) \\
Q_{1}=e-(14)
\end{array}\right.
$$

sendo $Y_{1}=Q_{1} P_{1}$,

$$
\begin{array}{|c|c|c}
\hline 1 & 2 & 4 \\
\hline 3 &
\end{array} \rightarrow\left\{\begin{array}{c}
P_{2}=e+(12)+(24)+(14)+(124)+(142) \\
Q_{2}=e-(13)
\end{array}\right.
$$

sendo $Y_{2}=Q_{2} P_{2}$

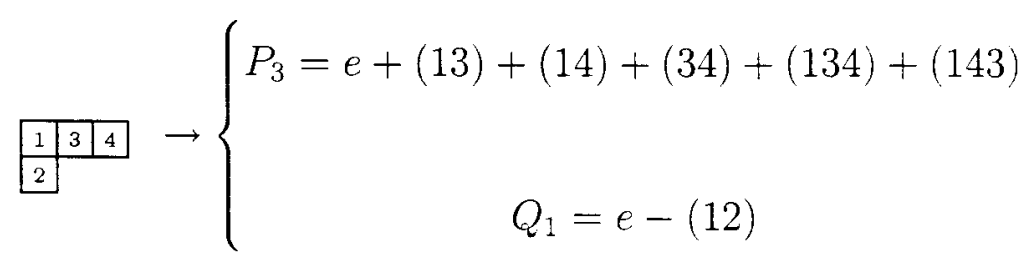

sendo $Y_{3}=Q_{3} P_{3}$. Para o diagrama : 
teremos :

$$
\begin{array}{|l|l}
\hline 1 & 2 \\
\hline 3 & 4 \\
\hline
\end{array} \rightarrow\left\{\begin{array}{l}
P_{4}=e+(12)+(34)+(12)(34) \\
Q_{4}=e-(13)-(24)+(13)(24)
\end{array}\right.
$$

sendo $Y_{4}=Q_{4} P_{4}$

$$
\begin{array}{|l|l}
\hline \frac{3}{2} & 3 \\
\hline & 4
\end{array} \rightarrow\left\{\begin{array}{l}
P_{5}=e+(12)+(34)+(12)(34) \\
Q_{5}=e-(13)-(24)+(13)(24)
\end{array}\right.
$$

sendo $Y_{5}=Q_{5} P_{5}$. E assim por diante.

\subsection{Operadores de Projeção}

Este método também busca a construção das bases irredutíveis para as irreps do grupo de permutação, a partir de uma função arbitrária no espaço do grupo.

\subsubsection{Definição}

A partir de uma função $\phi$, arbitrária, podemos obter uma representação aplicando as transformações do grupo. No caso mais geral ( $\phi$ completamente sem simetria) cada função $R o$ será linearmente independente de modo a obtermos uma base para a representação regular. conforme discutido anteriormente. Estamos interessados em reduzir a representação em suas componentes irredutíveis, isto é, construir as bases que portam as representações irredutiveis do grupo. Isto é feito através dos operadores de projeção definidos por :

$$
\rho_{\mu \nu}^{(j)}=\frac{n_{j}}{g} \sum_{R} D_{\mu \nu}^{(j)^{*}}(R) R
$$

onde $g$ é a ordem do grupo, $n_{j}$ é a dimensão da irrep (unitária) $D_{\mu \nu}^{(j)}$, com $\mu, \nu=1,2, \ldots, n_{j}$. e a soma sendo feita sob todos os elementos do grupo. Uma demonstração é feita em [28: Denotamos aqui os operadores isomorfos ao grupo pela a mesma notação dos elementos dic: 


\subsection{OPERADORES DE PROJEÇÃO}

grupo. A determinação dos operadores de projeção requer o conhecimento de dodas as irreps não equivalentes do grupo. Os operadores de projeção estão definidos sob um espaço vetorial dotado de um produto escalar conveniente, de modo a obtermos um representação unitária.

Portanto, construimos a base para a representação irredutível aplicando o operador de projeção numa função $\phi$ :

$$
\varphi_{\mu \nu}^{(j)}=\rho_{\mu \nu}^{(j)} \phi
$$

pois :

$$
\begin{aligned}
R \varphi_{\mu \nu}^{(j)} & =\frac{n_{j}}{g} \sum_{S} D_{\mu \nu}^{(j)^{*}}(S) R S \phi=\frac{n_{j}}{g} \sum_{T} D_{\mu \nu}^{(j)^{*}}\left(R^{-1} T\right) T \phi= \\
& =\frac{n_{j}}{g} \sum_{T}\left(\sum_{l} D_{\mu l}^{(j)^{*}}\left(R^{-1}\right) D_{l \nu}^{(j)^{*}}(T)\right) T \phi \\
& =\sum_{l} D_{\mu l}^{(j)}{ }^{-1 *}(R)\left(\frac{n_{j}}{g} \sum_{T} D_{l \nu}^{(j)^{*}}(T) T \phi\right)
\end{aligned}
$$

e portanto :

$$
R \varphi_{\mu \nu}^{(j)}=\sum_{l=1}^{n_{j}} D_{l \mu}^{(j)}(R) \varphi_{l \nu}^{(j)}
$$

Vemos, através de (4.66), que o operador de projeção $\rho_{\mu \nu}^{(j)}$ nos dá $n_{j}$ bases de dimensão $n_{j}$, uma para cada valor de $\nu$. Podemos dizer então que os operadores de projeção pertencentes a uma mesma "coluna" nos dão uma base, sendo denominados de partners.

Podemos calcular o produto de operadores de projeção. sob a lei de multiplicação do grupo :

$$
\begin{aligned}
\rho_{m \mu}^{(i)} \rho_{\nu n}^{(j)} & =\frac{n_{i} n_{j}}{g^{2}} \sum_{R, S} D_{m \mu}^{(i) *}(R) D_{\nu n}^{(j)^{*}}(S) R S \\
& =\frac{n_{i} n_{j}}{g^{2}} \sum_{R, T} D_{m \mu}^{(i)}{ }^{*}(R)\left(\sum_{l}{D_{\nu l}^{(j)}}^{*}\left(R^{-1}\right) D_{l n}^{(j)^{*}}(T)\right) T \\
& =\frac{n_{i} n_{j}}{g^{2}} \sum_{R, T, l} D_{m \mu}^{(i)}{ }^{*}(R) D_{l \nu}^{(j)}(R) D_{l n}^{(j)^{*}}(T) T \\
& =\frac{n_{i} n_{j}}{g^{2}} \sum_{T, l}\left(\sum_{R} D_{l \nu}^{(j)}(R) D_{m \mu}^{(i)}{ }^{*}(R)\right) D_{l n}^{(j)^{*}}(T) T
\end{aligned}
$$

onde lançamos mão da relação de ortogonalidade para as representações unitárias, [29] :

$$
\sum_{R} D_{i l}^{(\mu)}(R) D_{j m}^{(\nu) *}(R)=\frac{g}{n_{\mu}} \delta_{\mu \nu} \delta_{i j} \varepsilon_{i m}
$$


de modo que:

$$
\begin{aligned}
\rho_{m \mu}^{(i)} \rho_{\nu n}^{(j)} & =\frac{n_{i} n_{j}}{g^{2}} \sum_{T, l}\left(\frac{g}{n_{i}} \delta_{l m} \delta_{i j} \delta_{\mu \nu}\right) D_{l n}^{(j)^{*}}(T) T= \\
& =\left(\frac{n_{j}}{g} \sum_{T}{D_{m n}^{(j)}}^{*}(T) T\right) \delta_{i j} \delta_{\mu \nu}
\end{aligned}
$$

e portanto deduzindo uma importante relação para os operadores de projeção :

$$
\rho_{m \mu}^{(i)} \rho_{\nu n}^{(j)}=\delta_{i j} \delta_{\mu \nu} \rho_{m n}^{(j)}
$$

Ainda temos que :

$$
\begin{aligned}
\left(\rho_{\mu \nu}^{(j)}\right)^{\dagger} & =\frac{n_{j}}{g} \sum_{R} D_{\mu \nu}^{(j)}(R) R^{\dagger}= \\
& =\frac{n_{j}}{g} \sum_{R} D_{\mu \nu}^{(j)}(R) R^{-1}= \\
& =\frac{n_{j}}{g} \sum_{S} D_{\mu \nu}^{(j)}\left(S^{-1}\right) S= \\
& =\frac{n_{j}}{g} \sum_{S} D_{\nu \mu}^{(j)}(S) S,
\end{aligned}
$$

e portanto :

$$
\left(\rho_{\mu \nu}^{(j)}\right)^{\dagger}=\rho_{\nu \mu}^{(j)}
$$

Deste modo teremos que :

$$
\begin{aligned}
\left\langle\varphi_{\mu \nu}^{(i)} \mid \psi_{\mu^{\prime} \nu^{\prime}}^{(j)}\right\rangle & =\left\langle\rho_{\mu \nu}^{(i)} \phi \mid \rho_{\mu^{\prime} \nu^{\prime}}^{(j)} \phi\right\rangle=\left\langle\phi \mid \rho_{\nu \mu}^{(i)} \rho_{\mu^{\prime} \nu^{\prime}}^{(j)} \phi\right\rangle= \\
& =\delta_{i j} \delta_{\mu \mu^{\prime}}\left\langle\phi \mid \rho_{\nu \nu^{\prime}}^{(j)} \phi\right\rangle=\delta_{i j} \delta_{\mu \mu^{\prime}}\left\langle o \mid \rho_{\nu \mu}^{(j)} \rho_{\mu \nu^{\prime}}^{(j)} O\right\rangle= \\
& =\delta_{i j} \delta_{\mu \mu^{\prime}}\left\langle\rho_{\mu \nu}^{(i)} \phi \mid \rho_{\mu \nu^{\prime}}^{(j)} \phi\right\rangle .
\end{aligned}
$$

e em particular se $\nu=\nu^{\prime}$ e $i=j$ :

$$
\left\langle\varphi_{\mu \nu}^{(j)} \mid \varphi_{\mu^{\prime} \nu}^{(j)}\right\rangle \simeq \delta_{\mu \mu^{\prime}}
$$

mostrando portanto que as bases geradas pelos operadores de projeção são ortogonais. ( que era de se esperar já que os operadores $R$ e as representações são unitários. No entanto () operador de projeção aplicado a um conjunto de funções ortogonais destroi a ortogonalidade das funções originais. o qual é o caso da aplicação dos operadores de projeção do grupo $\mathbf{S}$ nos hiperesféricos do $S O(9)$. sendo então necessário impor a ortogonalidade a estas funçôe. 


\subsubsection{Bases Irredutíveis para o $\mathrm{S}_{3}$}

Faremos uso das representações irredutíveis de acordo com [29], para assim contruirmos os operadores de projeção que nos darão as bases irredutíveis.

- Representação simétrica, unidimensional :

Na representação Simétrica tem-se $D(R)=1$ para todos os elementos do grupo, de forma a termos o seguinte operador de projeção :

$$
\rho^{(s)}=\frac{1}{6}\left[e+(12)+(13)+(23)+(123)+(132)_{i}^{\prime},\right.
$$

de modo que tenhamos como base :

$$
\begin{aligned}
\varphi^{(s)} & =\rho^{(s)} \phi\left(r_{1}, r_{2}, r_{3}\right)= \\
& =\frac{1}{6}\left[\phi\left(r_{1}, r_{2}, r_{3}\right)+\phi\left(r_{2}, r_{1}, r_{3}\right)+\phi\left(r_{3}, r_{2}, r_{1}\right)+\right. \\
& \left.+\phi\left(r_{1}, r_{3}, r_{2}\right)+\phi\left(r_{2}, r_{3}, r_{1}\right)+\phi\left(r_{3}, r_{1}, r_{2}\right)\right] .
\end{aligned}
$$

- Representação anti- simétrica :

Para a representação Anti-Simétrica tem-se $D(R)=1$ para todas as permutações pares, $R \equiv\{(123),(132)\}$, e $D(R)=-1$ para as permutações ímpares, $R \equiv\{(12),(13)$, (23)\}, nos dando portanto :

$$
\rho^{(a)}=\frac{1}{6}[e-(12)-(13)-(23)+(123)+(132)],
$$

de modo que :

$$
\begin{aligned}
\varphi^{(a)} & =\rho^{(a)} \phi\left(r_{1}, r_{2}, r_{3}\right)= \\
& =\frac{1}{6}\left[\phi\left(r_{1}, r_{2}, r_{3}\right)-\phi\left(r_{2}, r_{1}, r_{3}\right)-\phi\left(r_{3}, r_{2}, r_{1}\right)-\right. \\
& \left.-\phi\left(r_{1}, r_{3}, r_{2}\right)+\phi\left(r_{2}, r_{3}, r_{1}\right)+\phi\left(r_{3}, r_{1}, r_{2}\right)\right]
\end{aligned}
$$

- Representação Mista : 
$\mathrm{Na}$ construção das bases para a representação mista, escolhemos a seguinte representação matricial (unitária), [29] :

$$
\begin{array}{ll}
D(e)=\left(\begin{array}{ll}
1 & 0 \\
0 & 1
\end{array}\right) & D(12)=\left(\begin{array}{cc}
1 & 0 \\
0 & -1
\end{array}\right) \\
D(13)=\left(\begin{array}{cc}
-1 / 2 & -\sqrt{3} / 2 \\
-\sqrt{3} / 2 & 1 / 2
\end{array}\right) & D(23)=\left(\begin{array}{cc}
-1 / 2 & \sqrt{3} / 2 \\
\sqrt{3} / 2 & 1 / 2
\end{array}\right) \\
D(123)=\left(\begin{array}{cc}
-1 / 2 & \sqrt{3} / 2 \\
-\sqrt{3} / 2 & -1 / 2
\end{array}\right) & D(132)=\left(\begin{array}{cc}
-1 / 2 & -\sqrt{3} / 2 \\
\sqrt{3} / 2 & -1 / 2
\end{array}\right)
\end{array}
$$

de modo a que tenhamos:

$$
\begin{aligned}
& \rho_{11}^{(m)}=\frac{1}{3}\left[e+(12)-\left(\frac{(13)+(23)+(123)+(132)}{2}\right)\right], \\
& \rho_{21}^{(m)}=\frac{\sqrt{3}}{6}[(23)-(13)-(123)+(132)], \\
& \rho_{12}^{(m)}=\frac{\sqrt{3}}{6}[(23)-(13)+(123)-(132)], \\
& \rho_{22}^{(m)}=\frac{1}{3}\left[e-(12)+\left(\frac{(13)+(23)-(123)-(132)}{2}\right)\right],
\end{aligned}
$$

e portanto :

$$
\begin{aligned}
\varphi_{11}^{(m)} & =\rho_{11}^{(m)} \phi\left(r_{1}, r_{2}, r_{3}\right)= \\
& =\frac{1}{6}\left\{2 \phi\left(r_{1}, r_{2}, r_{3}\right)+2 \phi\left(r_{2}, r_{1}, r_{3}\right)-\phi\left(r_{1}, r_{3}, r_{2}\right)-\phi\left(r_{3}, r_{2}, r_{1}\right)-\right. \\
& \left.-\phi\left(r_{2}, r_{3}, r_{1}\right)+-\phi\left(r_{3}, r_{1}, r_{2}\right)\right\} \\
\varphi_{21}^{(m)} & =\rho_{21}^{(m)} \phi\left(r_{1}, r_{2}, r_{3}\right)= \\
& =\frac{\sqrt{3}}{6}\left\{\phi\left(r_{1}, r_{3}, r_{2}\right)-\phi\left(r_{3}, r_{2}, r_{1}\right)-\phi\left(r_{2}, r_{3}, r_{1}\right)+\phi\left(r_{3}, r_{1}, r_{2}\right)\right\} \\
\varphi_{12}^{(m)} & =\rho_{12}^{(m)} \phi\left(r_{1}, r_{2}, r_{3}\right)= \\
& =\frac{\sqrt{3}}{6}\left\{\phi\left(r_{1}, r_{3}, r_{2}\right)-\phi\left(r_{3}, r_{2}, r_{1}\right)+\phi\left(r_{2}, r_{3}, r_{1}\right)-\phi\left(r_{3}, r_{1}, r_{2}\right)\right\} \\
& =\frac{1}{6}\left\{2 \phi\left(r_{1}, r_{2}, r_{3}\right)-2 \phi\left(r_{2}, r_{1}, r_{3}\right)+\phi\left(r_{1}, r_{3}, r_{2}\right)+\phi\left(r_{3}, r_{2}, r_{1}\right)-\right. \\
\varphi_{22}^{(m)} & \rho_{22}^{(m)} \phi\left(r_{1}, r_{2}, r_{3}\right)= \\
& =\frac{1}{(m)},
\end{aligned}
$$


- $\left.\phi\left(r_{2}, r_{3}, r_{1}\right)-\phi\left(r_{3}, r_{1}, r_{2}\right)\right\}$,

onde cada par $\left\{\varphi_{11}^{(m)}, \varphi_{21}^{(m)}\right\}$ e $\left\{\varphi_{12}^{(m)}, \varphi_{22}^{(m)}\right\}$, os quais são correspondentes aos pares de partners $\left\{\rho_{11}^{(m)}, \rho_{21}^{(m)}\right\}$ e $\left\{\rho_{12}^{(m)}, \rho_{22}^{(m)}\right\}$ respectivamente, carrega a mesma irrep mista do $\mathbf{S}_{\mathbf{3}}$.

\subsubsection{Operadores de Projeção e Álgebra do Grupo}

A partir da seguinte relação:

$$
\sum_{j=1}^{N} \sum_{m, k=1}^{n_{j}} \frac{n_{j}}{g} D_{m k}^{j}(R) D_{m k}^{j}(S)=\delta_{R S}
$$

de [28], pag.85, onde $N$ é o número de irreps não equivalentes, podemos determinar a inversa do operador de projeção :

$$
R=\sum_{j=1}^{N} \sum_{m=1}^{n_{j}} \sum_{k=1}^{n_{j}} D_{m k}^{j}(R) \rho_{m k}^{(j)}
$$

Em particular se fizermos $R=e$, teremos :

$$
e=\sum_{j=1}^{N} \sum_{m=1}^{n_{j}} \rho_{m m}^{(j)},
$$

onde o operador $\rho_{m m}^{(j)}$, de acordo com :

$$
\rho_{m \mu}^{(i)} \rho_{\nu n}^{(j)}=\delta_{i j} \delta_{\mu \nu} \rho_{m n}^{(j)}
$$

é um operador idempotênte, isto é :

$$
\left(\rho_{m m}^{(j)}\right)^{2}=\rho_{m m}^{(j)}
$$

Se considerarmos a álgebra do grupo sendo formada pelos $\sum_{j=1}^{N} n_{j}^{2}$ vetores $\rho_{n m}^{(j)}$, de acordo com

$$
\begin{gathered}
\rho_{\alpha \beta}^{(i)} \rho_{n m}^{(j)}=\delta_{i j} \delta_{\beta n} \rho_{\alpha m}^{(j)}, \\
\rho_{\alpha \beta}^{(i)} \rho_{m m}^{(j)}=\delta_{i j} \delta_{\beta m} \rho_{\alpha m}^{(j)},
\end{gathered}
$$

temos que os operadores de projeção de uma determinada irrep $(j)$, pertencentes a uma mesma coluna (partners) $m$, formam um ideal à esquerda $\mathcal{L}_{m}^{(j)}, n_{j}$-dimensional, de acordo com (4.87) :

$$
\rho_{\alpha \beta}^{(i)} \mathcal{L}_{m}^{(j)}=\delta i j \mathcal{L}_{m}^{(j)} .
$$




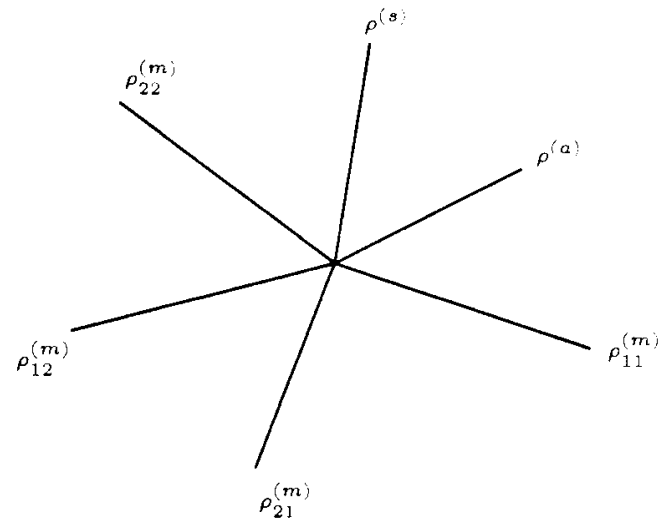

Figura 4.2: Álgebra dos Operadores de Projeção do Grupo $\mathbf{S}_{3}$.

tendo como gerador o operador $\rho_{m m}^{(j)}$, equação (4.88). Numa abordagem também feita em $[22]$

Portanto, a álgebra formada pelos operadores de projeção é decomposta em seus ideais :

$$
\mathcal{L}=\sum_{j=1}^{N} \sum_{m=1}^{n_{j}} \mathcal{L}_{m}^{(j)}
$$

onde cada ideal é um ideal mínimo, formado pelos partners, os quais portam a representação irredutível, decompondo a identidade nos geradores idempotêntes :

$$
e=\sum_{j=1}^{N} \sum_{m=1}^{n_{j}} \rho_{m m}^{(j)} .
$$

Assim sendo, vemos que os operadores de projeção são análogos aos operadores de Young. discutidos anteriormente, relacionados através de uma mudança de base. Na construção dos operadores de Young não foi exigido o conhecimento das representações do grupo, porém. como veremos, as representações por eles obtidas não são unitárias.

Podemos deixar clara a relação entre os operadores de projeção e os operadores de Young através de um exemplo :

De (4.52), vemos que os operadores de Young, $Y_{1}$ e $Y_{2}$, para o tableau :

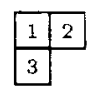

nos dão a seguinte representação para o $\mathbf{S}_{3}$ : 


$$
\begin{array}{ll}
D^{\prime}(e)=\left(\begin{array}{ll}
1 & 0 \\
0 & 1
\end{array}\right) & D^{\prime}(12)=\left(\begin{array}{ll}
0 & 1 \\
1 & 0
\end{array}\right) \\
D^{\prime}(13)=\left(\begin{array}{cc}
-1 & -1 \\
0 & 1
\end{array}\right) & D^{\prime}(23)=\left(\begin{array}{cc}
1 & 0 \\
-1 & -1
\end{array}\right) \\
D^{\prime}(123)=\left(\begin{array}{cc}
0 & 1 \\
-1 & -1
\end{array}\right) & D^{\prime}(132)=\left(\begin{array}{cc}
-1 & -1 \\
1 & 0
\end{array}\right)
\end{array}
$$

as quais não são unitárias. Podemos levar estas representações às representações de [29] através da transformação de similaridade :

$$
S D^{\prime}(R) S^{-1}=D(R),
$$

onde no nosso caso :

$$
S=\left(\begin{array}{cc}
\sqrt{3} / 6 & \sqrt{3} / 6 \\
1 / 2 & -1 / 2
\end{array}\right)
$$

Assim sendo :

$$
\left(\begin{array}{c}
\tilde{Y}_{1} \\
\tilde{Y}_{2}
\end{array}\right)\left(\begin{array}{cc}
\sqrt{3} / 6 & 1 / 2 \\
\sqrt{3} / 6 & -1 / 2
\end{array}\right)\left(\begin{array}{c}
Y_{1} \\
Y_{2}
\end{array}\right)
$$

onde teremos:

$$
\begin{gathered}
\tilde{Y}_{1}=\frac{\sqrt{3}}{6}(2 e+2(12)-(13)-(23)-(123)-(132)), \\
\tilde{Y_{2}}=\frac{1}{2}((23)+(13)-(123)-(132)),
\end{gathered}
$$

de modo que se compararmos com os operadores de projeção do $\mathbf{S}_{3}$, equação (4.80), teremos

$$
\begin{gathered}
\tilde{Y}_{1}=2 \sqrt{3} \rho_{11}^{(m)}, \\
\tilde{Y}_{2}=\sqrt{3} \rho_{21}^{(m)},
\end{gathered}
$$

mostrando que os operadores de Young e os de projeção estão relacionados através de uma mudança de base. 


\subsection{Produto de Kronecker de Irreps}

O produto de Kronecker de duas representações irredutíveis exerce uma lei fundamental na teoria de sistemas acoplados. Para os grupos de permutação o produto de Kronecker é usualmente chamado de produto interno, já que este consiste no produto de duas irreps que se referem às mesmas partículas.

Iremos nesta seção primeiramente discutir a decomposição do produto de Kronecker em suas componentes irredutíveis, séries de Clebsch-Gordan. Posteriormente iremos esboçar suscintamente o problema de se determinar as bases para as representações contidas no produto de Kronecker, Coeficientes de Clebsch-Gordan.

\subsubsection{Séries de Clebsch-Gordan}

Supondo que $\psi_{m_{1}}^{\left(\nu_{1}\right)}\left(m_{1}=1,2, \ldots, n_{\nu_{1}}\right)$ e $\varphi_{m_{2}}^{\left(\nu_{2}\right)}\left(m_{2}=1,2, \ldots, n_{\nu_{2}}\right)$ portam, respectivamente as irreps $\left[\nu_{1}\right]$ e $\left[\nu_{2}\right]$ do grupo $G$. Os $\nu_{1} \cdot \nu_{2}$ produtos :

$$
\left|m_{1} m_{2}\right\rangle \equiv \psi_{m_{1}}^{\left(\nu_{1}\right)} \varphi_{m_{2}}^{\left(\nu_{2}\right)}
$$

formam um espaço para as representações de $G$. Portanto sendo :

$$
\begin{aligned}
R\left|m_{1} m_{2}\right\rangle & =\sum_{m_{1}^{\prime} m_{2}^{\prime}} D_{m_{1}^{\prime} m_{1}}^{\nu_{1}}(R) D_{m_{2}^{\prime} m_{2}}^{\nu_{2}}(R)\left|m_{1}^{\prime} m_{2}^{\prime}\right\rangle= \\
& =\sum_{m_{1}^{\prime} m_{2}^{\prime}} D_{m_{1}^{\prime} m_{2}^{\prime} ; m_{1} m_{2}}^{\nu_{1} \times \nu_{2}}(R)\left|m_{1}^{\prime} m_{2}^{\prime}\right\rangle
\end{aligned}
$$

com :

$$
D_{m_{1}^{\prime} m_{2}^{\prime} ; m_{1} m_{2}}^{\left(\nu_{1} \times \nu_{2}\right)}(R)=D_{m_{1}^{\prime} m_{1}}^{\nu_{1}}(R) D_{m_{2}^{\prime} m_{2}}^{\nu_{2}}(R)
$$

isto é :

$$
D^{\left(\nu_{1} \times \nu_{2}\right)}(R)=D^{\nu_{1}}(R) \times D^{\nu_{2}}(R)
$$

geramos uma representação, a qual é chamada de produto de Kronecker das irreps $\left[\nu_{1}\right]$ e $\left[\nu_{2}\right.$ denotando o produto por $\left[\nu_{1}\right] \times\left[\nu_{2}\right]$, o qual é geralmente redutível. O produto de Kronecker pode ser reduzido em suas componetes irredutíveis da seguinte forma : 


$$
\left[\nu_{1}\right] \times\left[\nu_{2}\right]=\sum_{\nu}\left(\nu_{1} \nu_{2} \nu\right)[\nu]
$$

onde $\left(\nu_{1} \nu_{2} \nu\right)$ é o número de vezes que a irrep $[\nu]$ aparece, sendo dado em função dos caracters simples, [28] :

$$
\left(\nu_{1} \nu_{2} \nu\right)=\sum_{i} \frac{q_{i}}{g} \chi_{i}^{\left(\nu_{1}\right)} \chi_{i}^{\left(\nu_{2}\right)} \chi_{i}^{(\nu)}
$$

onde $g_{i}$ é o número de elementos pertencentes à classe $i$ e $g$ a ordem do grupo. De (4.102) fica claro que :

$$
\left(\nu_{1} \nu_{2} \nu_{3}\right)=\left(\nu_{i} \nu_{j} \nu_{k}\right)
$$

onde $(i, j, k)$ é qualquer permutação de $(1,2,3)$, de modo que o produto de Kronecker independa da ordem que é feito. A equação (4.101) é referida como série de Clebsch-Gordan.

Podemos então calcular as séries de Clebsch-Gordan a partir da tabela de caracters do grupo. Segue abaixo alguns resultados, na notação de diagramas, para as irreps do grupo $\mathbf{S}_{3}$, utilizando a tabela (4.1), dos caracters :

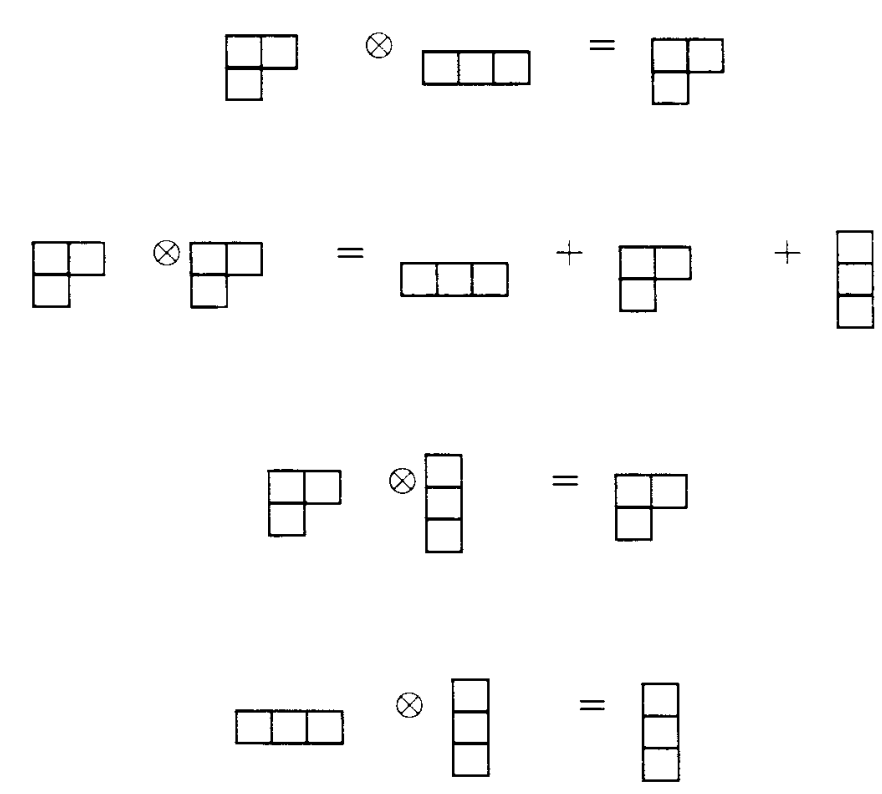

Podemos obter resultados para os grupos $\mathbf{S}_{4}$ e $\mathbf{S}_{5}$ a a partir das tabelas abaixo, de [28] : 


\begin{tabular}{l|lll} 
& {$[3]$} & {$[2,1]$} & {$\left[1^{3}\right]$} \\
\hline$[2,1] \times[2,1]$ & 1 & 1 & 1
\end{tabular}

Tabela 4.2: Séries de Clebsch-Gordan para o grupo $\mathbf{S}_{\mathbf{3}}$.

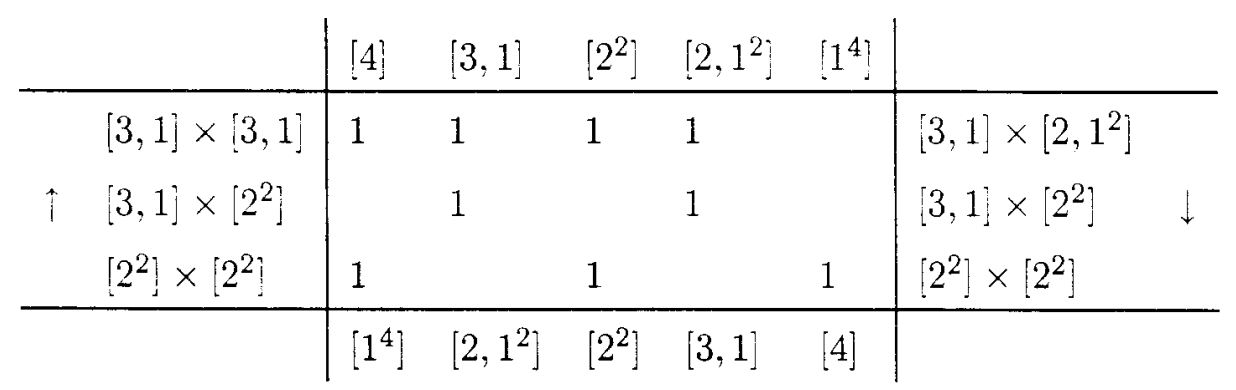

Tabela 4.3: Séries de Clebsch-Gordan para o grupo $\mathbf{S}_{\mathbf{4}}$.

\begin{tabular}{|c|c|c|c|c|c|c|c|c|c|c|}
\hline & & {$[5]$} & {$[4,1]$} & {$[3,2]$} & {$\left[3,1^{2}\right]$} & {$\left[2^{2}, 1\right]$} & {$\left[2,1^{3}\right]$} & {$\left[1^{5}\right]$} & & \\
\hline \multirow{7}{*}{$\uparrow$} & {$[4,1] \times[4,1]$} & 1 & 1 & 1 & 1 & & & & {$[4,1]\left[2,1^{3}\right],\left[2,1^{3}\right] \times[4,1]$} & \\
\hline & {$[4,1] \times[3,2]$} & & 1 & 1 & 1 & 1 & & & {$\left[4,1^{2} \times\left[2^{2}, 1\right],\left[2,1^{3}\right] \times[3,2]\right.$} & \\
\hline & {$[4,1] \times\left[3,1^{2}\right]$} & & 1 & 1 & 1 & 1 & 1 & & {$[4,1] \times\left[3,1^{2}\right],\left[2,1^{3}\right] \times\left\{3,1^{2}\right\}$} & \\
\hline & {$[3,2] \times[3,2]$} & 1 & 1 & 1 & 1 & 1 & 1 & & {$[3,2] \times\left[2^{2}, 1\right],\left[2^{2}, 1\right] \times[3,2$} & $i$ \\
\hline & {$[3,2] \times\left[3,1^{2}\right]$} & & 1 & 1 & 2 & 1 & 1 & & {$[3,2] \times\left[3,1^{2}\right],\left[2^{2}, 1\right] \times\left\{3,1^{2}\right\}$} & \\
\hline & {$\left[3,1^{2}\right] \times\left[3,1^{2}\right]$} & 1 & 1 & 2 & 1 & 2 & 1 & 1 & {$\left[3,1^{2}\right\} \times\left[3,1^{2}\right],\left[3,1^{2}\right] \times\left[3,1^{2}\right]$} & \\
\hline & & {$\left[1^{5}\right]$} & {$\left[2,1^{3}\right]$} & $\left\{2^{2}, 1\right\}$ & {$\left[3,1^{2}\right]$} & {$[3,2]$} & $\mid 4,1]$ & {$[5]$} & & \\
\hline
\end{tabular}

Tabela 4.4: Séries de Clebsch-Gordan para o grupo $\mathbf{S}_{\mathbf{5}}$.

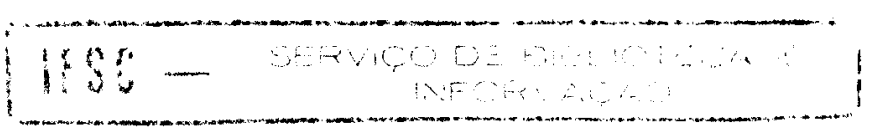


As tabelas dão os coeficientes na expanção do produto de Kronecker. Para os produtos do lado esquerdo os coeficientes se referem às partições no topo da tabela, e para os produtos à direita os coeficientes se referem às partição da parte de baixo da tabela.

\subsubsection{Coeficientes de Clebsch-Gordan}

Na seção anterior discutimos o problema de se determinar as irreps contidas no produto Kronecker. O produto de duas representações é, em geral, redutível. O problema que aqui nos é colocado é o de se encontrar as bases das irreps contidas no produto.

Nos é dado $n_{\nu_{1}}$ funções de base $\psi_{m_{1}}^{\left(\nu_{1}\right)}\left(m_{1}=1,2, \ldots, n_{\nu_{1}}\right)$ para a representação irredutível $\left[\nu_{1}\right]$ e $n_{\nu_{2}}$ funções de base $\varphi_{m_{2}}^{\left(\nu_{2}\right)}\left(m_{2}=1,2, \ldots, n_{\nu_{2}}\right)$ para a representação irredutivel $\left[\nu_{2}\right]$. Queremos encontrar um conjunto de $n_{\nu_{1}} \cdot n_{\nu_{2}}$ funções $\Psi_{m}^{(\nu)}\left(m=1,2, \ldots, n_{\nu_{1}} n_{\nu_{2}}\right)$, as quais são combinação linear dos produtos $\psi_{m_{1}}^{\left(\nu_{1}\right)} \varphi_{m_{2}}^{\left(\nu_{2}\right)}$ e que formam uma base para a irrep $[\nu]$. Através da seção anterior, sabemos que este conjunto existe somente se $[\nu]$ está contida em $\left[\nu_{1}\right] \times\left[\nu_{2}\right]$, isto é, somente se $\left(\nu_{1} \nu_{2} \nu\right) \neq 0$. Por outro lado, se $\left(\nu_{1} \nu_{2} \nu\right) \geq 1$ podemos formar diversos conjuntos de partners $\Psi_{m}^{(\nu)}$. Existirão de fato $\left(\nu_{1} \nu_{2} \nu\right)$ combinações lineares para uma mesma irrep. Para distiguir estes direfentes conjuntos usaremos a notação $\Psi_{m}^{(\nu), \tau}, \operatorname{com} m=1,2, \ldots, n_{\nu_{1}} n_{\nu_{2}}$ e $\tau=1,2, \ldots,\left(\nu_{1} \nu_{2} \nu\right)$. As funções $\Psi_{m}^{(\nu), \tau}$ são dadas por, [28] :

$$
\Psi_{m}^{(\nu), \tau}=\sum_{m_{1} m_{2}} C_{\nu_{1} m_{1}, \nu_{2} m_{2}}^{(\nu), \tau m} \psi_{m_{1}}^{\left(\nu_{1}\right)} \varphi_{m_{2}}^{\left(\nu_{2}\right)}
$$

onde $\tau$ é portanto o rótulo da multiplicidade e os $C_{\nu_{1}, m_{1}, \nu_{2} m_{2}}^{(\nu), \tau m}$ são os Coeficientes de ClebschGordan. Portanto a determinação dos Coeficientes de Clebsch-Gordan. [28] e [29], resolve por completo o problema de se determinar as bases irredutiveis contidas no produto de Kronecker.

Foge de nossa intenção estudar aqui esta teoria a fundo. Mostramos a seguir tabelas para os coefientes de Clebsch-Gordan, retiradas de [28], e que deve serem lidas da seguinte forma :

\begin{tabular}{c|c|cc}
\hline \hline & $\mathrm{N}$ & $\left(m_{1} m_{2}\right)$ & $\ldots$ \\
\hline$[v] \tau, m$ & $N_{i}$ & $c_{i}$ & $\ldots$ \\
$\vdots$ & $\vdots$ & $\vdots$ & \\
\hline \hline
\end{tabular}

onde aqui $\mathrm{N}$ é o coeficiente de normalização de modo então que o coeficiente de ClebschGordan seje dado por $\sqrt{c_{i} / \Lambda_{i}}$. Assim sendo : 


\begin{tabular}{c|c|cc}
\hline \hline & $\mathrm{N}$ & $(11)$ & $(22)$ \\
\hline$[3]$ & 2 & 1 & 1 \\
{$[21] 1$} & 2 & 1 & $* 1$ \\
\hline & & $(12)$ & $(21)$ \\
\hline$[21] 2$ & 2 & $* 1$ & $* 1$ \\
{$\left[1^{3}\right]$} & 2 & 1 & $* 1$ \\
\hline \hline
\end{tabular}

Tabela 4.5: Coeficientes de Clebsch-Gordan : $[21] \times[21]=[3]+[21]+\left[1^{3}\right]$.

\begin{tabular}{c|c|ccccc}
\hline \hline & $\mathrm{N}$ & $(11)$ & $(12)$ & $(21)$ & $(22)$ & $(33)$ \\
\hline$[4]$ & 3 & 1 & & & 1 & 1 \\
{$[31] 1$} & 6 & 4 & & & $* 1$ & $* 1$ \\
{$[31] 2$} & 6 & & $* 1$ & $* 1$ & 2 & $* 2$ \\
{$\left[2^{2}\right] 1$} & 6 & & 2 & 2 & 1 & $* 1$ \\
{$\left[21^{2}\right] 1$} & 2 & & 1 & $* 1$ & & \\
\hline & & $(13)$ & $(31)$ & $(23)$ & $(32)$ & \\
\hline$[31] 3$ & 6 & $* 1$ & $* 1$ & $* 2$ & $* 2$ & \\
{$\left[2^{2}\right] 2$} & 6 & 2 & 2 & $* 1$ & $* 1$ & \\
{$\left[21^{2}\right] 2$} & 2 & 1 & $* 1$ & & & \\
{$\left[21^{2}\right] 3$} & 2 & & & 1 & $* 1$ & \\
\hline \hline
\end{tabular}

Tabela 4.6: Coeficientes de Clebsch-Gordan : $[31] \times[31]=[4]+[31]+\left[2^{2}\right]+\left[21^{2}\right]$.

\begin{tabular}{c|c|ccc}
\hline \hline & $\mathrm{N}$ & $(11)$ & $(21)$ & $(32)$ \\
\hline$[31] 1$ & 2 & & 1 & 1 \\
{$[31] 2$} & 4 & 2 & 1 & $* 1$ \\
{$\left[21^{2}\right] 1$} & 4 & 2 & $* 1$ & 1 \\
\hline & & $(12)$ & $(22)$ & $(31)$ \\
\hline$[31] 3$ & 4 & 2 & $* 1$ & $* 1$ \\
{$\left[21^{2}\right] 2$} & 4 & 2 & 1 & 1 \\
{$\left[21^{2}\right] 3$} & 2 & & $* 1$ & 1 \\
\hline \hline
\end{tabular}

Tabela 4.7: Coeficientes de Clebsch-Gordan : [31] $\times\left[2^{2}\right]=[31]+\left[21^{2}\right]$. 


\begin{tabular}{c|c|ccccc|ccccc}
\hline \hline & $\mathrm{N}$ & $(11)$ & $(12)$ & $(13)$ & $(21)$ & $(22)$ & $(23)$ & $(31)$ & $(32)$ & $(33)$ & $(44)$ \\
\hline$[5]$ & 4 & 1 & & & & 1 & & & & 1 & 1 \\
{$[41] 1$} & 12 & 9 & & & & $* 1$ & & & & $* 1$ & $* 1$ \\
{$[41] 2$} & 36 & & $* 3$ & & $* 3$ & 20 & & & & $* 5$ & $* 5$ \\
{$[41] 3$} & 36 & & & $* 3$ & & & $* 5$ & $* 3$ & $* 5$ & 10 & $* 10$ \\
\hline$[32] 1$ & 36 & & 15 & & 15 & 4 & & & & $* 1$ & $* 1$ \\
{$[32] 2$} & 36 & & & 15 & & & $* 1$ & 15 & $* 1$ & 2 & $* 2$ \\
{$[32] 4$} & 6 & & & & & & 2 & & 2 & 1 & $* 1$ \\
\hline$\left[31^{2}\right] 1$ & 2 & & 1 & & $* 1$ & & & & & & \\
{$\left[31^{2}\right] 2$} & 2 & & & 1 & & & & $* 1$ & & & \\
{$\left[31^{2}\right] 4$} & 2 & & & & & & 1 & & $* 1$ & & \\
\hline$[41] 4$ & 36 & $* 3$ & $* 5$ & $* 10$ & $* 3$ & $* 5$ & $* 10$ & & & & \\
{$[32] 3$} & 36 & 15 & $* 1$ & $* 2$ & 15 & $* 1$ & $* 2$ & & & & \\
{$[32] 5$} & 6 & & 2 & $* 1$ & & 2 & $* 1$ & & & & \\
\hline$\left[31^{2}\right] 3$ & 2 & 1 & & & $* 1$ & & & & & & \\
{$\left[31^{2}\right] 5$} & 2 & & 1 & & & $* 1$ & & & & & \\
{$\left[31^{2}\right] 6$} & 2 & & & 1 & & & $* 1$ & & & & \\
\hline \hline
\end{tabular}

Tabela 4.8: Coeficientes de Clebsch-Gordan : $[41] \times[41]=[5]+[41]+[32]+\left[31^{2}\right]$. 
onde * denota que o coeficiente é negativo. Por exemplo, da tabela (4.5) tiramos os seguimtes Clebsch-Gordan :

$$
\begin{gathered}
C_{[21] 2,[21] 2}^{[21] 1}=-\sqrt{\frac{1}{2}} \\
C_{[21] 1,[21] 1}^{[21] 1}=\sqrt{\frac{1}{2}}
\end{gathered}
$$

da tabela (4.7) :

$$
\begin{aligned}
& C_{[32] 2,[22] 1}^{\left[21^{2}\right] 1}=-\sqrt{\frac{1}{4}} \\
& C_{[32] 2,[22] 1}^{[31] 2}=\sqrt{\frac{1}{4}}
\end{aligned}
$$

e da tabela (4.8) :

$$
\begin{aligned}
& C_{[41] 1,[41] 2}^{[41] 2}=-\sqrt{\frac{3}{36}} \\
& C_{[41] 3,[41] 4}^{[32] 3}=-\sqrt{\frac{2}{36}} .
\end{aligned}
$$

Os casos analisados anteriormente são casos em que $\tau=1$, e que portanto omitimos este na notação.

Cada tabela pode então ser lida horizontalmente, afim de se determinar as bases $\Psi_{m}^{[\nu]}$. Por exemplo para o produto $[21] \times[21]=[3]+\left[21+\left[1^{3}\right]\right.$ teremos as bases :

$$
\Psi^{[3]}=\frac{1}{\sqrt{2}}\left(\psi_{1}^{[21]} \varphi_{1}^{[21]}+\psi_{2}^{[21]} \varphi_{2}^{[21]}\right)
$$

para a representação simétrica,

$$
\begin{aligned}
\Psi_{1}^{[21]} & =\frac{1}{\sqrt{2}}\left(\psi_{1}^{[21]} \varphi_{1}^{[21]}-\psi_{2}^{[21]} \varphi_{2}^{[21]}\right) \\
\Psi_{2}^{[21]} & =-\frac{1}{\sqrt{2}}\left(\psi_{1}^{[21]} \varphi_{2}^{[21]}+\psi_{2}^{[21]} \varphi_{1}^{[21]}\right)
\end{aligned}
$$

para a representação mista bidimensional, e

$$
\Psi^{\left[1^{3}\right]}=\frac{1}{\sqrt{2}}\left(\psi_{1}^{[21]} \varphi_{2}^{[21]}-\psi_{2}^{[21]} \varphi_{1}^{[21]}\right)
$$

para a representação anti-simétrica. Da tabela (4.8) temos por exemplo :

$$
\begin{aligned}
\Psi_{2}^{[32]}=\frac{1}{\sqrt{36}}\left(\sqrt{15} \psi_{1}^{[41]} \varphi_{3}^{[41]}-\psi_{2}^{[41]} \varphi_{3}^{[41]}+\sqrt{15} \psi_{3}^{[41]} \psi_{1}^{[41]}-\psi_{3}^{[41:} \psi_{2}^{[41]}\right. & +\sqrt{2} \psi_{3}^{[41]} \psi_{3}^{[41]} \\
& \left.-\sqrt{2} \psi_{4}^{[41]} \varphi_{4}^{[41]}\right)
\end{aligned}
$$

de modo então a termos as bases irredutíveis para as representações contidas no produto de Kronecker. 


\section{Capítulo 5}

\section{Simetrização das Funções de Canal}

Iremos neste capítulo começar a analisar a simetria da nossa função de onda, mais especificamente das funções de canal $\Phi_{n}(R ; \Omega)$. A investigação começa ao analisarmos as simetrias das funções de spin nas funções de canal, dadas por :

$$
\Phi_{n}(R ; \Omega)=\sum_{\tau^{\prime}} \Delta_{n}^{\tau^{\prime}}(R) \varphi_{\tau^{\prime}}(\Omega)
$$

onde os seus coeficientes são simétricos, com relação à troca de partículas, e a sua base $\left\{\varphi_{\tau}\right\}$ é formada pelo produto $\chi^{(\text {Spin) }} \otimes \varphi^{(\text {Angular })}$. Deste modo, conhecendo a simetria das funções de spin poderemos determinar que simetria as funções angulares devem portar para que o produto de Kronecker de ambas possa nos dar uma função anti-simétrica. Mostraremos aqui somente quais devem ser as representações que a parte de spin e angular devem porta: para obtermos uma representação anti-simétrica. e deixaremos para o próximo capítulo a determinação da base para esta.

\subsection{Produto de Kronecker : Spin $\otimes$ Angular}

O grupo de Permutação $\mathbf{S}_{\mathbf{3}}$, tem uma aplicação natural tanto sobre as posições comó nos spins dos elétrons, para o caso do átomo de lítio. Sabemos que o grupo $\mathbf{S}_{3}$ tem três representações irredutíveis, cada uma associada a um Young Tableau :

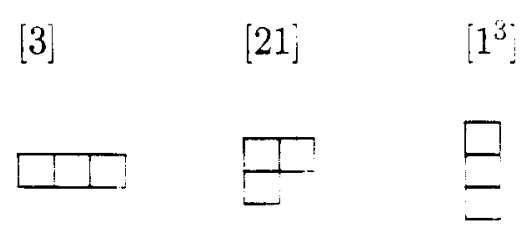


onde [3] é a representação simétrica, unidimensional, [21] a mista, bidimensional, e [1 $\left.{ }^{3}\right]$ a representação anti-simétrica, unidimensional, conforme discutido no capítulo 4 .

$\mathrm{O}$ átomo de lítio tem estados de spin total $1 / 2$ e $3 / 2$. O ground state e suas excitações tem spin total $1 / 2$ e será onde concentraremos nossa atenção. Caso este, que implica na simetria mista das funções de spin, e que portanto devem ser representada pelo seguinte Young Tableau :

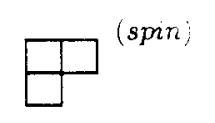

associado à representação irredutível [21]. Da decomposição do produto de Kronecker nas suas componentes irredutíveis, séries de Clebsch-Gordan, temos conforme mostrado na seção 4.7.1 :

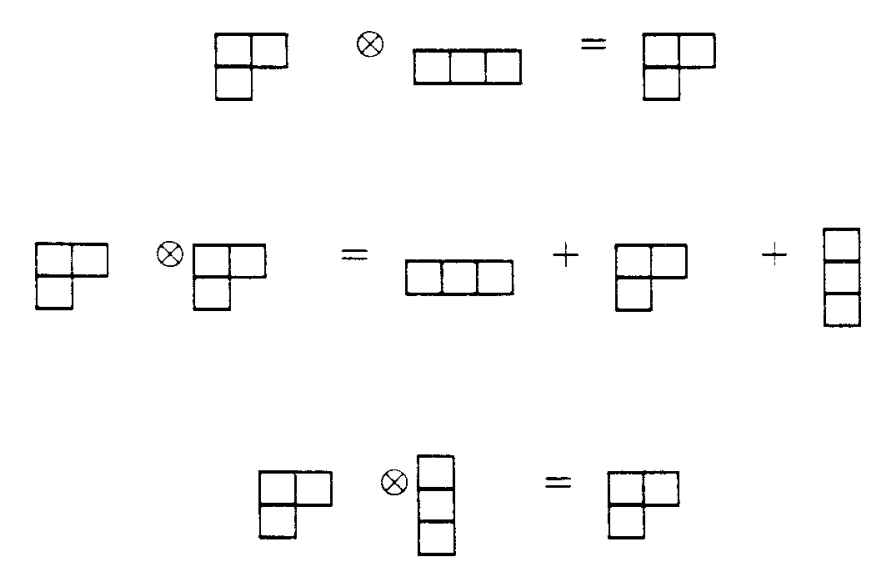

de modo a podermos deduzir que sendo uma das representações a irrep mista, o produto de Kronecker irá conter uma representação irredutivel anti-simétrica, $\left[1^{3}\right]$, somente se a outra representação também for uma representação mista, equação (5.3). O que implica então na imposição da simetria mista também para a função angular. de forma que o produto

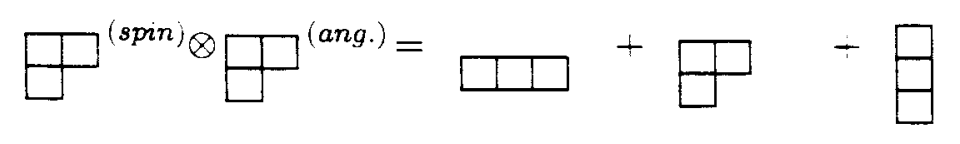

venha a conter uma representeção anti-simétrica, a qual nos torna possível obter o princípïo de exclusão de Pauli ao se determinar a base irredutivel para esta representação.

Podemos determinar a base que carrega a simetria desejada através dos Operadores de Projeção. seção 4.6. A procura desta base será feita tanto para a parte angular como para a parte spinorial, pois o resultado da composição de ambas é que deverá conter uma representação totalmente Anti-Simétrica, satisfazendo deste modo o Princípio de Exclusão de Pauli. 
No caso das funções de spin, podemos determinar a base que porta a representação irredutível [21] a partir dos Coeficientes de Clebsch-Gordan para a soma de três spins 1/2, $S U(2) \otimes S U(2) \otimes S U(2),[3]$ e [27], sendo este o caminho adotados para os nossos cálculos aqui.

Para as funções angulares o problema de determinar as bases irredutíveis da simetria mista [21] é atacado através do uso dos operadores de projeção tendo definido a representação matricial (unitária) a ser utilizada e estabelecendo a atuação dos operadores do grupo conforme discutido na seção $\mathbf{4 . 4}$.

\subsection{Simetria Mista da Função de Spin}

Ao realizarmos a adição de três spins $1 / 2$, utilizando os coeficientes de Clebsch-Gordan e sob a seguinte notação para as funções de spin : $\left|s_{12} S M_{S}\right\rangle, s_{12}$ para o spin intermediario (0 ou 1 ) e $S$ para o spin total ( $1 / 2$ ou $3 / 2$ ); obtemos o seguinte resultado

- $s_{12}=0 \oplus 1 / 2$

$$
\mathbf{S}=1 / 2\left\{\begin{array}{l}
|0,1 / 2,1 / 2\rangle=\frac{1}{\sqrt{2}}(|+-+\rangle-|-++\rangle) \\
|0,1 / 2,-1 / 2\rangle=\frac{1}{\sqrt{2}}(|+--\rangle-|-+-\rangle)
\end{array}\right.
$$

- $s_{12}=1 \oplus 1 / 2$

$$
\mathbf{S}=\mathbf{3} / \mathbf{2}\left\{\begin{array}{c}
|1,3 / 2,3 / 2\rangle=|+++\rangle \\
|1,3 / 2,1 / 2\rangle=\frac{1}{\sqrt{3}}(|+-+\rangle+|-++\rangle+|++-\rangle) \\
|1,3 / 2,-1 / 2\rangle=\frac{1}{\sqrt{3}}(|--+\rangle+|---\rangle+|-+-\rangle)
\end{array}\right.
$$




\section{- Representação Mista}

Se aplicarmos o elementos do grupo de permutação $\mathbf{S}_{\mathbf{3}}$, veremos que as funções de spin portam duas representações mistas bidimensionais equivalentes. As duas bases para as representações mista são :

$$
(|1,1 / 2,1 / 2\rangle,|0,1 / 2,1 / 2\rangle) \text { e }(|1,1 / 2,-1 / 2\rangle,|0,1 / 2,-1 / 2\rangle)
$$

Assim sendo cada um dos pares acima porta a seguinte representação matricial do $\mathbf{S}_{\mathbf{3}}$. [29] :

$$
D(12)=\left(\begin{array}{cc}
1 & 0 \\
0 & -1
\end{array}\right), \quad D(23)=\left(\begin{array}{cc}
-1 / 2 & \sqrt{3} / 2 \\
\sqrt{3} / 2 & 1 / 2
\end{array}\right),
$$

que já caracterizam a representação matricial pelo fato de que (12) e (23) serem os geradores do $\mathbf{S}_{\mathbf{3}}$.

Devido ao fato de que o hamiltoniano (2.1) independe dos spin eletrônicos contruiremos a função spinorial, para o estado fudamental do lítio, da seguinte forma

$$
\begin{aligned}
& \chi_{1}=\frac{1}{\sqrt{2}}(|1,1 / 2,1 / 2\rangle+|1,1 / 2,-1 / 2\rangle) \\
& \chi_{2}=\frac{-1}{\sqrt{2}}(|0,1 / 2,1 / 2\rangle+|0,1 / 2,-1 / 2\rangle),
\end{aligned}
$$

de modo que as componentes $S_{z}$ tenham o mesmo peso. Assim sendo teremos outra representação matricial associada à esta base, a qual é dada por :

$$
D(12)=\left(\begin{array}{cc}
1 & 0 \\
0 & -1
\end{array}\right), \quad D(23)=\left(\begin{array}{cc}
-1 / 2 & -\sqrt{3} / 2 \\
-\sqrt{3} / 2 & 1 / 2
\end{array}\right),
$$

e que portanto dever ser também adotada para a representação da parte angular.

\subsection{Simetria Mista da Função Angular}

Determinamos a atuação do grupo de permutação no espaço das funções na seção 4.4. Adotamos neste trabalho o produto $(D-E)$ para os elementos do grupo e que implica na seguinte ação dos operadores :

$$
R \phi(\mathbf{x})=\phi(R \mathbf{x})
$$




\subsection{SIMETRIA MISTA DA FUNÇÃO ANGULAR}

Assim fizemos por ser a multiplição (D-E) a mais usual.

Para as funções angulares, determinaremos as bases irredutíveis via os Operadores de Projeção, discutidos anteriormente. A representação matricial a ser utilizada deve ser igual à representação determinada para a parte spinorial, equação (5.10), mantendo a concordância, o que é de fundamental importância.

\section{- A Representação Mista :}

$\mathrm{Na}$ construção das bases para a representação mista, temos a seguinte representação matricial :

$$
\begin{array}{ll}
D(e)=\left(\begin{array}{ll}
1 & 0 \\
0 & 1
\end{array}\right) & D(12)=\left(\begin{array}{cc}
1 & 0 \\
0 & -1
\end{array}\right) \\
D(13)=\left(\begin{array}{cc}
-1 / 2 & \sqrt{3} / 2 \\
\sqrt{3} / 2 & 1 / 2
\end{array}\right) & D(23)=\left(\begin{array}{cc}
-1 / 2 & -\sqrt{3} / 2 \\
-\sqrt{3} / 2 & 1 / 2
\end{array}\right) \\
D(123)=\left(\begin{array}{cc}
-1 / 2 & -\sqrt{3} / 2 \\
\sqrt{3} / 2 & -1 / 2
\end{array}\right) & D(132)=\left(\begin{array}{cc}
-1 / 2 & \sqrt{3} / 2 \\
-\sqrt{3} / 2 & -1 / 2
\end{array}\right)
\end{array}
$$

e assim sendo obtemos para esta os seguintes operadores de projeção :

$$
\begin{aligned}
\rho_{11}^{(m)} & =\frac{1}{3}\left[e+(12)-\left(\frac{(13)+(23)+(123)+(132)}{2}\right)\right], \\
\rho_{21}^{(m)} & =\frac{\sqrt{3}}{6}[(13)-(23)+(123)-(132)], \\
\rho_{12}^{(m)} & =\frac{\sqrt{3}}{6}[(13)-(23)-(123)+(132)], \\
\rho_{22}^{(m)} & =\frac{1}{3}\left[e-(12)+\left(\frac{(13)+(23)-(123)-(132)}{2}\right)\right],
\end{aligned}
$$

e portanto :

$$
\begin{aligned}
\varphi_{11}^{(m)} & =\rho_{11}^{(m)} \phi\left(r_{1}, r_{2}, r_{3}\right)= \\
& =\frac{1}{6}\left\{2 \phi\left(r_{1}, r_{2}, r_{3}\right)+2 \phi\left(r_{2}, r_{1}, r_{3}\right)-\phi\left(r_{3}, r_{2}, r_{1}\right)-\phi\left(r_{1}, r_{3}, r_{2}\right)-\right. \\
& \left.-\phi\left(r_{2}, r_{3}, r_{1}\right)+-\phi\left(r_{3}, r_{1}, r_{2}\right)\right\}
\end{aligned}
$$




$$
\begin{aligned}
\varphi_{21}^{(m)} & =\rho_{21}^{(m)} \phi\left(r_{1}, r_{2}, r_{3}\right)= \\
& =\frac{\sqrt{3}}{6}\left\{\phi\left(r_{3}, r_{2}, r_{1}\right)-\phi\left(r_{1}, r_{3}, r_{2}\right)+\phi\left(r_{2}, r_{3}, r_{1}\right)-\phi\left(r_{3}, r_{1}, r_{2}\right)\right\}, \\
\varphi_{12}^{(m)} & =\rho_{12}^{(m)} \phi\left(r_{1}, r_{2}, r_{3}\right)= \\
& =\frac{\sqrt{3}}{6}\left\{\phi\left(r_{3}, r_{2}, r_{1}\right)-\phi\left(r_{1}, r_{3}, r_{2}\right)-\phi\left(r_{2}, r_{3}, r_{1}\right)+\phi\left(r_{3}, r_{1}, r_{2}\right)\right\}, \\
\varphi_{22}^{(m)} & =\rho_{22}^{(m)} \phi\left(r_{1}, r_{2}, r_{3}\right)= \\
& =\frac{1}{6}\left\{2 \phi\left(r_{1}, r_{2}, r_{3}\right)-2 \phi\left(r_{2}, r_{1}, r_{3}\right)+\phi\left(r_{3}, r_{2}, r_{1}\right)+\phi\left(r_{1}, r_{3}, r_{2}\right)-\right. \\
& \left.-\phi\left(r_{2}, r_{3}, r_{1}\right)-\phi\left(r_{3}, r_{1}, r_{2}\right)\right\},
\end{aligned}
$$

onde cada par $\left\{\varphi_{11}^{(m)}, \varphi_{21}^{(m)}\right\}$ e $\left\{\varphi_{12}^{(m)}, \varphi_{22}^{(m)}\right\}$, correspondem aos pares de partners $\left\{\rho_{11}^{(m)}, \rho_{21}^{(m)}\right\}$ $\mathrm{e}\left\{\rho_{12}^{(m)}, \rho_{22}^{(m)}\right\}$ respectivamente, carrega a mesma irrep mista do $\mathbf{S}_{\mathbf{3}}$. Para o nosso caso, a função $\phi$ é justamente o hiperesférico harmônico do $S O(9)$ discutido na seção 2.1, e que em coordenadas esféricas são dados por :

$$
\begin{aligned}
\phi_{\mu \nu l_{1} l_{2} l_{3} l_{12}}^{L M}\left(r_{1}, r_{2}, r_{3}\right)= & N_{\mu}^{l_{1} l_{2}} N_{\mu \nu}^{l_{1} l_{2} l_{3}} r_{1}^{l_{1}} r_{2}^{l_{2}} r_{3}^{l_{3}}\left(r_{1}^{2}+r_{2}^{2}\right)^{\mu} R^{2 \nu} P_{\mu}^{\left(l_{1}+1 / 2, l_{2}+1 / 2\right)}\left(\frac{r_{2}^{2}-r_{1}^{2}}{r_{1}^{2}+r_{2}^{2}}\right) \\
& P_{\nu}^{\left(2 \mu+l_{1}+l_{2}+2, l_{3}+1 / 2\right)}\left(\frac{r_{3}^{2}-r_{2}^{2}-r_{1}^{2}}{R^{2}}\right) \mathcal{Y}_{l_{1} l_{2} l_{3} l_{12}}^{L M}\left(\Lambda_{1}, \Lambda_{2}, \Lambda_{3}\right)
\end{aligned}
$$

Denotaremos para a atuação do Operador de Projeção num elemento da base em questão como :

$$
\begin{aligned}
\varphi_{\tau}^{(11)} & \equiv \rho_{11}^{(m)} \phi_{\mu \nu l_{1} l_{2} l_{3} l_{12}}^{L M} \\
\varphi_{\tau}^{(21)} & \equiv \rho_{21}^{(m)} \phi_{\mu \nu l_{1} l_{2} l_{3} l_{12}}^{L M} \\
\varphi_{\tau}^{(12)} & \equiv \rho_{12}^{(m)} \phi_{\mu \nu l_{1} l_{2} l_{3} l_{12}}^{L M} \\
\varphi_{\tau}^{(22)} & \equiv \rho_{22}^{(m)} \phi_{\mu \nu l_{1} l_{2} l_{3} l_{12}}^{L M}
\end{aligned}
$$

sendo $\tau$ o índice que rotula as funções projetadas. Para o conjunto de números quânticos $\left\{\mu \nu l_{1} l_{2} l_{3}\right\}$ está relacionado um valor de $\lambda_{9}=2 \mu+2 \nu+l_{1}+l_{2}+l_{3}$. Para cada valor de $\lambda_{9}$ está associado um espaço vetorial que porta uma representação irredutível do $S O(9)$, e que contém as combinações de $\left\{\mu \nu l_{1} l_{2} l_{3} l_{12}\right\}$ que dão o determinado $\lambda_{9}$. No entanto a atuação de uma permutação nas funções $\phi_{\mu \nu l_{1} l_{2} l_{3} l_{12}}^{L M}$ nos dá uma combinação linear destas, as quais pertencem à mesma representação do $S O(9)$, isto é. uma combinação das funções com mesmo 
valor de $\lambda_{9}$, de modo a que estas ainda venham a ser auto-funções do operador de Casimir do grupo $S O(9)$, conforme pode ser visto por

$$
R \hat{\mathbf{U}}(0) \phi_{\mu \nu l_{1} l_{2} l_{3} l_{12}}^{L M}=\hat{\mathbf{U}}(0) R \phi_{\mu \nu l_{1} l_{2} l_{3} l_{12}}^{L M}=-\left(\lambda_{9}+7 / 2\right)^{2} R \phi_{\mu \nu l_{1} l_{2} l_{3} l_{12}}^{L M}
$$

devido à invariança da energia cinética sob a atuação do grupo de permutação $\mathbf{S}_{3}$. Denotaremos portanto a ação da permutação por :

$$
R \phi_{\mu \nu l_{1} l_{2} l_{3} l_{12}}^{L M}=\sum_{\mu^{\prime} \nu^{\prime} l_{1}^{\prime} l_{2}^{\prime} l_{3}^{\prime} l_{12}^{\prime}} A_{\mu^{\prime} \nu^{\prime} l_{1}^{\prime} l_{2}^{\prime} l_{3}^{\prime} l_{12}^{\prime} \mu \nu l_{1} l_{2} l_{3} l_{12}}^{R} \phi_{\mu^{\prime} \nu^{\prime} l_{1}^{\prime} l_{2}^{\prime} l_{3}^{\prime} l_{12}^{\prime}}^{L M}
$$

O que nos interessa determinar são os coeficientes $A_{\eta^{\prime}, \eta}^{R}, \eta \equiv\left\{\mu \nu l_{1} l_{2} l_{3} l_{12}\right\}$. A atuação de uma permutação em $\mathcal{Y}\left(\Lambda_{1}, \Lambda_{2}, \Lambda_{3}\right)$ nos dá expressões em termos dos $6-j$ símbolos, devido à dependência dos $\mathcal{Y}$ nos coeficientes de Clebsch-Gordan. Já para a atuação nas funções provindas das partes em $\alpha$ e $\beta$, temos um caso um pouco mais complicado, de modo que iremos aqui analisar esta atuação resolvendo as integrais que determinam o valor das componentes da combinação linear resultante da referida atuação. Assim fazendo obtemos para o nosso caso :

$$
\begin{aligned}
& A_{\eta^{\prime}, \eta}^{e}=\delta_{\mu \mu^{\prime}} \delta_{\nu \nu^{\prime}} \delta_{l_{1} l_{1}^{\prime}} \delta_{l_{2} l_{2}^{\prime}} \delta_{l_{3} l_{3}^{\prime}} \delta_{l_{12} l_{12}^{\prime}} \\
& A_{\eta^{\prime}, \eta}^{(12)}=(-1)^{\mu+l_{1}+l_{2}+l_{12}} \delta_{\mu \mu^{\prime}} \delta_{\nu \nu^{\prime}} \delta_{l_{1} l_{2}^{\prime}} \delta_{l_{2} l_{1}^{\prime}} \delta_{l_{3} l_{3}^{\prime}} \delta_{l_{12} l_{12}^{\prime}} \\
& A_{\eta^{\prime}, \eta}^{(13)}=\delta_{l_{1} l_{3}^{\prime}} \delta_{l_{2} l_{2}^{\prime}} \delta_{l_{3} l_{1}^{\prime}} \sqrt{\left(2 l_{12}+1\right)\left(2 l_{12}^{\prime}+1\right)}\left\{\begin{array}{l}
l_{12} l_{3} L \\
l_{12}^{\prime} l_{1} l_{2}
\end{array}\right\} \\
& \int_{0}^{\pi / 2} \int_{0}^{\pi / 2} g_{\mu^{\prime} l_{1}^{\prime} l_{2}^{\prime}}^{*}(\alpha) f_{\mu^{\prime} \nu^{\prime} l_{1}^{\prime} l_{2}^{\prime} l_{3}^{\prime}}(\beta)(\mathbf{1 3}) g_{\mu l_{1} l_{2}}(\alpha) f_{\mu \nu l_{1} l_{2} l_{3}}(\beta) \\
& \sin ^{2} \alpha \cos ^{2} \alpha \sin ^{5} \beta \cos ^{2} \beta d \alpha d \beta,
\end{aligned}
$$

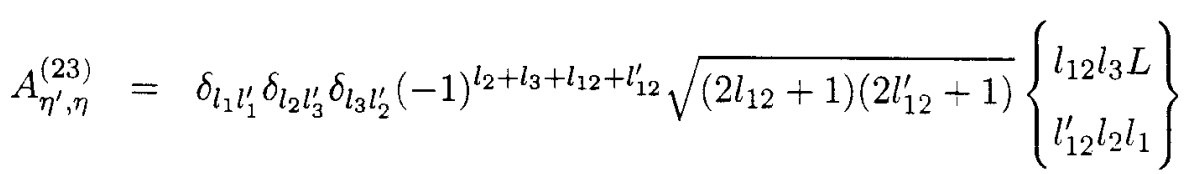

$$
\begin{aligned}
& \int_{0}^{\pi / 2} \int_{0}^{\pi / 2} g_{\mu^{\prime} l_{1}^{\prime} l_{2}^{\prime}}^{*}(\alpha) f_{\mu^{\prime} \nu^{\prime} l_{1}^{\prime} l_{2}^{\prime} l_{3}^{\prime}}^{*}(\beta)(\mathbf{2 3}) g_{\mu l_{1} l_{2}}(\alpha) f_{\mu \nu l_{1} l_{2} l_{3}}(\beta) \\
& \sin ^{2} \alpha \cos ^{2} \alpha \sin ^{5} \beta \cos ^{2} \beta d \alpha d \beta,
\end{aligned}
$$

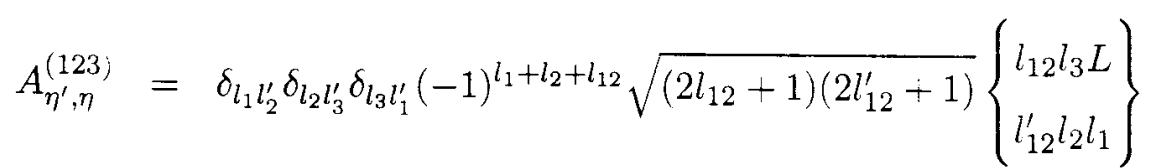

$$
\begin{aligned}
& \int_{0}^{\pi / 2} \int_{0}^{\pi / 2} g_{\mu^{\prime} l_{1}^{\prime} l_{2}^{\prime}}^{*}(\alpha) f_{\mu^{\prime} \nu^{\prime} l_{1}^{\prime} l_{2}^{\prime} l_{3}^{\prime}}^{*}(\beta)(\mathbf{1 2 3}) g_{\mu l_{1} l_{2}}(\alpha) f_{\mu \nu l_{1} l_{2} l_{3}}(\beta) \\
& \sin ^{2} \alpha \cos ^{2} \alpha \sin ^{5} \beta \cos ^{2} \beta d \alpha d \beta,
\end{aligned}
$$




$$
\begin{aligned}
& A_{\eta^{\prime}, \eta}^{(132)}=\delta_{l_{1} l_{3}^{\prime}} \delta_{l_{2} l_{3}^{\prime}} \delta_{l_{3} l_{2}^{\prime}}(-1)^{l_{2}+l_{3}+l_{12}^{\prime}} \sqrt{\left(2 l_{12}+1\right)\left(2 l_{12}^{\prime}+1\right)}\left\{\begin{array}{l}
l_{12} l_{3} L \\
l_{12}^{\prime} l_{1} l_{2}
\end{array}\right\} \\
& \int_{0}^{\pi / 2} \int_{0}^{\pi / 2} g_{\mu^{\prime} l_{1}^{\prime} l_{2}^{\prime}}^{*}(\alpha) f_{\mu^{\prime} \nu^{\prime} l_{1}^{\prime} l_{2}^{\prime} l_{3}^{\prime}}^{*}(\beta)(\mathbf{1 3 2}) g_{\mu l_{1} l_{2}}(\alpha) f_{\mu \nu l_{1} l_{2} l_{3}}(\beta) \\
& \sin ^{2} \alpha \cos ^{2} \alpha \sin ^{5}, 3 \cos ^{2}, 3 d \alpha d \beta,
\end{aligned}
$$

onde usamos novamente as relações de [3]. As integrais em $\alpha$ e $\beta$ são calculadas num programa em Maple, no qual primeiramente determinamos a atuação da permutação na função em coordenadas esféricas, e posteriormente voltamos para as coordenas hiperesféricas para assinı resolver o nosso problema. Assim sendo poderemos calcular as funções $\varphi^{(i j)}$ de acordo com o projetor que iremos utilizar, tendo :

$$
\varphi_{\tau}^{(i j)}=\frac{1}{3} \sum_{G, \mu^{\prime} \nu^{\prime} l_{1}^{\prime} l_{2}^{\prime} l_{3}^{\prime} l_{12}^{\prime}} D_{i j}^{(m)}(G) A_{\mu^{\prime} \nu^{\prime} l_{1}^{\prime} l_{2}^{\prime} l_{3}^{\prime} l_{12}^{\prime}, \tau}^{G} \phi_{\mu^{\prime} \nu^{\prime} l_{1}^{\prime} l_{2}^{\prime} l_{3}^{\prime} l_{12}^{\prime}}^{L}
$$

para a sua forma geral.

Conforme vimos, temos duas bases distintas que portam a mesma representação mista $\left\{\varphi^{(11)}, \varphi^{(21)}\right\}$ e $\left\{\varphi^{(12)}, \varphi^{(22)}\right\}$. Mas precisamos de apenas um par, já que carregam a mesma representação. Assim escolhendo o par $\left\{\varphi^{(11)}, \varphi^{(21)}\right\}$, para a nossa função angular, e denotaremos daqui em diante este por

$$
\left\{\varphi_{\tau}^{(1)}, \varphi_{\tau}^{(2)}\right\}
$$

Um motivo pelo qual não escolhemos para a parte angular uma base que fosse uma combinação dos pares é que ambos pares de projetores $\left\{\rho_{11}^{(m)}, \rho_{21}^{(m)}\right\}$ e $\left\{\rho_{12}^{(m)}, \rho_{22}^{(m)}\right\}$ geram a mesma base, isto é, as funções de bases são para este caso iguais, diferentemente do que acontece com os spins. No entanto o operador de projeção aplicado a um conjunto de funções ortogonais destroi a ortogonalidade das funções originais, o qual é o caso da aplicação dos operadores de projeção do grupo $\mathbf{S}_{3}$ nos hiperesféricos do $S O(9)$. Portanto, ao aplicarmos o operador de projeção $\rho_{11}^{(m)}$ nos hiperesféricos harmônicos pertencentes a um mesmo valor de $\lambda_{9}$ deveremos ortonormalizar estas novas funções de modo a obtermos uma base ortogonal tanto para as representações do grupo de permutação como para o grupo de rotações. 


\section{Capítulo 6}

\section{Anti-Simetria da Função de Onda}

\section{Total}

Nesta seção iremos construir a nossa função de onda total anti-simétrica a partir do conhecimento da simetria das funções angular e de spin.

Portanto tendo a série de Clebsch-Gordan para o produto de Kronecker do nosso problema

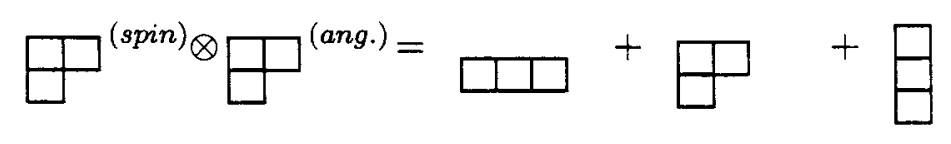

queremos determinar a base para a representação anti-simétrica, contido no produto, de forma que esta venha a constituir uma base para a nossa função de canal, e nos dando por final a anti-simetria para a função de onda total.

Assim sendo temos em questão o problema de Clebsch-Gordon, o qual foi analisado no capítulo 4, seção 4.7.2, onde foi analisado o nosso caso como um exemplo. Portanto tendo a tabela (4.5), para os coeficientes de Clebsh-Gordan, a equação (4.108) nos dará como base para a representação anti-simétrica $\left[1^{3}\right]$ a seguinte função :

$$
\varphi^{\left[1^{3}\right]}=\frac{1}{\sqrt{2}}\left(\chi_{1}^{[21]} \varphi_{2}^{[21]}-\chi_{2}^{[21]} \varphi_{1}^{[21]}\right) .
$$

Para o nosso caso, a parte angular como base as seguintes funções

$$
\begin{aligned}
& \varphi_{\tau}^{(1)}=\rho_{11}^{(m)} \phi_{\mu \nu l_{1} l_{2} l_{3} l_{12}}^{L M} \\
& \varphi_{\tau}^{(2)}=\rho_{21}^{(m)} \phi_{\mu \nu l_{1} l_{2} l_{3} l_{12}}^{L M}
\end{aligned}
$$


e a dos spins :

$$
\begin{aligned}
& \chi_{1}=\frac{1}{\sqrt{2}}(|1,1 / 2,1 / 2\rangle+|1,1 / 2,-1 / 2\rangle) \\
& \chi_{2}=\frac{-1}{\sqrt{2}}(|0,1 / 2,1 / 2\rangle+|0,1 / 2,-1 / 2\rangle),
\end{aligned}
$$

conforme visto anteriormente. Portanto teremos como base para a representação anti-simétria a seguinte função :

$$
\varphi_{\tau}=\frac{1}{\sqrt{2}}\left(\varphi_{\tau}^{(1)} \chi_{2}-\varphi_{\tau}^{(2)} \chi_{1}\right)
$$

de modo então que a nossa função de canal :

$$
\Phi_{n}(R ; \Omega)=\sum_{\tau^{\prime}} \Delta_{n}^{\tau^{\prime}}(R) \varphi_{\tau^{\prime}}(\Omega)
$$

expandida na base $\left\{\varphi_{\tau}\right\}$, venha a ser anti-simétrica, de modo que a nossa função de onda total também será anti-simétrica:

$$
\Psi(R ; \Omega)=\sum_{\nu} F_{\nu}(R) \Phi_{\nu}(R ; \Omega)
$$

\section{- A anti-simetria :}

Sendo $R^{(e s p)}$ e $R^{(s p i n)}$ os operadores do $\mathbf{S}_{\mathbf{3}}$ que atuam respectivamente na parte espacial e na de spin, temos:

$$
\begin{aligned}
R^{(e s p)} \varphi_{\tau}^{\mu} & =\sum_{\nu=1}^{2} \varphi_{\tau}^{\nu} D_{\nu \mu}^{(m)}(R) \\
R^{(s p i n)} \chi_{\mu} & =\sum_{\nu=1}^{2} \chi_{\nu} D_{\nu \mu}^{(m)}(R)
\end{aligned}
$$

então :

$$
\begin{aligned}
R^{(e s p)} R^{(s p i n)} \varphi_{\tau} & = \\
& =\frac{1}{\sqrt{2}} \sum_{\nu, \lambda=1}^{2} \varphi_{\tau}^{\nu} \chi_{\lambda}\left[D_{\nu 1}^{(m)}(R) D_{\lambda 2}^{(m)}(R)-D_{\nu 2}^{(m)}(R) D_{\lambda 1}^{(m)}(R)\right]
\end{aligned}
$$

e abrindo as somas, teremos :

$$
\begin{aligned}
R^{(e s p)} R^{(s p i n)} \varphi_{\tau} & =\frac{1}{\sqrt{2}}\left\{\varphi_{\tau}^{(1)} \chi_{1}\left[D_{11}^{(m)}(R) D_{12}^{(m)}(R)-D_{12}^{(m)}(R) D_{11}^{(m)}(R)\right]+\right. \\
& +\varphi_{\tau}^{(2)} \chi_{2}\left[D_{21}^{(m)}(R) D_{22}^{(m)}(R)-D_{22}^{(m)}(R) D_{21}^{(m)}(R)\right]+ \\
& \left.+\left(\varphi_{\tau}^{(1)} \chi_{2}-\varphi_{\tau}^{(2)} \chi_{1}\right)\left[D_{11}^{(m)}(R) D_{22}^{(m)}(R)-D_{12}^{(m)}(R) D_{21}^{(m)}(R)\right]\right\}
\end{aligned}
$$

e como os termos cruzados são nulos, ficamos somente com :

$$
R^{(e s p)} R^{(s p i n)} \varphi_{\tau}=\frac{1}{\sqrt{2}}\left(\varphi_{\tau}^{(1)} \chi_{2}-\psi_{\tau}^{(2)} \chi_{1}\right)\left[D_{11}^{(m)}(R) D_{22}^{(m)}(R)-D_{12}^{(m)}(R) D_{22}^{(m)}(R)\right]
$$


ou melhor :

$$
R^{(e s p)} R^{(s p i n)} \varphi_{\tau}=\|D(R)\| \varphi_{\tau}
$$

onde $\|D(R)\|$ é o determinante da matriz $D(R)$, o qual é -1 para as permutações ímpares $\{(12),(13),(23)\}$ e é $+\mathbf{1}$ para as permutações pares $\{(123) \equiv(12)(13),(132) \equiv(23)(12)\}$, como deveria ser para duas trocas sucessivas. E assim sendo temos a anti-simetria requerida. 


\section{Capítulo 7}

\section{Equação Radial}

Neste capítulo iremos deduzir a forma da nossa equação radial, mas primeiramente analisaremos o cálculo dos elementos de matriz que estão envolvidos no cálculo das curvas de potencial. Usaremos agora algumas propriedades das funções de canal advindas da simetrização imposta a esta e que residem na teoria dos operadores de projeção, seção 4.6.

\subsection{Cálculo das Curvas de Potencial}

O cálculo das curvas de potencial é dado a partir da seguinte equação para o potencial :

$$
\hat{\mathbf{U}}(R ; \Omega) \Phi_{n}(R ; \Omega)=U_{n}(R) \Phi_{n}(R: \Omega)
$$

sendo a função de canal dada por :

$$
\Phi(R ; \Omega)=\sum_{\tau^{\prime}} \Delta_{n}^{\tau^{\prime}}(R) \varphi_{\tau^{\prime}}(\Omega)
$$

e a sua base, anti-simétrica :

$$
\varphi_{\tau}=\frac{1}{\sqrt{2}}\left(\varphi_{\tau}^{(1)} \chi_{2}-\varphi_{\tau}^{(2)} \chi_{1}\right)
$$

onde $\tau$ é o conjunto de índices que rotulam a nossa função de base. Os elementos de matriz são obtidos ao projetarmos a equação (7.2) num estado $\varphi_{\tau}$ :

$$
\sum_{\tau^{\prime}}\left\langle\varphi_{\tau}|\hat{U}| \varphi_{\tau^{\prime}}\right\rangle \Delta_{n}^{\tau^{\prime}}(R)=U_{n}(R) \Delta_{n}^{\tau}(R)
$$

o que implica então no cálculo do seguinte elemento de matriz :

$$
\left\langle\varphi_{\tau}|\hat{U}| \varphi_{\tau^{\prime}}\right\rangle
$$


Devido à independência do hamiltoniano em relação aos spins, e a ortonormalidade das funções de spin, conseguimos reescrever o elemento de matriz da seguinte forma :

$$
\left\langle\varphi_{\tau}|\hat{U}| \varphi_{\tau^{\prime}}\right\rangle=\frac{1}{2}\left\{\left\langle\varphi_{\tau}^{(1)}|\hat{U}| \varphi_{\tau^{\prime}}^{(1)}\right\rangle+\left\langle\varphi_{\tau}^{(2)}|\hat{U}| \varphi_{\tau^{\prime}}^{(2)}\right\rangle\right\}
$$

sendo $\varphi_{\tau}$ dado como anteriormente. As funções $\varphi_{\tau}^{(1)}$ e $\varphi_{\tau}^{(2)}$ são dadas em função dos operadores de projeção :

$$
\begin{aligned}
& \varphi_{\tau}^{(1)}=\rho_{11}^{(m)} \phi_{\mu \nu l_{1} l_{2} l_{3} l_{12}}^{L M} \\
& \varphi_{\tau}^{(2)}=\rho_{21}^{(m)} \phi_{\mu \nu l_{1} l_{2} l_{3} l_{12}}^{L M I}
\end{aligned}
$$

os quais seguem as propriedades abaixo :

$$
\rho_{m \mu}^{(i)} \rho_{\nu n}^{(j)}=\delta_{i j} \delta_{\mu \nu} \rho_{m n}^{(j)}
$$

e

$$
\left(\rho_{\mu \nu}^{(j)}\right)^{\dagger}=\rho_{\nu \mu}^{(j)}
$$

demonstradas na seção $\mathbf{4 . 6}$.

A partir disto podemos realizar uma importante simplificação no cálculo dos elementos de matriz do nosso problema. Sendo então :

$$
\begin{gathered}
\rho_{21}^{(m)} \varphi_{\tau}^{(1)}=\varphi_{\tau}^{(2)}, \rho_{12}^{(m)} \varphi_{\tau}^{(2)}=\varphi_{\tau}^{(1)}, \\
\rho_{\mu \mu}^{(m)} \varphi_{\tau}^{\mu}=\varphi_{\tau}^{\mu} . \\
\rho_{12}^{(m)} \rho_{21}^{(m)}=\rho_{11}^{(m)}, \rho_{21}^{(m)} \rho_{12}^{(m)}=\rho_{22}^{(m)} .
\end{gathered}
$$

e a invariância do hamiltoniano por $\mathbf{S}_{\mathbf{3}}$, decorre que :

$$
\begin{aligned}
\left\langle\varphi_{\tau}^{(2)}|\hat{U}| \varphi_{\tau^{\prime}}^{(2)}\right\rangle & =\left\langle\rho_{21}^{(m)} \varphi_{\tau}^{(1)}|\hat{U}| \rho_{21}^{(m)} \varphi_{\tau^{\prime}}^{(1)}\right\rangle=\left\langle\varphi_{\tau}^{(1)}\left|\rho_{12}^{(m)} \hat{U}\right| \rho_{21}^{(m)} \varphi_{\tau^{\prime}}^{(1)}\right\rangle= \\
& =\left\langle\varphi_{\tau}^{(1)}|\hat{U}| \rho_{12}^{(m)} \rho_{21}^{(m)} \varphi_{\tau^{\prime}}^{(1)}\right\rangle=\left\langle\varphi_{\tau}^{(1)}|\hat{U}| \varphi_{\tau^{\prime}}^{(1)}\right\rangle \\
\left\langle\varphi_{\tau}^{(2)}|\hat{U}| \varphi_{\tau^{\prime}}^{(2)}\right\rangle & =\left\langle\varphi_{\tau}^{(1)}|\hat{U}| \varphi_{\tau^{\prime}}^{(1)}\right\rangle,
\end{aligned}
$$

de modo que assim o cálculo do nosso elemento (7.6) de matriz scria dado por:

$$
\begin{aligned}
\left\langle\varphi_{\tau}|\hat{U}| \varphi_{\tau^{\prime}}\right\rangle & =\left\langle\psi_{\tau}^{(2)}|\hat{U}| \varphi_{\tau^{\prime}}^{(2)}\right\rangle= \\
& =\left\langle\psi^{(1)}|\hat{U}| \psi_{\tau^{\prime}}{ }^{\prime}{ }^{\prime} .\right.
\end{aligned}
$$


assim nos dizendo que tendo em mãos $\varphi_{\tau}^{(1)}$ ou $\varphi_{\tau}^{(2)}$, só precisamos de uma das duas para realizar os cálculos para as curvas de potencial. Ainda temos que

$$
\begin{aligned}
\left\langle\varphi_{\tau}^{(1)}|\hat{U}| \varphi_{\tau^{\prime}}^{(1)}\right\rangle & =\left\langle\rho_{11}^{(m)} \phi_{\mu \nu l_{1} l_{2} l_{3} l_{12}}^{L M}|\hat{\mathbf{U}}| \varphi_{\tau}^{(1)}\right\rangle \\
& =\left\langle\phi_{\mu \nu l_{1} l_{2} l_{3} l_{12}}^{L M}|\hat{\mathbf{U}}| \rho_{11}^{(m)} \varphi_{\tau}^{(1)}\right\rangle \\
& =\left\langle\phi_{\mu \nu l_{1} l_{2} l_{3} l_{12}}^{L M}|\hat{\mathbf{U}}| \varphi_{\tau}^{(1)}\right\rangle,
\end{aligned}
$$

o que simplifica o cálculo dos elementos de matriz. Logo, o elemento de matriz é dado por :

$$
U_{\tau \tau^{\prime}}(R ; \Omega)=\left\langle\varphi_{\tau}|\hat{U}| \varphi_{\tau^{\prime}}\right\rangle=\left\langle\varphi_{\tau}^{(1)}|\hat{\mathbf{U}}(0)-2 R \hat{\mathbf{C}}| \varphi_{\tau^{\prime}}^{(1)}\right\rangle,
$$

e sendo a função $\varphi_{\tau}^{(1)}$ uma auto função do Casimir $\hat{\Lambda}_{9}^{2}$ teremos, de acordo com a equação (2.10) :

$$
\begin{aligned}
U_{\tau \tau^{\prime}}(R ; \Omega) & =U(0) \delta_{\tau \tau^{\prime}}-2 R\left\langle\varphi_{\tau}^{(1)}|\hat{\mathbf{C}}| \varphi_{\tau^{\prime}}^{(1)}\right\rangle \\
& =U(0) \delta_{\tau \tau^{\prime}}-2 R C_{\tau \tau^{\prime}},
\end{aligned}
$$

de maneira que o elemento de matriz $C_{\tau \tau^{\prime}}$ é uma combinação linear dos elementos de matriz $C_{\mu \nu l_{i} \mu^{\prime} \nu^{\prime} l_{i}^{\prime}}^{L M M}$ dados pela equação $(2.51)$, já que as funções projetadas são combinação linear das não projetadas. De forma assim termos agora uma matriz de representação para o operador $\hat{\mathbf{U}}(R ; \Omega)$, a qual se diagonalizada nos dará como auto-valores as curvas de potencial $U_{n}(R)$.

\subsection{Equação Radial, Acoplamentos $P_{\mu \nu}$ e $Q_{\mu \nu}$}

Tendo então em mãos as curvas de potencial $U_{n}(R)$ podemos agora analisar a equação radial a partir de :

$$
\left[\frac{d^{2}}{d R^{2}}+\frac{\hat{\mathbf{U}}(R ; \Omega)+1 / 4}{R^{2}}+2 E\right] \Psi(R ; \Omega)=0,
$$

onde :

$$
\Psi(R ; \Omega)=\sum_{\nu} F_{\nu}(R) \Phi_{\nu}(R ; \Omega),
$$

onde as funções de canal satisfazem a equação de auto-valores :

$$
\hat{\mathbf{U}}(R ; \Omega) \Phi_{\nu}(R ; \Omega)=U_{\nu}(R) \Phi_{\nu}(R ; \Omega) .
$$

Agora substituindo (7.16) em (7.15) teremos:

$$
\begin{gathered}
\sum_{\nu}\left[\Phi_{\nu} \frac{d^{2}}{d R^{2}}+2 \frac{d}{d R} \Phi_{\nu} \frac{d}{d R}+\frac{d^{2}}{d R^{2}} \Phi_{\nu}+\frac{U_{\nu}(R)+1 / 4}{R^{2}} \Phi_{\nu}+2 E \Phi_{\nu}\right] F_{\nu}(R)=0 \\
\| S 3
\end{gathered}
$$


na qual se projetarmos em $\Phi_{\mu}$, obteremos como resultado :

$$
\left[\frac{d^{2}}{d R^{2}}+\frac{U_{\mu}(R)+1 / 4}{R^{2}}+2 E\right] F_{\mu}(R)+\sum_{\nu}\left[2 P_{\mu \nu} \frac{d}{d R}+Q_{\mu \nu}\right] F_{\nu}(R)=0
$$

onde :

$$
P_{\mu \nu}(R) \equiv\left\langle\Phi_{\mu}\left|\frac{d}{d R}\right| \Phi_{\nu}\right\rangle=-\frac{\left\langle\Phi_{\mu}|\hat{\mathbf{C}}| \Phi_{\nu}\right\rangle}{U_{\mu}-U_{\nu}}
$$

e

$$
Q_{\mu \nu}(R) \equiv\left\langle\Phi_{\mu}\left|\frac{d^{2}}{d R^{2}}\right| \Phi_{\nu}\right\rangle=\frac{2}{U_{\mu}-U_{\nu}}\left[\frac{d}{d R} U_{\nu} P_{\mu \nu}-\sum_{\tau}\left\langle\Phi_{\mu}|\hat{\mathbf{C}}| \Phi_{\tau}\right\rangle\left\langle\Phi_{\tau}\left|\frac{d}{d R}\right| \Phi_{\nu}\right\rangle\right]
$$

são os termos de acoplamento não adiabático, de acordo com [25]. Agora podemos classificar o problema com relação aos acoplamentos $P_{\mu \nu}$ e $Q_{\mu \nu}$, da seguinte forma :

- Aproximação Adiabática Extrema (EAA) . Consiste em desprezar completamente os acoplamentos não adiabáticos :

$$
\begin{array}{ll}
P_{\mu \nu}=0 & \\
Q_{\mu \nu}=0 & \forall \mu, \nu,
\end{array}
$$

de modo a ficarmos com equação radial abaixo :

$$
\left[\frac{d^{2}}{d R^{2}}+\frac{U_{\mu}(R)+1 / 4}{R^{2}}+2 E\right] F_{\mu}(R)=0
$$

gerando um sistema desacoplado.

- Aproximação Adiabática Desacoplada (UAA). Consideramos neste caso somente os termos não adiabáticos diagonais :

$$
\begin{aligned}
& P_{\mu \nu}=0 \\
& Q_{\mu \nu}=0 \quad \mu \neq \nu,
\end{aligned}
$$

nos dando a equação radial :

$$
\left[\frac{d^{2}}{d R^{2}}+\frac{U_{\mu}(R)+1 / 4}{R^{2}}+2 E+2 P_{\mu \mu} \frac{d}{d R}+Q_{\mu \mu}\right] F_{\mu}(R)=0
$$

que também forma um sistema desacoplado.

- Aproximação Adiabática Acoplada (CAA) Consiste em considerar todos os termos não adiabáticos :

$$
\begin{aligned}
& P_{\mu \nu} \neq 0 \\
& Q_{\mu \nu} \neq 0 \quad \forall \mu, \nu .
\end{aligned}
$$


nos dando o sistema de equações acopladas :

$$
\left[\frac{d^{2}}{d R^{2}}+\frac{U_{\mu}(R)+1 / 4}{R^{2}}+2 E\right] F_{\mu}(R)+\sum_{\nu}\left[2 P_{\mu \nu} \frac{d}{d R}+Q_{\mu \nu}\right] F_{\nu}(R)=0,
$$

As energias obtidas para cada uma destas aproximações ( $E_{E A A}, E_{U A A}$ e $\left.E_{C A A}\right)$ se dispõem numa desigualdade denominada 'Basic Inequalities' :

$$
E_{E A A} \leq E \leq E_{C A A} \leq E_{U A A}
$$

demonstrada no trabalho "Proof of Basic Inequalities in the Hyperspherical Formalism for N-Body Problem", [9], nos dizendo que $E_{E A A}$ é um limite inferior e $E_{C A A}$ e $E_{U A A}$ limites superiores, de forma assim podemos 'cercar' o valor pretendido, $E$, conforme discutido na introdução. 


\section{Capítulo 8}

\section{Resultados e Conclusões}

Neste trabalho focalizamos o estudo do átomo de lítio dentro dos limites da aproximação adiabática extrema, na qual descartamos os efeitos de quaisquer correções não adiabáticas, $P_{\mu \nu}$ e $Q_{\mu \nu}$, estabelecendo assim a seguinte equação hiperesférica radial

$$
\left[\frac{d^{2}}{d R^{2}}+\frac{U_{\mu}(R)+1 / 4}{R^{2}}+2 E_{E A A}\right] F_{\mu}(R)=0
$$

$\mathrm{Na}$ aproximação adiabática extrema cada curva de potencial $U_{\mu}(R)$ determina uma equação de movimento na coordenada radial, equação (8.1). As soluções de cada uma das equações de movimento representam então auto-estados para o sistema de três elétrons com níveis discretos e contínuos.

O processo de obtenção dos resultados consiste em primeiramente determinar-se uma base para o conjunto de funções de canal, a partir da simetrização apropriada dos hiperesféricos harmônicos do $S O(9)$, via os operadores de projeção da simetria de permutação, e de sua ortogonalização pelo método de Gram-Schmidt, conforme discutido nos capítulos anteriores. Tendo em mãos a base para as funções de canal o próximo passo é calcular os elementos de matriz da energia potencial, (7.11), e a partir da sua diagonalização determinar-se o conjunto de curvas de potencial para o nosso problema, possibilitando assim um primeiro cálculo para a energia adiabática extrema. 


\subsection{Funções Simetrizadas para $L=0$}

Geramos os hiperesféricos harmônicos do $S O(9)$ com as seguintes regras de seleção

$$
\begin{aligned}
& 2 \mu+2 \nu+l_{1}+l_{2}+l_{3}=\lambda_{9}, \\
& \left|l_{1}-l_{2}\right| \leq l_{12} \leq l_{1}+l_{2}, \\
& \left|l_{3}-l_{12}\right| \leq L \leq l_{3}+l_{12}, \\
& (-1)^{l_{1}+l_{2}+l_{3}}=1, \\
& m_{1}+m_{2}=m_{12}, \\
& m_{4}+m_{3}=M .
\end{aligned}
$$

Ao projetarmos as funções de um determinado setor $\lambda_{9}$, o resultado muitas vezes se repete, isto é, a projeção de funções diferentes pode gerar um mesmo resultado, fazendo com que o número de funções simetrizadas, linearmente independente, no setor projetado diminua.

Esta redução no número de funções por setor, deve-se essencialmente ao fato de os hiperesféricos conterem naturalmente uma certa simetria de permutação. Isto pode ser visto considerando a sua forma em coordenadas esféricas

$$
\begin{aligned}
\phi_{\mu \nu l_{1} l_{2} l_{3} l_{12}}^{L M}\left(r_{1}, r_{2}, r_{3}\right)= & N_{\mu}^{l_{1} l_{2}} N_{\mu \nu}^{l_{1} l_{2} l_{3}} r_{1}^{l_{1}} r_{2}^{l_{2}} r_{3}^{l_{3}}\left(r_{1}^{2}+r_{2}^{2}\right)^{\mu} R^{2 \nu} P_{\mu}^{\left(l_{1}+1 / 2, l_{2}+1 / 2\right)}\left(\frac{r_{2}^{2}-r_{1}^{2}}{r_{1}^{2}+r_{2}^{2}}\right) \\
& P_{\nu}^{\left(2 \mu+l_{1}+l_{2}+2, l_{3}+1 / 2\right)}\left(\frac{r_{3}^{2}-r_{2}^{2}-r_{1}^{2}}{R^{2}}\right) \mathcal{Y}_{l_{1} l_{2} l_{3} l_{12}}^{L M}\left(\Lambda_{1}, \Lambda_{2}, \Lambda_{3}\right)
\end{aligned}
$$

O operador de projeção pode ser reescrito de uma forma muito conveniente

$$
\rho_{11}^{(m)}=\frac{1}{6}[2(12)-(13)-(23)][e+(12)]
$$

sendo a atuação da permutação (12) nos hiperesféricos dada por

$$
\text { (12) } \phi_{\mu \nu l_{1} l_{2} l_{3} l_{12}}^{L M}=(-1)^{\mu+l_{1}+l_{2}+l_{12}} \phi_{\mu \nu l_{2} l_{1} l_{3} l_{12}}^{L M}
$$

de acordo com o discutido nos capítulos anteriores. Deste modo ao atuarmos o operador $\rho_{11}^{(m)}$ nos hiperesféricos obteremos 


$$
\rho_{11}^{(m)} \phi_{\mu \nu l_{1} l_{2} l_{3} l_{12}}^{L M}=\frac{1}{6}[2(12)-(13)-(23)]\left[\phi_{\mu \nu l_{1} l_{2} l_{3} l_{12}}^{L M}+(-1)^{\mu+l_{1}+l_{2}+l_{12}} \phi_{\mu \nu l_{2} l_{1} l_{3} l_{12}}^{L M}\right]
$$

Para o estado fundamental ( $L=0$ ), o qual é de nosso interesse, teremos $l_{12}=l_{3} \mathrm{e}$ $(-1)^{l_{1}+l_{2}+l_{3}}=1$ simplificando a atuação de $\rho_{11}^{(m)}$

$$
\rho_{11}^{(m)} \phi_{\mu \nu l_{1} l_{2} l_{3}}=\frac{1}{6}[2(12)-(13)-(23)]\left[\phi_{\mu \nu l_{1} l_{2} l_{3}}+(-1)^{\mu} \phi_{\mu \nu l_{2} l_{1} l_{3}}\right],
$$

onde podermos ver que para os hiperesféricos com $\mu$ ímpar e $l_{1}=l_{2}$ a projeção será nula. As projeções $\rho_{11}^{(m)} \phi_{\mu \nu l_{1} l_{2} l_{3}}$ e $\rho_{11}^{(m)} \phi_{\mu \nu l_{2} l_{1} l_{3}}$ dão o mesmo resultado, a menos de uma fase $(-1)^{\mu}$. Desta maneira vemos claramente o porque da redução na dimensão dos setores $\lambda_{9}$.

Colocamos abaixo a tabela com a redução para cada um dos setores utilizados ao se aplicar o projetor $\rho_{11}^{(m)}$, omitindo na notação os valores de $L=0, M=0$ e $l_{12}=l_{3}$

\begin{tabular}{ccc}
\hline \hline $\begin{array}{c}\text { setor } \\
\lambda_{9}\end{array}$ & $\begin{array}{c}\text { n. de hiperesféricos } \\
\left(\phi_{\mu \nu l_{1} l_{2} l_{3}}\right)\end{array}$ & $\begin{array}{c}\text { n. de projeções l.i. } \\
\left(\varphi_{\tau}=\rho_{11}^{(m)} \phi_{\mu \nu l_{1} l_{2} l_{3}}\right)\end{array}$ \\
\hline 2 & 5 & 2 \\
4 & 15 & 5 \\
6 & 35 & 11 \\
8 & 70 & 24 \\
\hline
\end{tabular}

Tabela 8.1: Dimensão dos setores $\lambda_{9}=2,4,6$ e 8 , para $L=0$.

o que totaliza por final em 42 funções de canal.

\subsection{Curvas de Potencial}

O cálculo dos elementos de matriz para as interações coulombianas foi efetuado analiticamente através da implementação de rotinas em maple, de modo a podermos utilizar estes resultados na estratégia de debuging, ao implementarmos os códigos em fortran, tornando possível expandir a dimensão dos nossos cálculos.

O cálculo das curvas de potencial é feito a partir da diagonalização do operador $\hat{\mathbf{U}}(R)=$ $\hat{\mathbf{U}}(0)-2 R \hat{\mathbf{C}}(\Omega)$ para valores fixos da coordenada radial $R$. Este cálculo é feito utilizando-st. 
rotinas em fortran, as mesmas utilizadas para os cálculos precisos para o átomo de hélio [16], tomando um espaçamento de pontos de $\Delta R=0.01$.

Neste tabalho tratamos as curvas de potencial para a simetria $S=1 / 2$ (dubleto) nos spins. Utilizamos os hiperesféricos harmônicos simetrizados para uma configuração de momento angular $\left(l_{1}, l_{2}, l_{3}\right)$ arbitrária, isto é, para todos os valores possíveis de $\left(l_{1}, l_{2}, l_{3}\right)$, com $\lambda_{9}$ até 8. Posteriormente adicionamos hiperesféricos na configuração $(0,0,0)$, com $\lambda_{9}$ de 10 até 28 . Esquematizamos abaixo a estrutura de cada um dos setores $\lambda_{9}$ utilizados neste trabalho

\begin{tabular}{|c|c|c|c|c|c|}
\hline$\lambda_{y}$ & $\begin{array}{l}\text { número } \\
\text { de canais }\end{array}$ & $\begin{array}{l}\text { configuração } \\
(0,0,0) \\
\end{array}$ & $\begin{array}{l}\text { configuração } \\
(l, l, l)\end{array}$ & $\begin{array}{l}\text { configuração } \\
\left(l, l, l^{\prime}\right)\end{array}$ & $\begin{array}{l}\text { configuração } \\
\left(l, l^{\prime}, l^{\prime \prime}\right) \\
\end{array}$ \\
\hline 2 & 2 & 1 & 0 & 1 & 0 \\
\hline 4 & 5 & 1 & 0 & 4 & 0 \\
\hline 6 & 11 & 1 & 0 & 8 & 2 \\
\hline 8 & 24 & 2 & 1 & 15 & 6 \\
\hline 10 & 2 & 2 & - & - & - \\
\hline 12 & 2 & 2 & - & - & - \\
\hline 14 & 3 & 3 & - & - & - \\
\hline 16 & 3 & 3 & - & - & - \\
\hline 18 & 3 & 3 & - & - & - \\
\hline 20 & 4 & 4 & - & - & - \\
\hline 22 & 4 & 4 & - & - & - \\
\hline 24 & 4 & 4 & - & - & - \\
\hline 26 & 5 & 5 & - & - & - \\
\hline 28 & 5 & 5 & - & - & - \\
\hline $\begin{array}{l}\text { número total } \\
\text { de canais }\end{array}$ & 77 & & & & \\
\hline
\end{tabular}

Tabela 8.2: Número de funções de canal para o átomo de lítio para $L=0$ e $S=1 / 2$, até $\lambda_{9}=28$.

Temos no total de 40 hiperesféricos simetrizados na configuração $(0,0,0)$, para os quais obtemos o seguinte conjunto de curvas de potencial esboçado na figura 8.1. onde podemos notar claramente o padrão de degenerescência em $R=0$, devido a forma do auto-valor dá energial potencial.

A adição das outras 37 funções, de configuração de momento angular arbitrária não altera substâncialmente a profundidade das curvas de potencial. mas porém podemos notar surpreendentemente o surgimento de novos avoid crossings, os quais são resultantes da regra de não cruzamento entre de potenciais adiabáticos. e que podem ser observados na figura 8.2 , a seguir. 

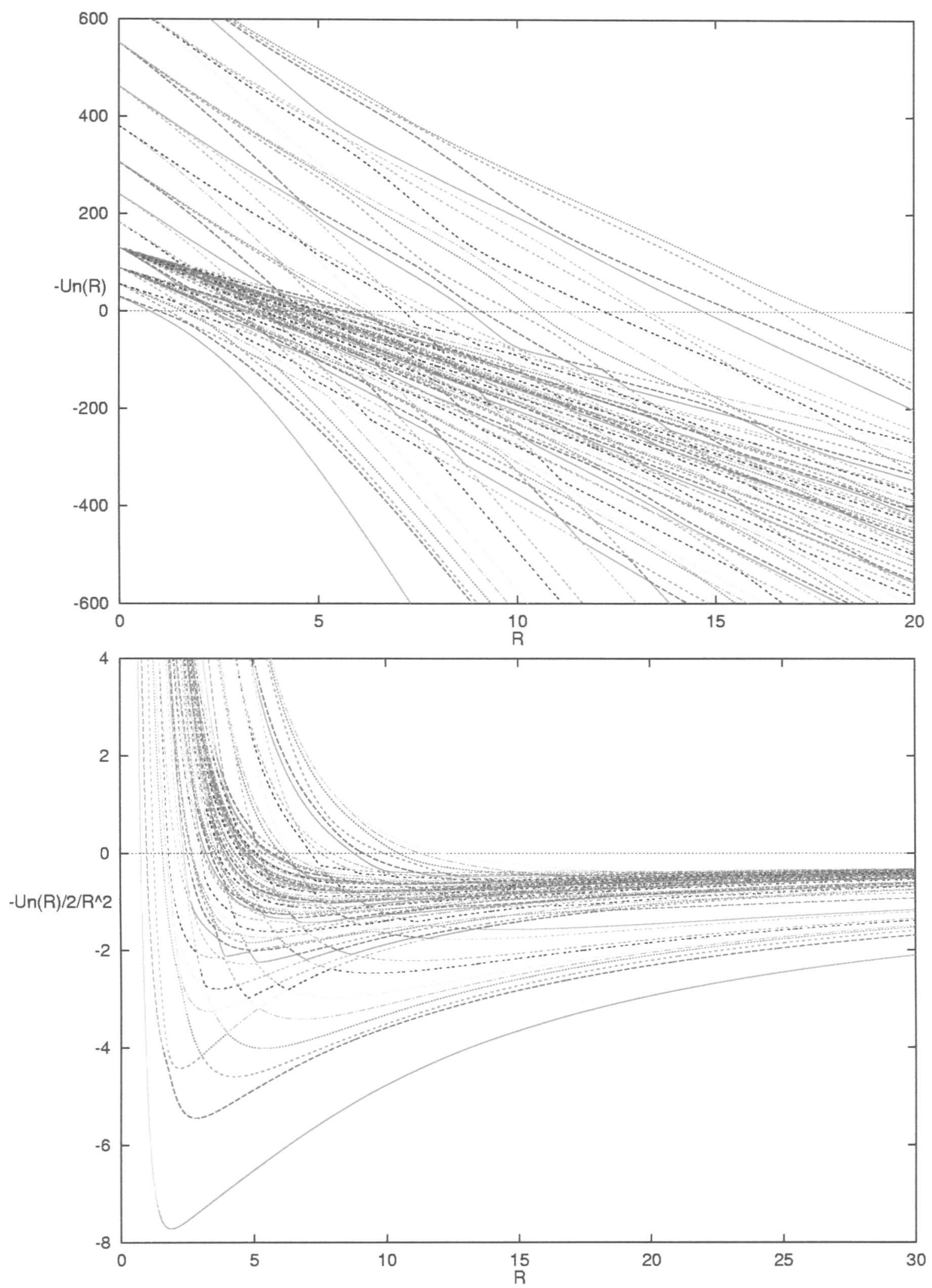

Figura 8.2: Curvas de Potencial $-U_{\mu}(R)$ e $-U_{\mu}(R) / 2 R^{2}$ para o átomo de lítio com $\lambda_{9}$ até 28 , adicionando as funções de momento angular orbital arbitrário. 

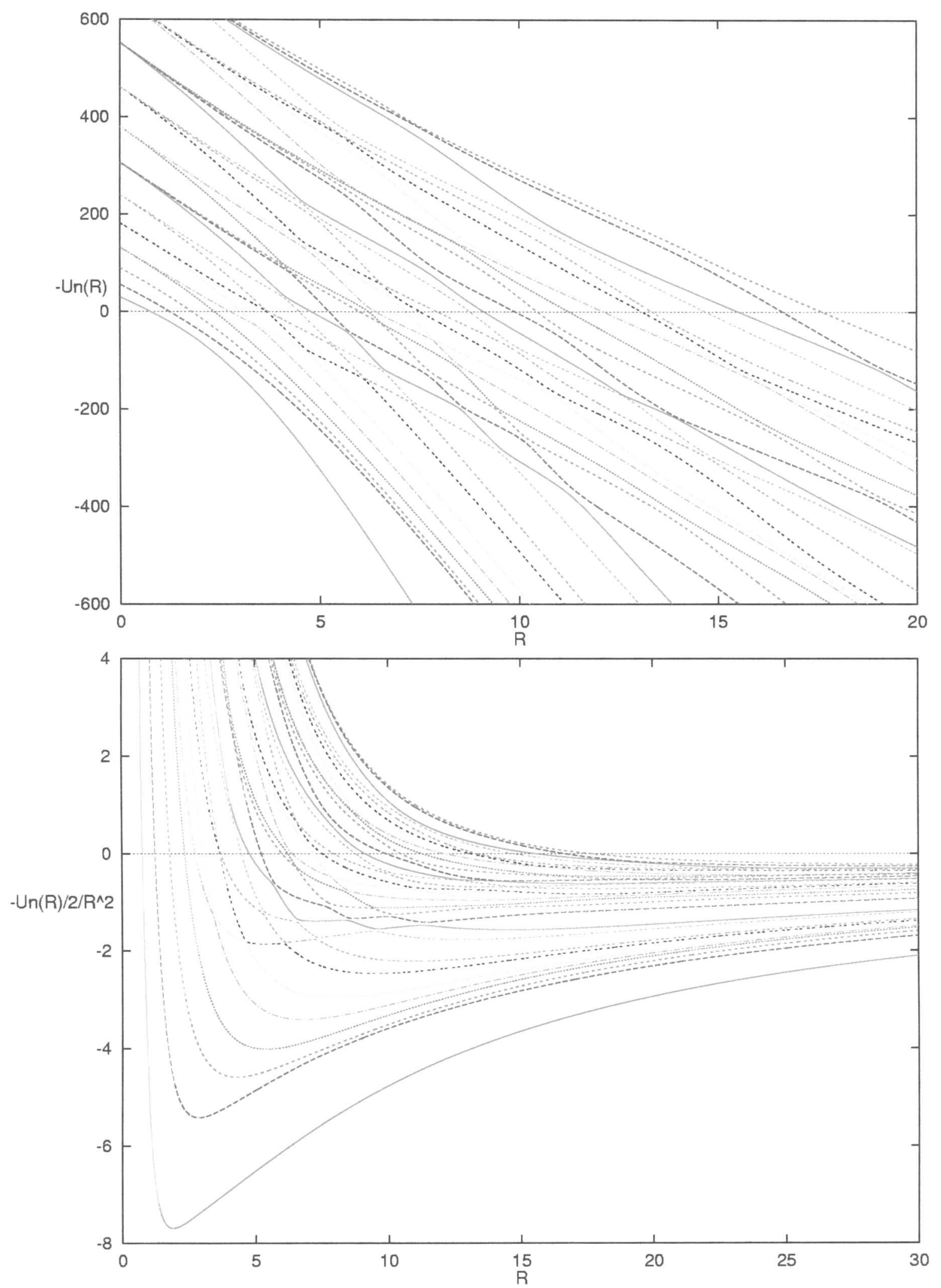

Figura 8.1: Curvas de Potencial $-U_{\mu}(R)$ e $-U_{\mu}(R) / 2 R^{2}$ para o átomo de lítio, na configuração $(0,0,0)$ com $\lambda_{9}$ até 28 . 
As curvas de potencial podem serem vistas como a "evolução" de um auto-estado de acordo com o parâmetro $R$. Na região dos avoid crossings, figuras $\mathbf{8 . 3}$ e $\mathbf{8 . 4}$, a função de canal irá mudar rapidamente e portanto os acoplamentos não adiabáticos $P_{\mu \nu}(R)$ e $Q_{\mu \nu}(R)$, entre diferentes canais serão extremamente importantes, exibindo um valor significativo nesta região, como pode-se ver claramente para os coeficiente $P_{\mu \nu}(R)$

$$
P_{\mu \nu}(R)=-\frac{\left\langle\Phi_{\mu}|\hat{\mathbf{C}}| \Phi_{\nu}\right\rangle}{U_{\mu}(R)-U_{\nu}(R)}
$$

Portanto, o interação entre canais próximos à região de avoid crossing é de fundamental importância na precisão do cálculo da energia nas aproximações adiabática desacoplada e acoplada ( $E_{U A A}$ e $\left.E_{C A A}\right)$.

Devemos ainda notar o comportamento assintótico das curvas de potencial. É de se esperar que a curva mais baixa vá convirgir assintóticamente para o valor da primeira energia de ionização, isto é, para $E_{L^{+}{ }^{+}}^{(v a r .)}=-7.279913386$ a.u., [30]. A nossa melhor curva, com 77 canais, cruza este limite em aproximadamente $R \simeq 4$.
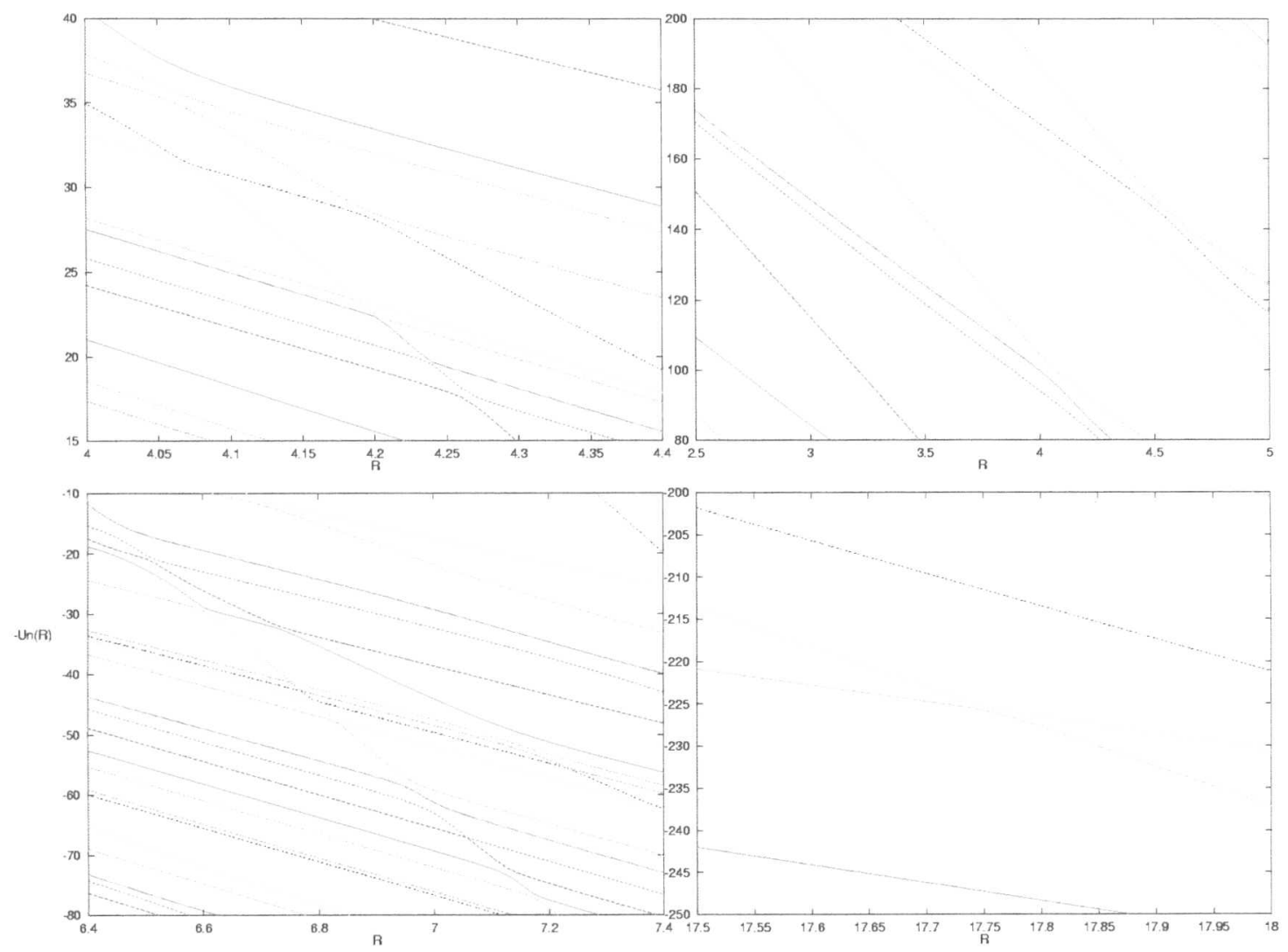

Figura 8.3: Curvas de potencial na região dos anti-crossings, figura 8.2.(1). 

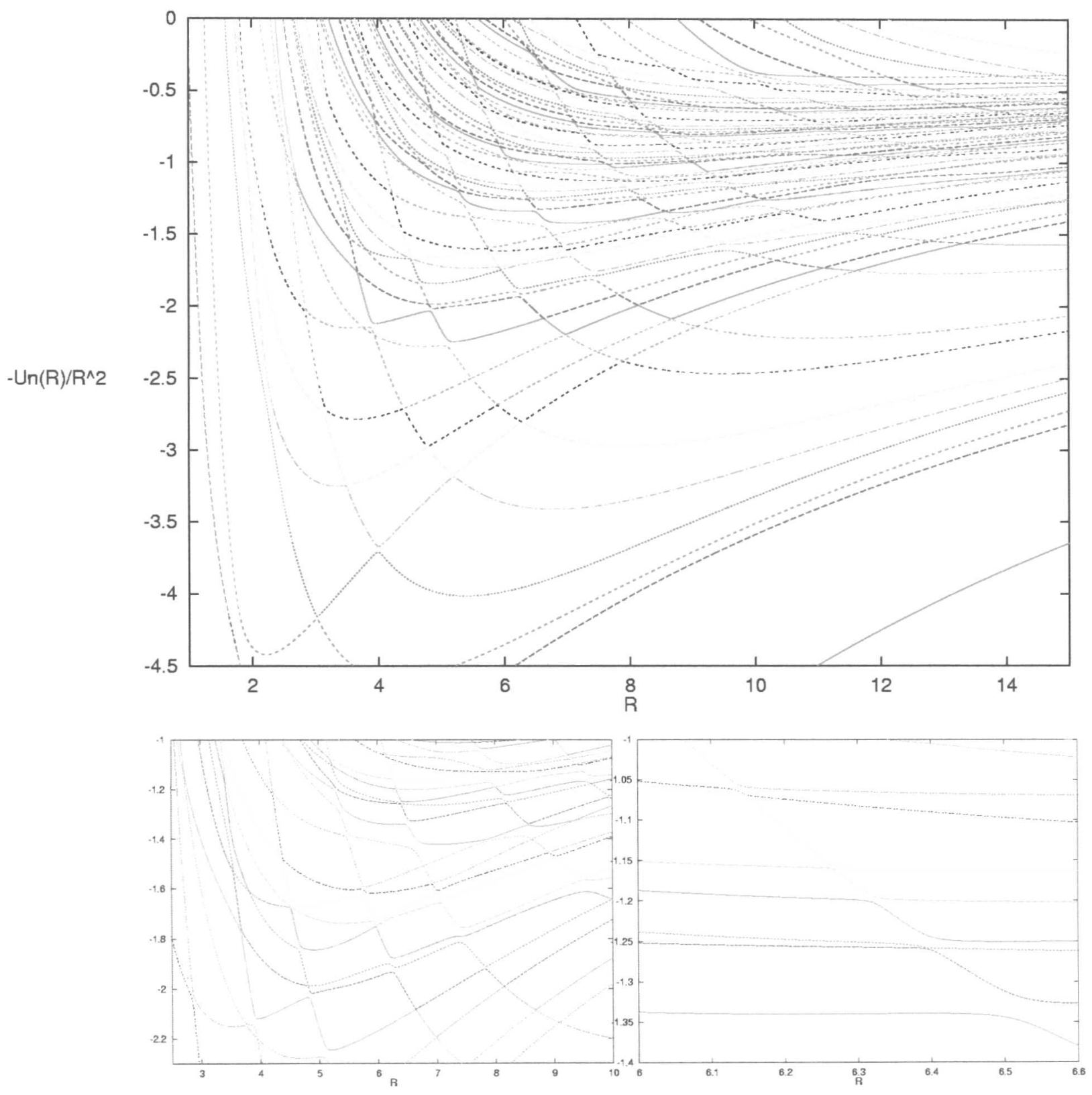

Figura 8.4: Curvas de potencial na região dos anti-crossings, figura 8.2.(2). 


\subsection{Energia Adiabática Extrema, $E_{E A A}$}

$\mathrm{Na}$ aproximação adiabática extrema cada curva de potencial $U_{\mu}(R)$ determina uma equação de movimento na coordenada radial, equação (8.1). As soluções de cada uma das equações de movimento representam então auto-estados para o sistema de três elétrons com níveis discretos e contínuos.

O menor auto-estado obtido ao se resolver a equação radial hiperesférica utilizando a curva de potencial mais baixa, é o estado fundamental para o átomo de lítio, enquanto os outros auto-estados representam as suas excitações.

Para termos uma idéia de como o sistema se comporta em relação ao aumento do número de canais considerados, podemos estudar o comportamento da curva de potencial mais baixa em função dos canais. Para tal análise obtemos os resultados esboçados na figura 8.5, na próxima página.

Para cálcular a energia adiabática extrema, através da equação

$$
\left[\frac{d^{2}}{d R^{2}}+\frac{U_{\mu}(R)+1 / 4}{R^{2}}+2 E_{E A A}^{(0)}\right] F_{\mu}(R)=0 .
$$

utilizamos o método de Runge-Kutta de quarta ordem, tomando um intervalo de pontos de $\Delta R=0.01$, impondo a condição de anulamento da função radial para $R$ assintótico. O nosso melhor valor para a energia adiabática extrema é calculada na aproximação de 77 canais, gerando os resultados apresentados na tabela $\mathbf{8 . 3}$.

A energia adiabática extrema para o estado fundamental do átomo de lítio ainda não alcançou o limite estabelecido pelo teorema das desigualdades básicas, isto é, a energia tem um valor mais alto do que a energia variacional, devido ao baixo números de de canais. A função radial obtida a partir da curva de potencial mais baixa, pode ser vista na figura (8.6). 

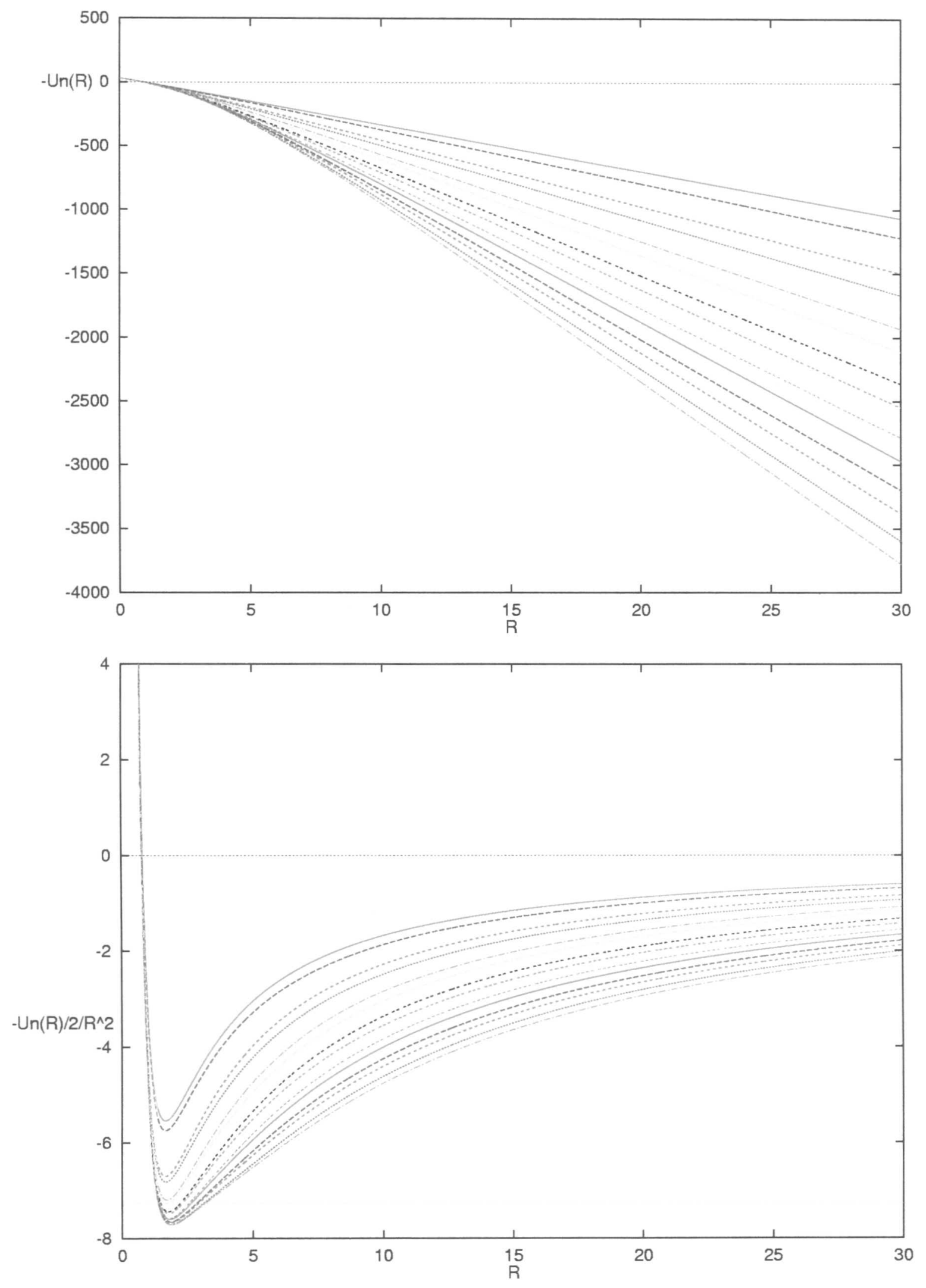

Figura 8.5: Convergência das curvas de potencial de acordo com o número de canais.

De cima para baixo temos curvas com 2, 7, 18, 42, 44, 46, 49, 52, 55, 59, 63, 67, 72 e 77 canais. 


\begin{tabular}{ccc}
\hline \hline n. canais & $E_{E A A}^{(0)}($ a.u. $)$ & $\left(E_{E A A}^{(0)}-E^{(\text {var. })}\right) / E^{(\text {var. })}$ \\
\hline 2 & -4.6550 & -0.3775 \\
7 & -4.8675 & -0.3491 \\
18 & -5.8007 & -0.2243 \\
42 & -5.9439 & -0.2052 \\
44 & -6.3759 & -0.1474 \\
46 & -6.4628 & -0.1358 \\
49 & -6.7052 & -0.1034 \\
52 & -6.7629 & -0.0956 \\
55 & -6.9127 & -0.0756 \\
59 & -6.9525 & -0.0703 \\
63 & -7.0518 & -0.0570 \\
67 & -7.0801 & -0.0532 \\
72 & -7.1493 & -0.0440 \\
77 & -7.169994 & -0.0412 \\
\hline$E^{(v a r)}=-7.478062$ a.u. & & \\
\hline \hline
\end{tabular}

Tabela 8.3: Energia do estado fundamental para o átomo de lítio na aproximação adiabática extrema 


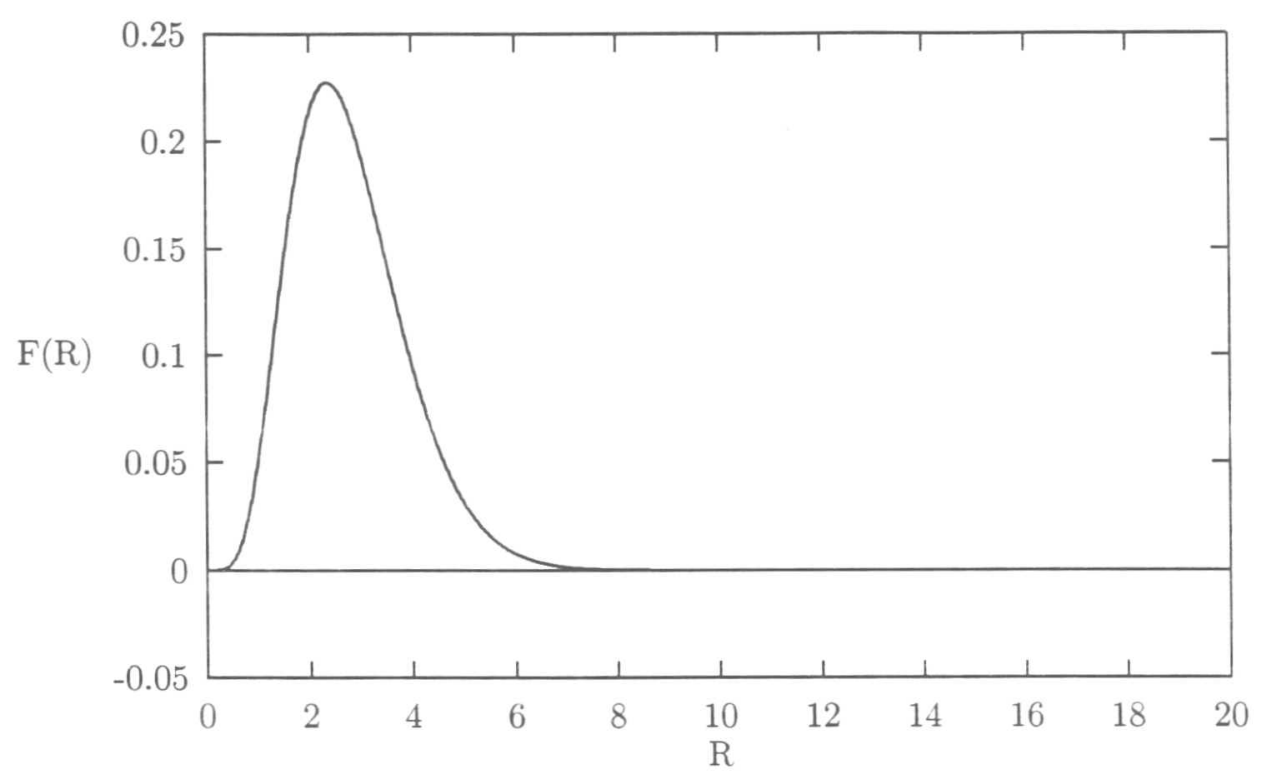

Figura 8.6: Função hiperesférica radial para o estado fundamental do átomo de lítio, a partir da curva de potencial mais baixa com 77 canais.

\subsection{Conclusões e Perspectivas}

A obtenção de funções de canal com a simetria de permutação definida, requisitada pelo princípio de exclusão de Pauli, é de fundamental importância para os sistemas atômicos. O processo de simetrização deve ser realizado a partir da análise das simetrias da parte spinorial, de modo a que possamos determinar qual a simetria deve ser imposta à parte espacial para que tenhamos uma função de onda total anti-simétrica.

Construimos aqui as bases para as funções de canal do átomo de lítio, com spin total $S=1 / 2$, a partir do uso dos hiperesféricos harmônicos do grupo $S O(9)$, impondo a estes a simetria mista [21] do grupo de permutação $S_{3}$ através do uso dos operadores de projeção. Pois, conforme demonstramos no capítulo 5, o produto de Kronecker de duas representações sendo que uma delas é a representação mista [21], no caso as funções de spin $1 / 2$, somente iremos obter uma representação totalmente anti-simétrica se a outra também portar a mesma representação mista. Para isto foi elaborado códigos em maple que geram as projeções dos hiperesféricos, calculadas analiticamente. Os resultados obtidos para atuação do operador de projeção $\rho_{11}^{(m)}$ nos quatro primeiros setores, $\lambda_{9}=2,4,6$ e 8, podem serem vistos no apêndice B. O uso dos operadores de projeção para a simetrização da base hiperesférica do sistema de N-elétrons é um tema profundo, tocando na relação existente entre o grupo de permutação 
$\mathbf{S}_{N}$ e o grupo de rotações $S O(3 N)$.

Para a analise das curvas de potencial consideramos, como um primeiro passo, o conjunto de curvas pertencentes à configuração de momento angular $(0,0,0)$, a qual inclui a curva de potencial mais baixa com números quânticos $0,1,0,0,0,\left(\mu, \nu, l_{1}, l_{2}, l_{3}\right)$, na origem, numa aproximação utilizando-se 40 canais ( $\lambda_{9}$ até 28 ). Para esta família de curvas de potencial encontramos na literatura o trabalho de C. H. Greene e C. W. Clark, [19], numa aproximação de 70 canais ( $\lambda_{9}$ até 38 ). Como podemos observar, a inclusão de canais mais altos, para esta configuração de momento angular, resultou uma melhor convergência para a curva mais baixa na região de grandes valores de $R$, porém para pequenos valores de $R$ as aproximações, de 40 e 70 canais, estão de acordo.

A inclusão de configurações de momento angular arbitrárias ( $\lambda_{9}$ até 8 ) não alterou significativamente a convergência para grandes valores de $R$. No entanto com a inclusão de curvas com configurações mais altas gerou o surgimento de novos avoid crossings, o que é um resultado novo, pois na literatura os cálculos tratam apenas de baixas configurações de momento angular, $(0,0,0)$ e $(1,1,0)$.

Observamos então que as funções de configuração $(0,0,0)$ são as que mais contribuem para a convergência da curva de potencial mais baixa, estando assim de completo acordo com resultados apresentados na literatura.

Devemos ainda notar o comportamento assintótico das curvas de potencial. É de se esperar que a curva mais baixa vá convirgir assintóticamente para o valor da primeira energia de ionização, isto é, para $E_{L^{+}{ }^{+}}^{(v a r)}=-7.279913386$ a.u., [30]. A nossa melhor curva, com 77 canais, cruza este limite em aproximadamente $R \simeq 4$.Isto seria de se esperar pois utilizamos como base para as funções de canal as auto-funções do potencial em $R=0$, e que obviamente apresentarão uma convergência mais lenta para valores grandes de $R$.

O método adiabático hiperesférico proporciona uma visão conceitual clara, descrevendo as propriedades dos sistemas em termos das curvas de potencial, de modo a poder ser usado com sucesso na descrição de sistemas de quatro corpos. Aqui o método foi aplicado em um sistema de forças coulombianas, de longo alcance, mas pode ser igualmente aplicado para forças de curto e médio alcance.

No entanto, afim de alcançarmos a precisão almejada, deparamos com o problema de se estabelecer o limite assintótico correto para as curvas de potencial. Este problema no entanto deverá ser tratado através da inclusão de funções de canal cuja a base encorpore também o 


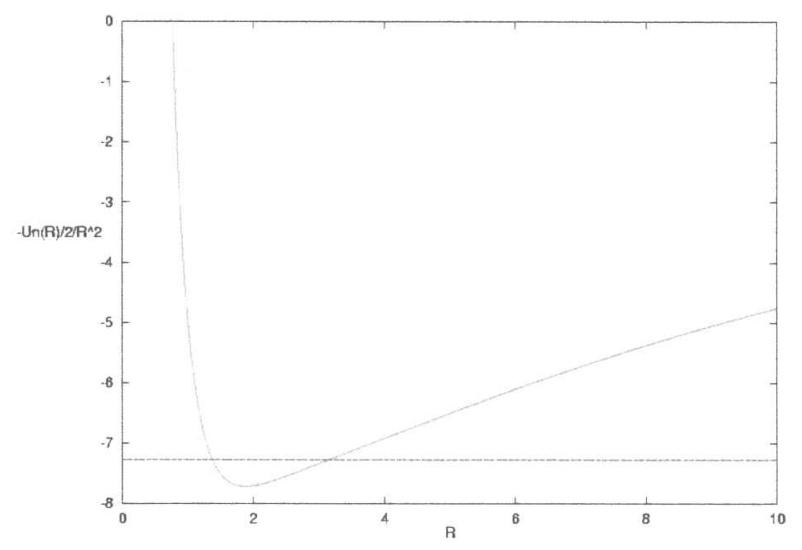

Figura 8.7: A curva de potencial mais baixa.

limite assintótico correto, isto é, uma base que se comporte bem nas proximidades de $R=0$ e para grandes valores de $R$.

Esta nova base deverá seguir as mesmas leis de simetria das funções aqui desenvolvidas, isto é, a simetria mista [21] do grupo de permutação $S_{3}$ deverá ser imposta da mesma maneira, de modo a obtermos uma função de onda totalmente anti-simétrica.

A investigação dos anti-crossings, [31] e [32], nas curvas de potencial é de fundamental importância, pois é nesta região que teremos uma grande contribuição dos coeficientes $P_{\mu \nu}(R)$ e $Q_{\mu \nu}(R)$ para a energia, como confirmam as experiências anteriores, [13] e [16]. Portanto a inclusão dos coeficientes não adiabáticos, $P_{\mu \nu}(R)$ e $Q_{\mu \nu}(R)$, juntamente com a inclusão de uma base que encorpore o limite assintótico correto, deve aumentar apreciavelmente a precisão dos nossos resultados, proporcionando assim valores competitivos perante à comunidade científica. 


\section{Apêndice A}

\section{Curvas de Potencial $\lambda_{9}=2$}

Faremos agora um exemplo simples do procedimento padrão adotado para se determinar as curvas de potencial para o átomo de Lítio. Para o setor $\lambda_{9}=2$ temos apenas duas funções de base :

$$
\begin{aligned}
\varphi_{1} & =\phi_{01000} \\
\varphi_{2} & =\frac{\sqrt{6}}{6} \phi_{00011}+\frac{\sqrt{6}}{6} \phi_{00101}-\frac{\sqrt{6}}{3} \phi_{00110}
\end{aligned}
$$

de modo que trabalharemos com matrizes $2 \times 2$. Com o programa em Maple calculamos os elementos de matriz para as interações Coulombianas, a matriz $\hat{\mathbf{C}}$, de forma a obtermos :

$$
\hat{\mathbf{C}}=\left(\begin{array}{ll}
-18.30412192 & -.1127929690 \\
-.1127929690 & -14.32333171
\end{array}\right) \text {. }
$$

A matriz $\hat{U}(0)$ é fácilmente determinada :

$$
U(0)=\left(\begin{array}{ll}
-30.25 & 0 \\
0 & -30.25
\end{array}\right)
$$

Sendo matriz para potencial dada por :

$$
\hat{\mathbf{U}}=\hat{\mathbf{U}}(0)-2 R \hat{\mathbf{C}}
$$

podemos então determina-la :

$$
\hat{\mathrm{U}}=\left(\begin{array}{cc}
-30.25+36.608 R & .22559 R \\
.22559 R & -30.25+28.647 R
\end{array}\right)
$$


e sabendo que as curvas de potencial são os auto-valores do operador $\hat{\mathbf{U}}$ :

$$
\hat{\mathbf{U}}(R ; \Omega) \Phi_{\nu}(R ; \Omega)=U_{\nu}(R) \Phi_{\nu}(R ; \Omega),
$$

a determinação das curvas de potencial implica em resolver o determinante, equação secular

$$
\left|\begin{array}{cc}
-30.25+36.608 R-U & .22559 R \\
.22559 R & -30.25+28.647 R-U
\end{array}\right|=0 .
$$

Para o caso bidimensional a solução é bem simples :

$$
915.1-1974.0 R+60.5 U+1048.7 R^{2}-65.3 R U(R)+U(R)^{2}=0,
$$

a qual foi calculada analíticamente utilizando-se do programa de computação algébrica Maple. Desta equação de segundo grau em $U(R)$, tiramos as curvas de potencial :

$$
\begin{aligned}
& U_{1}(R)=-30.25+36.6 R, \\
& U_{2}(R)=-30.25+28.6 R,
\end{aligned}
$$

solucionando então o problema. Tendo então uma curva de potencial, a qual é uma função de $R$, podemos resolver a equação radial, numéricamente e determinar o valor da energia. A extenção do problema para mais funções agora fica bem claro.

Plotamos abaixo as curva de potencial, auto-valores de $\hat{\mathbf{U}}$ para o caso bidimensional, (A.5) e (A.6) : 


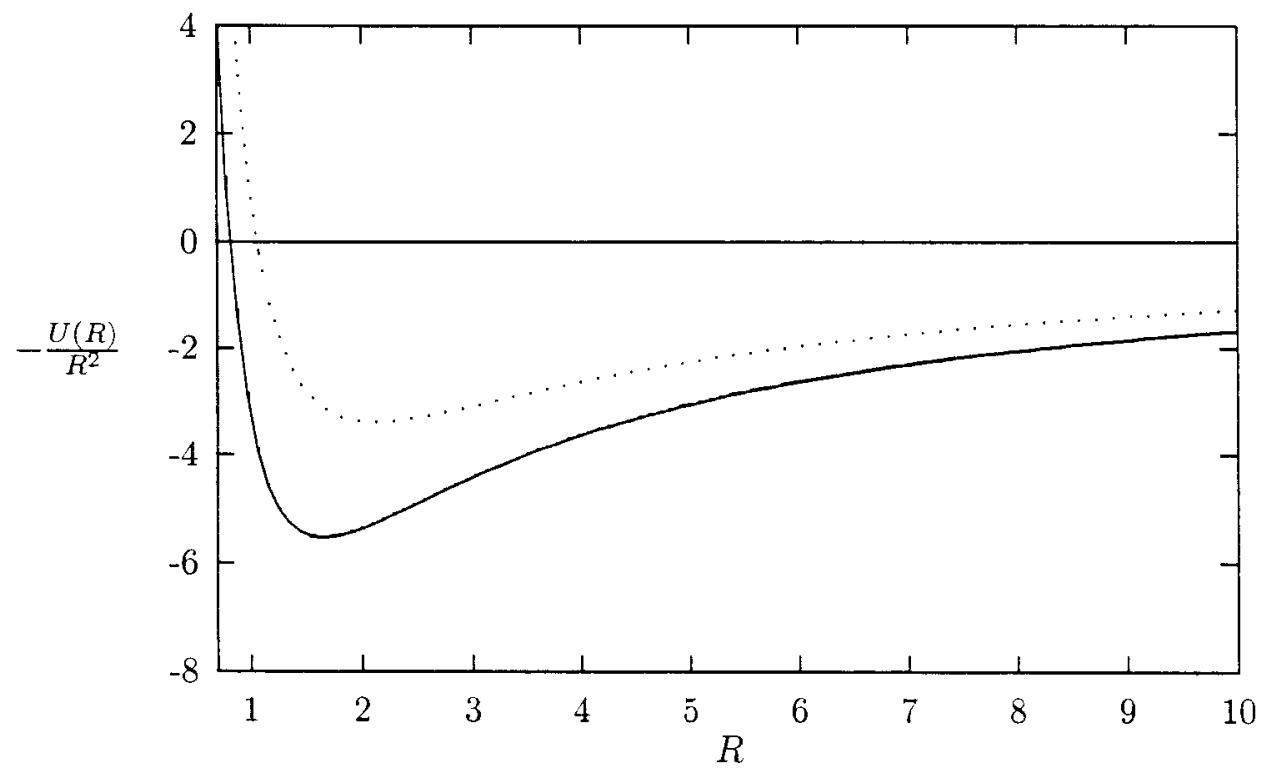

Figura A.1: Curvas de Potencial para a aproximação $\lambda_{9}=2$. 


\section{Apêndice B}

\section{Atuação de $\rho_{11}^{(m)}$ em $\lambda_{9}=2,4,6$ e 8}

Mostramos a seguir os resultados obtidos para o operador de projeção $\rho_{11}^{(m)}$ nas funções $\phi_{\mu \nu l_{1} l_{2} l_{3}}$ do estado fundamental do lítio, para os setores de $\lambda_{9}=2,4,6$ e 8 . Os resultados foram obtidos pelo código "Proj11.map" numa seção de Maple. Assim segue :

Maple V Release 3

$>\operatorname{read}$ 'b1r11.map';

$$
\lambda_{9}:=2
$$

$\rho_{11}\left(\phi_{0,1,0,0,0}\right):=\phi_{0,1,0,0,0}$

$\rho_{11}\left(\phi_{1,0,0,0,0}\right):=0$

$$
\begin{aligned}
& \rho_{11}\left(\phi_{0,0,0,1,1}\right):= \\
& \frac{1}{6} \sqrt{3} \sqrt{2} \phi_{0,0,0,1,1}+\frac{1}{6} \sqrt{3} \sqrt{2} \phi_{0,0,1,0,1}-\frac{1}{3} \sqrt{3} \sqrt{2} \phi_{0,0,1,1,0} \\
& \rho_{11}\left(\phi_{0,0,1,0,1}\right):= \\
& \frac{1}{6} \sqrt{3} \sqrt{2} \phi_{0,0,0,1,1}+\frac{1}{6} \sqrt{3} \sqrt{2} \phi_{0,0,1,0,1}-\frac{1}{3} \sqrt{3} \sqrt{2} \phi_{0,0,1,1,0} \\
& \rho_{11}\left(\phi_{0,0,1,1,0}\right):= \\
& -\frac{1}{6} \sqrt{3} \sqrt{2} \phi_{0,0,0,1,1}-\frac{1}{6} \sqrt{3} \sqrt{2} \phi_{0,0,1,0,1}+\frac{1}{3} \sqrt{3} \sqrt{2} \phi_{0,0,1,1.0} \\
& \text { NumFuncoes }:=5
\end{aligned}
$$

$>$ read'b2r11.map';

$$
\lambda_{9}:=4
$$

$\rho_{11}\left(\phi_{0,2,0,0,0}\right):=\frac{1}{12} \sqrt{11} \sqrt{6} \phi_{0,2,0,0,0}-\frac{1}{12} \sqrt{6} \sqrt{13} \phi_{2,0,0,0,0}$ 
$\rho_{11}\left(\phi_{1,1,0,0,0}\right):=0$

$\rho_{11}\left(\phi_{2,0,0,0,0}\right):=-\frac{1}{12} \sqrt{11} \sqrt{6} \phi_{0,2,0,0,0}+\frac{1}{12} \sqrt{6} \sqrt{13} \phi_{2,0,0,0,0}$

$\rho_{11}\left(\phi_{0,1,0,1,1}\right):=\frac{1}{4} \sqrt{7} \phi_{0,1,0,1,1}-\frac{1}{84} \sqrt{7} \sqrt{3} \sqrt{13} \phi_{1,0,0,1,1}$

$+\frac{1}{4} \sqrt{7} \phi_{0,1,1,0,1}+\frac{1}{84} \sqrt{7} \sqrt{3} \sqrt{13} \phi_{1,0,1,0,1}$

$+\frac{1}{21} \sqrt{7} \sqrt{3} \phi_{0,1,1,1,0}$

$\rho_{11}\left(\phi_{1,0,0,1,1}\right):=-\frac{1}{44} \sqrt{11} \sqrt{13} \phi_{0,1,0,1,1}+\frac{1}{12} \sqrt{3} \sqrt{11} \phi_{1,0,0,1,1}$

$-\frac{1}{44} \sqrt{11} \sqrt{13} \phi_{0,1,1,0,1}-\frac{1}{12} \sqrt{3} \sqrt{11} \phi_{1,0,1,0,1}$

$+\frac{1}{33} \sqrt{3} \sqrt{11} \sqrt{13} \phi_{0,1,1,1,0}$

$\rho_{11}\left(\phi_{0,0,0,2,2}\right):=$

$\frac{1}{6} \sqrt{2} \sqrt{3} \phi_{0,0,0,2,2}+\frac{1}{6} \sqrt{2} \sqrt{3} \phi_{0,0,2,0,2}-\frac{1}{3} \sqrt{2} \sqrt{3} \phi_{0,0,2,2,0}$

$\rho_{11}\left(\phi_{0,1,1,0,1}\right):=\frac{1}{4} \sqrt{7} \phi_{0,1,0,1,1}-\frac{1}{84} \sqrt{7} \sqrt{3} \sqrt{13} \phi_{1,0,0,1,1}$

$+\frac{1}{4} \sqrt{7} \phi_{0,1,1,0,1}+\frac{1}{84} \sqrt{7} \sqrt{3} \sqrt{13} \phi_{1,0,1,0,1}$

$+\frac{1}{21} \sqrt{7} \sqrt{3} \phi_{0,1,1,1,0}$

$\rho_{11}\left(\phi_{1,0,1,0,1}\right):=\frac{1}{44} \sqrt{11} \sqrt{13} \phi_{0,1,0,1,1}-\frac{1}{12} \sqrt{3} \sqrt{11} \phi_{1,0,0,1,1}$

$+\frac{1}{44} \sqrt{11} \sqrt{13} \phi_{0,1,1,0,1}+\frac{1}{12} \sqrt{3} \sqrt{11} \phi_{1,0,1,0,1}$

$-\frac{1}{33} \sqrt{3} \sqrt{11} \sqrt{13} \phi_{0,1,1,1,0}$

$\rho_{11}\left(\phi_{0,1,1,1,0}\right):=\frac{1}{8} \sqrt{2} \phi_{0,1,0,1,1}+\frac{1}{24} \sqrt{3} \sqrt{2} \sqrt{13} \phi_{1,0,0,1,1}$

$+\frac{1}{8} \sqrt{2} \phi_{0,1,1,0.1}-\frac{1}{24} \sqrt{3} \sqrt{2} \sqrt{13} \phi_{1,0,1,0,1}$

$+\frac{1}{3} \sqrt{3} \sqrt{2} \phi_{0,1,1,1,0}$

$\rho_{11}\left(\phi_{1,0,1,1,0}\right):=0$

$\rho_{11}\left(\phi_{0,0,1,1,2}\right):=$

$\frac{1}{3} \sqrt{2} \sqrt{3} \phi_{0,0,1,1,2}-\frac{1}{6} \sqrt{2} \sqrt{3} \phi_{0,0,1,2,1}-\frac{1}{6} \sqrt{2} \sqrt{3} \phi_{0,0,2,1,1}$

$\rho_{11}\left(\phi_{0,0,1,2,1}\right):=$

$-\frac{1}{3} \sqrt{2} \sqrt{3} \phi_{0,0,1,1,2}+\frac{1}{6} \sqrt{2} \sqrt{3} \phi_{0,0,1,2,1}+\frac{1}{6} \sqrt{2} \sqrt{3} \phi_{0,0,2,1,1}$

$\rho_{11}\left(\phi_{0,0,2,0,2}\right):=$

$\frac{1}{6} \sqrt{2} \sqrt{3} \phi_{0,0,0,2,2}+\frac{1}{6} \sqrt{2} \sqrt{3} \phi_{0,0,2,0,2}-\frac{1}{3} \sqrt{2} \sqrt{3} \phi_{0,0,2,2,0}$

$\rho_{11}\left(\phi_{0,0,2,1,1}\right):=$

$-\frac{1}{3} \sqrt{2} \sqrt{3} \phi_{0,0,1,1,2}+\frac{1}{6} \sqrt{2} \sqrt{3} \phi_{0,0,1,2,1}+\frac{1}{6} \sqrt{2} \sqrt{3} \phi_{0,0,2,1,1}$ 
$\rho_{11}\left(\phi_{0,0,2,2,0}\right):=$

$-\frac{1}{6} \sqrt{2} \sqrt{3} \phi_{0,0,0,2,2}-\frac{1}{6} \sqrt{2} \sqrt{3} \phi_{0,0,2,0,2}+\frac{1}{3} \sqrt{2} \sqrt{3} \phi_{0,0,2,2,0}$

NumFuncoes $:=15$

$>$ read'b3r11.map';

$\lambda_{9}:=6$

$$
\begin{aligned}
& \rho_{11}\left(\phi_{0,3,0,0,0}\right):= \frac{1}{4} \sqrt{13} \phi_{0,3,0,0,0}+\frac{1}{4} \sqrt{3} \phi_{2,1,0,0,0} \\
& \rho_{11}\left(\phi_{1,2,0,0,0}\right):=0 \\
& \rho_{11}\left(\phi_{2,1,0,0,0}\right):=\frac{1}{4} \sqrt{13} \phi_{0,3,0,0,0}+\frac{1}{4} \sqrt{3} \phi_{2,1,0,0,0} \\
& \rho_{11}\left(\phi_{3,0,0,0,0)}:=0\right.
\end{aligned}
$$

$\rho_{11}\left(\phi_{0,2,0,1,1}\right):=\frac{5}{24} \sqrt{2} \sqrt{3} \phi_{0,2,0,1,1}+\frac{1}{40} \sqrt{2} \sqrt{7} \sqrt{5} \phi_{1,1,0,1,1}$

$-\frac{1}{20} \sqrt{17} \phi_{2,0,0,1,1}+\frac{5}{24} \sqrt{2} \sqrt{3} \phi_{0,2,1,0,1}$

$-\frac{1}{40} \sqrt{2} \sqrt{7} \sqrt{5} \phi_{1,1,1,0,1}-\frac{1}{20} \sqrt{17} \phi_{2,0,1,0,1}$

$-\frac{1}{30} \sqrt{3} \sqrt{7} \phi_{0,2,1,1,0}-\frac{1}{30} \sqrt{3} \sqrt{5} \sqrt{17} \phi_{2,0,1,1,0}$

$\rho_{11}\left(\phi_{1,1,0,1,1}\right):=\frac{1}{664} \sqrt{2} \sqrt{83} \sqrt{3} \sqrt{7} \sqrt{5} \phi_{0,2,0,1,1}$

$+\frac{1}{24} \sqrt{2} \sqrt{83} \phi_{1,1,0,1,1}-\frac{1}{996} \sqrt{83} \sqrt{5} \sqrt{17} \sqrt{7} \phi_{2,0,0,1,1}$

$+\frac{1}{664} \sqrt{2} \sqrt{83} \sqrt{3} \sqrt{7} \sqrt{5} \phi_{0,2,1,0,1}-\frac{1}{24} \sqrt{2} \sqrt{83} \phi_{1,1,1,0,1}$

$-\frac{1}{996} \sqrt{83} \sqrt{5} \sqrt{17} \sqrt{7} \phi_{2,0,1,0,1}-\frac{5}{498} \sqrt{83} \sqrt{3} \sqrt{5} \phi_{0,2,1,1,0}$

$+\frac{1}{498} \sqrt{83} \sqrt{3} \sqrt{17} \sqrt{7} \phi_{2,0,1,1,0}$

$\rho_{11}\left(\phi_{2,0,0,1,1}\right):=-\frac{1}{328} \sqrt{41} \sqrt{3} \sqrt{2} \sqrt{17} \phi_{0,2,0,1,1}$

$-\frac{1}{984} \sqrt{41} \sqrt{2} \sqrt{17} \sqrt{5} \sqrt{7} \phi_{1,1,0,1,1}+\frac{1}{12} \sqrt{41} \phi_{2,0,0,1,1}$

$-\frac{1}{328} \sqrt{41} \sqrt{3} \sqrt{2} \sqrt{17} \phi_{0,2,1,0,1}$

$+\frac{1}{984} \sqrt{41} \sqrt{2} \sqrt{17} \sqrt{5} \sqrt{7} \phi_{1,1,1,0,1}+\frac{1}{12} \sqrt{41} \phi_{2,0,1,0,1}$

$-\frac{1}{246} \sqrt{41} \sqrt{7} \sqrt{3} \sqrt{17} \phi_{0,2,1,1,0}-\frac{1}{246} \sqrt{41} \sqrt{3} \sqrt{5} \phi_{2,0,1,1,0}$

$\rho_{11}\left(\phi_{0,1,0,2,2}\right):=\frac{3}{10} \sqrt{5} \phi_{0,1,0,2,2}-\frac{1}{90} \sqrt{5} \sqrt{3} \sqrt{17} \phi_{1,0,0,2,2}$

$+\frac{3}{10} \sqrt{5} \phi_{0,1,2,0,2}+\frac{1}{90} \sqrt{5} \sqrt{3} \sqrt{17} \phi_{1,0,2,0,2}+\frac{1}{9} \sqrt{3} \phi_{0,1,2,2,0}$

$\rho_{11}\left(\phi_{1,0,0,2,2}\right):=-\frac{1}{130} \sqrt{5} \sqrt{13} \sqrt{17} \phi_{0,1,0,2,2}$

$$
\begin{aligned}
& +\frac{1}{30} \sqrt{5} \sqrt{13} \sqrt{3} \phi_{1,0,0,2,2}-\frac{1}{130} \sqrt{5} \sqrt{13} \sqrt{17} \phi_{0,1,2,0,2} \\
& -\frac{1}{30} \sqrt{5} \sqrt{13} \sqrt{3} \phi_{1,0,2,0,2}+\frac{1}{39} \sqrt{13} \sqrt{3} \sqrt{17} \phi_{0,1,2,2,0}
\end{aligned}
$$

$\rho_{11}\left(\varphi_{0,0,0,3,3}\right):=$ 
$\frac{1}{6} \sqrt{2} \sqrt{3} \phi_{0,0,0,3,3}+\frac{1}{6} \sqrt{2} \sqrt{3} \phi_{0,0,3,0,3}-\frac{1}{3} \sqrt{2} \sqrt{3} \phi_{0,0,3,3,0}$

$\rho_{11}\left(\phi_{0,2,1,0,1}\right):=\frac{5}{24} \sqrt{2} \sqrt{3} \phi_{0,2,0,1,1}+\frac{1}{40} \sqrt{2} \sqrt{7} \sqrt{5} \phi_{1,1,0,1,1}$

$$
\begin{aligned}
& -\frac{1}{20} \sqrt{17} \phi_{2,0,0,1,1}+\frac{5}{24} \sqrt{2} \sqrt{3} \phi_{0,2,1,0,1} \\
& -\frac{1}{40} \sqrt{2} \sqrt{7} \sqrt{5} \phi_{1,1,1,0,1}-\frac{1}{20} \sqrt{17} \phi_{2,0,1,0,1} \\
& -\frac{1}{30} \sqrt{3} \sqrt{7} \phi_{0,2,1,1,0}-\frac{1}{30} \sqrt{3} \sqrt{5} \sqrt{17} \phi_{2,0,1,1,0}
\end{aligned}
$$

$\rho_{11}\left(\phi_{1,1,1,0,1}\right):=-\frac{1}{664} \sqrt{2} \sqrt{83} \sqrt{3} \sqrt{7} \sqrt{5} \phi_{0,2,0,1,1}$

$$
\begin{aligned}
& -\frac{1}{24} \sqrt{2} \sqrt{83} \phi_{1,1,0,1,1}+\frac{1}{996} \sqrt{83} \sqrt{5} \sqrt{17} \sqrt{7} \phi_{2,0,0,1,1} \\
& -\frac{1}{664} \sqrt{2} \sqrt{83} \sqrt{3} \sqrt{7} \sqrt{5} \phi_{0,2,1,0,1}+\frac{1}{24} \sqrt{2} \sqrt{83} \phi_{1,1,1,0,1} \\
& +\frac{1}{996} \sqrt{83} \sqrt{5} \sqrt{17} \sqrt{7} \phi_{2,0,1,0,1}+\frac{5}{498} \sqrt{83} \sqrt{3} \sqrt{5} \phi_{0,2,1,1,0} \\
& -\frac{1}{498} \sqrt{83} \sqrt{3} \sqrt{17} \sqrt{7} \phi_{2,0,1,1,0}
\end{aligned}
$$

$\rho_{11}\left(\phi_{2,0,1,0,1}\right):=-\frac{1}{328} \sqrt{41} \sqrt{3} \sqrt{2} \sqrt{17} \phi_{0,2,0,1,1}$

$$
\begin{aligned}
& -\frac{1}{984} \sqrt{41} \sqrt{2} \sqrt{17} \sqrt{5} \sqrt{7} \phi_{1,1,0,1,1}+\frac{1}{12} \sqrt{41} \phi_{2,0,0,1,1} \\
& -\frac{1}{328} \sqrt{41} \sqrt{3} \sqrt{2} \sqrt{17} \phi_{0,2,1,0,1} \\
& +\frac{1}{984} \sqrt{41} \sqrt{2} \sqrt{17} \sqrt{5} \sqrt{7} \phi_{1,1,1,0,1}+\frac{1}{12} \sqrt{41} \phi_{2,0,1,0,1} \\
& -\frac{1}{246} \sqrt{41} \sqrt{7} \sqrt{3} \sqrt{17} \phi_{0,2,1,1,0}-\frac{1}{246} \sqrt{41} \sqrt{3} \sqrt{5} \phi_{2,0,1,1,0}
\end{aligned}
$$

$\rho_{11}\left(\phi_{0,2,1,1,0}\right):=-\frac{1}{48} \sqrt{3} \sqrt{7} \phi_{0,2,0,1,1}-\frac{5}{48} \sqrt{5} \phi_{1,1,0,1,1}$

$$
\begin{aligned}
& -\frac{1}{48} \sqrt{2} \sqrt{7} \sqrt{17} \phi_{2,0,0,1,1}-\frac{1}{48} \sqrt{3} \sqrt{7} \phi_{0,2,1,0,1} \\
& +\frac{5}{48} \sqrt{5} \phi_{1,1,1,0,1}-\frac{1}{48} \sqrt{2} \sqrt{7} \sqrt{17} \phi_{2,0,1,0,1} \\
& +\frac{1}{3} \sqrt{2} \sqrt{3} \phi_{0,2,1,1,0}
\end{aligned}
$$

$\rho_{11}\left(\phi_{1,1,1,1,0}\right):=0$

$\rho_{11}\left(\phi_{2,0,1,1,0}\right):=-\frac{1}{48} \sqrt{3} \sqrt{5} \sqrt{17} \phi_{0,2,0,1,1}+\frac{1}{48} \sqrt{17} \sqrt{7} \phi_{1,1,0,1,1}$

$-\frac{1}{48} \sqrt{2} \sqrt{5} \phi_{2,0,0,1,1}-\frac{1}{48} \sqrt{3} \sqrt{5} \sqrt{17} \phi_{0,2,1,0,1}$

$-\frac{1}{48} \sqrt{17} \sqrt{7} \phi_{1,1,1,0,1}-\frac{1}{48} \sqrt{2} \sqrt{5} \phi_{2,0,1,0,1}$

$+\frac{1}{3} \sqrt{2} \sqrt{3} \phi_{2,0,1,1,0}$

$\rho_{11}\left(\phi_{0,1,1,1,2}\right):=\frac{1}{3} \sqrt{2} \sqrt{3} \phi_{0,1,1,1.2}+\frac{1}{12} \sqrt{7} \phi_{0,1,1,2,1}$

$-\frac{1}{12} \sqrt{17} \phi_{1,0,1,2,1}+\frac{1}{12} \sqrt{7} \phi_{0,1,2,1,1}+\frac{1}{12} \sqrt{17} \phi_{1,0,2,1,1}$

$\rho_{11}\left(\phi_{1,0,1,1,2}\right):=0$

$\rho_{11}\left(\phi_{0,1,1,2,1}\right):=\frac{1}{87} \sqrt{29} \sqrt{3} \sqrt{7} \phi_{0,1,1,1,2}+\frac{1}{12} \sqrt{2} \sqrt{29} \phi_{0,1,1,2,1}$

$+\frac{1}{348} \sqrt{2} \sqrt{29} \sqrt{17} \sqrt{7} \phi_{1,0,1,2,1}+\frac{1}{12} \sqrt{2} \sqrt{29} \phi_{0,1,2,1,1}$

$-\frac{1}{348} \sqrt{2} \sqrt{29} \sqrt{17} \sqrt{7} \phi_{1,0,2,1,1}$ 
$\rho_{11}\left(\phi_{1,0,1,2,1}\right):=-\frac{1}{57} \sqrt{19} \sqrt{3} \sqrt{17} \phi_{0,1,1,1,2}$

$+\frac{1}{228} \sqrt{2} \sqrt{19} \sqrt{17} \sqrt{7} \phi_{0,1,1,2,1}+\frac{1}{12} \sqrt{2} \sqrt{19} \phi_{1,0,1,2,1}$

$+\frac{1}{228} \sqrt{2} \sqrt{19} \sqrt{17} \sqrt{7} \phi_{0,1,2,1,1}-\frac{1}{12} \sqrt{2} \sqrt{19} \phi_{1,0,2,1,1}$

$\rho_{11}\left(\phi_{0,0,1,2,3}\right):=\frac{1}{3} \sqrt{3} \phi_{0,0,1,2,3}-\frac{1}{6} \sqrt{3} \phi_{0,0,1,3,2}+\frac{1}{3} \sqrt{3} \phi_{0,0,2,1,3}$ $-\frac{1}{6} \sqrt{3} \phi_{0,0,2,3.1}-\frac{1}{6} \sqrt{3} \phi_{0,0,3,1,2}-\frac{1}{6} \sqrt{3} \phi_{0,0,3,2,1}$.

$\rho_{11}\left(\phi_{0,0,1,3,2}\right):=-\frac{1}{6} \sqrt{3} \phi_{0,0,1,2,3}+\frac{1}{3} \sqrt{3} \phi_{0,0,1,3,2}-\frac{1}{6} \sqrt{3} \phi_{0,0,2,1,3}$ $-\frac{1}{6} \sqrt{3} \phi_{0,0,2,3,1}+\frac{1}{3} \sqrt{3} \phi_{0,0,3,1,2}-\frac{1}{6} \sqrt{3} \phi_{0,0,3,2,1}$

$\rho_{11}\left(\phi_{0,1,2,0,2}\right):=\frac{3}{10} \sqrt{5} \phi_{0,1,0,2.2}-\frac{1}{90} \sqrt{5} \sqrt{3} \sqrt{17} \phi_{1,0,0,2,2}$ $+\frac{3}{10} \sqrt{5} \phi_{0,1,2,0,2}+\frac{1}{90} \sqrt{5} \sqrt{3} \sqrt{17} \phi_{1,0,2,0,2}+\frac{1}{9} \sqrt{3} \phi_{0,1,2,2,0}$

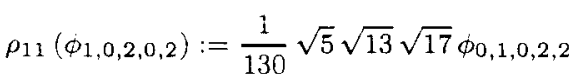

$-\frac{1}{30} \sqrt{5} \sqrt{13} \sqrt{3} \phi_{1,0,0,2,2}+\frac{1}{130} \sqrt{5} \sqrt{13} \sqrt{17} \phi_{0,1,2,0,2}$

$+\frac{1}{30} \sqrt{5} \sqrt{13} \sqrt{3} \phi_{1,0,2,0,2}-\frac{1}{39} \sqrt{13} \sqrt{3} \sqrt{17} \phi_{0,1,2,2,0}$

$\rho_{11}\left(\phi_{0,1,2,1,1}\right):=\frac{1}{87} \sqrt{29} \sqrt{3} \sqrt{7} \phi_{0,1,1,1,2}+\frac{1}{12} \sqrt{2} \sqrt{29} \phi_{0,1,1,2,1}$

$+\frac{1}{348} \sqrt{2} \sqrt{29} \sqrt{17} \sqrt{7} \phi_{1,0,1,2,1}+\frac{1}{12} \sqrt{2} \sqrt{29} \phi_{0,1,2,1,1}$

$-\frac{1}{348} \sqrt{2} \sqrt{29} \sqrt{17} \sqrt{7} \phi_{1,0,2,1,1}$

$\rho_{11}\left(\phi_{1,0,2,1,1}\right):=\frac{1}{57} \sqrt{19} \sqrt{3} \sqrt{17} \phi_{0,1,1,1,2}$

$-\frac{1}{228} \sqrt{2} \sqrt{19} \sqrt{17} \sqrt{7} \phi_{0,1,1,2,1}-\frac{1}{12} \sqrt{2} \sqrt{19} \phi_{1,0,1,2.1}$

$-\frac{1}{228} \sqrt{2} \sqrt{19} \sqrt{17} \sqrt{7} \phi_{0,1,2,1,1}+\frac{1}{12} \sqrt{2} \sqrt{19} \phi_{1,0,2,1,1}$

$\rho_{11}\left(\phi_{0,0,2,1,3}\right):=\frac{1}{3} \sqrt{3} \phi_{0,0,1,2,3}-\frac{1}{6} \sqrt{3} \phi_{0,0,1,3,2}+\frac{1}{3} \sqrt{3} \phi_{0,0,2,1,3}$ $-\frac{1}{6} \sqrt{3} \phi_{0,0,2,3,1}-\frac{1}{6} \sqrt{3} \phi_{0,0,3,1,2}-\frac{1}{6} \sqrt{3} \phi_{0,0,3,2,1}$

$\rho_{11}\left(\phi_{0,1,2,2,0}\right):=\frac{1}{20} \sqrt{2} \sqrt{5} \phi_{0,1,0,2,2}+\frac{1}{60} \sqrt{2} \sqrt{3} \sqrt{5} \sqrt{17} \phi_{1,0,0,2,2}$

$+\frac{1}{20} \sqrt{2} \sqrt{5} \phi_{0,1,2,0,2}-\frac{1}{60} \sqrt{2} \sqrt{3} \sqrt{5} \sqrt{17} \phi_{1,0,2,0,2}$

$+\frac{1}{3} \sqrt{2} \sqrt{3} \phi_{0,1,2,2,0}$

$\rho_{11}\left(\phi_{1,0,2,2,0}\right):=0$

$\rho_{11}\left(\phi_{0,0,2,2,2}\right):=0$

$\rho_{11}\left(\phi_{0,0,2,3,1}\right):=-\frac{1}{6} \sqrt{3} \phi_{0,0,1,2,3}-\frac{1}{6} \sqrt{3} \phi_{0,0,1,3,2}-\frac{1}{6} \sqrt{3} \phi_{0,0,2,1.3}$ $+\frac{1}{3} \sqrt{3} \phi_{0,0,2,3.1}-\frac{1}{6} \sqrt{3} \phi_{0,0,3,1,2}+\frac{1}{3} \sqrt{3} \phi_{0,0,3,2,1}$ 
$\rho_{11}\left(\phi_{0,0,3,0,3}\right):=$ $\frac{1}{6} \sqrt{2} \sqrt{3} \phi_{0,0,0,3,3}+\frac{1}{6} \sqrt{2} \sqrt{3} \phi_{0,0,3,0,3}-\frac{1}{3} \sqrt{2} \sqrt{3} \phi_{0,0,3,3,0}$

$\rho_{11}\left(\phi_{0,0,3,1,2}\right):=-\frac{1}{6} \sqrt{3} \phi_{0,0,1,2,3}+\frac{1}{3} \sqrt{3} \phi_{0,0,1,3,2}-\frac{1}{6} \sqrt{3} \phi_{0,0,2,1,3}$ $-\frac{1}{6} \sqrt{3} \phi_{0,0,2,3,1}+\frac{1}{3} \sqrt{3} \phi_{0,0,3,1,2}-\frac{1}{6} \sqrt{3} \phi_{0,0,3,2,1}$

$\rho_{11}\left(\phi_{0,0,3,2,1}\right):=-\frac{1}{6} \sqrt{3} \phi_{0,0,1,2,3}-\frac{1}{6} \sqrt{3} \phi_{0,0,1,3,2}-\frac{1}{6} \sqrt{3} \phi_{0,0,2,1,3}$ $+\frac{1}{3} \sqrt{3} \phi_{0,0,2,3,1}-\frac{1}{6} \sqrt{3} \phi_{0,0,3,1,2}+\frac{1}{3} \sqrt{3} \phi_{0,0,3,2,1}$

$\rho_{11}\left(\phi_{0,0,3,3,0}\right):=$

$-\frac{1}{6} \sqrt{2} \sqrt{3} \phi_{0,0,0,3,3}-\frac{1}{6} \sqrt{2} \sqrt{3} \phi_{0,0,3,0,3}+\frac{1}{3} \sqrt{2} \sqrt{3} \phi_{0,0,3,3,0}$

NumFuncoes $:=35$

read 'b4r11.map';

$$
\lambda_{9}:=8
$$

$\rho_{11}\left(\phi_{0,4,0,0,0}\right):=\frac{1}{24} \sqrt{3} \sqrt{107} \phi_{0,4,0,0,0}$

$-\frac{3}{1712} \sqrt{3} \sqrt{107} \sqrt{17} \sqrt{10} \phi_{2,2,0,0,0}$

$-\frac{1}{5136} \sqrt{3} \sqrt{107} \sqrt{17} \sqrt{133} \sqrt{10} \phi_{4,0,0,0,0}$

$\rho_{11}\left(\phi_{1,3,0,0,0}\right):=0$

$\rho_{11}\left(\phi_{2,2,0,0,0}\right):=-\frac{3}{808} \sqrt{101} \sqrt{17} \sqrt{5} \phi_{0,4,0,0,0}$

$+\frac{1}{16} \sqrt{2} \sqrt{101} \phi_{2,2,0,0,0}-\frac{3}{1616} \sqrt{2} \sqrt{101} \sqrt{133} \phi_{4,0,0,0,0}$

$\rho_{11}\left(\phi_{3,1,0,0,0}\right):=0$

$\rho_{11}\left(\phi_{4,0,0,0,0}\right):=-\frac{1}{6024} \sqrt{3} \sqrt{251} \sqrt{133} \sqrt{17} \sqrt{5} \phi_{0,4,0,0,0}$

$-\frac{3}{4016} \sqrt{3} \sqrt{2} \sqrt{251} \sqrt{133} \phi_{2,2,0,0,0}+\frac{1}{48} \sqrt{3} \sqrt{2} \sqrt{251} \phi_{4,0,0,0,0}$

$$
\begin{aligned}
\rho_{11}( & \left.\phi_{0,3,0,1,1}\right):=\frac{1}{48} \sqrt{2} \sqrt{3} \sqrt{149} \phi_{0,3,0,1,1} \\
& -\frac{1}{2384} \sqrt{2} \sqrt{3} \sqrt{149} \sqrt{17} \sqrt{7} \phi_{1,2,0,1,1} \\
& +\frac{1}{2384} \sqrt{3} \sqrt{149} \sqrt{17} \sqrt{19} \phi_{2,1,0,1,1} \\
& -\frac{1}{7152} \sqrt{3} \sqrt{149} \sqrt{17} \sqrt{7} \sqrt{19} \phi_{3,0,0,1,1} \\
& +\frac{1}{48} \sqrt{2} \sqrt{3} \sqrt{149} \phi_{0,3,1,0,1} \\
& +\frac{1}{2384} \sqrt{2} \sqrt{3} \sqrt{149} \sqrt{17} \sqrt{7} \phi_{1,2,1,0,1} \\
& +\frac{1}{2384} \sqrt{3} \sqrt{149} \sqrt{17} \sqrt{19} \phi_{2,1,1,0,1} \\
& +\frac{1}{7152} \sqrt{3} \sqrt{149} \sqrt{17} \sqrt{7} \sqrt{19} \phi_{3,0,1,0,1} \\
& +\frac{1}{596} \sqrt{2} \sqrt{149} \sqrt{7} \phi_{0,3,1,1,0}
\end{aligned}
$$


$+\frac{1}{1788} \sqrt{2} \sqrt{3} \sqrt{149} \sqrt{17} \sqrt{19} \phi_{2,1,1,1,0}$

$\rho_{11}\left(\phi_{1,2,0,1,1}\right):=-\frac{3}{7568} \sqrt{2} \sqrt{11} \sqrt{43} \sqrt{17} \sqrt{7} \phi_{0,3,0,1,1}$

$+\frac{1}{48} \sqrt{2} \sqrt{11} \sqrt{43} \phi_{1,2,0,1,1}+\frac{1}{22704} \sqrt{11} \sqrt{43} \sqrt{19} \sqrt{7} \phi_{2,1,0,1,1}$

$-\frac{15}{7568} \sqrt{11} \sqrt{43} \sqrt{19} \phi_{3,0,0,1,1}$

$-\frac{3}{7568} \sqrt{2} \sqrt{11} \sqrt{43} \sqrt{17} \sqrt{7} \phi_{0,3,1,0,1}$

$-\frac{1}{48} \sqrt{2} \sqrt{11} \sqrt{43} \phi_{1,2,1,0,1}+\frac{1}{22704} \sqrt{11} \sqrt{43} \sqrt{19} \sqrt{7} \phi_{2,1,1,0,1}$

$+\frac{15}{7568} \sqrt{11} \sqrt{43} \sqrt{19} \phi_{3,0,1,0,1}$

$+\frac{5}{5676} \sqrt{2} \sqrt{11} \sqrt{43} \sqrt{3} \sqrt{17} \phi_{0,3,1,1,0}$

$+\frac{1}{1892} \sqrt{2} \sqrt{11} \sqrt{43} \sqrt{19} \sqrt{7} \phi_{2,1,1,1,0}$

$\rho_{11}\left(\phi_{2,1,0,1,1}\right):=\frac{3}{8144} \sqrt{509} \sqrt{17} \sqrt{19} \sqrt{2} \phi_{0,3,0,1,1}$

$+\frac{1}{24432} \sqrt{509} \sqrt{19} \sqrt{2} \sqrt{7} \phi_{1,2,0,1,1}+\frac{1}{48} \sqrt{509} \phi_{2,1,0,1,1}$

$-\frac{21}{8144} \sqrt{509} \sqrt{7} \phi_{3,0,0,1,1}+\frac{3}{8144} \sqrt{509} \sqrt{17} \sqrt{19} \sqrt{2} \phi_{0,3,1,0,1}$

$-\frac{1}{24432} \sqrt{509} \sqrt{19} \sqrt{2} \sqrt{7} \phi_{1,2,1,0,1}+\frac{1}{48} \sqrt{509} \phi_{2,1,1,0,1}$

$+\frac{21}{8144} \sqrt{509} \sqrt{7} \phi_{3,0,1,0,1}$

$+\frac{1}{6108} \sqrt{509} \sqrt{2} \sqrt{7} \sqrt{3} \sqrt{17} \sqrt{19} \phi_{0,3,1,1,0}$

$-\frac{31}{2036} \sqrt{509} \sqrt{2} \phi_{2,1,1,1,0}$

$\rho_{11}\left(\phi_{3,0,0,1,1}\right):=-\frac{1}{11568} \sqrt{3} \sqrt{241} \sqrt{17} \sqrt{7} \sqrt{19} \sqrt{2} \phi_{0,3,0,1,1}$

$-\frac{5}{3856} \sqrt{3} \sqrt{241} \sqrt{19} \sqrt{2} \phi_{1,2,0,1,1}$

$-\frac{7}{3856} \sqrt{3} \sqrt{241} \sqrt{7} \phi_{2,1,0,1,1}+\frac{1}{48} \sqrt{3} \sqrt{241} \phi_{3,0,0,1,1}$

$-\frac{1}{11568} \sqrt{3} \sqrt{241} \sqrt{17} \sqrt{7} \sqrt{19} \sqrt{2} \phi_{0,3,1,0,1}$

$+\frac{5}{3856} \sqrt{3} \sqrt{241} \sqrt{19} \sqrt{2} \phi_{1,2,1,0,1}$

$-\frac{7}{3856} \sqrt{3} \sqrt{241} \sqrt{7} \phi_{2,1,1,0,1}-\frac{1}{48} \sqrt{3} \sqrt{241} \phi_{3,0,1,0,1}$

$+\frac{1}{964} \sqrt{241} \sqrt{2} \sqrt{17} \sqrt{19} \phi_{0,3,1,1,0}$

$+\frac{5}{2892} \sqrt{3} \sqrt{241} \sqrt{2} \sqrt{7} \phi_{2,1,1,1,0}$

$\rho_{11}\left(\phi_{0,2,0,2,2}\right):=\frac{1}{60} \sqrt{3} \sqrt{5} \sqrt{59} \phi_{0,2,0,2,2}$

$+\frac{1}{590} \sqrt{3} \sqrt{5} \sqrt{59} \sqrt{19} \phi_{1,1,0,2,2}$

$-\frac{1}{708} \sqrt{3} \sqrt{5} \sqrt{59} \sqrt{19} \phi_{2,0,0,2,2}+\frac{1}{60} \sqrt{3} \sqrt{5} \sqrt{59} \phi_{0,2,2,0,2}$

$-\frac{1}{590} \sqrt{3} \sqrt{5} \sqrt{59} \sqrt{19} \phi_{1,1,2,0,2}$

$-\frac{1}{708} \sqrt{3} \sqrt{5} \sqrt{59} \sqrt{19} \phi_{2,0,2,0,2}-\frac{1}{236} \sqrt{3} \sqrt{5} \sqrt{59} \phi_{0,2,2,2,0}$

$-\frac{7}{708} \sqrt{3} \sqrt{59} \sqrt{19} \phi_{2,0,2,2,0}$

$\rho_{11}\left(\phi_{1,1,0,2,2)}\right)=\frac{1}{20} \sqrt{3} \sqrt{5} \varphi_{0,2,0,2.2}-\frac{1}{30} \sqrt{3} \sqrt{5} \sqrt{19} \varphi_{1,1,0.2 .2}$ 
$-\frac{1}{76} \sqrt{3} \sqrt{5} \sqrt{19} \phi_{2,0,0,2,2}+\frac{1}{20} \sqrt{3} \sqrt{5} \phi_{0,2,2,0,2}$

$-\frac{1}{30} \sqrt{3} \sqrt{5} \sqrt{19} \phi_{1,1,2,0,2}-\frac{1}{76} \sqrt{3} \sqrt{5} \sqrt{19} \phi_{2,0,2,0,2}$

$-\frac{1}{12} \sqrt{3} \sqrt{5} \phi_{0,2,2,2,0}+\frac{3}{76} \sqrt{3} \sqrt{19} \phi_{2,0,2,2,0}$

$\rho_{11}\left(\phi_{2,0,0,2,2}\right):=-\frac{1}{156} \sqrt{3} \sqrt{13} \sqrt{19} \phi_{0,2,0,2,2}-\frac{1}{26} \sqrt{3} \sqrt{13} \phi_{1,1,0,2,2}$

$+\frac{1}{12} \sqrt{3} \sqrt{13} \phi_{2,0,0,2,2}-\frac{1}{156} \sqrt{3} \sqrt{13} \sqrt{19} \phi_{0,2,2,0.2}$

$+\frac{1}{26} \sqrt{3} \sqrt{13} \phi_{1,1,2,0,2}+\frac{1}{12} \sqrt{3} \sqrt{13} \phi_{2,0,2,0,2}$

$-\frac{1}{52} \sqrt{3} \sqrt{13} \sqrt{19} \phi_{0,2,2,2,0}-\frac{1}{156} \sqrt{3} \sqrt{13} \sqrt{5} \phi_{2,0,2,2,0}$

$\rho_{11}\left(\phi_{0,1,0,3,3}\right):=\frac{1}{12} \sqrt{2} \sqrt{11} \sqrt{3} \phi_{0,1,0,3,3}$

$-\frac{1}{132} \sqrt{2} \sqrt{11} \sqrt{3} \sqrt{7} \phi_{1,0,0,3,3}+\frac{1}{12} \sqrt{2} \sqrt{11} \sqrt{3} \phi_{0,1,3,0,3}$

$+\frac{1}{132} \sqrt{2} \sqrt{11} \sqrt{3} \sqrt{7} \phi_{1,0,3,0,3}+\frac{1}{33} \sqrt{11} \sqrt{3} \phi_{0,1,3,3,0}$

$\rho_{11}\left(\phi_{1,0,0,3,3}\right):=-\frac{1}{60} \sqrt{2} \sqrt{5} \sqrt{3} \sqrt{7} \phi_{0,1,0,3,3}$

$+\frac{1}{12} \sqrt{2} \sqrt{5} \sqrt{3} \phi_{1,0,0,3,3}-\frac{1}{60} \sqrt{2} \sqrt{5} \sqrt{3} \sqrt{7} \phi_{0,1,3,0,3}$

$-\frac{1}{12} \sqrt{2} \sqrt{5} \sqrt{3} \phi_{1,0,3,0,3}+\frac{1}{15} \sqrt{5} \sqrt{3} \sqrt{7} \phi_{0,1,3,3,0}$

$\rho_{11}\left(\phi_{0,0,0,4,4}\right):=$

$\frac{1}{6} \sqrt{2} \sqrt{3} \phi_{0,0,0,4,4}+\frac{1}{6} \sqrt{2} \sqrt{3} \phi_{0,0,4,0,4}-\frac{1}{3} \sqrt{2} \sqrt{3} \phi_{0,0,4,4,0}$

$\rho_{11}\left(\phi_{0,3,1,0,1}\right):=\frac{1}{48} \sqrt{2} \sqrt{3} \sqrt{149} \phi_{0,3,0,1,1}$

$-\frac{1}{2384} \sqrt{2} \sqrt{3} \sqrt{149} \sqrt{17} \sqrt{7} \phi_{1,2,0,1,1}$

$+\frac{1}{2384} \sqrt{3} \sqrt{149} \sqrt{17} \sqrt{19} \phi_{2,1,0,1,1}$

$-\frac{1}{7152} \sqrt{3} \sqrt{149} \sqrt{17} \sqrt{7} \sqrt{19} \phi_{3,0,0,1,1}$

$+\frac{1}{48} \sqrt{2} \sqrt{3} \sqrt{149} \phi_{0,3,1,0,1}$

$+\frac{1}{2384} \sqrt{2} \sqrt{3} \sqrt{149} \sqrt{17} \sqrt{7} \phi_{1,2,1,0,1}$

$+\frac{1}{2384} \sqrt{3} \sqrt{149} \sqrt{17} \sqrt{19} \phi_{2,1,1,0,1}$

$+\frac{1}{7152} \sqrt{3} \sqrt{149} \sqrt{17} \sqrt{7} \sqrt{19} \phi_{3,0,1,0,1}$

$+\frac{1}{596} \sqrt{2} \sqrt{149} \sqrt{7} \phi_{0,3,1,1,0}$

$+\frac{1}{1788} \sqrt{2} \sqrt{3} \sqrt{149} \sqrt{17} \sqrt{19} \phi_{2,1,1,1,0}$

$\rho_{11}\left(\phi_{1,2,1,0,1}\right):=\frac{3}{7568} \sqrt{2} \sqrt{11} \sqrt{43} \sqrt{17} \sqrt{7} \phi_{0,3,0,1,1}$

$-\frac{1}{48} \sqrt{2} \sqrt{11} \sqrt{43} \phi_{1,2,0,1,1}-\frac{1}{22704} \sqrt{11} \sqrt{43} \sqrt{19} \sqrt{7} \phi_{2,1,0,1,1}$

$+\frac{15}{7568} \sqrt{11} \sqrt{43} \sqrt{19} \phi_{3,0,0,1,1}$

$+\frac{3}{7568} \sqrt{2} \sqrt{11} \sqrt{43} \sqrt{17} \sqrt{7} \phi_{0,3,1,0,1}$

$+\frac{1}{48} \sqrt{2} \sqrt{11} \sqrt{43} \phi_{1,2,1,0,1}-\frac{1}{22704} \sqrt{11} \sqrt{43} \sqrt{19} \sqrt{7} \phi_{2,1,1,0.1}$

$-\frac{15}{7565} \sqrt{11} \sqrt{43} \sqrt{19} \phi_{3,0,1,0.1}$ 
$-\frac{5}{5676} \sqrt{2} \sqrt{11} \sqrt{43} \sqrt{3} \sqrt{17} \phi_{0,3,1,1,0}$

$-\frac{1}{1892} \sqrt{2} \sqrt{11} \sqrt{43} \sqrt{19} \sqrt{7} \phi_{2,1,1,1,0}$

$$
\begin{aligned}
\rho_{11}( & \left.\phi_{2,1,1,0,1}\right):=\frac{3}{8144} \sqrt{509} \sqrt{17} \sqrt{19} \sqrt{2} \phi_{0,3,0,1,1} \\
& +\frac{1}{24432} \sqrt{509} \sqrt{19} \sqrt{2} \sqrt{7} \phi_{1,2,0,1,1}+\frac{1}{48} \sqrt{509} \phi_{2,1,0,1,1} \\
& -\frac{21}{8144} \sqrt{509} \sqrt{7} \phi_{3,0,0,1,1}+\frac{3}{8144} \sqrt{509} \sqrt{17} \sqrt{19} \sqrt{2} \phi_{0,3,1,0,1} \\
& -\frac{1}{24432} \sqrt{509} \sqrt{19} \sqrt{2} \sqrt{7} \phi_{1,2,1,0,1}+\frac{1}{48} \sqrt{509} \phi_{2,1,1,0,1} \\
& +\frac{21}{8144} \sqrt{509} \sqrt{7} \phi_{3,0,1,0,1} \\
& +\frac{1}{6108} \sqrt{509} \sqrt{2} \sqrt{7} \sqrt{3} \sqrt{17} \sqrt{19} \phi_{0,3,1,1,0} \\
& -\frac{31}{2036} \sqrt{509} \sqrt{2} \phi_{2,1,1,1,0}
\end{aligned}
$$

$\rho_{11}\left(\phi_{3,0.1,0,1}\right):=\frac{1}{11568} \sqrt{3} \sqrt{241} \sqrt{17} \sqrt{7} \sqrt{19} \sqrt{2} \phi_{0,3,0,1,1}$

$$
\begin{aligned}
& +\frac{5}{3856} \sqrt{3} \sqrt{241} \sqrt{19} \sqrt{2} \phi_{1,2,0,1,1} \\
& +\frac{7}{3856} \sqrt{3} \sqrt{241} \sqrt{7} \phi_{2,1,0,1,1}-\frac{1}{48} \sqrt{3} \sqrt{241} \phi_{3,0,0,1,1} \\
& +\frac{1}{11568} \sqrt{3} \sqrt{241} \sqrt{17} \sqrt{7} \sqrt{19} \sqrt{2} \phi_{0,3,1,0,1} \\
& -\frac{5}{3856} \sqrt{3} \sqrt{241} \sqrt{19} \sqrt{2} \phi_{1,2,1,0,1} \\
& +\frac{7}{3856} \sqrt{3} \sqrt{241} \sqrt{7} \phi_{2,1,1,0,1}+\frac{1}{48} \sqrt{3} \sqrt{241} \phi_{3,0,1,0,1} \\
& -\frac{1}{964} \sqrt{241} \sqrt{2} \sqrt{17} \sqrt{19} \phi_{0,3,1,1,0} \\
& -\frac{5}{2892} \sqrt{3} \sqrt{241} \sqrt{2} \sqrt{7} \phi_{2,1,1,1,0}
\end{aligned}
$$

$\rho_{11}\left(\phi_{0,3,1,1,0}\right):=\frac{1}{64} \sqrt{2} \sqrt{7} \phi_{0,3,0,1,1}+\frac{5}{192} \sqrt{2} \sqrt{17} \phi_{1,2,0,1,1}$

$$
\begin{aligned}
& +\frac{1}{192} \sqrt{7} \sqrt{17} \sqrt{19} \phi_{2,1,0,1,1}+\frac{1}{64} \sqrt{17} \sqrt{19} \phi_{3,0,0,1,1} \\
& +\frac{1}{64} \sqrt{2} \sqrt{7} \phi_{0,3,1,0,1}-\frac{5}{192} \sqrt{2} \sqrt{17} \phi_{1,2,1,0,1} \\
& +\frac{1}{192} \sqrt{7} \sqrt{17} \sqrt{19} \phi_{2,1,1,0,1}-\frac{1}{64} \sqrt{17} \sqrt{19} \phi_{3,0,1,0,1} \\
& +\frac{1}{3} \sqrt{2} \sqrt{3} \phi_{0,3,1,1,0} \\
& \rho_{11}\left(\phi_{1,2,1,1,0}\right):=0 \\
& \rho_{11}\left(\phi_{2,1,1,1,0}\right):=\frac{1}{192} \sqrt{2} \sqrt{3} \sqrt{17} \sqrt{19} \phi_{0,3,0,1,1} \\
& +\frac{1}{192} \sqrt{2} \sqrt{3} \sqrt{19} \sqrt{7} \phi_{1,2,0,1,1}-\frac{31}{192} \sqrt{3} \phi_{2,1,0,1,1} \\
& +\frac{5}{192} \sqrt{3} \sqrt{7} \phi_{3,0,0,1,1}+\frac{1}{192} \sqrt{2} \sqrt{3} \sqrt{17} \sqrt{19} \phi_{0,3,1,0,1} \\
& -\frac{1}{192} \sqrt{2} \sqrt{3} \sqrt{19} \sqrt{7} \phi_{1,2,1,0,1}-\frac{31}{192} \sqrt{3} \phi_{2,1,1,0,1} \\
& -\frac{5}{192} \sqrt{3} \sqrt{7} \phi_{3,0,1,0,1}+\frac{1}{3} \sqrt{2} \sqrt{3} \phi_{2,1,1,1,0} \\
& \rho_{11}\left(\phi_{3,0,1,1,0}\right):=0 \\
& \rho_{11}\left(\phi_{0,2,1,1,2}\right):=\frac{1}{3} \sqrt{3} \sqrt{2} \phi_{0,2,1,1,2}-\frac{1}{48} \sqrt{3} \sqrt{2} \sqrt{7} \phi_{0,2,1,2,1} \\
& +\frac{1}{96} \sqrt{3} \sqrt{2} \sqrt{5} \sqrt{19} \phi_{1,1,1,2,1}-\frac{1}{96} \sqrt{3} \sqrt{2} \sqrt{7} \sqrt{19} \phi_{\varphi_{2,0.1,2 .}}
\end{aligned}
$$


$-\frac{1}{48} \sqrt{3} \sqrt{2} \sqrt{7} \phi_{0,2,2,1,1}-\frac{1}{96} \sqrt{3} \sqrt{2} \sqrt{5} \sqrt{19} \phi_{1,1,2,1,1}$

$-\frac{1}{96} \sqrt{3} \sqrt{2} \sqrt{7} \sqrt{19} \phi_{2,0,2,1,1}$

$$
\rho_{11}\left(\phi_{1,1,1,1,2}\right):=0
$$

$\rho_{11}\left(\phi_{2,0,1,1,2}\right):=\frac{1}{3} \sqrt{3} \sqrt{2} \phi_{2,0,1,1,2}-\frac{1}{48} \sqrt{2} \sqrt{5} \sqrt{19} \phi_{0,2,1,2,1}$

$-\frac{7}{96} \sqrt{2} \sqrt{7} \phi_{1,1,1,2,1}-\frac{1}{32} \sqrt{2} \sqrt{5} \phi_{2,0,1,2,1}$

$-\frac{1}{48} \sqrt{2} \sqrt{5} \sqrt{19} \phi_{0,2,2,1,1}+\frac{7}{96} \sqrt{2} \sqrt{7} \phi_{1,1,2,1,1}$

$-\frac{1}{32} \sqrt{2} \sqrt{5} \phi_{2,0,2,1,1}$

$\rho_{11}\left(\phi_{0,2,1,2,1}\right):=-\frac{1}{86} \sqrt{43} \sqrt{7} \phi_{0,2,1,1,2}$

$-\frac{1}{258} \sqrt{43} \sqrt{3} \sqrt{5} \sqrt{19} \phi_{2,0,1,1,2}+\frac{1}{12} \sqrt{43} \phi_{0,2,1,2,1}$

$-\frac{1}{1032} \sqrt{43} \sqrt{5} \sqrt{19} \sqrt{7} \phi_{1,1,1,2,1}-\frac{3}{344} \sqrt{43} \sqrt{19} \phi_{2,0,1,2,1}$

$+\frac{1}{12} \sqrt{43} \phi_{0,2,2,1,1}+\frac{1}{1032} \sqrt{43} \sqrt{5} \sqrt{19} \sqrt{7} \phi_{1,1,2,1,1}$

$-\frac{3}{344} \sqrt{43} \sqrt{19} \phi_{2,0,2,1,1}$

$\rho_{11}\left(\phi_{1,1,1,2,1}\right):=\frac{1}{262} \sqrt{131} \sqrt{5} \sqrt{19} \phi_{0,2,1,1,2}$

$-\frac{7}{786} \sqrt{131} \sqrt{3} \sqrt{7} \phi_{2,0,1,1,2}-\frac{1}{1572} \sqrt{131} \sqrt{5} \sqrt{19} \sqrt{7} \phi_{0,2,1,2,1}$

$+\frac{1}{24} \sqrt{131} \phi_{1,1,1,2,1}+\frac{3}{1048} \sqrt{131} \sqrt{5} \sqrt{7} \phi_{2,0,1,2,1}$

$-\frac{1}{1572} \sqrt{131} \sqrt{5} \sqrt{19} \sqrt{7} \phi_{0,2,2,1,1}-\frac{1}{24} \sqrt{131} \phi_{1,1,2,1,1}$

$+\frac{3}{1048} \sqrt{131} \sqrt{5} \sqrt{7} \phi_{2,0,2,1,1}$

$\rho_{11}\left(\phi_{2,0,1,2,1}\right):=-\frac{1}{354} \sqrt{3} \sqrt{59} \sqrt{19} \sqrt{7} \phi_{0,2,1,1,2}$

$-\frac{1}{118} \sqrt{59} \sqrt{5} \phi_{2,0,1,1,2}-\frac{1}{236} \sqrt{3} \sqrt{59} \sqrt{19} \phi_{0,2,1,2,1}$

$+\frac{1}{472} \sqrt{3} \sqrt{59} \sqrt{5} \sqrt{7} \phi_{1,1,1,2,1}+\frac{1}{24} \sqrt{3} \sqrt{59} \phi_{2,0,1,2,1}$

$-\frac{1}{236} \sqrt{3} \sqrt{59} \sqrt{19} \phi_{0,2,2,1,1}-\frac{1}{472} \sqrt{3} \sqrt{59} \sqrt{5} \sqrt{7} \phi_{1,1,2,1,1}$

$+\frac{1}{24} \sqrt{3} \sqrt{59} \phi_{2,0,2,1,1}$

$\rho_{11}\left(\phi_{0,1,1,2,3}\right):=\frac{1}{3} \sqrt{3} \phi_{0,1,1,2,3}+\frac{1}{8} \sqrt{2} \phi_{0,1,1,3,2}$

$-\frac{1}{24} \sqrt{3} \sqrt{5} \sqrt{2} \phi_{1,0,1,3,2}+\frac{1}{3} \sqrt{3} \phi_{0,1,2,1,3}+\frac{1}{16} \sqrt{5} \phi_{0,1,2,3,1}$

$-\frac{7}{48} \sqrt{3} \phi_{1,0,2,3,1}+\frac{1}{8} \sqrt{2} \phi_{0,1,3,1,2}+\frac{1}{24} \sqrt{3} \sqrt{5} \sqrt{2} \phi_{1,0,3,1,2}$

$+\frac{1}{16} \sqrt{5} \phi_{0,1,3,2,1}+\frac{7}{48} \sqrt{3} \phi_{1,0,3,2,1}$

$\rho_{11}\left(\phi_{1,0,1,2,3}\right):=\frac{1}{3} \sqrt{3} \phi_{1,0,1,2,3}-\frac{1}{24} \sqrt{3} \sqrt{2} \sqrt{5} \phi_{0,1,1,3,2}$

$$
\begin{aligned}
& -\frac{1}{8} \sqrt{2} \phi_{1,0,1,3,2}-\frac{1}{3} \sqrt{3} \phi_{1,0.2 .1 .3}+\frac{7}{48} \sqrt{3} \phi_{0,1,2,3.1} \\
& +\frac{1}{16} \sqrt{5} \phi_{1,0,2,3,1}-\frac{1}{24} \sqrt{3} \sqrt{2} \sqrt{5} \phi_{0,1,3,1,2}+\frac{1}{8} \sqrt{2} \phi_{1,0,3,1,2} \\
& +\frac{7}{48} \sqrt{3} \phi_{0,1,3.2,1}-\frac{1}{16} \sqrt{5} \phi_{1,0,3,2.1}
\end{aligned}
$$


$\rho_{11}\left(\phi_{0,1,1,3,2}\right):=\frac{1}{8} \sqrt{2} \phi_{0,1,1,2,3}-\frac{1}{24} \sqrt{3} \sqrt{2} \sqrt{5} \phi_{1,0,1,2,3}$

$+\frac{1}{3} \sqrt{3} \phi_{0,1,1,3,2}+\frac{1}{8} \sqrt{2} \phi_{0,1,2,1,3}+\frac{1}{24} \sqrt{3} \sqrt{2} \sqrt{5} \phi_{1,0,2,1,3}$

$+\frac{1}{48} \sqrt{3} \sqrt{5} \sqrt{2} \phi_{0,1,2,3,1}+\frac{3}{16} \sqrt{2} \phi_{1,0,2,3,1}+\frac{1}{3} \sqrt{3} \phi_{0,1,3,1,2}$

$+\frac{1}{48} \sqrt{3} \sqrt{5} \sqrt{2} \phi_{0,1,3,2,1}-\frac{3}{16} \sqrt{2} \phi_{1,0,3,2,1}$

$\rho_{11}\left(\phi_{1,0,1,3,2}\right):=-\frac{1}{24} \sqrt{3} \sqrt{5} \sqrt{2} \phi_{0,1,1,2,3}-\frac{1}{8} \sqrt{2} \phi_{1,0,1,2,3}$

$+\frac{1}{3} \sqrt{3} \phi_{1,0,1,3,2}-\frac{1}{24} \sqrt{3} \sqrt{5} \sqrt{2} \phi_{0,1,2,1,3}+\frac{1}{8} \sqrt{2} \phi_{1,0,2,1,3}$

$+\frac{3}{16} \sqrt{2} \phi_{0,1,2,3,1}-\frac{1}{48} \sqrt{3} \sqrt{5} \sqrt{2} \phi_{1,0,2,3,1}-\frac{1}{3} \sqrt{3} \phi_{1,0,3,1,2}$

$+\frac{3}{16} \sqrt{2} \phi_{0,1,3,2,1}+\frac{1}{48} \sqrt{3} \sqrt{5} \sqrt{2} \phi_{1,0,3,2,1}$

$\rho_{11}\left(\phi_{0,0,1,3,4}\right):=\frac{1}{3} \sqrt{3} \phi_{0,0,1,3,4}-\frac{1}{6} \sqrt{3} \phi_{0,0,1,4,3}+\frac{1}{3} \sqrt{3} \phi_{0,0,3,1,4}$ $-\frac{1}{6} \sqrt{3} \phi_{0,0,3,4,1}-\frac{1}{6} \sqrt{3} \phi_{0,0,4,1,3}-\frac{1}{6} \sqrt{3} \phi_{0,0,4,3,1}$

$\rho_{11}\left(\phi_{0,0,1,4,3}\right):=-\frac{1}{6} \sqrt{3} \phi_{0,0,1,3,4}+\frac{1}{3} \sqrt{3} \phi_{0,0,1,4,3}-\frac{1}{6} \sqrt{3} \phi_{0,0,3,1,4}$ $-\frac{1}{6} \sqrt{3} \phi_{0,0,3,4,1}+\frac{1}{3} \sqrt{3} \phi_{0,0,4,1,3}-\frac{1}{6} \sqrt{3} \phi_{0,0,4,3,1}$

$\rho_{11}\left(\phi_{0,2,2,0,2}\right):=\frac{1}{60} \sqrt{3} \sqrt{5} \sqrt{59} \phi_{0,2,0,2,2}$

$+\frac{1}{590} \sqrt{3} \sqrt{5} \sqrt{59} \sqrt{19} \phi_{1,1,0,2,2}$

$-\frac{1}{708} \sqrt{3} \sqrt{5} \sqrt{59} \sqrt{19} \phi_{2,0,0,2,2}+\frac{1}{60} \sqrt{3} \sqrt{5} \sqrt{59} \phi_{0,2,2,0,2}$

$-\frac{1}{590} \sqrt{3} \sqrt{5} \sqrt{59} \sqrt{19} \phi_{1,1,2,0,2}$

$-\frac{1}{708} \sqrt{3} \sqrt{5} \sqrt{59} \sqrt{19} \phi_{2,0,2,0,2}-\frac{1}{236} \sqrt{3} \sqrt{5} \sqrt{59} \phi_{0,2,2,2,0}$

$-\frac{7}{708} \sqrt{3} \sqrt{59} \sqrt{19} \phi_{2,0,2,2,0}$

$\rho_{11}\left(\phi_{1,1,2,0,2}\right):=-\frac{1}{20} \sqrt{3} \sqrt{5} \phi_{0,2,0,2,2}-\frac{1}{30} \sqrt{3} \sqrt{5} \sqrt{19} \phi_{1,1,0,2,2}$

$+\frac{1}{76} \sqrt{3} \sqrt{5} \sqrt{19} \phi_{2,0,0,2,2}-\frac{1}{20} \sqrt{3} \sqrt{5} \phi_{0,2,2,0,2}$

$+\frac{1}{30} \sqrt{3} \sqrt{5} \sqrt{19} \phi_{1,1,2,0,2}+\frac{1}{76} \sqrt{3} \sqrt{5} \sqrt{19} \phi_{2,0,2,0,2}$

$+\frac{1}{12} \sqrt{3} \sqrt{5} \phi_{0,2,2,2,0}-\frac{3}{76} \sqrt{3} \sqrt{19} \phi_{2,0,2,2,0}$

$\rho_{11}\left(\phi_{2,0,2,0,2}\right):=-\frac{1}{156} \sqrt{3} \sqrt{13} \sqrt{19} \phi_{0,2,0,2,2}-\frac{1}{26} \sqrt{3} \sqrt{13} \phi_{1,1,0,2,2}$

$+\frac{1}{12} \sqrt{3} \sqrt{13} \phi_{2,0,0,2,2}-\frac{1}{156} \sqrt{3} \sqrt{13} \sqrt{19} \phi_{0,2,2,0,2}$

$+\frac{1}{26} \sqrt{3} \sqrt{13} \phi_{1,1,2,0,2}+\frac{1}{12} \sqrt{3} \sqrt{13} \phi_{2,0,2,0,2}$

$-\frac{1}{52} \sqrt{3} \sqrt{13} \sqrt{19} \phi_{0,2,2,2,0}-\frac{1}{156} \sqrt{3} \sqrt{13} \sqrt{5} \phi_{2,0,2,2,0}$

$\rho_{11}\left(\phi_{0,2,2,1,1}\right):=-\frac{1}{86} \sqrt{43} \sqrt{7} \phi_{0,2,1,1,2}$

$-\frac{1}{258} \sqrt{43} \sqrt{3} \sqrt{5} \sqrt{19} \phi_{2,0,1,1,2}+\frac{1}{12} \sqrt{43} \phi_{0,2,1,2,1}$

$-\frac{1}{1032} \sqrt{43} \sqrt{5} \sqrt{19} \sqrt{7} \phi_{1,1,1,2,1}-\frac{3}{344} \sqrt{43} \sqrt{19} \phi_{2,0,1,2 .}$. 
$+\frac{1}{12} \sqrt{43} \phi_{0,2,2,1,1}+\frac{1}{1032} \sqrt{43} \sqrt{5} \sqrt{19} \sqrt{7} \phi_{1,1,2,1,1}$

$-\frac{3}{344} \sqrt{43} \sqrt{19} \phi_{2,0,2,1,1}$

$\rho_{11}\left(\phi_{1,1,2,1,1}\right):=-\frac{1}{262} \sqrt{131} \sqrt{5} \sqrt{19} \phi_{0,2,1,1,2}$

$+\frac{7}{786} \sqrt{131} \sqrt{3} \sqrt{7} \phi_{2,0,1,1,2}+\frac{1}{1572} \sqrt{131} \sqrt{5} \sqrt{19} \sqrt{7} \phi_{0,2,1,2,1}$

$-\frac{1}{24} \sqrt{131} \phi_{1,1,1,2,1}-\frac{3}{1048} \sqrt{131} \sqrt{5} \sqrt{7} \phi_{2,0,1,2,1}$

$+\frac{1}{1572} \sqrt{131} \sqrt{5} \sqrt{19} \sqrt{7} \phi_{0,2,2,1,1}+\frac{1}{24} \sqrt{131} \phi_{1,1,2,1,1}$

$-\frac{3}{1048} \sqrt{131} \sqrt{5} \sqrt{7} \phi_{2,0,2,1,1}$

$\rho_{11}\left(\phi_{2,0,2,1,1}\right):=-\frac{1}{354} \sqrt{3} \sqrt{59} \sqrt{19} \sqrt{7} \phi_{0,2,1,1,2}$

$-\frac{1}{118} \sqrt{59} \sqrt{5} \phi_{2,0,1,1,2}-\frac{1}{236} \sqrt{3} \sqrt{59} \sqrt{19} \phi_{0,2,1,2,1}$

$+\frac{1}{472} \sqrt{3} \sqrt{59} \sqrt{5} \sqrt{7} \phi_{1,1,1,2,1}+\frac{1}{24} \sqrt{3} \sqrt{59} \phi_{2,0,1,2,1}$

$-\frac{1}{236} \sqrt{3} \sqrt{59} \sqrt{19} \phi_{0,2,2,1,1}-\frac{1}{472} \sqrt{3} \sqrt{59} \sqrt{5} \sqrt{7} \phi_{1,1,2,1,1}$

$+\frac{1}{24} \sqrt{3} \sqrt{59} \phi_{2,0,2,1,1}$

$\rho_{11}\left(\phi_{0,1,2,1,3}\right):=\frac{1}{3} \sqrt{3} \phi_{0,1,1,2,3}+\frac{1}{8} \sqrt{2} \phi_{0,1,1,3,2}$

$-\frac{1}{24} \sqrt{3} \sqrt{5} \sqrt{2} \phi_{1,0,1,3,2}+\frac{1}{3} \sqrt{3} \phi_{0,1,2,1,3}+\frac{1}{16} \sqrt{5} \phi_{0,1,2,3,1}$

$-\frac{7}{48} \sqrt{3} \phi_{1,0,2,3,1}+\frac{1}{8} \sqrt{2} \phi_{0,1,3,1,2}+\frac{1}{24} \sqrt{3} \sqrt{5} \sqrt{2} \phi_{1,0,3,1,2}$

$+\frac{1}{16} \sqrt{5} \phi_{0,1,3,2,1}+\frac{7}{48} \sqrt{3} \phi_{1,0,3,2,1}$

$\rho_{11}\left(\phi_{1,0,2,1,3}\right):=-\frac{1}{3} \sqrt{3} \phi_{1,0,1,2,3}+\frac{1}{24} \sqrt{3} \sqrt{2} \sqrt{5} \phi_{0,1,1,3,2}$

$+\frac{1}{8} \sqrt{2} \phi_{1,0,1,3,2}+\frac{1}{3} \sqrt{3} \phi_{1,0,2,1,3}-\frac{7}{48} \sqrt{3} \phi_{0,1,2,3,1}$

$-\frac{1}{16} \sqrt{5} \phi_{1,0,2,3,1}+\frac{1}{24} \sqrt{3} \sqrt{2} \sqrt{5} \phi_{0,1,3,1,2}-\frac{1}{8} \sqrt{2} \phi_{1,0,3,1,2}$

$-\frac{7}{48} \sqrt{3} \phi_{0,1,3,2,1}+\frac{1}{16} \sqrt{5} \phi_{1,0,3,2,1}$

$\rho_{11}\left(\phi_{0,2,2,2,0}\right):=-\frac{1}{32} \sqrt{3} \sqrt{2} \phi_{0,2,0,2,2}-\frac{1}{48} \sqrt{3} \sqrt{2} \sqrt{19} \phi_{1,1,0,2,2}$

$-\frac{1}{32} \sqrt{3} \sqrt{2} \sqrt{19} \phi_{2,0,0,2,2}-\frac{1}{32} \sqrt{3} \sqrt{2} \phi_{0,2,2,0,2}$

$+\frac{1}{48} \sqrt{3} \sqrt{2} \sqrt{19} \phi_{1,1,2,0,2}-\frac{1}{32} \sqrt{3} \sqrt{2} \sqrt{19} \phi_{2,0,2,0,2}$

$+\frac{1}{3} \sqrt{3} \sqrt{2} \phi_{0,2,2,2,0}$

$$
\rho_{11}\left(\phi_{1,1,2,2,0}\right):=0
$$

$\rho_{11}\left(\phi_{2,0,2,2,0}\right):=-\frac{7}{480} \sqrt{2} \sqrt{3} \sqrt{19} \sqrt{5} \phi_{0,2,0,2,2}$

$+\frac{3}{80} \sqrt{2} \sqrt{3} \sqrt{5} \phi_{1,1,0,2,2}-\frac{1}{96} \sqrt{2} \sqrt{3} \sqrt{5} \phi_{2,0,0,2,2}$

$-\frac{7}{480} \sqrt{2} \sqrt{3} \sqrt{19} \sqrt{5} \phi_{0,2,2,0,2}-\frac{3}{80} \sqrt{2} \sqrt{3} \sqrt{5} \phi_{1,1,2,0.2}$

$-\frac{1}{96} \sqrt{2} \sqrt{3} \sqrt{5} \phi_{2,0,2,0,2}+\frac{1}{3} \sqrt{2} \sqrt{3} \phi_{2,0,2,2,0}$

$\rho_{11}\left(\phi_{0,1,2,2,2}\right):=\phi_{0,1,2,2.2}$ 
$\rho_{11}\left(\phi_{1,0,2,2,2}\right):=0$

$\rho_{11}\left(\phi_{0,0,2.2,4}\right):=$

$\frac{1}{3} \sqrt{2} \sqrt{3} \phi_{0,0,2,2,4}-\frac{1}{6} \sqrt{2} \sqrt{3} \phi_{0,0,2,4,2}-\frac{1}{6} \sqrt{2} \sqrt{3} \phi_{0,0,4,2,2}$

$\rho_{11}\left(\phi_{0,1,2,3,1}\right):=\frac{1}{16} \sqrt{5} \phi_{0,1,1,2,3}+\frac{7}{48} \sqrt{3} \phi_{1,0,1,2,3}$

$+\frac{1}{48} \sqrt{3} \sqrt{2} \sqrt{5} \phi_{0,1,1,3,2}+\frac{3}{16} \sqrt{2} \phi_{1,0,1,3,2}+\frac{1}{16} \sqrt{5} \phi_{0,1,2,1,3}$ $-\frac{7}{48} \sqrt{3} \phi_{1,0,2,1,3}+\frac{1}{3} \sqrt{3} \phi_{0,1,2,3,1}+\frac{1}{48} \sqrt{3} \sqrt{2} \sqrt{5} \phi_{0,1,3,1,2}$

$-\frac{3}{16} \sqrt{2} \phi_{1,0,3,1,2}+\frac{1}{3} \sqrt{3} \phi_{0,1,3,2,1}$

$\rho_{11}\left(\phi_{1,0,2,3,1}\right):=-\frac{7}{48} \sqrt{3} \phi_{0,1,1,2,3}+\frac{1}{16} \sqrt{5} \phi_{1,0,1,2,3}$

$+\frac{3}{16} \sqrt{2} \phi_{0,1,1,3,2}-\frac{1}{48} \sqrt{3} \sqrt{5} \sqrt{2} \phi_{1,0,1,3,2}-\frac{7}{48} \sqrt{3} \phi_{0,1,2,1,3}$

$-\frac{1}{16} \sqrt{5} \phi_{1,0,2,1,3}+\frac{1}{3} \sqrt{3} \phi_{1,0,2,3,1}+\frac{3}{16} \sqrt{2} \phi_{0,1,3,1,2}$

$+\frac{1}{48} \sqrt{3} \sqrt{5} \sqrt{2} \phi_{1,0,3,1,2}-\frac{1}{3} \sqrt{3} \phi_{1,0,3,2,1}$

$\rho_{11}\left(\phi_{0,0,2,3,3}\right):=$

$\frac{1}{6} \sqrt{2} \sqrt{3} \phi_{0,0,2,3.3}+\frac{1}{6} \sqrt{2} \sqrt{3} \phi_{0,0,3,2.3}-\frac{1}{3} \sqrt{2} \sqrt{3} \phi_{0,0,3,3,2}$

$\rho_{11}\left(\phi_{0,0,2,4,2}\right):=$

$-\frac{1}{3} \sqrt{2} \sqrt{3} \phi_{0,0,2,2,4}+\frac{1}{6} \sqrt{2} \sqrt{3} \phi_{0,0,2,4,2}+\frac{1}{6} \sqrt{2} \sqrt{3} \phi_{0,0,4,2,2}$

$\rho_{11}\left(\phi_{0,1,3,0,3}\right):=\frac{1}{12} \sqrt{2} \sqrt{11} \sqrt{3} \phi_{0,1,0,3,3}$

$-\frac{1}{132} \sqrt{2} \sqrt{11} \sqrt{3} \sqrt{7} \phi_{1,0,0,3,3}+\frac{1}{12} \sqrt{2} \sqrt{11} \sqrt{3} \phi_{0,1,3,0,3}$

$+\frac{1}{132} \sqrt{2} \sqrt{11} \sqrt{3} \sqrt{7} \phi_{1,0,3,0,3}+\frac{1}{33} \sqrt{11} \sqrt{3} \phi_{0,1,3,3,0}$

$\rho_{11}\left(\phi_{1,0,3,0,3)}:=\frac{1}{60} \sqrt{2} \sqrt{5} \sqrt{3} \sqrt{7} \phi_{0,1,0,3,3}-\frac{1}{12} \sqrt{2} \sqrt{5} \sqrt{3} \phi_{1,0,0,3,3}\right.$

$+\frac{1}{60} \sqrt{2} \sqrt{5} \sqrt{3} \sqrt{7} \phi_{0,1,3,0,3}+\frac{1}{12} \sqrt{2} \sqrt{5} \sqrt{3} \phi_{1,0,3,0,3}$

$-\frac{1}{15} \sqrt{5} \sqrt{3} \sqrt{7} \phi_{0,1,3,3,0}$

$\rho_{11}\left(\phi_{0,1,3,1,2}\right):=\frac{1}{8} \sqrt{2} \phi_{0,1,1,2,3}-\frac{1}{24} \sqrt{3} \sqrt{2} \sqrt{5} \phi_{1,0,1,2,3}$

$+\frac{1}{3} \sqrt{3} \phi_{0,1,1,3,2}+\frac{1}{8} \sqrt{2} \phi_{0,1,2,1,3}+\frac{1}{24} \sqrt{3} \sqrt{2} \sqrt{5} \phi_{1,0,2,1,3}$

$+\frac{1}{48} \sqrt{3} \sqrt{5} \sqrt{2} \phi_{0,1,2,3,1}+\frac{3}{16} \sqrt{2} \phi_{1,0,2,3,1}+\frac{1}{3} \sqrt{3} \phi_{0,1,3,1,2}$

$+\frac{1}{48} \sqrt{3} \sqrt{5} \sqrt{2} \phi_{0,1,3,2,1}-\frac{3}{16} \sqrt{2} \phi_{1,0,3,2,1}$

$\rho_{11}\left(\phi_{1,0,3,1,2}\right):=\frac{1}{24} \sqrt{3} \sqrt{5} \sqrt{2} \phi_{0,1,1,2,3}+\frac{1}{8} \sqrt{2} \phi_{1,0.1,2,3}$

$-\frac{1}{3} \sqrt{3} \phi_{1,0.1,3,2}+\frac{1}{24} \sqrt{3} \sqrt{5} \sqrt{2} \phi_{0,1,2,1,3}-\frac{1}{8} \sqrt{2} \phi_{1,0,2,1,3}$

$-\frac{3}{16} \sqrt{2} \phi_{0,1,2,3,1}+\frac{1}{48} \sqrt{3} \sqrt{5} \sqrt{2} \phi_{1,0,2,3,1}+\frac{1}{3} \sqrt{3} \phi_{1,0,3,1.2}$

$-\frac{3}{16} \sqrt{2} \phi_{0,1,3,2.2}-\frac{1}{4 \bar{\gamma}} \sqrt{3} \sqrt{5} \sqrt{2} \phi_{1,0.3,2.2}$ 
$\rho_{11}\left(\phi_{0,0,3,1,4}\right):=\frac{1}{3} \sqrt{3} \phi_{0,0,1,3,4}-\frac{1}{6} \sqrt{3} \phi_{0,0,1,4,3}+\frac{1}{3} \sqrt{3} \phi_{0,0,3,1,4}$ $-\frac{1}{6} \sqrt{3} \phi_{0,0,3,4,1}-\frac{1}{6} \sqrt{3} \phi_{0,0,4,1,3}-\frac{1}{6} \sqrt{3} \phi_{0,0,4,3,1}$

$\rho_{11}\left(\phi_{0,1,3,2,1}\right):=\frac{1}{16} \sqrt{5} \phi_{0,1,1,2,3}+\frac{7}{48} \sqrt{3} \phi_{1,0,1,2,3}$ $+\frac{1}{48} \sqrt{3} \sqrt{2} \sqrt{5} \phi_{0,1,1,3,2}+\frac{3}{16} \sqrt{2} \phi_{1,0,1,3,2}+\frac{1}{16} \sqrt{5} \phi_{0,1,2,1,3}$ $-\frac{7}{48} \sqrt{3} \phi_{1,0,2,1,3}+\frac{1}{3} \sqrt{3} \phi_{0,1,2,3,1}+\frac{1}{48} \sqrt{3} \sqrt{2} \sqrt{5} \phi_{0,1,3,1,2}$ $-\frac{3}{16} \sqrt{2} \phi_{1,0,3,1,2}+\frac{1}{3} \sqrt{3} \phi_{0,1,3,2,1}$

$\rho_{11}\left(\varphi_{1,0,3,2,1}\right):=\frac{7}{48} \sqrt{3} \phi_{0,1,1,2,3}-\frac{1}{16} \sqrt{5} \phi_{1,0,1,2,3}-\frac{3}{16} \sqrt{2} \phi_{0,1,1,3,2}$

$+\frac{1}{48} \sqrt{3} \sqrt{5} \sqrt{2} \phi_{1,0,1,3,2}+\frac{7}{48} \sqrt{3} \phi_{0,1,2,1,3}+\frac{1}{16} \sqrt{5} \phi_{1,0,2,1,3}$ $-\frac{1}{3} \sqrt{3} \phi_{1,0,2,3,1}-\frac{3}{16} \sqrt{2} \phi_{0,1,3,1,2}-\frac{1}{48} \sqrt{3} \sqrt{5} \sqrt{2} \phi_{1,0,3,1,2}$ $+\frac{1}{3} \sqrt{3} \phi_{1,0,3,2,1}$

$\rho_{11}\left(\phi_{0,0,3,2,3)}:=\right.$ $\frac{1}{6} \sqrt{2} \sqrt{3} \phi_{0,0,2,3,3}+\frac{1}{6} \sqrt{2} \sqrt{3} \phi_{0,0,3,2,3}-\frac{1}{3} \sqrt{2} \sqrt{3} \phi_{0,0,3,3,2}$ $\rho_{11}\left(\phi_{0,1,3,3,0}\right):=\frac{1}{12} \sqrt{3} \phi_{0,1,0,3,3}+\frac{1}{12} \sqrt{3} \sqrt{7} \phi_{1,0,0,3,3}$ $+\frac{1}{12} \sqrt{3} \phi_{0,1,3,0,3}-\frac{1}{12} \sqrt{3} \sqrt{7} \phi_{1,0,3,0,3}+\frac{1}{3} \sqrt{2} \sqrt{3} \phi_{0,1,3,3,0}$ $\rho_{11}\left(\phi_{1,0,3,3,0}\right):=0$

$\rho_{11}\left(\phi_{0,0,3,3,2}\right):=$ $-\frac{1}{6} \sqrt{2} \sqrt{3} \phi_{0,0,2,3,3}-\frac{1}{6} \sqrt{2} \sqrt{3} \phi_{0,0,3,2,3}+\frac{1}{3} \sqrt{2} \sqrt{3} \phi_{0,0,3,3,2}$ $\rho_{11}\left(\phi_{0,0,3,4,1}\right):=-\frac{1}{6} \sqrt{3} \phi_{0,0,1,3,4}-\frac{1}{6} \sqrt{3} \phi_{0,0,1,4,3}-\frac{1}{6} \sqrt{3} \phi_{0,0,3,1,4}$ $+\frac{1}{3} \sqrt{3} \phi_{0,0,3,4,1}-\frac{1}{6} \sqrt{3} \phi_{0,0,4,1,3}+\frac{1}{3} \sqrt{3} \phi_{0,0,4,3,1}$

$\rho_{11}\left(\phi_{0,0,4,0,4}\right):=$ $\frac{1}{6} \sqrt{2} \sqrt{3} \phi_{0,0,0,4,4}+\frac{1}{6} \sqrt{2} \sqrt{3} \phi_{0,0,4,0,4}-\frac{1}{3} \sqrt{2} \sqrt{3} \phi_{0,0,4,4,0}$ $\rho_{11}\left(\phi_{0,0,4,1,3}\right):=-\frac{1}{6} \sqrt{3} \phi_{0,0,1,3,4}+\frac{1}{3} \sqrt{3} \phi_{0,0,1,4,3}-\frac{1}{6} \sqrt{3} \phi_{0,0,3,1,4}$ $-\frac{1}{6} \sqrt{3} \phi_{0,0,3,4,1}+\frac{1}{3} \sqrt{3} \phi_{0,0,4,1,3}-\frac{1}{6} \sqrt{3} \phi_{0,0,4,3,1}$

$\rho_{11}\left(\phi_{0,0,4,2,2}\right):=$ $-\frac{1}{3} \sqrt{2} \sqrt{3} \phi_{0,0,2,2,4}+\frac{1}{6} \sqrt{2} \sqrt{3} \phi_{0,0,2,4,2}+\frac{1}{6} \sqrt{2} \sqrt{3} \phi_{0,0,4,2,2}$ $\rho_{11}\left(\varphi_{0,0,4,3,1}\right):=-\frac{1}{6} \sqrt{3} \phi_{0,0,1,3,4}-\frac{1}{6} \sqrt{3} \phi_{0,0,1,4,3}-\frac{1}{6} \sqrt{3} \phi_{0,0,3,1,4}$ $+\frac{1}{3} \sqrt{3} \phi_{0,0,3,4,1}-\frac{1}{6} \sqrt{3} \phi_{0,0,4,1,3}+\frac{1}{3} \sqrt{3} \phi_{0,0,4,3,1}$

$\rho_{11}\left(\phi_{0,0,4.4 .0}\right):=$ 
$-\frac{1}{6} \sqrt{2} \sqrt{3} \phi_{0,0,0,4,4}-\frac{1}{6} \sqrt{2} \sqrt{3} \phi_{0,0,4,0,4}+\frac{1}{3} \sqrt{2} \sqrt{3} \phi_{0,0,4,4,0}$
NumFrncoes $:=70$

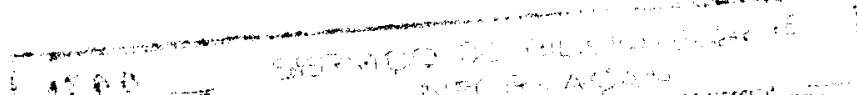

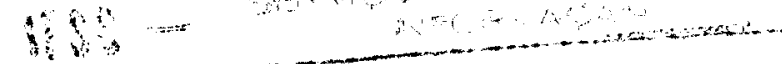




\section{Apêndice C}

\section{Bases para as Funções de Canal,}

\section{Resultantes do Projetor $\rho_{11}^{(m)}$}

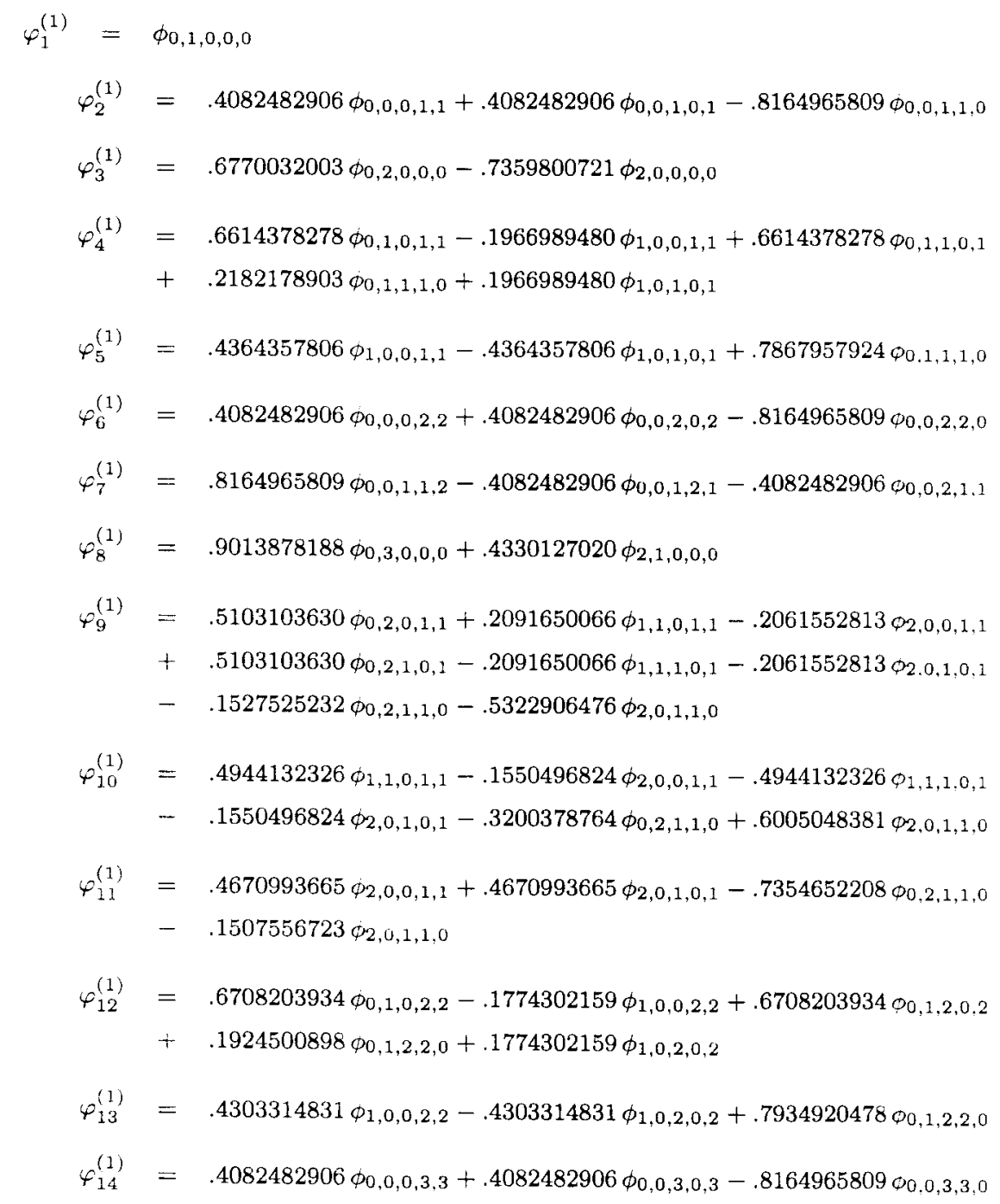




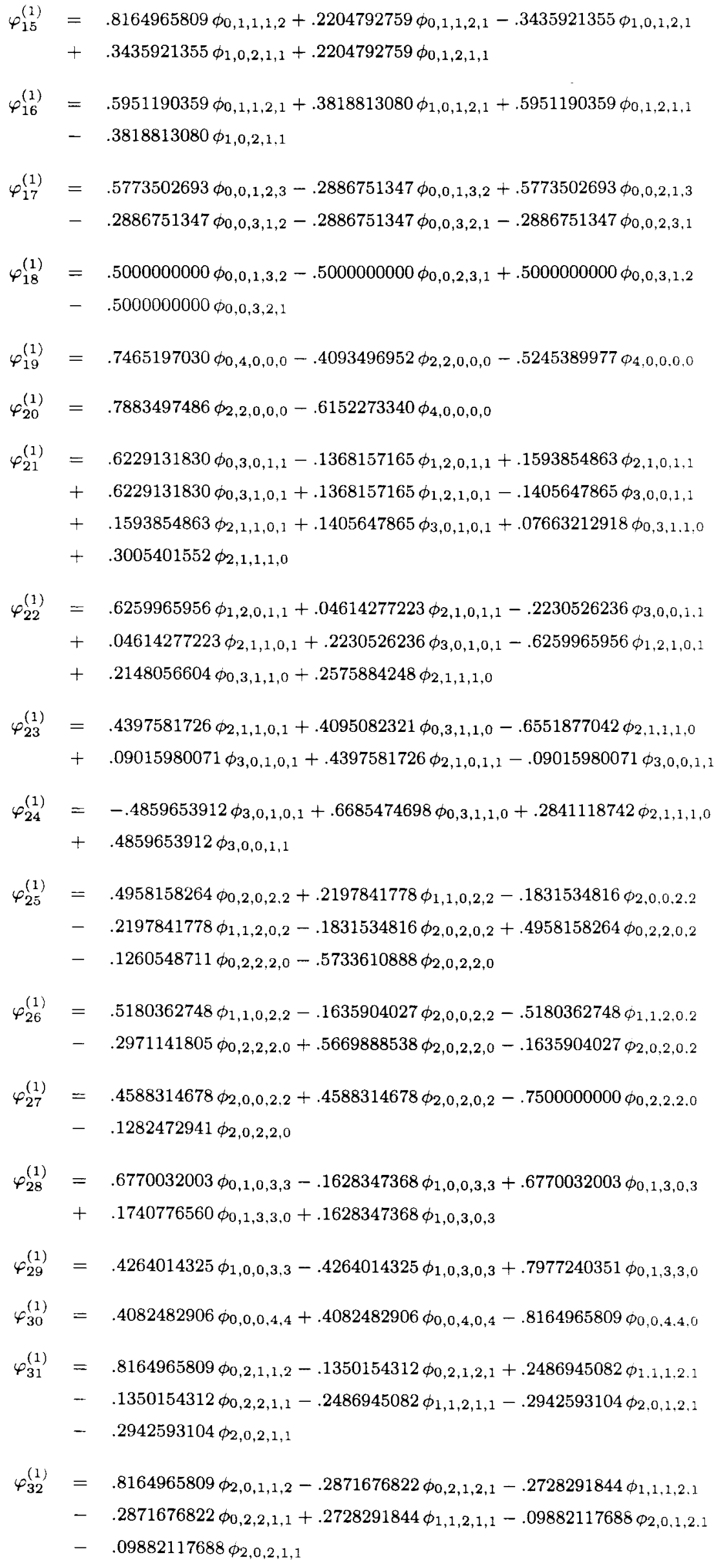




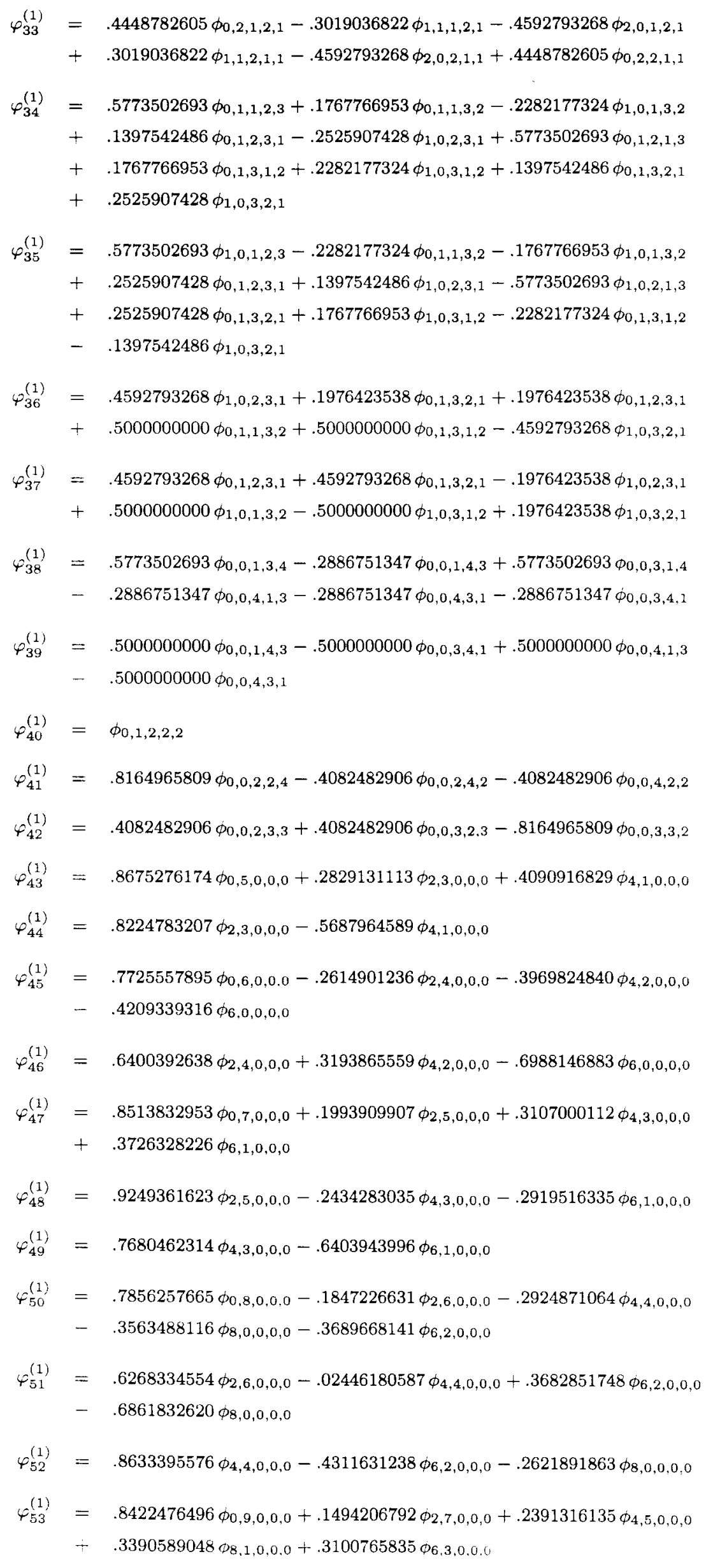




$$
\begin{aligned}
& \varphi_{54}^{(1)}=.9386235230 \phi_{2,7,0,0,0}+.01469452991 \phi_{4,5,0,0,0}-.3177609701 \phi_{6,3,0,0,0} \\
& -.1334088395 \phi_{8,1,0,0,0} \\
& \varphi_{55}^{(1)}=.5996092849 \phi_{4,5,0,0,0}+.3332142304 \phi_{6,3,0,0,0}-.7276242035 \phi_{8,1,0,0,0} \\
& \varphi_{56}^{(1)}=.7932789768 \phi_{0,10,0,0,0}-.1392380851 \phi_{2,8,0,0,0}-.2245240726 \phi_{4,6,0,0,0} \\
& \text { - } .3409219607 \phi_{8,2,0,0,0}-.3113823691 \phi_{10,0,0,0,0}-.2961813621 \phi_{6,4,0,0,0} \\
& \varphi_{57}^{(1)}=.6481258774 \phi_{2,8,0,0,0}-.2142585444 \phi_{4,6,0,0,0}+.1960636653 \phi_{6,4,0,0,0} \\
& \text { - } .6408434303 \phi_{10,0,0,0,0}+.2913843881 \phi_{8,2,0,0,0} \\
& \varphi_{58}^{(1)}=.9013716751 \phi_{4,6,0,0,0}-.1250819030 \phi_{6,4,0,0,0}-.1234755561 \phi_{8,2,0,0,0} \\
& \text { - } .3957744390 \phi_{10,0,0,0,0} \\
& \varphi_{59}^{(1)}=.7756502620 \phi_{6,4,0,0,0}-.6292723463 \phi_{8,2,0,0,0}-.04881583051 \phi_{10,0,0,0,0} \\
& \varphi_{60}^{(1)}=.8364982265 \phi_{0,11,0,0,0}+.1170810023 \phi_{2,9,0,0,0}+.1898482982 \phi_{4,7,0,0,0} \\
& +.2999891704 \phi_{8,3,0,0,0}+.3103682470 \phi_{10,1,0,0,0}+.2533740867 \phi_{6,5,0,0,0} \\
& \varphi_{61}^{(1)}=.9316482141 \phi_{2,9,0,0,0}+.1307617914 \phi_{4,7,0,0,0}-.1255417799 \phi_{6,5,0,0,0} \\
& \text { - } .02556489016 \phi_{10,1,0,0,0}-.3138768179 \phi_{8,3,0,0,0} \\
& \varphi_{62}^{(1)}=.6471610739 \phi_{4,7,0,0,0}-.1585299590 \phi_{6,5,0,0,0}+.3850290185 \phi_{8,3,0,0,0} \\
& \text { - } .6385949043 \phi_{10,1,0,0,0} \\
& \varphi_{63}^{(1)}=.8610954994 \phi_{6,5,0,0,0}-.3116959455 \phi_{8,3,0,0,0}-.4016966259 \phi_{10,1,0,0,0} \\
& \varphi_{64}^{(1)}=.7982145527 \phi_{0,12,0,0,0}-.1097503858 \phi_{2,10,0,0,0}-.1787161430 \phi_{4,8,0,0,0} \\
& \text { - } .2898709878 \phi_{8,4,0,0,0}-.3158295086 \phi_{10,2,0,0,0}-.2405183277 \phi_{6,6,0,0,0} \\
& -\quad .2779323404 \phi_{12,0,0,0,0} \\
& \varphi_{65}^{(1)}=.6727846755 \phi_{2,10,0,0,0}-.2754690232 \phi_{4,8,0,0,0}-.00951005366 \phi_{6,6,0,0,0} \\
& +.1962149881 \phi_{10,2,0,0,0}-.5953623184 \phi_{12,0,0,0,0}+.2800545370 \phi_{8,4,0,0,0} \\
& \varphi_{66}^{(1)}=.8335627723 \phi_{4,8,0,0,0}+.1440018067 \phi_{6,6,0,0,0}-.2289013258 \phi_{8,4,0,0,0} \\
& \text { - } .4790699154 \phi_{12,0,0,0,0}+.05032676952 \phi_{10,2,0,0,0} \\
& \varphi_{67}^{(1)}=-.7181575231 \phi_{10,2,0,0,0}-.07386128533 \phi_{12,0,0,0,0}+.5871777425 \phi_{6,6,0,0,0} \\
& +.3660827522 \phi_{8,4,0,0,0} \\
& \varphi_{68}^{(1)}=.8326072554 \phi_{0,13,0,0,0}+.09483305244 \phi_{2,11,0,0,0}+.1549356536 \phi_{4,9,0,0,0} \\
& +.2559632636 \phi_{8,5,0,0,0}+.2869675026 \phi_{10,3,0,0,0}+.2098195264 \phi_{6,7,0,0,0} \\
& +.2861381151 \phi_{12,1,0,0,0} \\
& \varphi_{69}^{(1)}=.9201997413 \phi_{2,11,0,0,0}+.1769049817 \phi_{4,9,0,0,0}+.01351069325 \phi_{6,7,0,0,0} \\
& \text { - } .2775450738 \phi_{10,3,0,0,0}+.05122143285 \phi_{12,1,0,0,0}-.2051819211 \phi_{8,5,0,0,0} \\
& \varphi_{70}^{(1)}=.7295489617 \phi_{4,9,0,0,0}-.3286721533 \phi_{6,7,0,0,0}+.1281625882 \phi_{8,5,0,0,0} \\
& -\quad .5265073581 \phi_{12,1,0,0,0}+.2570939155 \phi_{10,3,0,0,0} \\
& \varphi_{71}^{(1)}=-.00484668466 \phi_{10,3,0,0,0}-.5426439646 \phi_{12,1,0,0,0}+.8366991260 \phi_{6,7,0,0,0} \\
& -\quad .07381470376 \phi_{8,5,0,0,0} \\
& \varphi_{72}^{(1)}=-.1021814187 \phi_{12,1,0,0,0}-.6034791838 \phi_{10,3,0,0,0}+.7908045471 \phi_{8,5,0,0,0}
\end{aligned}
$$


$\varphi_{73}^{(1)}=.8016166533 \phi_{0,14,0,0,0}-.08937030504 \phi_{2,12,0,0,0}-.1463954122 \phi_{4,10,0,0,0}$

- $.2451633960 \phi_{8,6,0,0,0}-.2798754197 \phi_{10,4,0,0,0}-.1992093901 \phi_{6,8,0,0,0}$

- $.2939636615 \phi_{12,2,0,0,0}-.2519081511 \phi_{14,0,0,0,0}$

$\varphi_{74}^{(1)}=.6941669569 \phi_{2,12,0,0,0}-.2820098580 \phi_{4,10,0,0,0}-.1287392930 \phi_{6,8,0,0,0}$

$+.2922975798 \phi_{10,4,0,0,0}+.1117276487 \phi_{12,2,0,0,0}+.1233740388 \phi_{8,6,0,0,0}$

$-\quad .5557758313 \phi_{14,0,0,0,0}$

$\varphi_{75}^{(1)}=.7713709424 \phi_{4,10,0,0,0}+.1835041110 \phi_{6,8,0,0,0}+.02134140705 \phi_{8,6,0,0,0}$

$+.1669338544 \phi_{12,2,0,0,0}-.5284202606 \phi_{14,0,0,0,0}-.2525129120 \phi_{10,4,0,0,0}$

$\varphi_{76}^{(1)}=-.5467681241 \phi_{12,2,0,0,0}+.7000809025 \phi_{6,8,0,0,0}-.2246551315 \phi_{8,6,0,0,0}$

- $.1211441010 \phi_{14,0,0,0,0}+.3818187094 \phi_{10,4,0,0,0}$

$\varphi_{7 \tau}^{(1)}=.8464314442 \phi_{8,6,0,0,0}-.4892865311 \phi_{12,2,0,0,0}-.02045172176 \phi_{14,0,0,0,0}$

- $.2091273036 \phi_{10,4,0,0,0}$ 


\section{Bibliografia}

[1] M. Born and V. Fock, Zs. f. Phys. 51, 165 (1928)

[2] T. Kato, Journ. Phys. Soc. Jap. 5, 435 (1950)

[3] Albert Messiah, Quantum Mechanics, vol. II; 3rd Edition, Amsterdam: North Holland Publishing Company, (1965).

[4] M. Born and R. Oppenheimer, Ann. Phys. 84, 475 (1927)

[5] Asckroft and Mermin, Solid State Physics, Saunders College Publishing, International Edition, (1976)

[6] V. Fock, Z. Physik 61, 126 (1930)

[7] Macek, J. H., J. Phys. B 1, 831 (1968)

[8] Smirnov Yu, F. and Shitikova, V. K., Sov. J. Part. Nucl. 8, n.4, $3444^{*}$

[9] Coelho, H. T. and Hornos, J. E., Phys. Rev A 43. 6379 (1991)

[10] Fano, U., Rep. Prog. Phys. 46, 97 (1983)

[11] Greene, C. H., Phys. Rev. A 23, 661 (1981); Greene, C. H., Phys. Rev. A 22, 149 (1986)

[12] Lin, C. D., Phys. Rev. A 12, 493 (1975); Lin, C. D.. Phys. Rev. A 14, 14 (1976)

[13] Hornos, J. E., MacDowell, S. W. and Caldwell, C. D.. Phys. Rev. A 33. 2212 (1986)

[14] De Groote, J. J., Hornos, J. E. and Coelho, H. T. Phys. Rer. B 46. 2101 (1992)

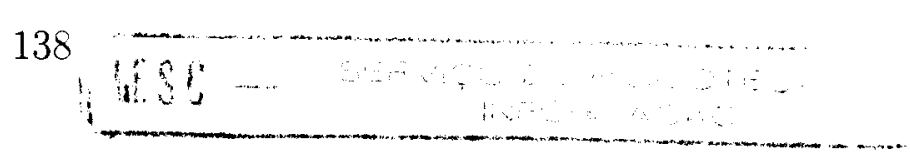


[15] Coelho, H. T., De Groote, J. J. and Hornos, Phys. Rev. A 46, n.9, 5443 (1992)

[16] Masili, M., Hornos, J. E. and De Groote, J. J., Phys. Rev. A 52, 3362 (1995)

[17] Lin, C. D., Phys. Rep. 257, 1 (1995)

[18] Greene, C. H. and Clark, C. W., Phys. Rev. A 30, 2161 (1984)

[19] Clark, C. W. and Greene, C. H., Phys. Rev. A 21, 1786 (1980)

[20] Xiazhou Yang, Bao, C. G. and Lin, C. D., Phys. Rev. A 53, 3934 (1996)

[21] Xiazhou Yang, Jinhua Xi, Bao, C. G. and Lin, C. D., Phys. Rev. A 52, 2029 (1995)

[22] Bao, C. G. and Lin, C. D., Few-Body System 16, 47 (1994)

[23] Yixuan Wang, Conghao Deng and Dacheng Feng, Phys. Rev. A 51, 73 (1995)

[24] Lauro Tomio, Cálculo de Energia do Estado Fundamental do Átomo de Lítio no Formalismo Hiperesférico, Dissertação de Mestrado (1974), IFT

[25] Mauro Masili, Acoplamentos Não-Adiabáticos pelo Método Hiperesférico, Dissertação de Mestrado (1993), USP/IFSC

[26] Milton Abramowitz and Irene A. Stegun, Handbook of Mathematical Functions, 3rd Edition, Washington: (March 1965).

[27] Cohen-Tannoudji, C.; Quantum Mechanics, vol II, por Hermann Company, Paris (1973).

[28] Jin-Quan Chen; Group Theory for Physicists, World Scientific Publishing Co. Pte. Ltd. .

[29] Hamermesh, M.; Group Theory and its Application to Physical Problems, 2nd Edition, Addison-Wesley Publishing Company INC.

[30] O. Jitrik and C.F. Bunge, Phys. Rev. A 43, 5809 (1991)

[31] J. Q. Sun and C. D. Lin, J. Phys. B 25, 1363 (1992)

[32] Zheng Zhen and Joseph Macek, Phys. Rev. A 41. 4696 (1990) 